\title{
The effect of roller compaction and tableting stresses on pharmaceutical tablet performance
}

\section{Arthi Devi Rajkumar}

Chemical and Biological Engineering Department The University of Sheffield

January 2018

A thesis submitted in partial fulfilment of the requirements for the degree of Doctor of Philosophy

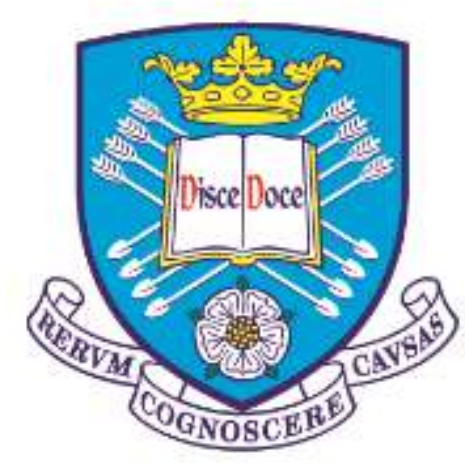




\section{Abstract}

For tablets made by roller compaction the relationships between formulation composition, manufacturing conditions and in-vitro performance were examined. Two filler materials with different characteristics were studied. During tablet production, two main stresses are experienced by a material. The first stress occurs during granulation and the second stress during tableting. These stresses were investigated in terms of the tablet properties and disintegration behaviour. Roller compaction granulation, a continuous dry granulation process, was used to produce granules. The granules were then compacted using varying tableting stresses.

The tablets were characterised by their porosity and tensile strength (i.e. compactibility), and were also examined using X-ray to determine the internal tablet structure. The tablet internal structure indicated that the final tablet compactibility was dependent on the RC stress used, which governs the consequent granular properties. The granular material is then affected by the tableting stress used depending on the granular properties and the magnitude of the stress. The tableting stress was found to crush and bond the granular material differently, depending on the how compacted the granular material is.

Two different particle imaging methods, as well as the standard USP method, were employed to determine which method was most capable of distinguishing the effects of processing condition variation on tablet disintegration performance. The methods were evaluated to determine their effectiveness at monitoring disintegration and the information which is able to be gained from them. By using imaging methods to examine tablet disintegration, quantifiable information regarding the processes which occur during disintegration was observed, including the particle release and change in tablet area with time. It was found that the tableting stress has a greater influence on the tablet disintegration than roller compaction stress.

The findings in this research add to a better understanding of tablet behaviour during disintegration due to variation in the formulation and processing conditions. This could lead to more knowledge-based approach to the innovation of future tablet formulations. 


\section{Acknowledgments}

First and foremost I would like to show my gratitude to my supervisor Prof Agba Salman for being such a great mentor and guide through the whole of my PhD journey. You have provided unwavering support and I will never be able to thank you enough for everything you have done for me.

Secondly, I want to thank AstraZeneca for their funding of the project. I want to especially thank my industrial supervisors Prof Gavin Reynolds, Dr David Wilson and Dr Stephen Wren for all of the support and direction you have given me during my $\mathrm{PhD}$. Your expertise has been invaluable to me and my research, and working with you has been an honour.

I would also like to show my huge appreciation for my group members who became like family to me. Thank you to my seniors Chalak, Mohammed, Riyadh, William and Syed for all of your help, and for keeping PhD life jovial even when times were tough. Thank you to Jiankai and Qing for the Indo-Chinese meetings and for introducing me to authentic Chinese food. Thank you to Claire for all of the good times we shared and most importantly for being my friend. And big thanks to all of the old and new members in the big PPG family and GRAND members throughout the years for all of the encouragement and support; it was a really great experience to know you all. Thank you to all of my friends for sticking by me throughout this journey.

Thank you so much to Sushma, my sister in the group. When I joined the group I never expected that I would find such a close friend in someone. I am proud to be part of the Indian Society with you, Ranjit and honorary member Menan; I look forward to many more meetings in the future.

My appreciation goes out to my mum, my dad and my sister. Every day I worked hard because of you. Thank you for the motivation and the unconditional love whenever I would need it. I hope I can make you proud.

Last but not least, thank you to Daniel. Thank you for having faith in me even when I didn't. You are an inspiration to me and without you none of this would have been possible, I love you. 


\section{Publications}

\section{Journal papers:}

- The effect of roller compaction and tableting stresses on pharmaceutical tablet performance, A. D. Rajkumar, G. K. Reynolds, D. Wilson, S. Wren \& A. D. Salman. Submitted to Powder Technology, Nov 2017.

- Investigating the effect of processing parameters on pharmaceutical tablet disintegration using a real-time particle imaging approach, A. D. Rajkumar, G. K. Reynolds, D. Wilson, S. Wren, M. J. Hounslow \& A. D. Salman. European Journal of Pharmaceutics and Biopharmaceutics, 2016, 106, p88-96.

\section{Conference papers:}

- "The effect of roller compaction and tableting stresses on pharmaceutical tablet performance", oral presentation, poster presentation and conference paper no. 20 at the 8th International Granulation Workshop, Sheffield, United Kingdom, June 2017.

- "Real-time particle imaging of pharmaceutical tablet disintegration", poster presentation and conference paper no. 94 at the 7th International Granulation Workshop, Sheffield, United Kingdom, July 2015. 


\section{Table of Contents}

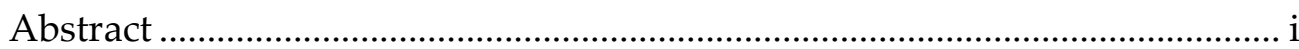

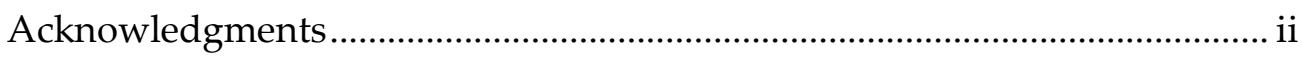

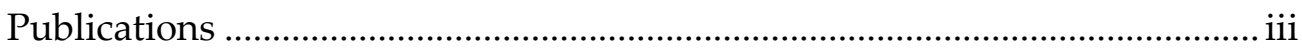

Table of Contents ........................................................................................... iv

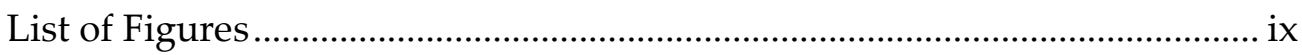

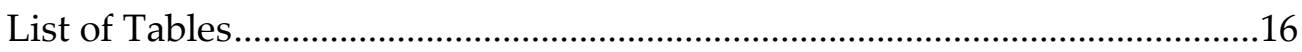

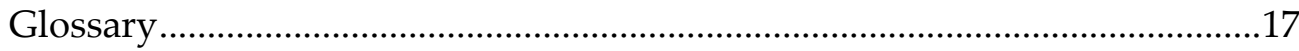

Chapter 1. Introduction ................................................................ 19

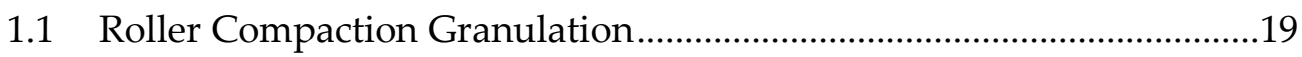

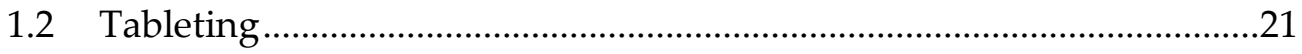

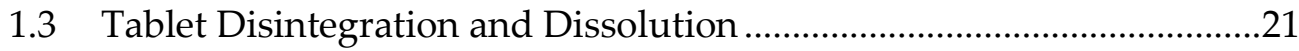

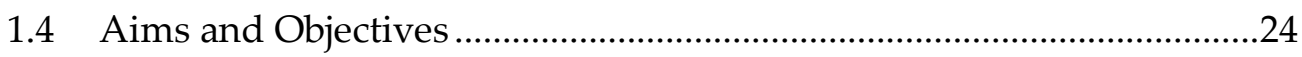

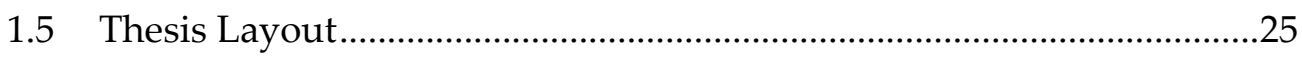

Chapter 2. Literature Review ................................................. 27

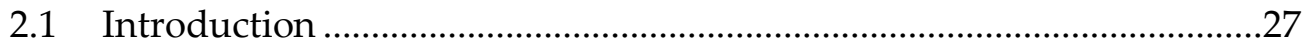

2.2 Factors Affecting Disintegration and Dissolution .................................28

2.2.1 Pharmaceutical Tablet Formulation .....................................................28

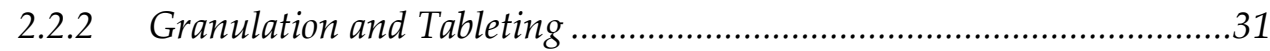

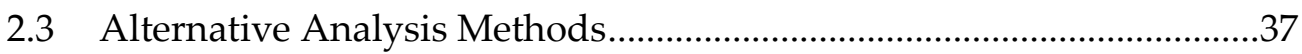




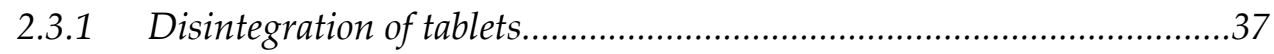

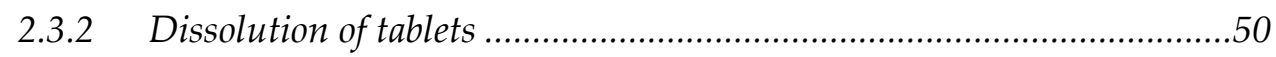

\section{Chapter 3. Roller Compaction and Tableting........................ 59}

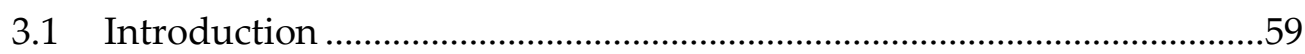

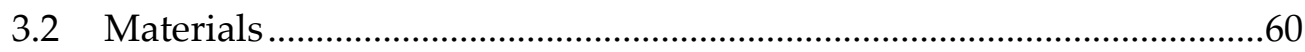

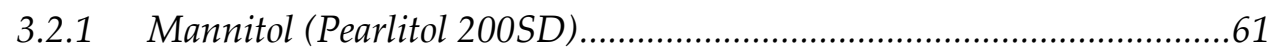

3.2.2 Microcrystalline cellulose (Avicel PH-101).......................................61

3.2.3 Magnesium stearate (Mallinkrodt)................................................62

3.2.4 Filler particle size and solubility.....................................................62

3.2.5 Binary placebo mixtures .................................................................63

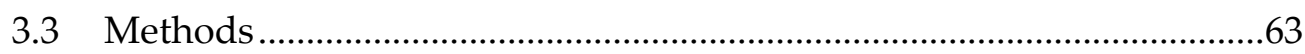

3.3.1 Scanning electron microscopy (SEM) ............................................63

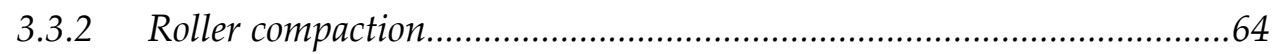

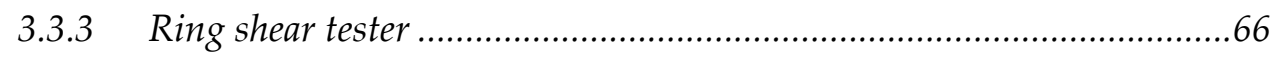

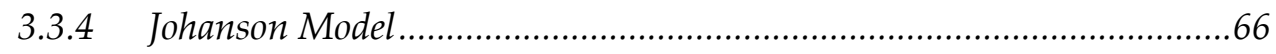

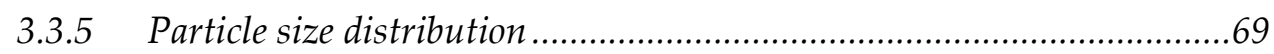

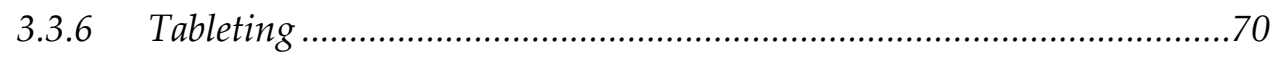

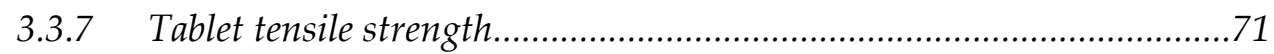

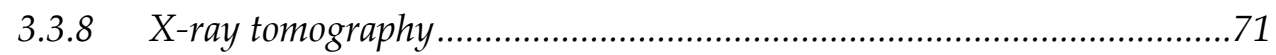

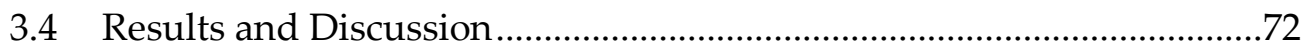

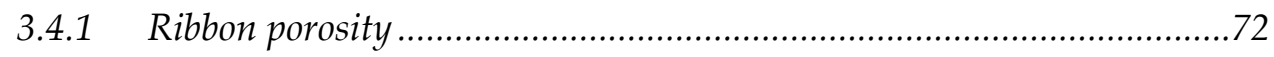


3.4.2 Granule size distribution .73

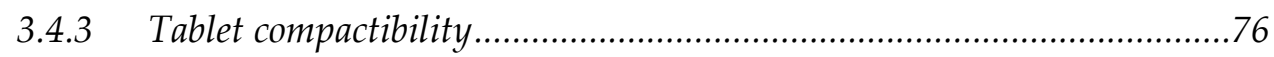

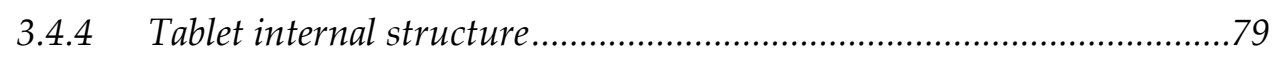

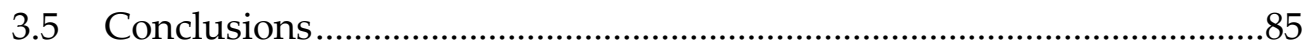

Chapter 4. LIXELL Particle Imaging and ${ }^{1} \mathrm{H}$ NMR ........... 87

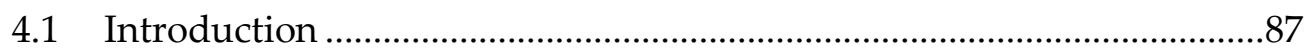

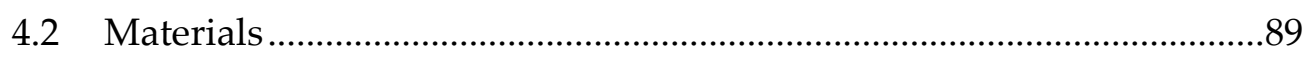

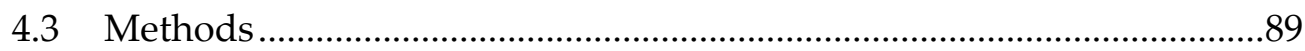

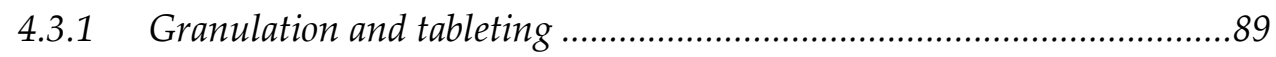

4.3.2 LIXELL particle imaging disintegration analysis ...............................90

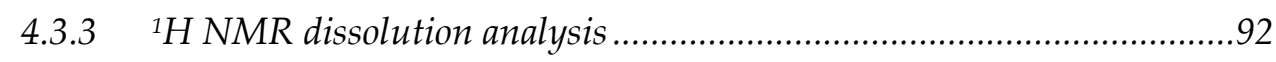

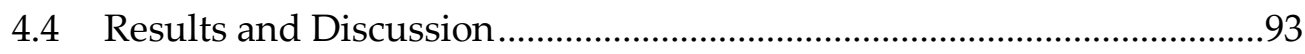

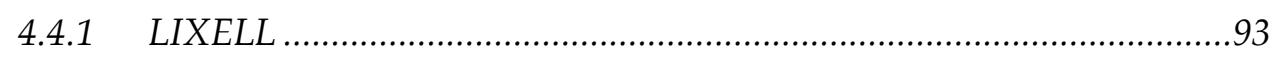

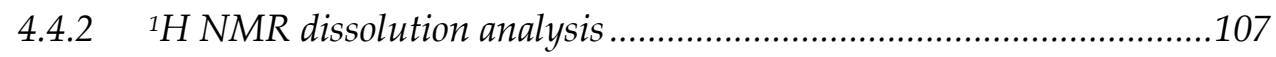

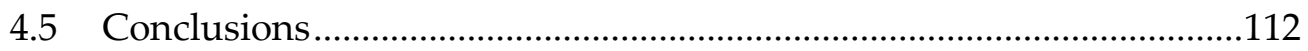

Chapter 5. Flow Cell Imaging................................................. 114

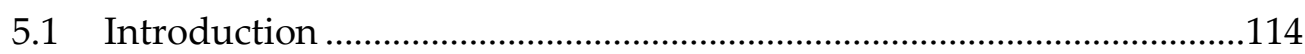

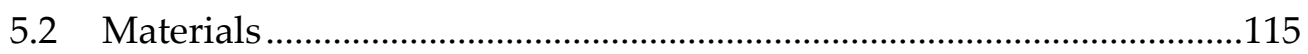

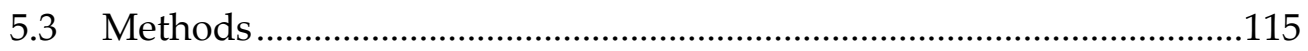

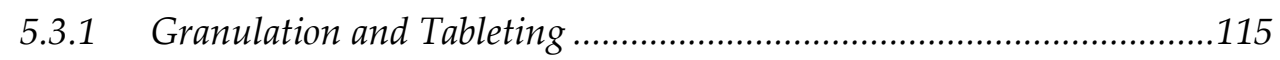

5.3.2 Flow Cell disintegration analysis....................................................115 
5.4 Results and Discussion

5.4.1 Imaging.....

5.4.2 Tablet area and particle release.....

5.5 Conclusions

Chapter 6. USP vs Flow Cell Imaging.................................. 136

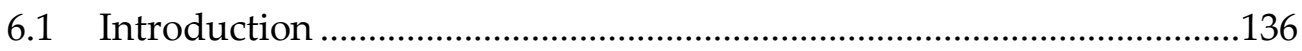

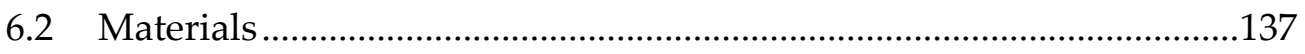

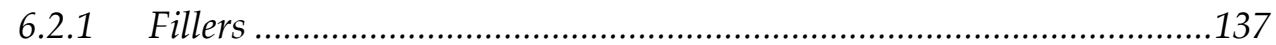

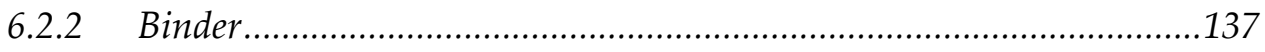

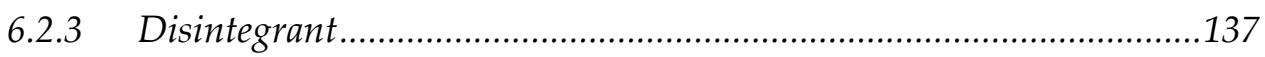

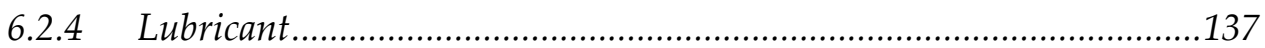

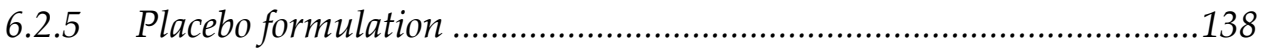

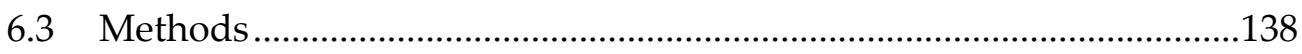

6.3.1 Granulation and tableting .........................................................138

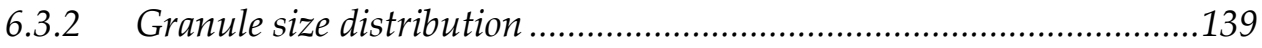

6.3.3 Tablet tensile strength and porosity ................................................139

6.3.4 USP disintegration analysis ......................................................140

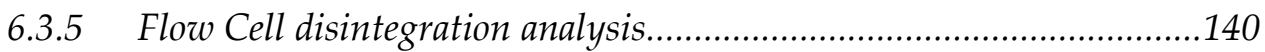

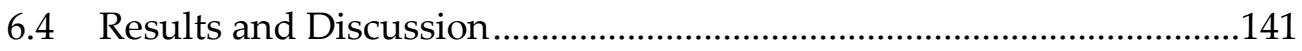

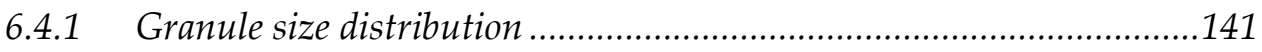

6.4.2 Tablet tensile strength and porosity ................................................142 


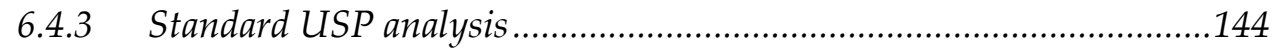

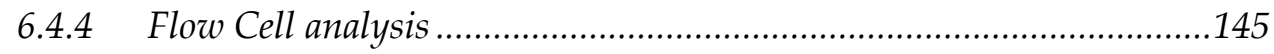

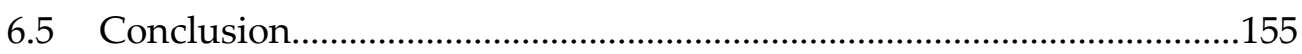

Chapter 7. Conclusions and Future Work ........................... 157

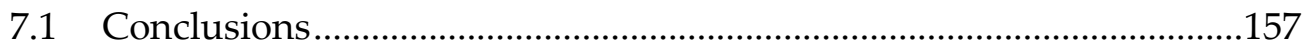

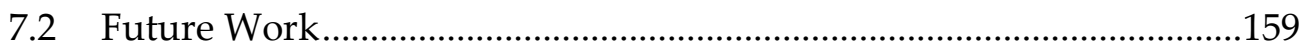

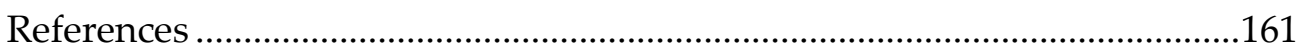

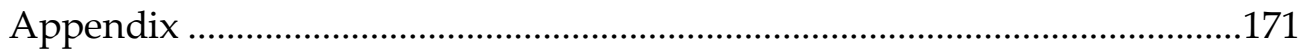




\section{List of Figures}

Figure 1.1 Schematic of tablet disintegration showing the breakage of agglomerates released from a tablet.

Figure 1.2 Schematic of tablet dissolution showing the particles from a tablet dissolving into solution

Figure 1.3 Image of a USP II apparatus with 8 vessels. 23

Figure 2.1 Tablet tensile strength for a formulation containing $72 \%$ Avicel PH102, $24 \%$ lactose monohydrate, $3 \%$ croscarmellose sodium and $1 \%$ magnesium stearate. Tablets were produced using various compaction pressures using granulated material (40 or 80 bar RC pressure), or directly compressed from the powder (Farber et al., 2008). .33

Figure 2.2 Tablet compactibility graphs of sieved MCC granules produced by different numbers of roller compactions, highlighting that larger granules exhibit lower compactibility (Sun and Himmelspach, 2006)....

Figure 2.3 Tabletability plot of the three sieved fractions of mannitol granules. Each point is one tablet. (Wu and Sun, 2007).

Figure 2.4 Illustration of Flow Cell and a) typical image obtained during disintegration testing using the Flow Cell. b) Converted binary image after thresholding (white - tablet and particles, and black - background) (Mesnier et al., 2013).

Figure 2.5 Dissolution profiles of tablets made of 100\% maltodextrin DE 21 and maltodextrin DE 21 containing $2 \%$ of disintegrant (croscarmellose sodium). (Mesnier et al., 2013).

Figure 2.6 a) Variation of tablet thickness with time for tablets made of $100 \%$ maltodextrin DE 21 and maltodextrin DE 21 containing 2\% of disintegrant. b) Variation of tablet diameter with time for tablets made of $100 \%$ maltodextrin 
DE 21 and maltodextrin DE 21 containing 2\% of disintegrant. (Mesnier et al., 2013) 42

Figure 2.7 Density distribution of the particles liberated during the disintegration of tablets of maltodextrin DE21 and tablets of maltodextrin DE21 containing $2 \%$ of disintegrants (croscarmellose sodium) obtained for the first 150s. (Mesnier et al., 2013) 43

Figure 2.8 Variation of the number of the particles liberated during the disintegration of tablets of maltodextrin DE21 containing $2 \%$ of disintegrant (croscarmellose sodium) plotted for two different times: particles liberated from the start to 90 s, and particle liberated after 90s to 150s. (Mesnier et al., 2013)

Figure 2.9 Dissolution profile obtained using UV analysis for Tablet A ( $\mathbf{\square})$ and Tablet B ( $\mathbf{\Delta})$. (Wilson et al., 2012)

Figure 2.10 Number of particles released with time for Tablet A ( $\mathbf{\square})$ and Tablet B ( $\mathbf{A})$. (Wilson et al., 2012) 48

Figure 2.11 Mass weighted particle size distributions of tablet A (top images) and tablet B (bottom image). Left side images show the total distribution; right side images show three small size classes in the approximate location of the drug substance. (Wilson et al., 2012)...

Figure 2.12 Various proposed routes for dissolution to occur. (Ibrahim and Sallam, 1993) 51

Figure 2.13 Evolution of a) $\mathrm{M}_{\mathrm{s}}$ b) $\mathrm{V}$ and c) $\mathrm{M}_{\mathrm{w}}$ with time for theophylline tablets produced using varying tableting forces. (Delalonde and Ruiz, 2008)53

Figure 2.14 A) water penetration profiles for La-H ( $\Delta$ ), La-M ( $\square)$, La-L (०) and Tr-M (匹) vs time and B) W2 vs time. Solid lines are fit to linear lines on the basis of the Lucas-Washburn theory. (Hattori and Otsuka, 2011) 56

Figure 3.1 SEM images of Mannitol Pearlitol 200SD at x50 and x250 magnification. 61 
Figure 3.2 SEM images of MCC Avicel PH101 at x50 and x250 magnification.62

Figure 3.3 Photograph and schematic of the roller compactor used in this research. 65

Figure 3.4 Schematic diagram showing the roller compaction process. 67

Figure 3.5 Diagram showing compression of granules into a tablet. 70

Figure 3.6 Ribbon porosities calculated for Man100:MCC0, Man75:MCC25, Man50:MCC50, Man25:MCC75 and Man0:MCC100 ribbons which were produced using 20 or 100 bar roller compaction pressure. 72

Figure 3.7 Granule size distribution data for mannitol granules produced using roller compaction at various pressures. 74

Figure 3.8 Granule size distribution data for MCC granules produced using roller compaction at various pressures.

Figure $3.9 \mathrm{D}_{10}, \mathrm{D}_{50}$ and $\mathrm{D}_{90}$ values for mannitol granules produced using roller compaction at various pressures. 75

Figure $3.10 \mathrm{D}_{10}, \mathrm{D}_{50}$ and $\mathrm{D}_{90}$ values for MCC granules produced using roller compaction at various pressures 75

Figure 3.11 Compactibility graph for tablets produced using varying ratios of mannitol and MCC, using either 20 or 100 bar RC pressures and using $5 \mathrm{kN}$ tableting force.

Figure 3.12 Compactibility graph for tablets produced using varying ratios of mannitol and MCC, using either 20 or 100 bar RC pressures and using $20 \mathrm{kN}$ tableting force.

Figure 3.13 X-ray tomography images of tablets with varying ratios of mannitol and MCC, which were compacted using $5 \mathrm{kN}$ tableting force and 20 and 100 bar RC pressure granules. The images presented were obtained from the centre of the tablet and the colour altered for the purpose of presentation 
to highlight any variation in the internal densities. Examples of the granular material and pores present within the tablet are highlighted. 81

Figure 3.14 X-ray tomography images of tablets with varying ratios of mannitol and MCC, which were compacted using $20 \mathrm{kN}$ tableting force and 20 and 100 bar RC pressure granules. The images presented were obtained from the centre of the tablet and the colour altered for the purpose of presentation to highlight any variation in the internal densities. 83

Figure 3.15 Schematic of granule packing after tableting depending on the roller compactor pressure.

Figure 4.1 Schematic of USP LIXELL setup.

Figure 4.2 Average $\mathrm{D}_{10}$ and $\mathrm{D}_{50}$ values for tablets produced using varying formulations and processing parameters. Observed using the LIXELLQICPIC system.

Figure 4.3 Man0:MCC100 tablet produced using 20 bar granules compacted using $20 \mathrm{kN}$ tableting force a) during disintegration at the maximum swelled size and b) after the disintegration measurement ended. 95

Figure 4.4 Man0:MCC100 tablet produced using 100 bar granules compacted using $20 \mathrm{kN}$ tableting force a) during disintegration at the maximum swelled size and b) after the disintegration measurement ended. 96

Figure 4.5 The change in $\mathrm{D}_{10}$ and $\mathrm{D}_{50}$ values with time for Man100:MCC0 tablets produced using a) 20 bar RC pressure, $5 \mathrm{kN}$ tableting force; b) 100 bar RC pressure, $5 \mathrm{kN}$ tableting force; c) 20 bar RC pressure, $20 \mathrm{kN}$ tableting force; d) 100 bar RC pressure, $20 \mathrm{kN}$ tableting force

Figure 4.6 The change in $\mathrm{D}_{10}$ and $\mathrm{D}_{50}$ values with time for Man75:MCC25 tablets produced using a) 20 bar RC pressure, $5 \mathrm{kN}$ tableting force; b) $100 \mathrm{bar}$ RC pressure, $5 \mathrm{kN}$ tableting force; c) 20 bar RC pressure, $20 \mathrm{kN}$ tableting force; d) 100 bar RC pressure, $20 \mathrm{kN}$ tableting force 98

Figure 4.7 The change in $\mathrm{D}_{10}$ and $\mathrm{D}_{50}$ values with time for Man25:MCC75 tablets produced using a) 20 bar RC pressure, $5 \mathrm{kN}$ tableting force; b) $100 \mathrm{bar}$ 
RC pressure, $5 \mathrm{kN}$ tableting force; c) 20 bar RC pressure, $20 \mathrm{kN}$ tableting force; d) 100 bar RC pressure, $20 \mathrm{kN}$ tableting force

Figure 4.8 The change in $\mathrm{D}_{10}$ and $\mathrm{D}_{50}$ values with time for Man0:MCC100 tablets produced using a) 20 bar RC pressure, $5 \mathrm{kN}$ tableting force; b) 100 bar RC pressure, $5 \mathrm{kN}$ tableting force; c) 20 bar RC pressure, $20 \mathrm{kN}$ tableting force; d) 100 bar RC pressure, $20 \mathrm{kN}$ tableting force 101

Figure 4.9 Number of entrained particles for Man100:MCC0 tablets produced using varying processing parameters 102

Figure 4.10 Number of entrained particles for Man75:MCC25 tablets produced using varying processing parameters. 103

Figure 4.11 Number of entrained particles for Man25:MCC75 tablets produced using varying processing parameters. 105

Figure 4.12 Number of entrained particles for Man0:MCC100 tablets produced using varying processing parameters. 106

Figure 4.13 Relative mannitol release profile for Man100:MCC0 tablets, produced using various processing parameters, measured using ${ }^{1} \mathrm{H}$ NMR spectroscopy 108

Figure 4.14 Relative mannitol release profile for Man75:MCC25 tablets, produced using various processing parameters, measured using ${ }^{1} \mathrm{H}$ NMR spectroscopy.

Figure 4.15 Relative mannitol release profile for Man25:MCC75 tablets, produced using various processing parameters, measured using ${ }^{1} \mathrm{H}$ NMR spectroscopy..... 110

Figure 4.16 The relative mannitol release at $60 \mathrm{~s}$ for Man100:MCC0, Man75:MCC25 and Man25:MCC75 tablets produced using 20 and 100 RC pressure and 5 and $20 \mathrm{kN}$ tableting force.

Figure 5.1 Schematic and image of the Flow Cell set-up. 
Figure 5.2 Images taken during Flow Cell analysis of tablet disintegration for tablets produced using $5 \mathrm{kN}$ tableting force for a) Man100:MCC0, RC pressure 20 bar; b) Man100:MCC0, RC pressure 100 bar; c) Man75:MCC25, RC pressure 20 bar; d) Man75:MCC25, RC pressure 100 bar; e) Man50:MCC50, RC pressure 20 bar; f) Man50:MCC50, RC pressure 100 bar; g) Man25:MCC75, RC pressure 20 bar; h) Man25:MCC75, RC pressure 100 bar.

Figure 5.3 Images taken during Flow Cell analysis of tablet disintegration for tablets produced using $20 \mathrm{kN}$ tableting force for a) Man100:MCC0, RC pressure 20 bar; b) Man100:MCC0, RC pressure 100 bar; c) Man75:MCC25, RC pressure 20 bar; d) Man75:MCC25, RC pressure 100 bar; e) Man50:MCC50, RC pressure 20 bar; f) Man50:MCC50, RC pressure 100 bar; g) Man25:MCC75, RC pressure 20 bar; h) Man25:MCC75, RC pressure 100 bar.

Figure 5.4 Schematic of the water flow and velocity acting on the tablet inside the Flow Cell. 125

Figure 5.5 The change in the normalised tablet area with time for tablets with varying ratios of mannitol and MCC granules which were produced using RC pressure of 20 and 100 bar and a tableting force of $5 \mathrm{kN}$.

Figure 5.6 The total number of released particles with time for tablets with varying ratios of mannitol and MCC granules which were produced using $\mathrm{RC}$ pressure of 20 and 100 bar and a tableting force of $5 \mathrm{kN}$ 129

Figure 5.7 The change in the normalised tablet area with time for tablets with varying ratios of mannitol and MCC, produced using a tableting force of 20 $\mathrm{kN}$.

Figure 5.8 The total number of released particles with time for tablets with varying ratios of mannitol and MCC, produced using a tableting force of 20 $\mathrm{kN}$.

Figure 6.1 USP Basket-Rack assembly. 140 
Figure 6.2 Volume-weighted granule $\mathrm{D}_{10}, \mathrm{D}_{50}$ and $\mathrm{D}_{90}$ data for the various formulations.

Figure 6.3 The tensile strength $\sigma_{\mathrm{T}}( \pm \mathrm{SD})$ and porosity $\varepsilon_{\mathrm{T}}( \pm \mathrm{SD})$ of tablets. Granules were produced using 20 or 50 bar roller compactor hydraulic pressure; the granules were then compressed using a tableting force of 15 or $25 \mathrm{kN}$. 143

Figure 6.4 Binary images obtained during tablet disintegration at $10 \mathrm{~min}$ intervals for tablets a) ExMCC-A; b) ExMan-A; c) ExMan-B; d) ExMan-C. . 147

Figure 6.5 a) 2D tablet area vs time during the disintegration of tablets produced using granules compressed at 20 bar hydraulic pressure in a roller compactor. A tableting force of $25 \mathrm{kN}$ was used to compact the excess MCC and excess mannitol granules, respectively; b) the number of particles released for the aforementioned tablets. 148

Figure 6.6 a) 2D tablet area vs time during the disintegration of tablets produced using granules compressed using 20 and 50 bar roller compactor pressure, compacted at a tableting force of $15 \mathrm{kN}$. b) The number of particles released for the aforementioned tablets. 151

Figure 6.7 a) 2D tablet area vs time during the disintegration of tablets produced using granules compressed using 20 bar roller compactor pressure, compacted at a tableting force of 25 or $15 \mathrm{kN} \mathrm{b}$ ) The number of particles released for the aforementioned tablets. 153

Figure 6.8 Average $\mathrm{D}_{10}, \mathrm{D}_{50}$ and $\mathrm{D}_{90}$ values for the particles released from the tablets with time. 154 


\section{List of Tables}

Table 2.1 General categories of the excipients used in pharmaceutical tablet formulations, their function and the ranges of $w / w \%$ typically used in formulations (Rowe et al., 2009).

Table 2.2 Tablet formulation 46

Table $3.1 \mathrm{D}_{10}, \mathrm{D}_{50}$ and $\mathrm{D}_{90}$ values and the solubility data for the filler materials which were examined (Rowe et al., 2009)

Table 3.2 Formulations and processing conditions used to produce binary mixture granules with abbreviations.

Table 3.3 The effective angle of internal friction $\left(\delta_{\mathrm{E}}\right)$, the angle of wall friction $\left(\varphi_{\mathrm{w}}\right)$ and compressibility $(\mathrm{K})$ values.

Table 3.4 The values for the stress applied when roller compaction pressures of 20 and 100 bar are used during granulation for Man100:MCC0 and Man0:MCC100 formulations.

Table 3.5 The values for the force used during tableting, and the equivalent stress

Table 4.1 Formulations and processing conditions used to produce binary mixture granules with abbreviations.

Table 5.1 The formulations analysed and their abbreviations. RC pressure and tableting force is also specified.

Table 6.1 The abbreviations for the placebo formulations. 138

Table 6.2 The RC pressures and tableting forces used to produce tablets. 139

Table 6.3 Disintegration data obtained via USP analysis. 145 


\section{Glossary}

API

Active Pharmaceutical Ingredient.

BINDER

A liquid or solid component in a tableting formulation which binds the other components together during compaction.

COMPACTIBILITY The relationship between the tensile strength and porosity of a compact.

COMPRESSIBILITY The change in a compact's tensile strength due to the applied compaction pressure.

DENSITY

A measure of mass per volume.

DISINTEGRANT An excipient that is added to a tablet formulation to promote the break-up of the tablet into smaller fragments.

DisinTEGRATION The break-up of the tablet into smaller fragments with a larger surface area.

Dissolution A solute (i.e. tablet API) dissolving into a solvent (i.e. liquid such as water or stomach acid).

EXCIPIENT

A component which is the vehicle or medium for the API, which serves a specific purpose (e.g. bulking, binding, aiding disintegration/dissolution).

FILLER An excipient which adds bulk to the tablet formulation to ease ingestion by the consumer.

GRANULE A small compact of powder produced either through application of stress or through agglomeration.

FORMULATION A mixture prepared according to a certain formula.

Placebo A substance which contains no API and therefore has no therapeutic value, which can function as a control. 
LOSS IN A reduction in the ability of a material to plastically deform

REWORKABILITY due to the material being "worked" multiple times.

LUBRICANT An excipient which reduces friction, and therefore sticking of the formulation material to the production machinery.

Porosity A measure of the void space in a material as a percentage.

RIBBon A compact produced by application of stress between two rollers during roller compaction granulation. A precursor to granules.

TABLET A solid unit dosage produced by application of stress to powder or granular material, which may contain a formulation or singular material. 


\section{Chapter 1. Introduction}

\subsection{Roller Compaction Granulation}

Granulation is a widely used technique for the conversion of primary powder particles into larger, agglomerated granules. Granules are preferred rather than powders in the pharmaceutical industry, due to the improvement in bulk granule properties such as flowability, wettability, bulk density and homogeneity of the components within the formulation. Additionally, granulated material is easier to transport and handle as the percentage of fine particles is reduced. This is beneficial, for example, when using active pharmaceutical ingredients (API) which are toxic when inhaled.

Various methods can be used to granulate powders, each with their own advantages. The granules produced will have different properties, such as size, size distribution, strength, porosity and dissolution, depending on the granulation technique used. Generally the techniques are characterised as either wet or dry granulation. This research focuses on roller compactor dry granulation.

Dry granulation relies on using an applied stress to densify and bond primary particle powders together into a denser compact also known as a ribbon. Roller compaction is a predominant dry granulation technique used in the pharmaceutical industry as it allows for continuous granulation. This means that a more uniform final granule product can be obtained in a continuous line with the elimination of the possibility of batch variation (Kleinebudde, 2004). Additionally, roller compaction allows for the granulation of materials which are water or temperature 
sensitive, which may be beneficial when granulating certain API. There is no need to use a liquid binder and therefore, the drying step is also eliminated meaning that the cost is lowered (Guigon et al., 2007). A downside to roller compaction is that a high percentage of fines, i.e. un-compacted powder, may be produced during granulation.

Roller compactor designs vary depending on the manufacturer. Commercially available roller compactors may have rollers which are mounted in a vertical, inclined or horizontal setting, where the former relies on gravity for the feeding of powder and the latter two rely on a feed auger to convey the powder between the rollers (Guigon et al., 2007).

Roller compaction offers the ability to control various processing parameters during granulation. These can include screw feed rate, roller speed and crusher speed, mesh screen size. The main parameter which can be altered during roller compaction is the stress applied to the material when it is between the rollers. It is possible to control the thickness of the ribbon and the size, strength and porosity of the granules produced via variation of these process parameters (Perez-Gandarillas et al., 2015, Kazemi et al., 2017, Pérez Gago et al., 2017). This is beneficial in pharmaceutical formulation development as it offers a level of control over the final tablet properties.

While roller compaction is a preferred technique for the production of granules, there are inherent issues that can occur. Loss in compressibility of granules due to application of stress is a major issue which is observed in roller compaction. A higher pressure leads to better compacted ribbons and granules, however the consequent tablets exhibit a lower tensile strength (Farber et al., 2008). However, with the application of a lower stress leads to an increase in the 
percentage of uncompacted powder material, otherwise known as fines (Inghelbrecht et al., 1997). If a high percentage of fines are obtained then the material may need to be re-compacted, which adds additional cost and time to the granulation process and, again, a loss in material compressibility (Inghelbrecht and Remon, 1998a, Bultmann, 2002). Therefore, there is a trade-off between the ribbon/granule quality and the tablet quality which must be considered.

\subsection{Tableting}

Tablets are the predominant solid dosage forms used in the pharmaceutical industry as they allow for precise administration of a drug load with little variation between batches. Tablets also have the benefit of being able to withstand transportation and storage following production (Cooper and Rees, 1972).

To produce a tablet, material is subjected to a second stress which compresses it into a "slug". In the pharmaceutical industry, production of a tablet can be carried out in one of two ways. The powder mixture can be directly compressed or it can be granulated and then the granules are compressed into tablets. The process which is used is dependent on the properties of the powder mixture. The powder mixture must be free-flowing and not prone to segregation to be suitable for direct compression. However, as outlined previously, granulation offers improvement of these properties, and additionally reduces the percentage of fines.

\subsection{Tablet Disintegration and Dissolution}

In the pharmaceutical industry, dissolution and disintegration are essential tablet properties which are tested when a new tablet formulation is hypothesised. Firstly, the breakage of larger agglomerates into smaller particles is termed as tablet disintegration (see Figure 1.1). The definition of tablet dissolution can be described 
as the solid soluble components dissolving into solution (see Figure 1.2). Dissolution of the soluble component occurs from the surface of the tablet, therefore disintegration aids the dissolution process by providing a larger surface area (Fassihi, 1986).
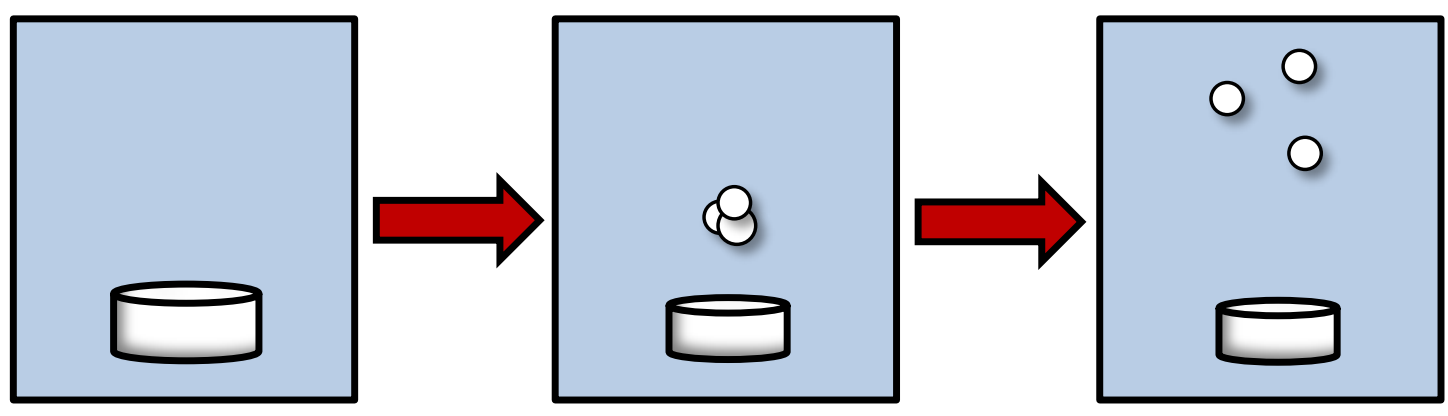

Figure 1.1 Schematic of tablet disintegration showing the breakage of agglomerates released from a tablet.
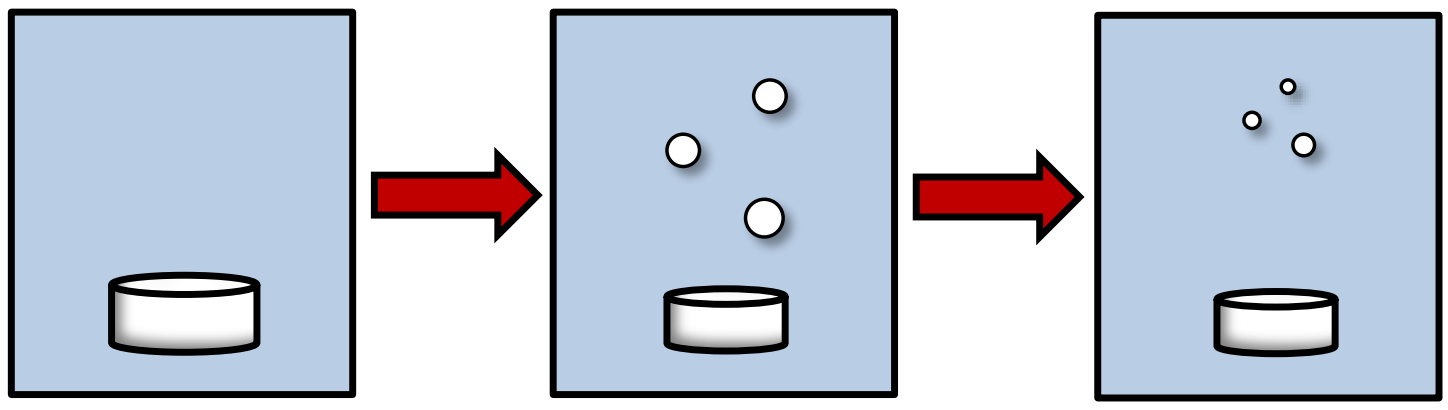

Figure 1.2 Schematic of tablet dissolution showing the particles from a tablet dissolving into solution.

The various processes, such as wicking, wetting and penetration, occurring during dissolution and disintegration occur in simultaneously to perpetuate the break-up of the tablet and release the soluble material (Caramella et al., 1988, Lowenthal, 1972, Delalonde and Ruiz, 2008). Furthermore, pharmaceutical tablets can contain numerous excipients, each of which behave differently in solution, for example hydrophobic or hygroscopic powders (Wan and Prasad, 1988). It has also been found that process parameters used to produce the tablet can also affect the 
dissolution-disintegration behaviour (Merkku et al., 1994). During analysis the tablet is analysed as a whole entity, and little knowledge is gained as to how each excipient alters the tablet dissolution-disintegration profile.

In the pharmaceutical industry tablet dissolution and disintegration tests are carried out at first in vitro as an important initial indicator of the probable tablet performance in vivo. A standard dissolution-disintegration testing apparatus is used to determine whether a tablet performs suitably (Donauer and Löbenberg, 2007). A commonly used dissolution apparatus is the USP II (Figure 1.3), which comprises a vessel containing a paddle which agitates the solution, a mesh basket for the placement of the tablet, and a probe (e.g. conductivity or UV) capable of measuring the concentration of the soluble material in solution (Azarmi et al., 2007).

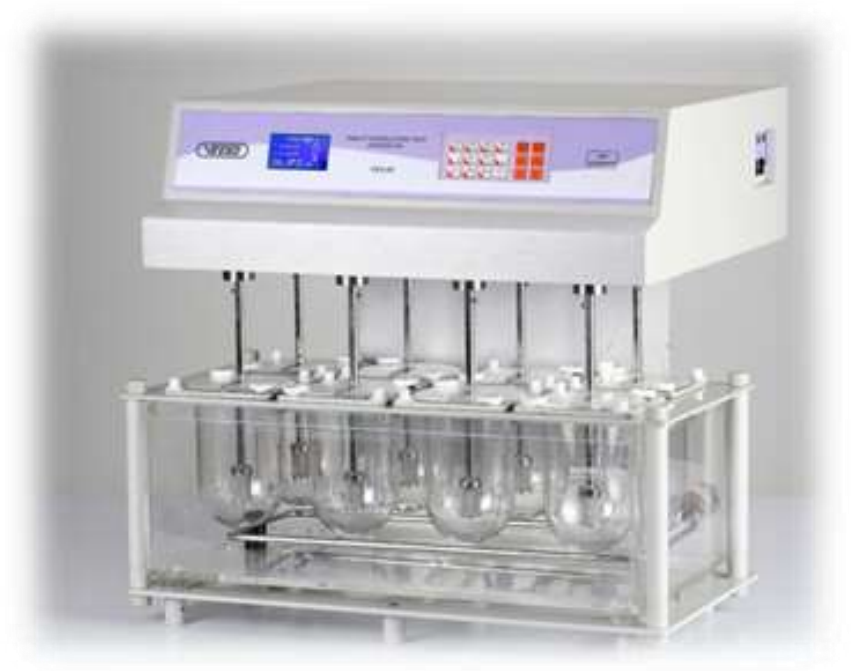

Figure 1.3 Image of a USP II apparatus with 8 vessels.

Tablet dissolution is measured by the time taken for the concentration of the relevant soluble material to reach a set end point, e.g. for a fast-release tablet $90 \%$ API concentration in $30 \mathrm{~min}$ would be expected. Disintegration is simply measured by the time taken for a tablet to fully pass through the mesh basket. Tablets must 
conform to the standards set by the appropriate governing body (Williams, 2006). The data is analysed and if the tablet does not perform as expected then the tablet formulation is re-examined and the tablet formulation is altered in an attempt to manipulate the tablet to behave as required. This cycle may occur repeatedly until an ideal formulation is reached which can go on to the next testing stage of formulation development. However this "data-driven", pass or fail approach does not provide any knowledge as to why the tablet failed. An additional problem with standard testing is that disintegration and dissolution tests are separate and are not related to each other. The dissolution is measured with time, whereas the disintegration is a singular time point following complete tablet disintegration. Additionally, tablet formulations generally have more than one soluble component and conductivity and UV may not be able to differentiate between them. Therefore, generally the dissolution measurement is only an indication of the concentration of the API in solution.

\subsection{Aims and Objectives}

This research aims to analyse the effects of varying the formulation and two fundamental stresses which are applied to a material during the process of producing a tablet via roller compaction granulation.

For this research a brittle material and a ductile material have been chosen so that these properties may be examined. The effects of varying the two processing stresses will be determined in terms of the tablet compactibility, i.e. the tensile strength and porosity. Additionally, X-ray tomography will be utilised to examine the ribbons and tablets produced. This will also allow for examination of the tablet 
internal structure and to relate this to the "loss in compressibility" of tablets, and consequently the tablet disintegration behaviour.

The tablet disintegration behaviour will be examined using 3 different methods; the standard USP method, the Flow Cell imaging method and the off-line LIXELL particle imaging method. The dissolution behaviour will also be evaluated off-line using ${ }^{1} \mathrm{H}$ NMR spectroscopy.

The LIXELL imaging method, used in conjunction with the NMR method, is a measurement technique which is able to monitor the particle release and dissolution with time. The Flow Cell disintegration analysis method is capable of visualising the change in tablet area and the particle release in real-time.

\subsection{Thesis Layout}

In Chapters 1 and 2 a background in roller compaction granulation and tablet disintegration have been outlined. Literature has been examined to exhibit the current understanding of the effects of granulation and other factors which affect tablet disintegrations. Alternative methods to analyse tablet disintegration have also been evaluated.

In Chapter 3 the characterisation of the roller compacted material has been presented. This includes the characterisation of the ribbons, granules and tablets produced using varying roller compaction pressures and tableting forces. The tablet compactibility and the phenomenon of "loss in compressibility" will be examined depending on the granulation and tableting stresses used. The internal structure of tablets will be analysed.

Chapter 4 explores the use of a modified USP apparatus connected to a LIXELL particle imaging method to analyse tablet disintegration. The effect of 
varying $\mathrm{RC}$ pressure and tableting force was examined and linked to the findings in the previous chapters. ${ }^{1} \mathrm{H}$ NMR was used to determine the dissolution behaviour of mannitol.

In Chapter 5 the Flow Cell method was examined in depth to determine the effect of varying the stresses applied during roller compaction and tableting. The effects were observed in terms of the change in tablet area and the particle release.

In Chapter 6 a study was carried out to compare the capabilities of the Flow Cell method and the standard USP method. It was examined whether the Flow Cell method was able to elucidate additional information regarding the processes which occur during tablet disintegration. It was also examined whether the Flow Cell method was capable of detecting the changes in tablet disintegration when the processing parameters were varied.

Chapter 8 concludes the research and outlines the main findings. The tablet compactibility was clarified in terms of the stresses applied during RC and tableting. The tablet disintegration behaviour observed using the LIXELL, the Flow Cell method and USP method was related to the tablet properties. The dissolution trends were also examined. 


\section{Chapter 2. Literature Review}

\subsection{Introduction}

Understanding the mechanisms occurring during the disintegration of tablets is vital, particularly in the pharmaceutical industry due to the precision required when administering drug loads. Quality by design $(\mathrm{QbD})$ within the pharmaceutical industry requires that the preparation of solid dosage tablets must be carried out within strict and well-planned conditions so that the tablet releases the active pharmaceutical ingredient (API) in a precise manner when ingested (Azarmi et al., 2007). Variation in the materials within the formulation and the processing parameters, i.e. during granulation or tableting, affects the disintegration behaviour. Understanding the link between each variation and the effect on the tablet disintegration could lead to more proficient tablet design. Additionally, by using novel techniques the disintegration process itself can be understood in more depth.

This Chapter will review the current research and understanding surrounding the effects of variation of the tablet formulation, roller compaction parameters and tableting parameters on tablets, and consequently tablet disintegration and dissolution. A focus will be put on the phenomenon of "loss in compressibility" in tablets following roller compaction and tableting to determine the current understanding. 
It will also cover the alternative techniques currently being developed in research which analyse tablet disintegration and what information is available to be gained by them. This research focuses on placebo formulations and therefore research concerning APIs is beyond the scope of this work.

\subsection{Factors Affecting Disintegration and Dissolution}

\subsubsection{Pharmaceutical Tablet Formulation}

Pharmaceutical solid dosage systems consist of various excipients which each play a role in the disintegration and dissolution tablet. Table 2.1 shows the different excipient components which are commonly used in pharmaceutical tablet formulations. Generally a solid dosage tablet will contain an API and additional pharmacologically inert excipient materials, depending on the functionality of the tablet (Pifferi et al., 1999). The most important factor that must be considered is the efficient transport of the API from the solid tablet into the solute. To enable the precise dissolution of API, the remaining inert excipients each play important roles in aiding its precise release. Some APIs are poorly soluble and therefore the formulation would require carefully selected excipients which promote disintegration and dissolution so that the function of the API is able to be carried out (Buggins et al., 2007, Desai et al., 1996, Loftsson, 2015). These materials aid in the disintegration of the tablet into smaller fragment which provides a larger surface area for the dissolution of the API (Desai et al., 2016).

Disintegrants are added to tablet formulations to encourage the disintegration of the tablet when ingested which then promotes the dissolution of the API. Various types of disintegrants are used for oral dosage tablets depending on the required 
release time (Rowe et al., 2009). In other words, for fast release tablets superdisintegrants are used which are capable of achieving full tablet disintegration within $30 \mathrm{~s}$ (Sunada and Bi, 2002). Some disintegrants release the tablet drug load in a time frame of $5-30 \mathrm{~min}$, around the time that the tablet reaches the stomach.

Table 2.1 General categories of the excipients used in pharmaceutical tablet formulations, their function and the ranges of w/w\% typically used in formulations (Rowe et al., 2009).

\begin{tabular}{|c|c|c|}
\hline Excipient & Function & $\begin{array}{l}\text { Approx. w/w\% } \\
\text { used in industry }\end{array}$ \\
\hline API & The active pharmaceutical ingredient & Variable \\
\hline Filler & $\begin{array}{c}\text { Primarily provides the bulk of the tablet mass, } \\
\text { also improves flowability }\end{array}$ & $70-85$ \\
\hline Disintegrant & $\begin{array}{l}\text { Aids in the breakage of the tablet into smaller } \\
\text { particles with a larger surface area for dissolution } \\
\text { to occur }\end{array}$ & $2-5$ \\
\hline Binder & $\begin{array}{l}\text { Helps to maintain the structure of the tablet } \\
\text { during production, transport and storage. }\end{array}$ & $2-5$ \\
\hline Lubricant & $\begin{array}{l}\text { Mainly to aid in the upkeep of the equipment } \\
\text { used to produce the tablets as powders tend to } \\
\text { stick and become difficult to remove }\end{array}$ & $1-2$ \\
\hline Other & $\begin{array}{c}\text { Such as stabilisers, pH modifiers, encapsulating } \\
\text { agents, gelling agent }\end{array}$ & $1-10$ \\
\hline
\end{tabular}

Binders and lubricants are added to a pharmaceutical formulation mainly to assist in the processing steps which produce the tablet. In other words, during granulation and tableting binders will help to agglomerate the primary powder and promote the formation of strong granules and tablets (Li et al., 2004). In some cases the materials used are cohesive and stick to the granulation and tableting machinery. Lubricants help to prevent material wastage due to adhesion by 
reducing friction during processing and improve granule flow (Miller and York, 1988, Miguélez-Morán et al., 2008). However, it has been found that addition of lubricant negatively affects tablet disintegration and dissolution. One reason is due to the hydrophobicity of lubricants causing water penetration to be hindered and consequently slowing disintegration (Wang et al., 2010). Additionally, it has been reported that tablets which contain lubricant are weakened due to the reduced cohesiveness (He et al., 2007). It should be noted that the extent of the effect of lubricant on the granular and tablet properties depend on the excipient properties (Otsuka et al., 2004).

Fillers are used to bulk out the tablet's content as the API may only occupy a small percentage of the total tablet weight (Loftsson, 2015). Fillers are generally inert and may possess additional qualities such as good taste and mouthfeel or disintegrant-like properties which are additional functions of their use in a pharmaceutical tablet formulation (Rowe et al., 2009). The filler used in a formulation will be selected depending on the function of the tablet and the compatibility of the other components. In this research microcrystalline cellulose and mannitol will be examined as they are widely used in pharmaceutical formulations (Ohrem et al., 2014, Rowe et al., 2009).

Microcrystalline cellulose (MCC) is widely used in industry as a filler material due to its inertness and ductile properties, and will be analysed in this research (Rowe et al., 2009). The ductile nature of MCC allows for a high level of compression without the material undergoing brittle fracture. However, MCC is prone to a significant loss in tensile strength even at low pressures. Additionally, it is also a hygroscopic material and it has been found that moisture content within MCC influences properties such as tensile strength (Jivraj et al., 2000). MCC is also 
known to have the ability to absorb water and swell, which suggests that it can also be used as a disintegrant (Thoorens et al., 2014).

Mannitol is a non-hygroscopic filler material which makes it an ideal filler for use with moisture sensitive APIs. Mannitol is a brittle which means that the loss in compressibility even at high compaction pressures is limited (Wu and Sun, 2007, Rowe et al., 2009). It is commonly used in diabetic medications as, unlike some fillers such as sorbitol, it does not affect blood sugar levels in humans but still offers a "sweet" taste and good mouth-feel (Wagner et al., 2015).

\subsubsection{Granulation and Tableting}

During roller compaction (RC) granulation, material is compacted into ribbons. These ribbons are then crushed into granules. This first stress applied to the material in the process of producing tablets is the RC pressure used to compact the primary powder into ribbons. The second stress applied to the material is during tableting.

Inghelbrecht and Remon conducted research in which the variation of the excipient and RC pressure were examined in terms of the tablet properties (Inghelbrecht and Remon, 1998b). Various grades of MCC were granulated with varying amounts of ibuprofen via roller compaction. Roller compaction pressures of 2.3 and $6.9 \mathrm{MPa}$ were used, and the tableting force was kept constant at $5.88 \mathrm{kN}$. It was determined that after granulation, tableting caused the various grades of MCC to undergo work hardening, in other words the deformability of the material decreased. It was reported that a higher RC pressure leads to the production of "better" ribbons and granules. It must be assumed that by "better" the authors 
mean to say more well-compacted. However, it was found that the consequent tablet mechanical strength was poor.

Much research has been conducted which agrees that the application of a higher RC pressure leads to increased ribbon and granule strength and decreased porosity (Chang et al., 2008, Allesø et al., 2016). It has also been reported that more porous tablets are more likely to disintegrate quickly due to the increased rate of penetration due to the higher number of available pores (Lowenthal, 1972).

An increased RC pressure leads to less fines being produced, therefore the size distribution is improved (Wagner et al., 2013). However, the tablet tensile strength is widely documented to reduce with increasing RC pressure (Perez-Gandarillas et al., 2016, Farber et al., 2008, He et al., 2007, Hadžović et al., 2011). This is generally termed as the "loss in compressibility" of a material. Tablet tensile strength in terms of the tablet porosity is termed as the compactibility (Leuenberger, 1982). An example for the reduction in tablet tensile strength due to RC pressure is shown in Figure 2.1. It should be noted that for the filler materials used in this research, roller compaction reduces the tensile strength at any RC pressure; directly compacted material produces the strongest tablets for these materials (Rowe et al., 2009). 


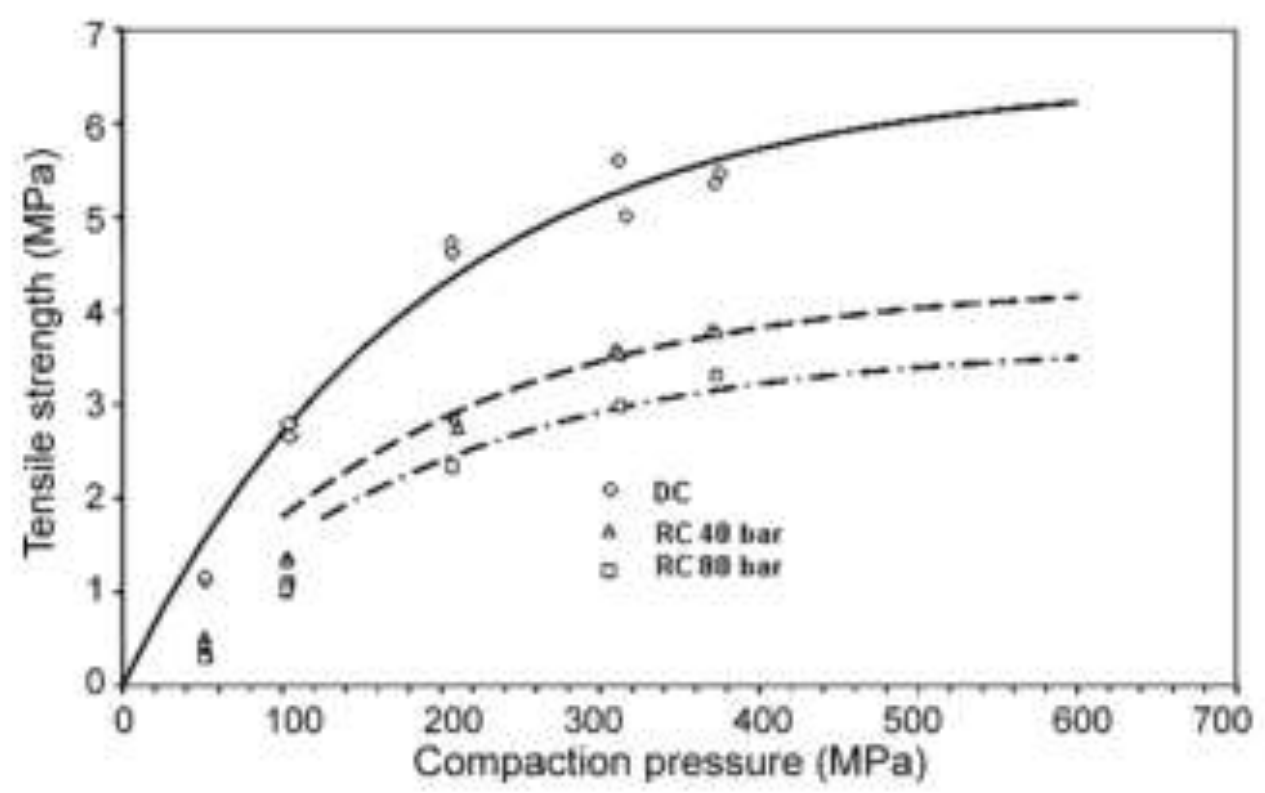

Figure 2.1 Tablet tensile strength for a formulation containing $72 \%$ Avicel PH102, $24 \%$ lactose monohydrate, $3 \%$ croscarmellose sodium and $1 \%$ magnesium stearate. Tablets were produced using various compaction pressures using granulated material (40 or 80 bar RC pressure), or directly compressed from the powder (Farber et al., 2008).

Loss in compressibility of a material is a key issue with roller compaction. Some of the available literature suggests that the phenomenon being governed by the ribbon density. He et al. utilised RC to produce microcrystalline cellulose (MCC) tablets by compacting them at varying RC pressures (He et al., 2007). The tablet tensile strength and porosity were studied in terms of the ribbon solid fraction (SF); it was found that higher SF modestly decreased the tensile strength and porosity of the tablet. Similarly, Herting and Kleinebudde examined the compactibility was studied in terms of the ribbon porosity (Herting and Kleinebudde, 2007). It was found that a lower ribbon porosity led to weaker tablets, and that the tablet porosity was largely unaffected.

Later research reported that the loss in compressibility of tablets following RC was due to the variation in particle size, depending on the RC pressure used. A 
smaller granule size led to a higher specific surface area, and consequently higher tensile strength in the respective tablets (Herting and Kleinebudde, 2008).

Figure 2.2 shows that Sun and Himmelspach also attributed that the reduced compactibility of roller compacted granules to the "size enlargement" of the particles which occurs during granulation (Sun and Himmelspach, 2006). It was reported that at "zero porosity" the tablet tensile strength still decreased depending on the number of compactions used. Mitra et al. utilised monodisperse granules to attribute the granule solid fraction to the consequent tablet tensile strength rather than the granule size (Mitra et al., 2015). However, in the research previously conducted the reduced compressibility phenomenon has not been examined in terms of the stresses applied during granulation and tableting. More importantly, the research has focussed largely on the granule size or density rather than granule strength and the bonding within a tablet.

The tablet compressibility varies depending on the material and its properties (Tye et al., 2005). As previously mentioned, application of a higher compaction pressure during tableting has shown to increase the tablet tensile strength and decrease the tablet porosity (Sinka et al., 2009, Adolfsson and Nyström, 1996). This is due to the higher pressure leading to better particle-particle bonding and densification of the material (van der Voort Maarschalk et al., 1996). 


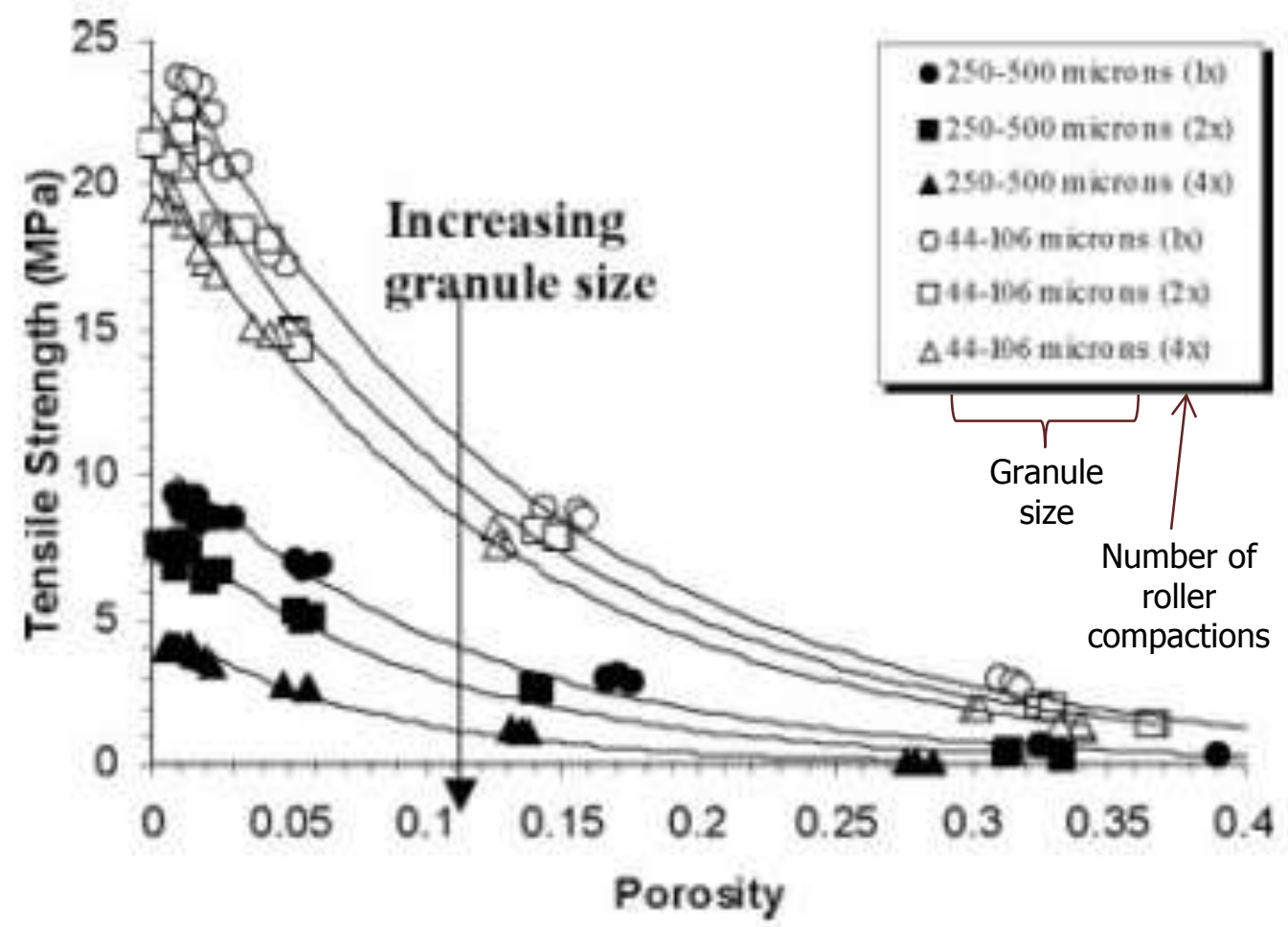

Figure 2.2 Tablet compactibility graphs of sieved MCC granules produced by different numbers of roller compactions, highlighting that larger granules exhibit lower compactibility (Sun and Himmelspach, 2006).

Hadžović et al. reported that MCC tends to exhibit a great loss in compressibility and compactibility (Hadžović et al., 2011). Tablets were produced using RC granules compacted at 20 or 30 bar pressure and also by direct compression of the powder material. Varying forces were used to compress the granular material and the powder material into tablets. It was clearly seen that directly compressed tablets were stronger than the tablets produced using ribbons at the same tableting force. Additionally, a higher RC pressure led to a reduction in the tablet tensile strength. It was explained that this is an inherent property of plastic materials.

$\mathrm{Wu}$ et al. examined the effect of the brittleness on the tabletability, i.e. the change in tablet tensile strength with compaction pressure used (Wu and Sun, 2007). 
Mannitol was one of the materials which were examined in this research. This research also assumed that the size enlargement of granules is related to the loss in tabletability, as previously suggested by Sun and Himmelspach (Sun and Himmelspach, 2006). Therefore, mannitol was granulated using roller compaction and sieved into three size classes, then tabletted.

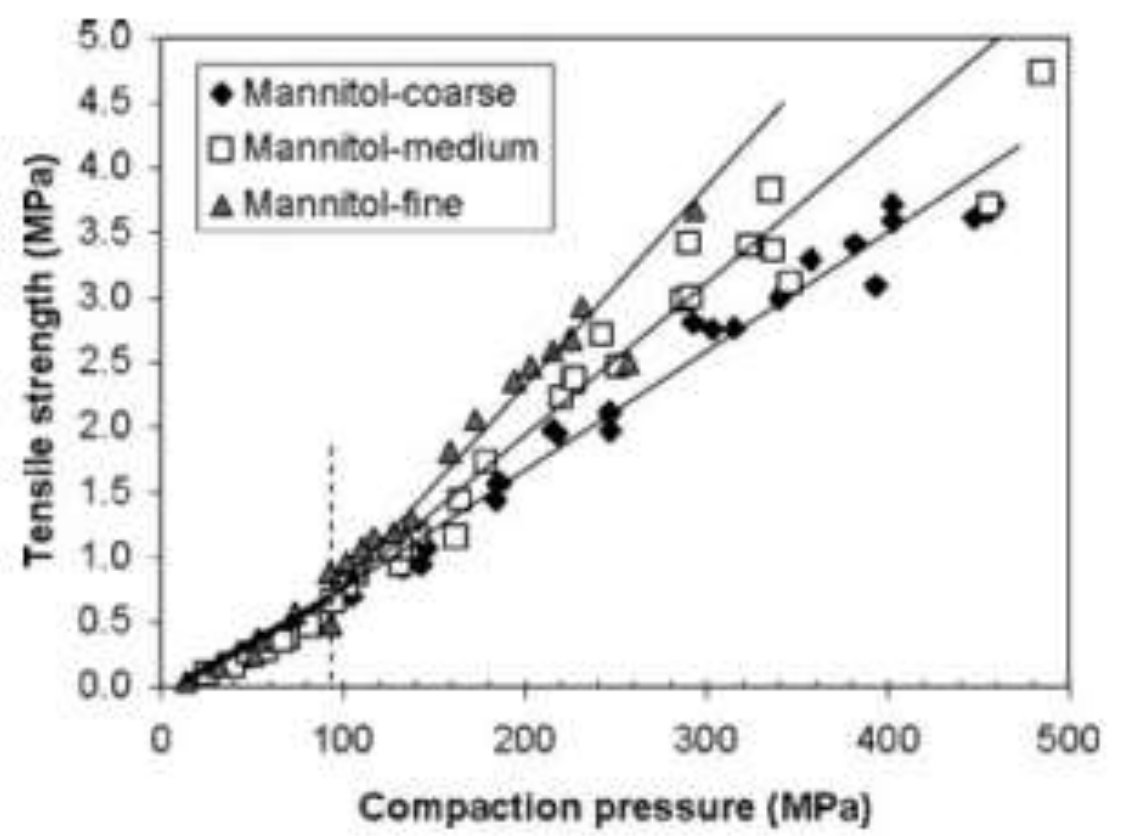

Figure 2.3 Tabletability plot of the three sieved fractions of mannitol granules. Each point is one tablet. (Wu and Sun, 2007)

It was reported that for all three size classes the tabletability exhibited a bilinear change with increasing compaction (see Figure 2.3). At lower compaction pressures the tabletability was almost identical for each size class. After a threshold, depending on the granules size class, a more pronounced difference in the tabletability was visible for each material. It was found that the larger granules produced the weakest tablets. By using linear regression it was found that the difference between the various slopes is significantly different $(p<0.05)$. This research statistically proved that brittle materials also undergo loss in tabletability. 
It was hypothesised that this behaviour in brittle materials is due to the compaction pressure causing brittle fracture of the granular material rather than plastic deformation occurring. Reportedly, larger granules are more likely to undergo brittle fracture whereas smaller granules offer a larger surface area and therefore are more likely to undergo plastic deformation. Therefore a lower tabletability is observed for larger granules. However, it was explicitly stated by the researchers that it was not clear why the divergence in the tabletability occurred with higher compaction pressures (see Figure 2.3).

\subsection{Alternative Analysis Methods}

\subsubsection{Disintegration of tablets}

Following the manufacture of tablets, disintegration testing is used to determine the tablet's viability to deliver the drug load in the required manner. Tablet disintegration occurs due to liquid penetration into the solid dosage system which triggers multiple mechanisms to commence which instigate the tablet to break up (Luginbühl and Leuenberger, 1994). An initial mechanism which occurs is water penetration, which may lead to swelling, causing the breakup of the tablet matrix from inside the tablet. Due to the swelling, deformation of the particles ensues and causes water to be pulled into the tablet pores due to hydrophilic materials such as disintegrants, which disrupts particle-article bonding. Following the water uptake, saturated particles were reported to "repel" each other, leading to further tablet breakage. Erosion occurs, where particles are released from the tablet causing the area of the tablet to shrink in size.

Various factors which affect tablet disintegration have been proposed by research such as wetting time and penetration (Peppas and Colombo, 1989). 
Generally U.S. or European pharmaceutical tablet testing involves measuring the disintegration or the dissolution time of tablets using a standard pharmacopoeia method (Bai et al., 2011). However, it is measured as a finite time point at the end of the tablet disintegration process. No information regarding the mechanisms of disintegration, such as swelling and erosion, is obtained.

In order to gain information regarding the processes occurring during tablet disintegration, analysis methods have been presented in research using various approaches. This was done by measurement of the parameter disintegration force (Colombo et al., 1980, Colombo et al., 1984, Peppas et al., 1989), by using MRI to visualise and image disintegration (Shiko et al., 2011, Quodbach et al., 2014b, Quodbach et al., 2014a, Quodbach and Kleinebudde, 2014) or utilising particle sizing methods to elevate the existing USP apparatus to be capable of linking tablet disintegration and dissolution (Wilson et al., 2012).

By using a texture analyser which mimics a patient's mouth, research was carried out to determine whether accurate disintegration data of rapidly disintegrating tablets could be obtained (Abdelbary et al., 2005). The results of the experiment were compared to results obtained through testing the same tablets using human volunteers. The simulate test was carried out using artificial saliva. Overall, a good correlation was seen with respect to the mouth-feel, penetration, disintegration profile and tablet thickness. While this study was able to replicate the disintegration behaviour with a focus on how it affects the patient, the processes occurring during disintegration were not able to be realised.

Mesnier et al. utilised a novel method to image the disintegration of tablets in a quantifiable manner (Mesnier et al., 2013). A Flow Cell was constructed (see Figure 2.4), consisting of a mesh for the placement of the tablet, a viewing window 
to enable imaging of the tablet. Water was introduced through the lower inlet into the cell and exited through the upper outlet into a basin. This ensured that the released particles from the tablet moved in the same direction as the water flow, and could be imaged without being counted twice. Colour images were obtained during tablet disintegration using a $16 \mathrm{MP}$ camera at a rate of 10 images per second. The images (see Figure 2.4a) were then converted into 8bit grayscale images then thresholded to obtain a binary images that were used to distinguish between released particles, the tablet and the background (see Figure 2.4b). Using the space occupied by the particles in the binary image, the equivalent area of those particles was calculated. The boundaries of the tablet were also established so that the variation of tablet thickness and diameter could also be observed.

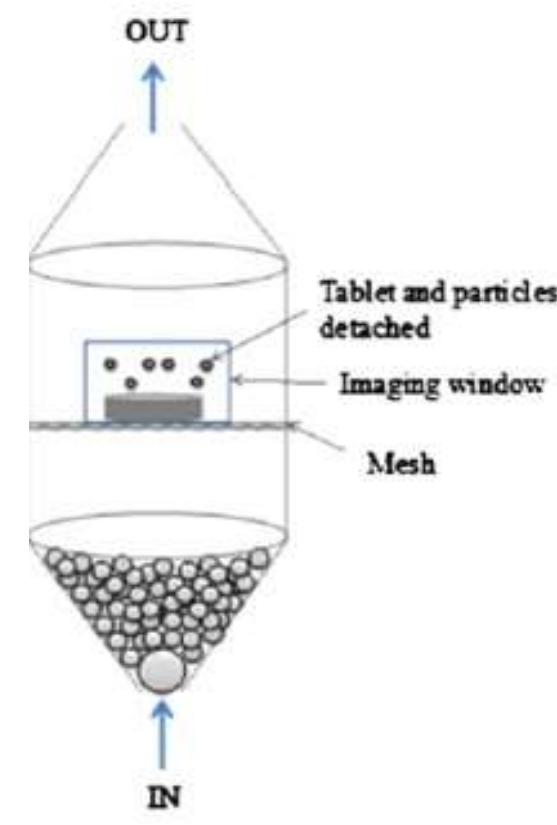

a.

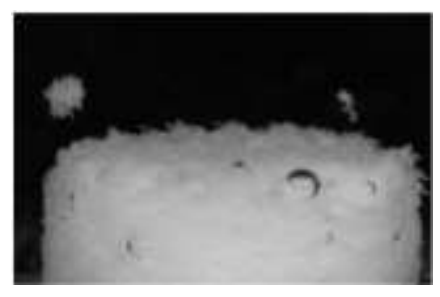

b.

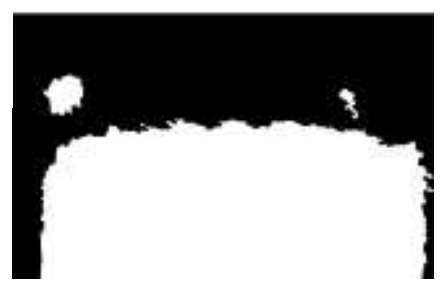

Figure 2.4 Illustration of Flow Cell and a) typical image obtained during disintegration testing using the Flow Cell. b) Converted binary image after thresholding (white - tablet and particles, and black - background) (Mesnier et al., 2013). 
The study aimed to determine the effectiveness of disintegrants on tablet disintegration. Tablets containing (i) $100 \%$ maltodextrin DE 21 and (ii) $98 \%$ maltodextrin DE 21 and $2 \%$ croscarmellose sodium powder mixtures were directly compressed. The tablets produced had the dimensions of $12 \mathrm{~mm}$ in diameter and $6 \mathrm{~mm}$ in height.

As a comparison of the dissolution and disintegration were the main investigative points, tablet characterisation was carried out to identify any other variables. Both the pure maltodextrin tablets and maltodextrin with $2 \%$ disintegrant tablets were calculated to have similar porosities and tablet tensile strengths. Due to the similar porosity and tensile strength values obtained, it was assumed that these properties would not influence the dissolution and disintegration analysis.

Figure 2.5 shows the dissolution profiles obtained from conductivity measurements showed that the tablets containing $2 \%$ disintegrant dissolved faster than pure maltodextrin tablets. But from this study it was clear that no further information is obtainable through dissolution testing via conductivity, such as the concentration and structure of the soluble material in solution at any given time. 


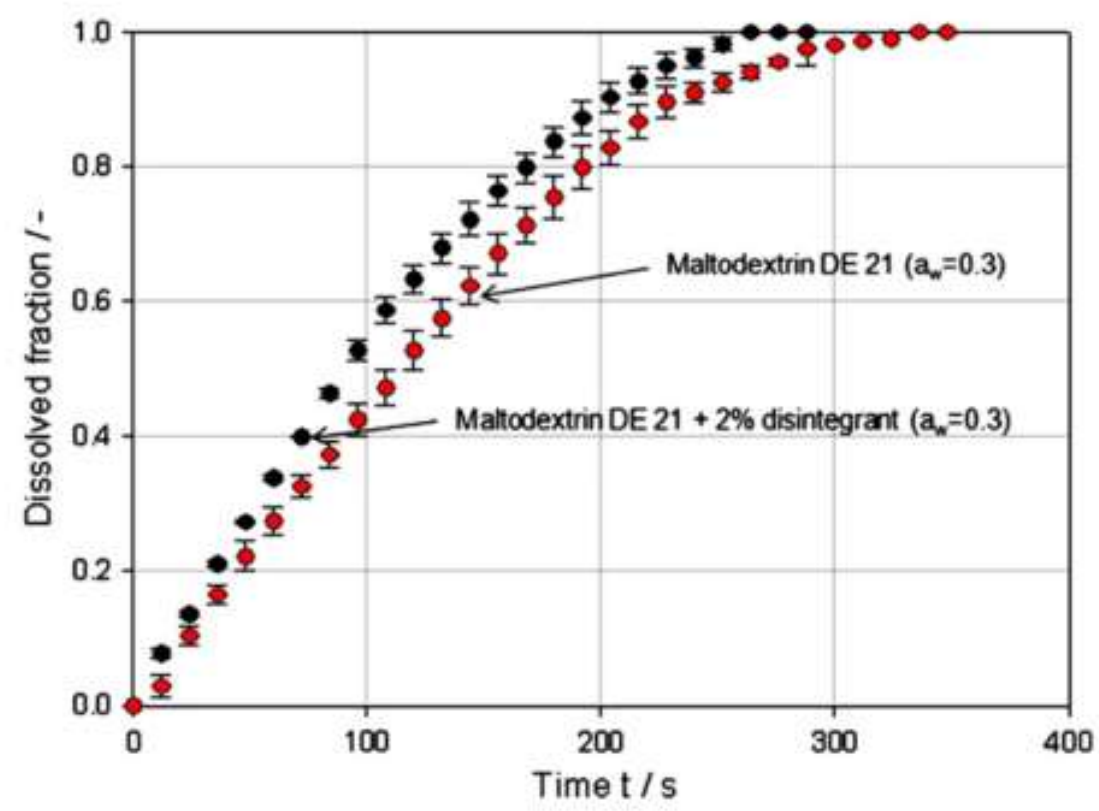

Figure 2.5 Dissolution profiles of tablets made of 100\% maltodextrin DE 21 and maltodextrin DE 21 containing $2 \%$ of disintegrant (croscarmellose sodium). (Mesnier et al., 2013)

The Flow Cell (see Figure 2.4) was used to capture images of the disintegration of the tablets which were then converted to binary images. A MATLAB program was used to identify the released particles and tablet dimensions. The variation of the tablet dimensions was observed, as well as the particle size distribution of the released particles, throughout the disintegration process.

Analysis of the evolution of tablet dimensions with respect to time showed that tablets containing $2 \%$ disintegrant decreased in both the tablet thickness and diameter faster than pure maltodextrin tablets (see Figure 2.6). The thickness of the tablet appeared to be decreasing at a faster rate at approximately $90 \mathrm{~s}$. It was hypothesised that this phenomenon was due to the increasing uptake of water into the tablet causing activation of the disintegrant located inside the tablet. It was noted that swelling did not occur during testing; maltodextrin does not swell when 
in contact with water, even with $2 \%$ disintegrant, as this is not a characteristic of this material. The change in tablet dimensions was generally a steady straight decline.
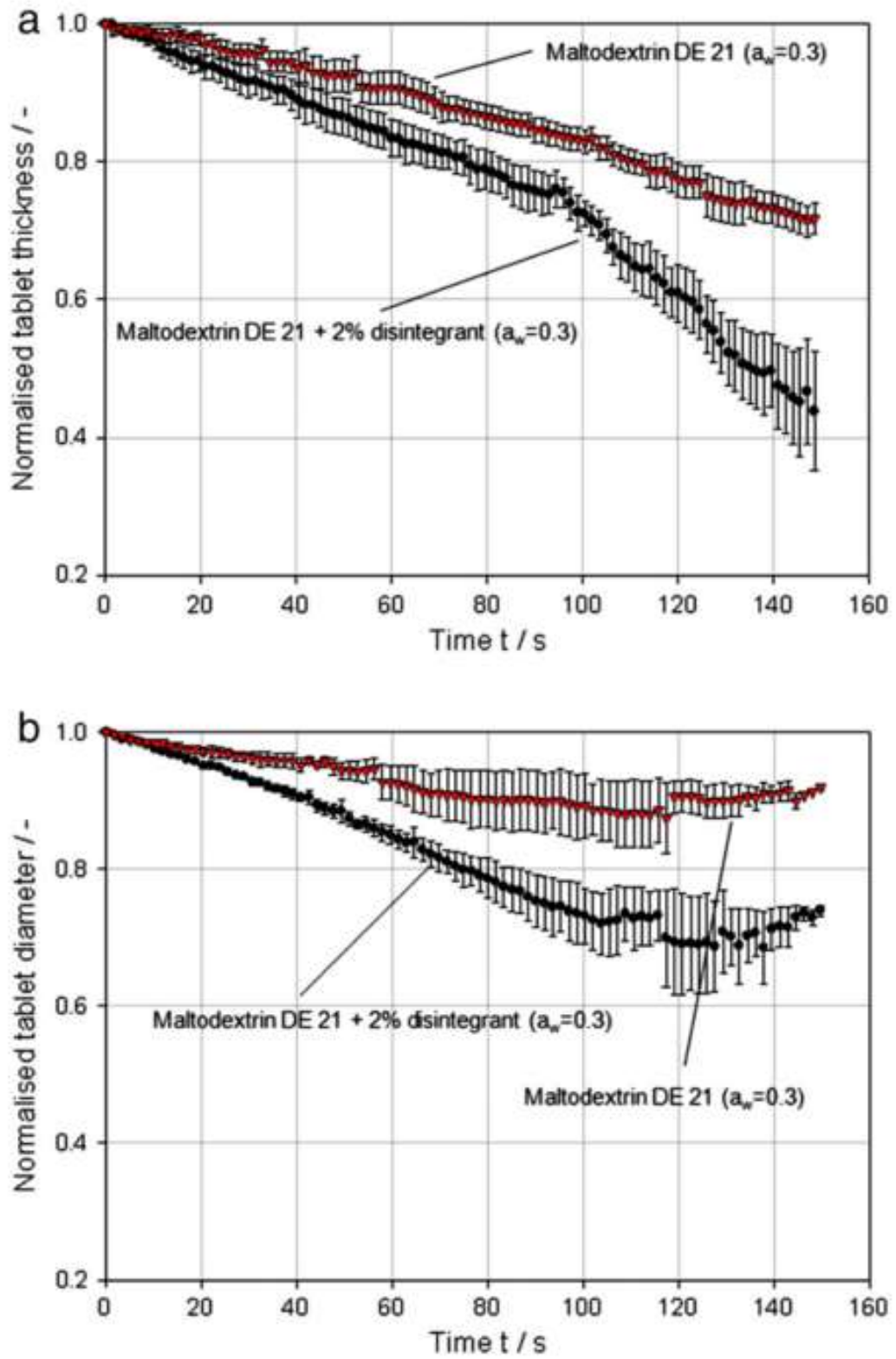

Figure 2.6 a) Variation of tablet thickness with time for tablets made of $100 \%$ maltodextrin DE 21 and maltodextrin DE 21 containing $2 \%$ of disintegrant. b) Variation of tablet diameter with time for tablets made of $100 \%$ maltodextrin DE 21 and maltodextrin DE 21 containing $2 \%$ of disintegrant. (Mesnier et al., 2013) 
The size distributions of the particles released showed that even without the presence of disintegrant, particles were still released from the tablets due to a phenomenon called "erosion". Erosion occurs due to the rupture of inter-particle bonding or polymer chains following contact with water. Tablets containing $2 \%$ disintegrant released a higher percentage of larger particles than pure maltodextrin tablets (see Figure 2.7). Maltodextrin primary particles were also tested to establish whether smaller sized particles were detectable by this analysis method. It was found that smaller particles could be detected, and therefore it was determined that in both tablet formulations aggregates rather than primary particles were released. This demonstrates that aggregates are formed during compression, where the bonding of primary particles occurs due to the tableting stress applied.

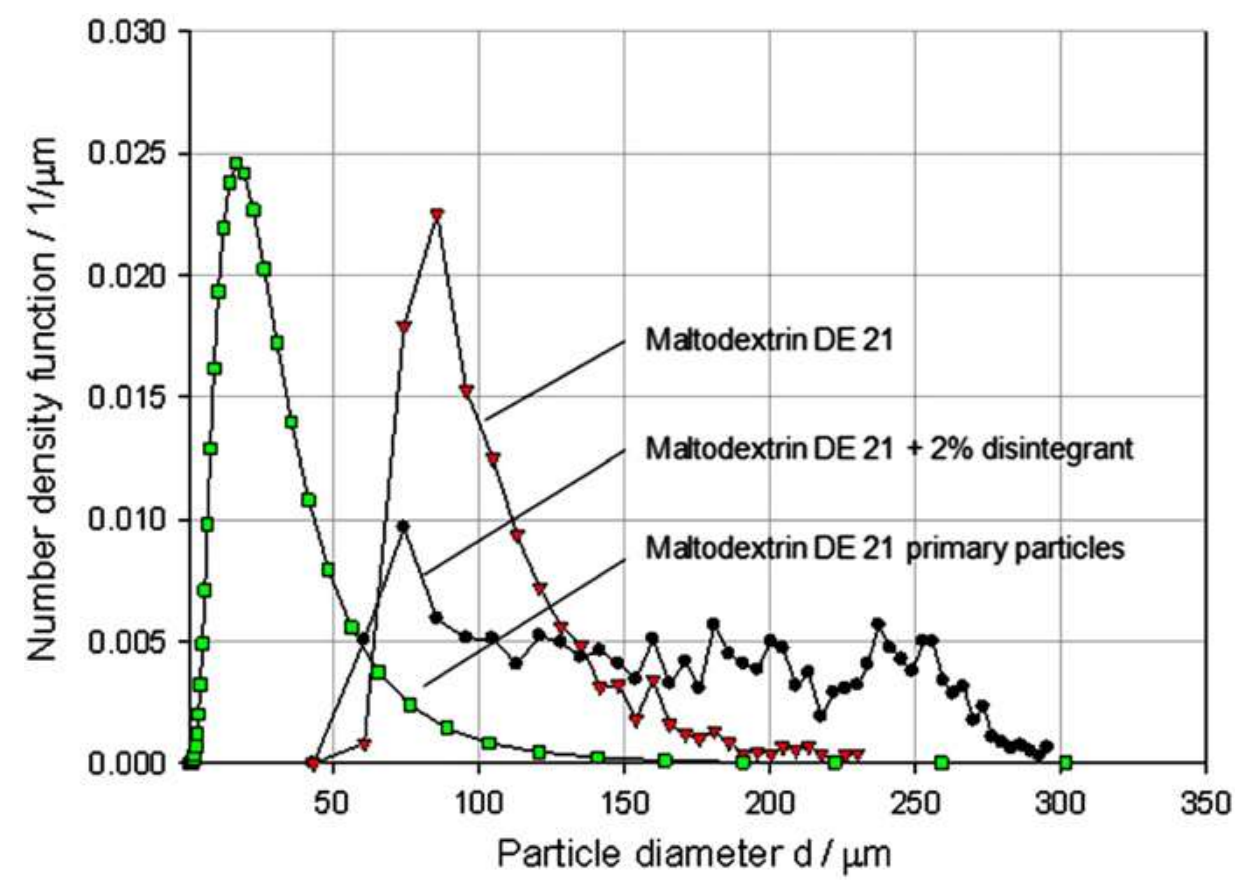

Figure 2.7 Density distribution of the particles liberated during the disintegration of tablets of maltodextrin DE21 and tablets of maltodextrin DE21 containing 2\% of disintegrants (croscarmellose sodium) obtained for the first 150s. (Mesnier et al., 2013) 
In the presence of disintegrant the total number of particles released and the mean particle size was found to be higher. This was rationalised to be due to the disintegrant causing fractures inside the tablet, allowing for larger particles to be liberated. The particle release data was grouped into 2 time sets rather than with time as the tablet thickness and diameter were previously presented. After $90 \mathrm{~s}$ of the disintegration, the number of particles released increased in size for the tablets containing disintegrant (see Figure 2.6 and Figure 2.8). This time period corresponded to the change in regime during the decrease of tablet thickness. Mesnier hypothesised three possible reasons for this to occur. Firstly, this phenomenon was attributed to the increasing diffusion coefficient of water as the penetration of water into the tablet increases. Secondly, it was suggested that a gradient of tablet density exists within a tablet, meaning that the top and bottom of a tablet are denser than the mid-section. Therefore, the mid-section of a tablet has a higher porosity, meaning that the penetration of water increases once this region is reached. This consequently causes a faster breakage of the tablet. Thirdly, the water may eventually be reaching and activating the disintegrant located inside the tablet. While the reasoning outlined need validation, the most important point to be made is that these nuanced variations in tablet disintegration would not have been detected if a conventional USP method was used. However, the rationale behind the differences in particle release sizes was not related to the tablet material properties. 


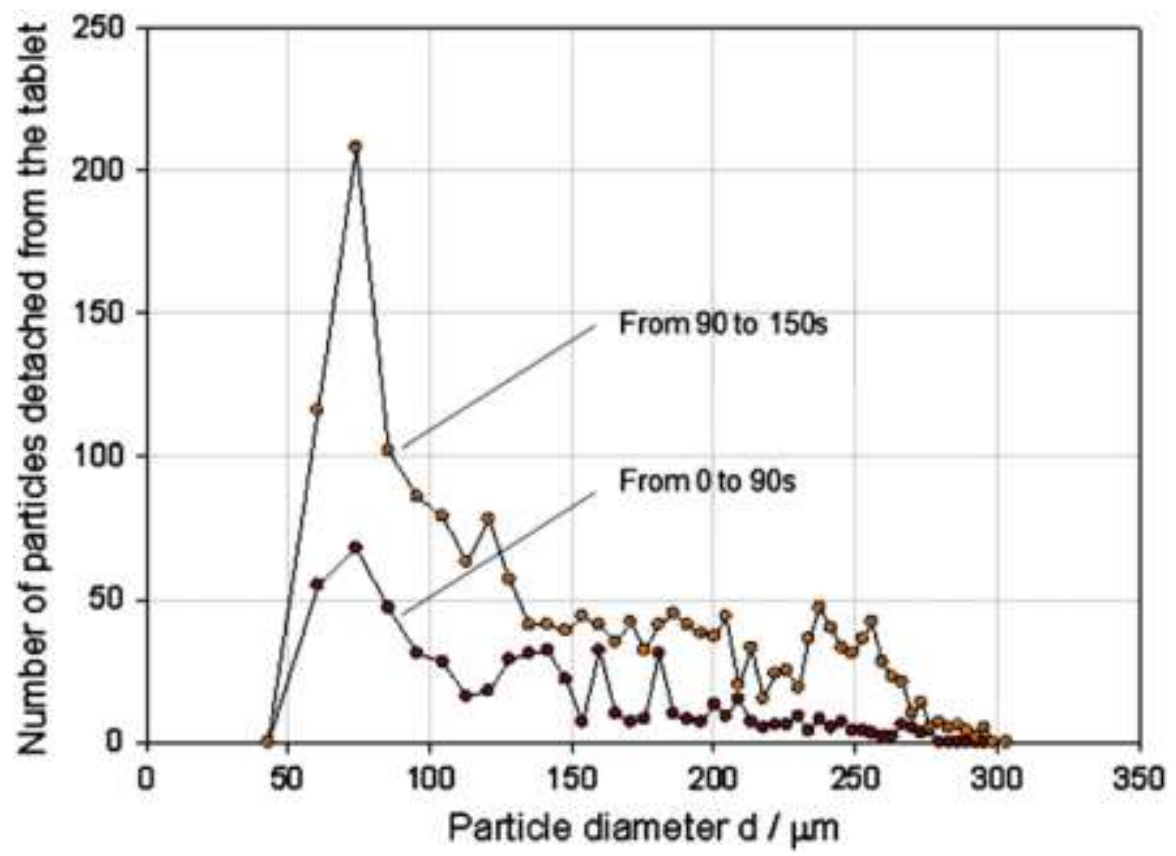

Figure 2.8 Variation of the number of the particles liberated during the disintegration of tablets of maltodextrin DE21 containing $2 \%$ of disintegrant (croscarmellose sodium) plotted for two different times: particles liberated from the start to 90s, and particle liberated after 90s to 150s. (Mesnier et al., 2013)

Wilson et al. used population balance modelling to link the dissolution process to disintegration by utilising an optical analysis method (Wilson et al., 2012). Tablet disintegration and dissolution was analysed using the standard USP method, and also by utilising a modified USP apparatus capable of monitoring particle release. Two batches of tablets (termed Tablet A and Tablet B for convenience) with differing physical properties were prepared from the same formulation using wet granulation (see Table 2.2). Tablet properties were altered through variation in the granulation and compression processes, resulting in tablets with differing crushing forces. Tablet A had a lower crushing force of $80 \mathrm{~N}$, whereas Tablet B had a value of $111 \mathrm{~N}$. 
Table 2.2 Tablet formulation.

\begin{tabular}{|cc|}
\hline Material & Percentage $w / w$ \\
\hline Drug substance & 5 \\
\hline Insoluble excipient & 15 \\
\hline Soluble excipient & 80 \\
\hline
\end{tabular}

Firstly, the disintegration time was measured as per the standard USP method and the time taken for the tablet fragments to fall through the mesh was identified as the disintegration time. For Tablet A a much shorter disintegration time was observed than for Tablet B. Standard dissolution testing was carried out in $\mathrm{pH} 1.4$ $\mathrm{NaCl} / \mathrm{HCl}$ dissolution medium with a paddle speed of $60 \mathrm{rpm}$. UV measurements at $343 \mathrm{~nm}$ were taken at various intervals to determine extent of drug dissolution, through comparison with a drug substance reference standard. Tablet A displayed a fast initial rate of release, then a slower phase which reached around $95 \%$ after 60 min. On the other hand, Tablet B presented a generally slower release rate which reached around $75 \%$ drug release after $60 \mathrm{~min}$. Thus a link between fast disintegration and fast dissolution for weaker tablets was established; however, no mechanistic insight was obtained through standard testing. 


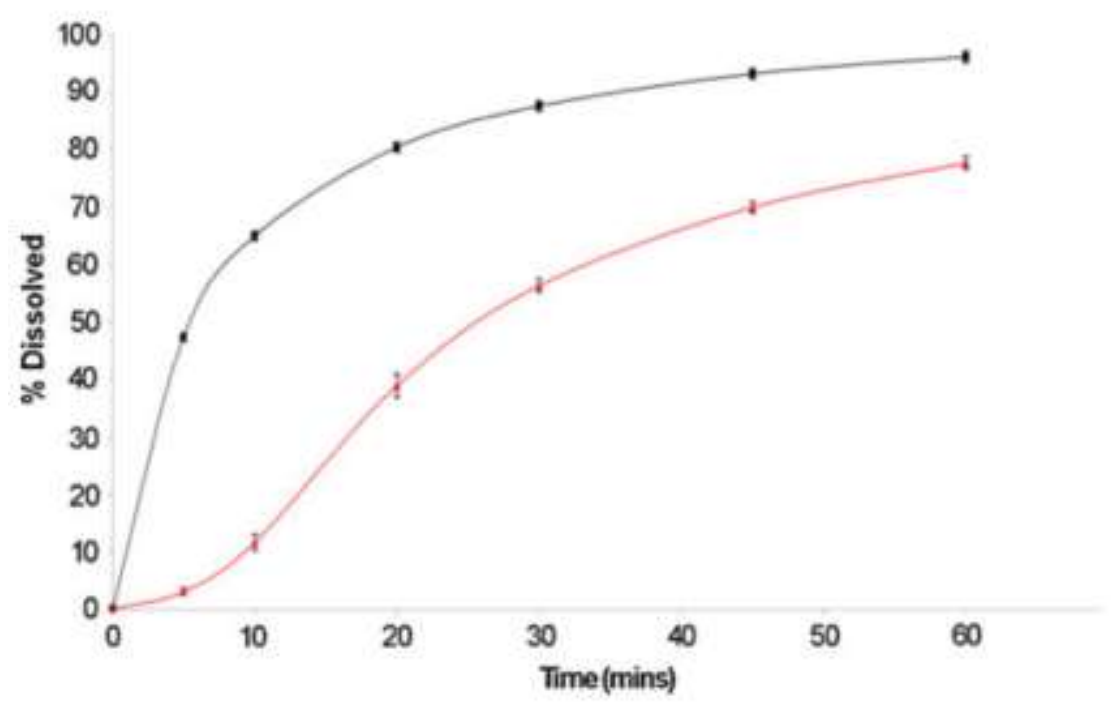

Figure 2.9 Dissolution profile obtained using UV analysis for Tablet A ( $(\mathbf{)})$ and Tablet B (A). (Wilson et al., 2012)

A standard USP apparatus, as described previously, was modified and connected directly to QICPIC equipment to investigate the number and size of particulates entrained in solution, as a function of time, during dissolution. Typically QICPIC equipment is used to measure particle size distributions of solid dry samples, however with the aid of the LIXELL unit attachment liquid dispersions are capable of being analysed. During testing the disintegration medium was pumped through the LIXELL system to analyse the particle release.

Firstly, it was observed that Tablet A released particles at a faster rate than Tablet B (see Figure 2.10). Tablet A reached its maximum number of suspended particles after $5 \mathrm{~min}$, whereas Tablet $\mathrm{B}$ reached its maximum after $30 \mathrm{~min}$. The number of entrained particles decreased gradually after the maximum is reached for both Tablet A and B; the decline is more pronounced for Tablet A than Tablet B. It was proposed that the trend for the decrease in number of entrained particles suggests that eventually the number of entrained particles will reach a constant 
value, which could correspond to the presence of $15 \% \mathrm{w} / \mathrm{w}$ insoluble excipients in the formulation.

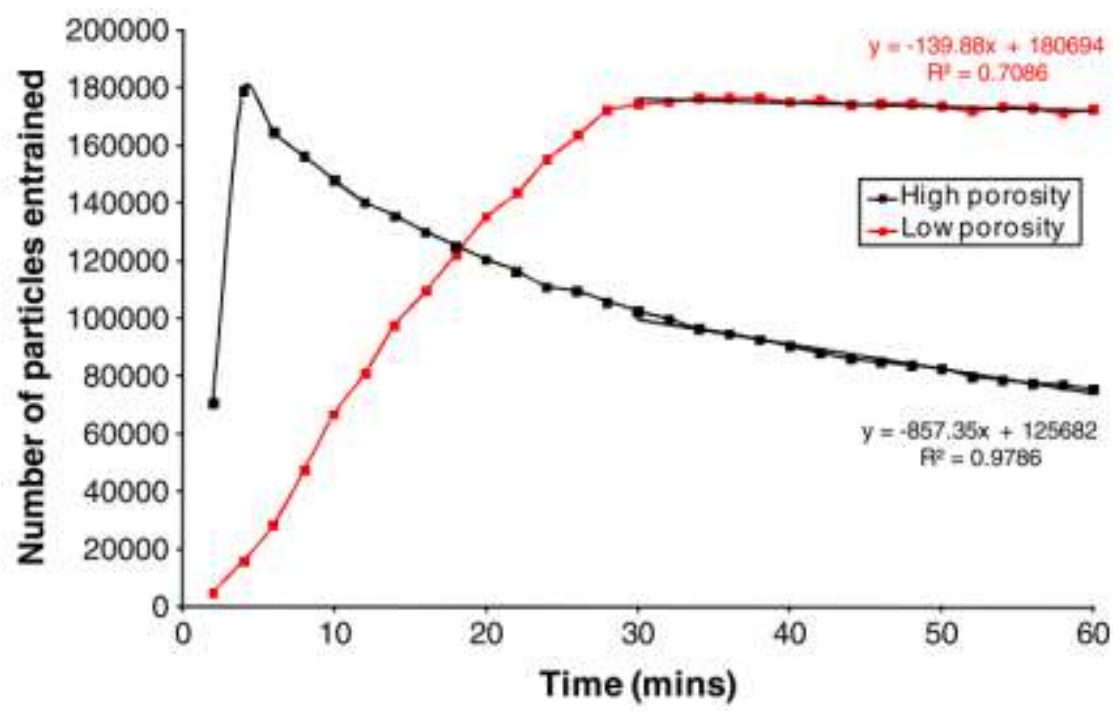

Figure 2.10 Number of particles released with time for Tablet $A(\square)$ and Tablet $B(\Delta)$. (Wilson et al., 2012)

However, it seemed that Tablet A and B were progressing towards different final entrained particle values. The cause of the difference was deemed to be unclear; whether this was due to the generally slower rate of dissolution of Tablet B or a difference in the degree of insoluble particle agglomeration was not confirmed. Nevertheless, it was seen that a strong link between the rate of change in entrained particles and the rate of dissolution exists.

The mass weighted size distributions (see Figure 2.11) were observed and similar size ranges were detected for both Tablet A and B. However, the ratios of the various size ranges varied during dissolution. In the case of Tablet $A$ the number of smaller particles decreased with time, whereas the number of larger particles remained fairly constant throughout the dissolution. On the other hand Tablet B showed that the number of particles remained relatively constant throughout the 
dissolution time. The number of particles in each respective size range corresponds to the soluble and insoluble excipients present in the formulation; therefore, the decreasing number of particles in the smaller size range was attributed to the dissolution of the dispersed drug particles. The volume weighted $\mathrm{D}_{50}$ values as a function of time remained relatively constant for Tablet A. Tablet B however presented a reduction in the $D_{50}$ value with time. It was suggested that this could represent a secondary step during dissolution where Tablet B undergoes a reduction in size.
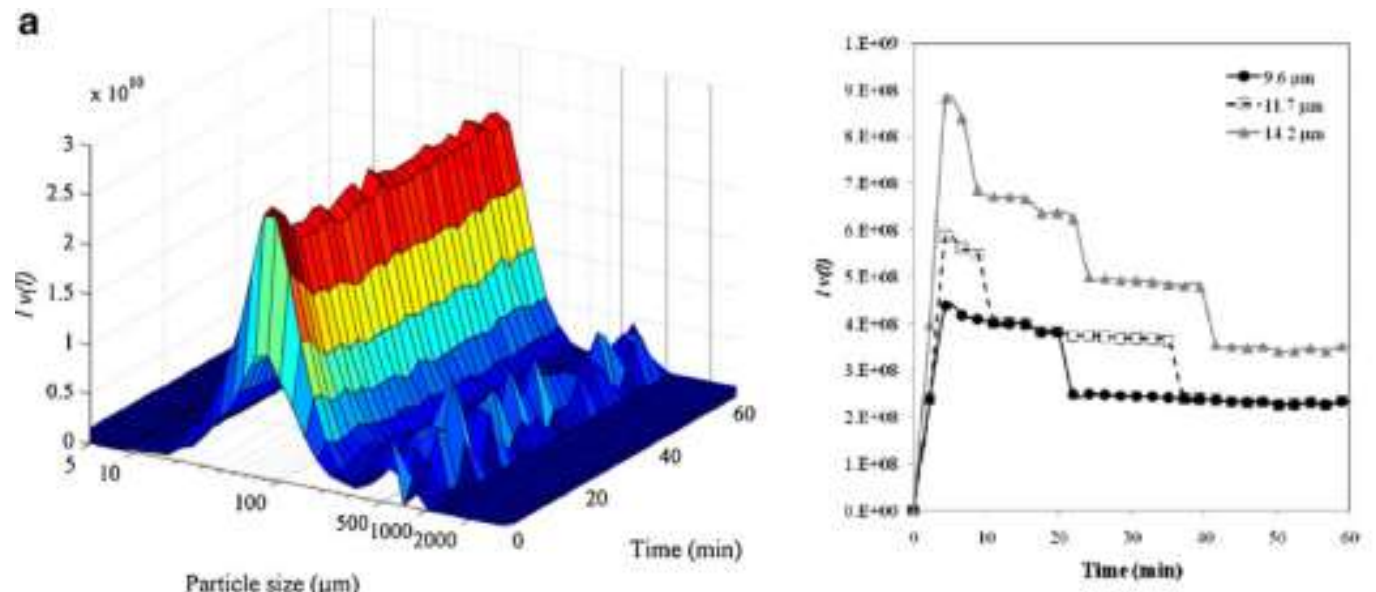

b
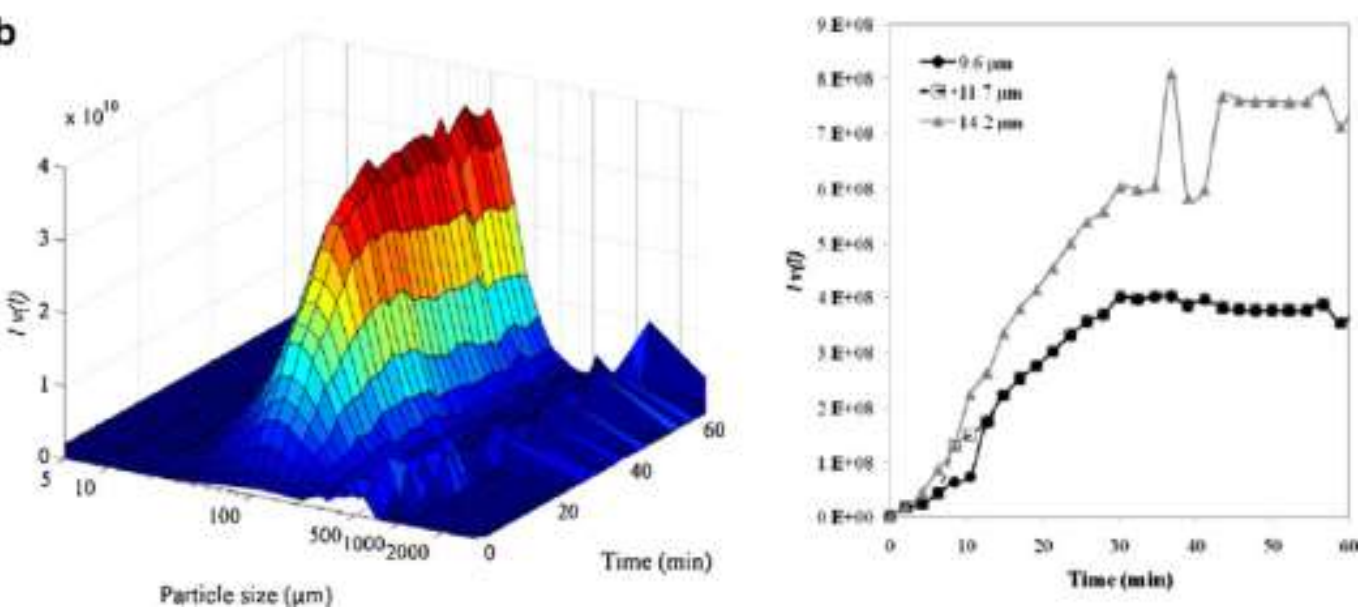

Figure 2.11 Mass weighted particle size distributions of tablet A (top images) and tablet $B$ (bottom image). Left side images show the total distribution; right side images show three small size classes in the approximate location of the drug substance. (Wilson et al., 2012) 
To correlate the different tablet dissolution profiles and the rate of particle release for Tablets $\mathrm{A}$ and $\mathrm{B}$, the rate and extent of dissolution was considered. It was found that Tablet $\mathrm{A}$, which has a faster rate of dissolution, tends to have more particles entrained in solution. It can be assumed that the rate of drug dissolution is proportional to the number of particles entrained and the rate of particle release, as a higher number of particles means a larger particle surface area is available for dissolution to occur.

While this method has been proven to provide additional knowledge during disintegration analysis, the methodology contains some flaws which may influence the results obtained. Due to the nature of the modified USP equipment, the disintegration media is required to be pumped to the QICPIC viewing window for the particle imaging analysis to occur. Therefore, it must be considered that the entrained particles are subjected to further mechanical stress in addition to the existing agitation of the USP apparatus. This suggests that the entrained particles may undergo breakage, meaning that when they are eventually measured, the size observed would be significantly misrepresented.

\subsubsection{Dissolution of tablets}

Dissolution of tablets is complex and involves many processes occurring simultaneously. Dissolution can be defined as the process of solid soluble material transitioning into a solute state. Tablet disintegration is the breakage of the tablet into smaller pieces following contact with the liquid media, thus increasing the surface area available for dissolution to occur (Desai et al., 2016). Disintegrants are therefore added to pharmaceutical tablets to speed up the dissolution process, for example in fast release tablets (Pabari and Ramtoola, 2012). In literature, it has 
previously been suggested that dissolution of a soluble material is not limited by disintegration (see Figure 2.12); dissolution is also possible from the solid dosage form. However, disintegration offers additional routes for dissolution to occur (Ibrahim and Sallam, 1993, Wagner, 1969). Disintegration can be thought of as an integral process for the dissolution of tablets; both processes coincide with and affect each other. However, disintegration and dissolution are rarely studied as mutual processes.

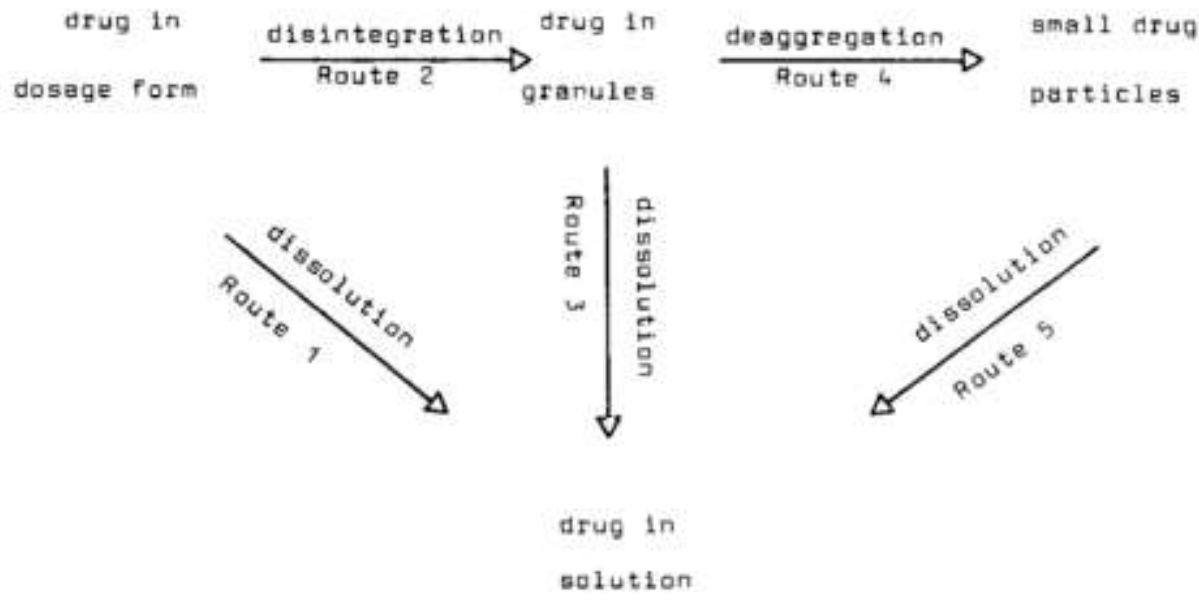

Figure 2.12 Various proposed routes for dissolution to occur. (Ibrahim and Sallam, 1993)

The time taken for a tablet to disintegrate is measured using a standardised pharmacopoeial testing apparatus. Measurement of the dissolution of the active pharmaceutical ingredient (API) dissolution time is carried out via chemical analysis (Katzhendler et al., 1997). The standard United States Pharmacopeial (USP) methods used to analyse dissolution are outlined in Section 2.2.

Current methods used in industry to analyse tablet dissolution have been challenged through research. Röst and Quist studied effect of varying the stirring conditions, a fundamental component of the USP equipment, on the dissolution of 
United States Pharmacopoeial Convention (USP) prednisone calibrator tablets (Röst and Quist, 2003). For this research an automated USP-II dissolution test apparatus was fitted with a special paddle-propeller. Dissolution tests were carried out whilst altering the stirring conditions through variation of the paddle to either a pulling or pushing propeller by changing the angle of the paddle blades. UV analysis was utilised to determine the API release. The fastest dissolution was obtained with the paddle-propeller blade angle at around $+30^{\circ}$, providing a pushing, downward flow at the centre of the vessel. This contradicts previous research which suggested a paddle at a blade angle of $0^{\circ}$ allowed the best stirring. This research suggests that the USP dissolution analysis methods are not as accurate as intended. Measurement of the exact dissolution time, i.e. the rate of the API release, can vary depending on the set-up of the apparatus.

Various methods have been explored in research to investigate dissolution in a manner which allows for additional information to be gained when compared to the standard USP testing methods. Delalonde stated that the kinetics of dissolution is dependent on various phenomena such as mass transfer, fluid phase filtration and volume variation (Delalonde and Ruiz, 2008). In an attempt to understand the different processes involved in dissolution, a hydro-textural approach was integrated into the existing European Pharmacopoeia standard tablet dissolution analysis. A discontinuous and continuous analysis of tablet dissolution was performed on the theophylline tablets which were produced at varying forces. 
a

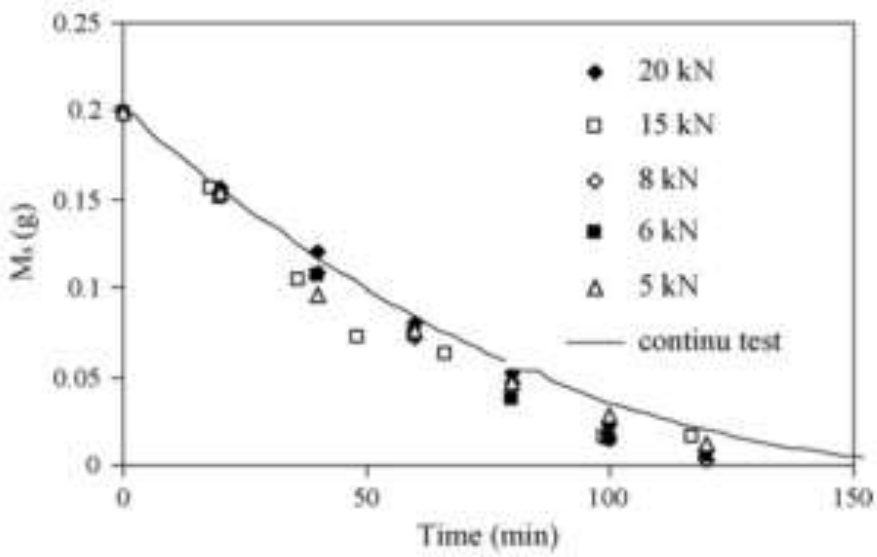

b
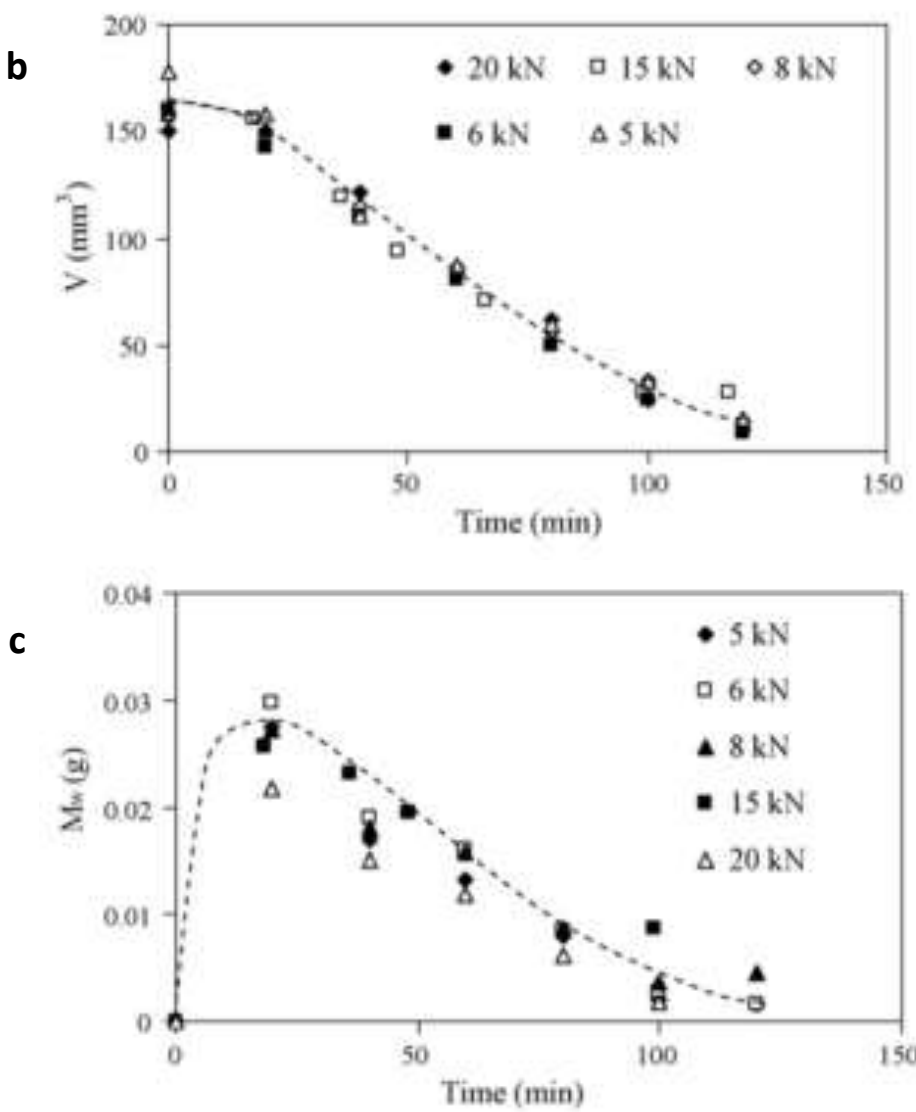

Figure 2.13 Evolution of a) $M_{s}$, b) $V$ and c) $M_{w}$ with time for theophylline tablets produced using varying tableting forces. (Delalonde and Ruiz, 2008)

The tablets were characterised beforehand by their initial mass and volume ( $\mathrm{M}_{0}$ and $\mathrm{V}_{0}$, respectively) for later calculations (Delalonde and Ruiz, 2008). As seen in Figure 2.13 it was found that the evolution of the remaining dry mass $\left(\mathrm{M}_{\mathrm{s}}\right)$ is independent of compacting force, and both methods were in close agreement. The 
evolution of tablet volume (V) vs. time suggested that whilst compaction force influences dissolution, the effects are limited to the outer tablet surface. The evolution of penetrated water mass $\left(\mathrm{M}_{\mathrm{w}}\right)$ as a function of time was studied. The penetration of water was proposed to occur in 3 stages. The first stage was explained as water penetration occurring through capillary action until equilibrium is reached between the air and water pressure inside the tablet pores. The second stage corresponded to water enclosing the tablet and the porous structures. This causes air inside the tablet to become entrapped, limiting the amount of water which can penetrate the tablet until the air escapes. The final stage was related to the gradual dissolution occurring at the tablet periphery which eventually opens porous pathways for air outflow, leading to complete dissolution. A higher tablet compaction force led to a slower penetration of water into the tablet. Investigation of the variation in the degree of tablet saturation as a function of time also revealed that the tablet samples are never fully saturated, and the cores remain dry until near-complete dissolution. From this method, the disintegration of the tablet was also considered in terms of the change in tablet mass and volume. However, the reasoning behind any differences in the changing tablet dimensions is speculative. A visual monitoring system would provide further evidence to support the reasoning given.

The dissolution kinetics of theophylline granules and dry tablets were compared using standard dissolution test apparatus (Delalonde and Ruiz, 2008). At both 50 and $100 \mathrm{rpm}$ it was found that granules dissolve faster than the tablets, due to the increased surface area in contact with the dissolution media. For both granules and tablets dissolution occurred faster at $100 \mathrm{rpm}$, which suggests the 
limiting factor is the rate of diffusion from the boundary layer and is not related to molecular diffusion rate.

Near-infrared (NIR) spectroscopy was employed to investigate the dissolution process in pharmaceutical tablets at a molecular level (Hattori and Otsuka, 2011). Tablets were prepared containing either $\alpha$-lactose monohydrate (La) or trehalose (Tr) fillers, magnesium stearate $(\mathrm{MgSt})$ and riboflavin $(\mathrm{RF})$ at varying porosity $(\mathrm{H}-$ high, M-medium, L-low). La-H, La-M, La-L and Tr-M tablets were characterised by their mechanical strength, disintegration time and dissolution profiles.

UV spectroscopy dissolution profiles indicated that the porosity of the tablets influenced the speed of dissolution, with the most porous La-H tablets dissolving at the quickest rate. However, it was found that the initial dissolution was governed by tablet disintegration; water penetration profiles of the tablets were obtained to investigate further. The Lucas-Washburn theory was utilised to explain the behaviour, which is summarised by Equation 2.1.

$$
W^{2}=\frac{\rho_{\text {liq }}{ }^{2} A_{\text {ave }}{ }^{2} \gamma \cos \theta}{2 \eta S} \frac{\varepsilon^{3}}{1-\varepsilon}
$$

Equation 2.1

where $\mathrm{W}$ is the mass of penetrated liquid; pliq and $\eta$ are the density and viscosity of the liquid, respectively; $\gamma$ and $\theta$ are the surface tension and the contact angle between the liquid and particle layer, respectively; Aave, $\varepsilon$ and $S$ are the averaged sectional area, porosity and the specific surface of the particle layer, respectively.

$\mathrm{W}^{2}$ is linearly proportional to $\mathrm{t}$ (Equation 2.1 ) for that reason straight lines were fitted to $\mathrm{W}^{2}$ vs. $\mathrm{t}$ for all sample tablets. From Equation 2.1 it can be seen that porosity $(\varepsilon)$ directly contributes to the penetration rate, which was confirmed by the 
comparable water penetration profiles of La-M and $\operatorname{Tr}-\mathrm{M}$ tablets, whereas their respective dissolution profiles were dissimilar.

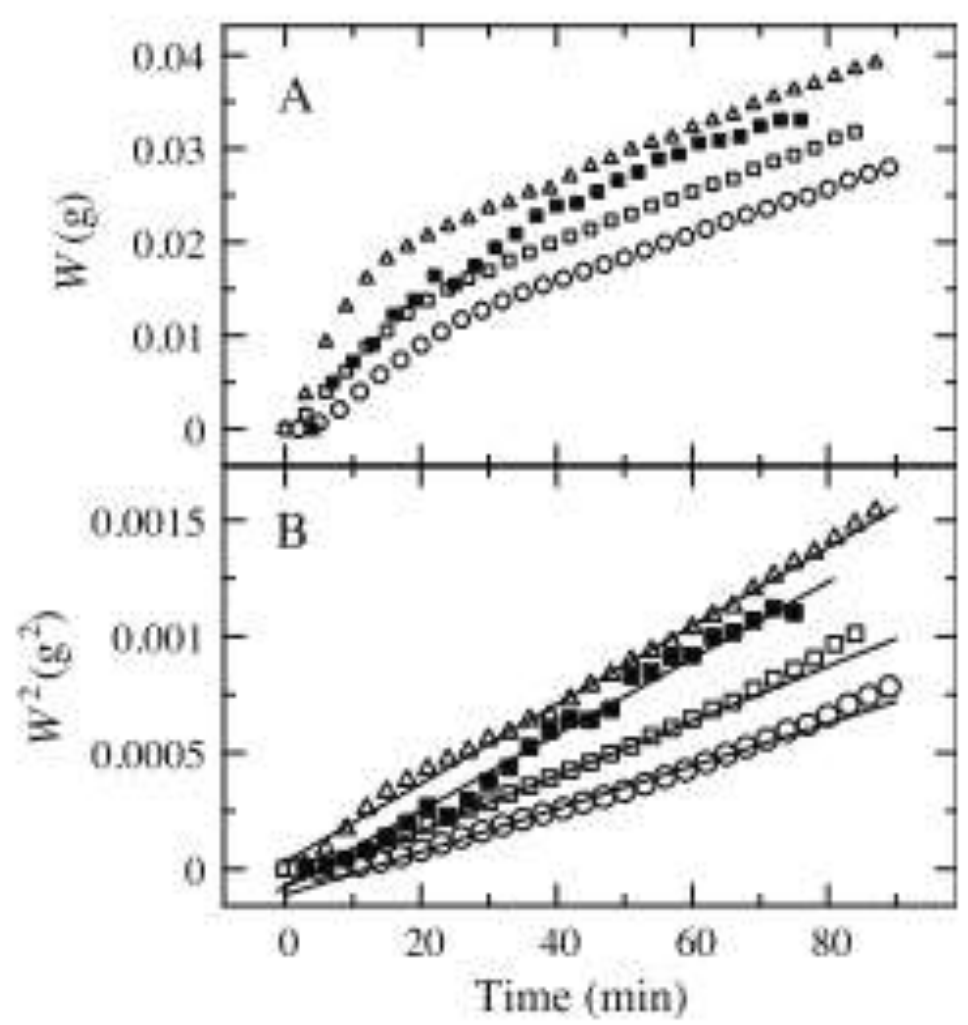

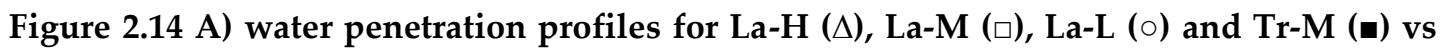
time and B) W2 vs time. Solid lines are fit to linear lines on the basis of the LucasWashburn theory. (Hattori and Otsuka, 2011)

The interactions between water molecules and the tablet excipients were studied by utilising NIR spectroscopy and the perturbation-correlation movingwindow two-dimensional correlation spectroscopy (PCMW-2DCS) technique (Hattori and Otsuka, 2011). Spectral changes upon water penetration in the tablet samples suggested that dissolution was occurring through two processes; (i) interaction between water and tablet disaccharide through water penetration, 
depending on disaccharide hydration number; (ii) saturated disaccharide leading dissolution and diffusion in water, depending on tablet porosity.

Overall, it was confirmed that tablet porosity and the rate of water penetration both play a role in the tablet dissolution by using NIR spectroscopic measurements. While this is a viable on-line method for the analysis of tablets, the tablet dissolution is only considered in relation to the whole tablet swelling. The particle release, which is a vital mechanism which aids tablet disintegration and consequently dissolution, is not deliberated.

Magnetic resonance imaging (MRI) is generally used in the medical field for clinical diagnoses; however, recent advances have enabled utilisation of laboratoryscale MRI equipment in investigating the dissolution process of pharmaceutical tablets (Nott, 2010). A low-field MRI instrument integrated with a MRI compatible USP-4 dissolution apparatus was used to image the various stages during the dissolution of USP standard gel matrix tablets. The dissolution apparatus contained glass beads to ensure laminar flow throughout and unrestricted swelling of the tablets. A UV spectrometer was also fitted to the equipment to simultaneously monitor the concentration of drug release during dissolution. By varying the contour intensities of the MR images obtained during dissolution it was possible to differentiate between, and calculate the area of the whole tablet and non-hydrated core at each stage. This provided useful information as to the hydration process during dissolution and can be used to investigate the effects of hydration on overall dissolution rates.

From the methods previously mentioned (Hattori and Otsuka, 2011, Nott, 2010, Röst and Quist, 2003), it was noticed that the preferred dissolution analysis technique used was UV spectroscopy. UV spectroscopy is an unreliable technique if 
multiple soluble components exist within the tablet being analysed, as the respective spectra may overlap and therefore the calculated concentrations in solution may be inaccurate. However, in terms of the effect of tablet porosity, it has been generally found that more porous tablets propagate faster dissolution and disintegration.

Coombes et al. used ${ }^{1} \mathrm{H}$ NMR to monitor dissolution of commercially available cold medicine tablets containing a mixture of 4 difference compounds (Coombes et al., 2014). NMR is typically used as a characterisation technique to determine the chemical structure of a compound. Due to the accuracy of the method it was possible to determine the increase in concentration of each individual component with time. While this is advantageous due to the accuracy of the measurement compared to technique such as UV, it is an off-line technique. This means that the concentrations in solution are possibly not an accurate representation of the dissolved percentage at the time that the sample was taken.

${ }^{1} \mathrm{H}$ NMR was also used in conjunction with MRI to monitor tablet dissolution and visually monitor tablet disintegration (Wren et al., 2017). Tablets were produced using mannitol and sodium starch glycolate which was granulated. Wet granulation parameters and the granule size fractions were varied. It was found that tablets which were produced using the highest granulation intensity and larger granules decreased in tablet volume at the slowest rate and released mannitol at the slowest rate. 


\section{Chapter 3. Roller Compaction and Tableting}

\subsection{Introduction}

This Chapter will explore the effect of the stresses during roller compaction (RC) granulation and tableting on ribbon and tablet properties. Ribbon porosity affects the consequent tablet porosity, which is one of the main properties which affect the tablet dissolution and disintegration. It has been reported in literature that porous tablets will disintegrate quickly due to the rapid uptake of water through the tablets pores (Pitt and Sinka, 2007). The tablet strength and porosity are fundamentally determined by the roller compaction and tableting stresses used during production. Careful manipulation of these parameters can lead to the desired properties manifesting in the consequent tablets.

When a tablet is produced using roller compaction granulation, the primary powder is subjected to two key stresses; the first stress is during granulation between the rollers and the second stress is during tableting. Compression of a material through the application of stress leads to a decrease in the material volume and changes in the mechanical strength of said material due to the formation of particle-particle bonding. Therefore, when materials undergo granulation and tableting, the tablet properties, such as tensile strength and porosity, are affected by these stress applied at each stage. The application of stress can be both beneficial 
and detrimental to the material properties within a tablet, as there is a balance between making the material stronger or weaker as a whole with more stress applied (Johansson et al., 1995, van der Voort Maarschalk et al., 1996, Zea et al., 2013).

In literature, the tablet tensile strength and porosity are key tablet properties which are examined to quantify tablet functionality. Some basic terms which are used to describe these properties are as follows. The tensile strength and porosity of tablet is defined as the "compactibility". This tensile strength of a tablet in terms of the tableting compaction pressure used is termed as the "tabletability" of a material. The tablet porosity in terms of the tableting compaction pressure used is termed as the "compressibility".

In this chapter the impact of the two main stresses applied during tablet production, roller compaction and tableting, will be investigated. The effect of the $\mathrm{RC}$ pressure and tableting force on the tablet compactibility will be examined by measurement of the tensile strength and porosity. X-ray tomography, which is capable of viewing the internal tablet structure (Busignies et al., 2006), was also used to elucidate the effect of these stresses on the internal structure of the tablet.

\subsection{Materials}

In Chapter 3 - Chapter 5 placebo mixtures will be analysed to determine the effect of granulating and tableting a ductile and a brittle material. The excipient materials were used as received, unless otherwise stated. 


\subsubsection{Mannitol (Pearlitol 200SD)}

Mannitol Pearlitol 200SD from Roquette (Lestrem, France) was studied as it is a soluble and brittle filler material. Mannitol is formed by the catalytic or electrolytic reduction of monosaccharides such as mannose and glucose. In this case the mannitol was formed by spray drying, and is a white, odourless powder. Mannitol is commonly used in the pharmaceutical and food industry due its sweet taste and "mouth-feel" (Rowe et al., 2009). Figure 3.1 shows the slightly spherical shape of spray dried mannitol powder from SEM images. It is capable of being directly compressed or granulated by either wet or dry granulation.
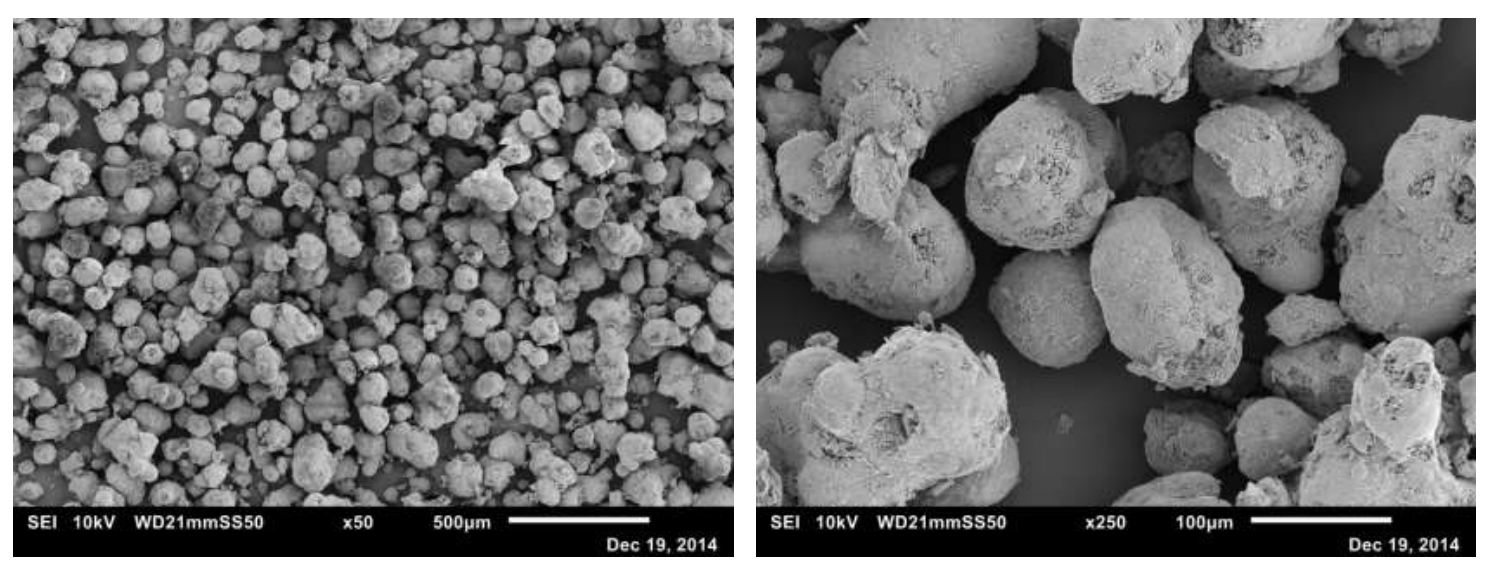

Figure 3.1 SEM images of Mannitol Pearlitol 200SD at x50 and x250 magnification.

\subsubsection{Microcrystalline cellulose (Avicel PH-101)}

Microcrystalline cellulose (MCC) Avicel PH101 from Sigma Aldrich (St. Louis, USA) was also studied as filler. MCC is an insoluble and ductile material. It is a purified, partially depolymerised cellulose which occurs as a white, odourless, crystalline powder (Rowe et al., 2009). It well suited for direct compression and wet or dry granulation. MCC is able to undergo significant plastic deformation and form strong compacts. Therefore, it is widely used in the pharmaceutical industry as a 
binder and filler; in some cases it exhibits disintegrant behaviour as it readily absorbs and swells when in contact with water. SEM images of MCC are shown in and Figure 3.2 where the elongated crystalline structure is clear.
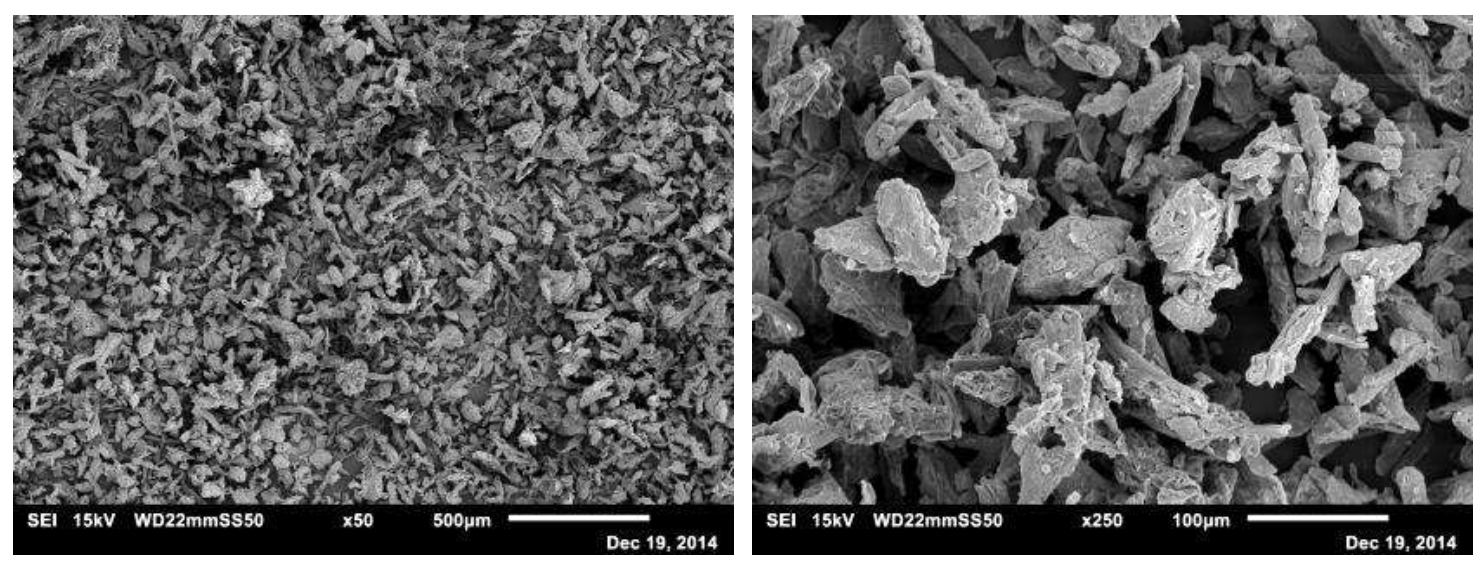

Figure 3.2 SEM images of MCC Avicel PH101 at x50 and x250 magnification.

\subsubsection{Magnesium stearate (Mallinkrodt)}

Magnesium stearate Mallinkrodt (St. Louis, USA) was used as a lubricant during granulation and tableting. It is a fine, white, greasy powder which is highly hydrophobic (Rowe et al., 2009)

\subsubsection{Filler particle size and solubility}

The particle size values and solubility data are shown in Table 3.1. Mannitol generally has a larger primary powder size.

Table 3.1 $\mathrm{D}_{10}, \mathrm{D}_{50}$ and $\mathrm{D}_{90}$ values and the solubility data for the filler materials which were examined (Rowe et al., 2009).

\begin{tabular}{|ccccc|}
\hline Material & $\mathbf{D}_{10}$ & $\mathbf{D}_{50}$ & $\mathbf{D}_{90}$ & $\begin{array}{c}\text { Solubility in water } \\
\text { (part/part) }\end{array}$ \\
\hline Mannitol (200SD) & 100 & 173 & 250 & 1 in 5.5 \\
\hline MCC (PH101) & $14-30$ & $40-75$ & $77-156$ & {$[$ insoluble] } \\
\hline
\end{tabular}




\subsubsection{Binary placebo mixtures}

The placebo binary mixtures which will be analysed in the following Chapters are outlined in Table 3.2. The percentages of the fillers mannitol and MCC are varied, as well as the processing parameters.

Table 3.2 Formulations and processing conditions used to produce binary mixture granules with abbreviations.

\begin{tabular}{|ccc|}
\hline Abbreviations & Mannitol (\%) & MCC (\%) \\
\hline Man100:MCC0 & 100 & 0 \\
\hline Man75:MCC25 & 75 & 25 \\
\hline Man50:MCC50 & 50 & 50 \\
\hline Man25:MCC75 & 25 & 75 \\
\hline Man0:MCC100 & 0 & 100 \\
\hline
\end{tabular}

\subsection{Methods}

\subsubsection{Scanning electron microscopy (SEM)}

A CamScan S2 scanning electron microscope (CamScan, UK) was used to obtain electron micrographs of primary powder. The equipment operates under vacuum conditions at $10 \mathrm{kV}$. The tablets were pre-coated with gold to improve the conductivity of the material using an Emscope SC 500A sputter coater. 


\subsubsection{Roller compaction}

Before granulation, the powders were pre-mixed using a Roto Junior Zanchetta \& C (Lucca, Italy) mixer at $100 \mathrm{rpm}$ for $5 \mathrm{~min}$. The mixtures were then conditioned in a Binder KMF240 Climactic humidity chamber (Tuttlingen, Germany) at $25{ }^{\circ} \mathrm{C}$ and $40 \%$ relative humidity for 3 days to ensure constant moisture content within the powders. The formulations that were analysed are outlined in the respective Chapters. The main two materials which were used are mannitol and microcrystalline cellulose (MCC). The moisture content of mannitol increases drastically at $>75 \%$ relative humidity and MCC is hygroscopic and therefore the conditioning is required so that the powder properties remain constant for all formulations (Rowe et al., 2009).

All ribbons and granules were produced using an Alexanderwerk WP 120 PHARMA (Remscheid, Germany) roller compactor. A diagram of the roller compactor equipment which was used in this research, with horizontally mounted rollers, is illustrated in Figure 3.3.

For the roller compactor used in this research, the powder is introduced through the hopper and into the screw feeder. This conveys the material through the two counter current rollers which apply stress to densify the material and produces a compacted ribbon. The ribbon compact can then be crushed, for example by a crushing mill, which produces granules with improved properties such as flowability and homogeneity (i.e. less segregation of component materials within the formulation). The granules can then be tabletted into solid oral dosage forms. 


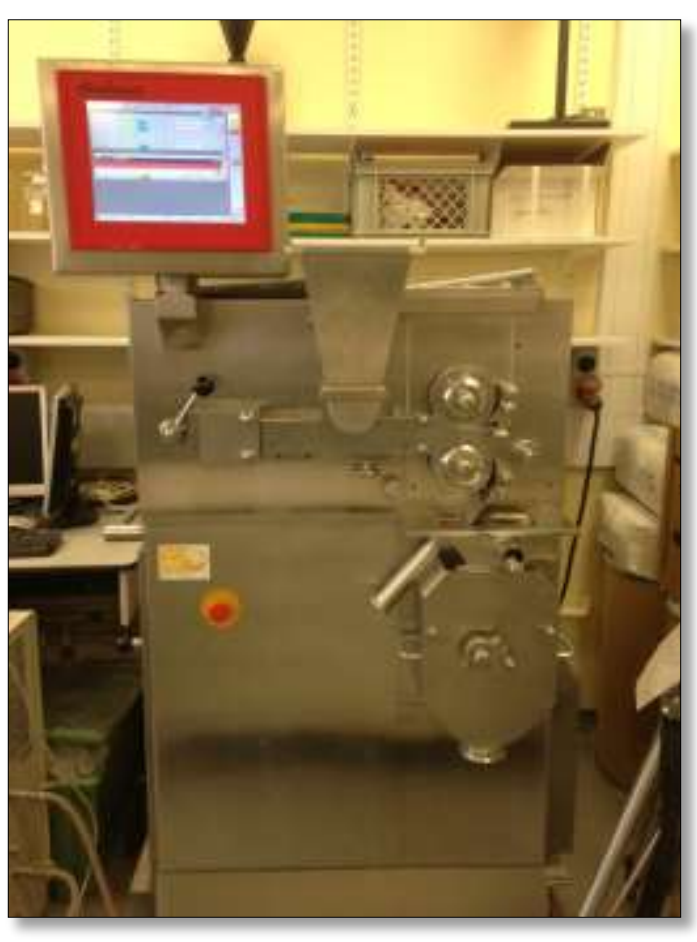

\section{Powder}

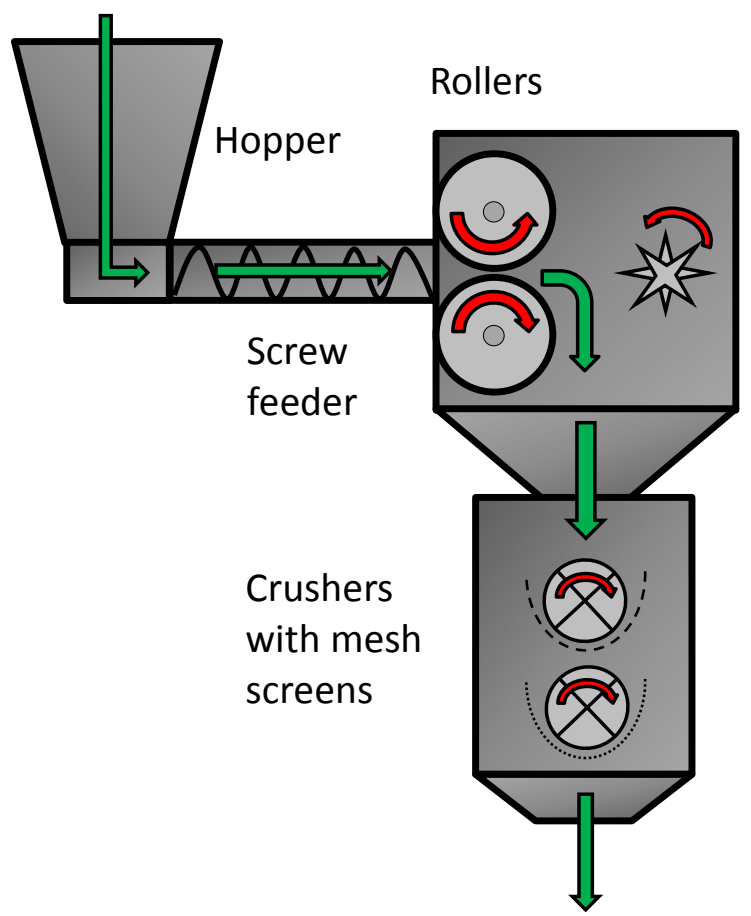

Granules

Figure 3.3 Photograph and schematic of the roller compactor used in this research.

In this research, the conditioned powder mixtures were roller compacted using a screw speed of $19 \mathrm{rpm}$ and roller speed of $6 \mathrm{rpm}$. These settings were used as they were suitable for the materials that were studied in this research, meaning that the material was able to be carried through the equipment with no blockages. The roller dimensions were as follows; the roller width used was $0.04 \mathrm{~m}$, the roller diameter was $0.12 \mathrm{~m}$ and the roller gap used was $0.003 \mathrm{~m}$. Two meshes were used to crush ribbons into granules, where the larger mesh had a screen size of $3.25 \mathrm{~mm}$ and the smaller mesh had a screen size of $1.5 \mathrm{~mm}$. Magnesium stearate was used as an external lubricant during roller compaction to prevent sticking.

In this research the stress applied to a material during the journey of producing a tablet is a key parameter that will be examined. The first stress applied 
to a material is during $\mathrm{RC}$, as pressure is applied between the rollers to compact the powder into ribbons. Therefore, two RC pressures were chosen, 20 and 100 bar, to ensure that the ribbons which were produced would exhibit a range of properties which can be examined.

\subsubsection{Ring shear tester}

A RST-XS Ring Shear Tester (Dietmar-Schulze, Germany) was used to determine the effective angle of internal friction $(\delta \mathrm{E})$ and the angle of wall friction $(\varphi w)$. The measurements were carried out using $10 \mathrm{kPa}$ normal load at preshear.

\subsubsection{Johanson Model}

According to the Johanson model, the pressure gradients experienced by the powder material during roller compaction can be determined by inputting the relevant powder properties. The model can be used to link the powder properties to the processing parameters used, and furthermore be used to relate the hydraulic pressure applied by the rolls to the peak pressure applied to the material as stress.

Equation 3.1 and Equation 3.2 were used to determine the pressures gradients experienced by the materials in the nip (no-slip) and slip regions during roller compactions (Johanson, 1965, Reynolds et al., 2010). The equations were solved to determine the nip angle $(\alpha)$ where the boundary condition between the material and the rolls changes from a slip condition to a nip condition (see Figure 3.4) (Reynolds et al., 2010, Souihi et al., 2015). By using Equation 3.3 and Equation 3.4, the $\delta \mathrm{E}$ and $\varphi_{\mathrm{w}}$ values for each material were used to determine parameters $\mathrm{A}$ and $v$.

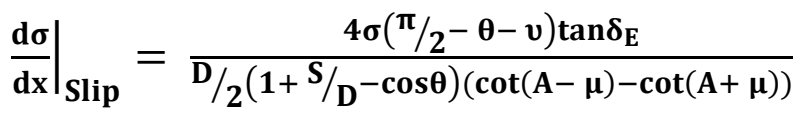




$$
\left.\frac{\mathrm{d} \sigma}{\mathrm{dx}}\right|_{\text {Nip }}=\frac{K \sigma_{\theta}(2 \cos \theta-1-S / D) \tan \theta}{\mathrm{D} / 2\left(1+S / D^{-\cos \theta) \cos \theta}\right.}
$$

Equation 3.2

where

$$
\begin{gathered}
A=\frac{\theta+v+\frac{\pi}{2}}{2} \\
2 v=\pi-\arcsin \left(\frac{\sin \phi_{w}}{\sin \delta_{E}}\right)-\phi_{w}
\end{gathered}
$$

Equation 3.4

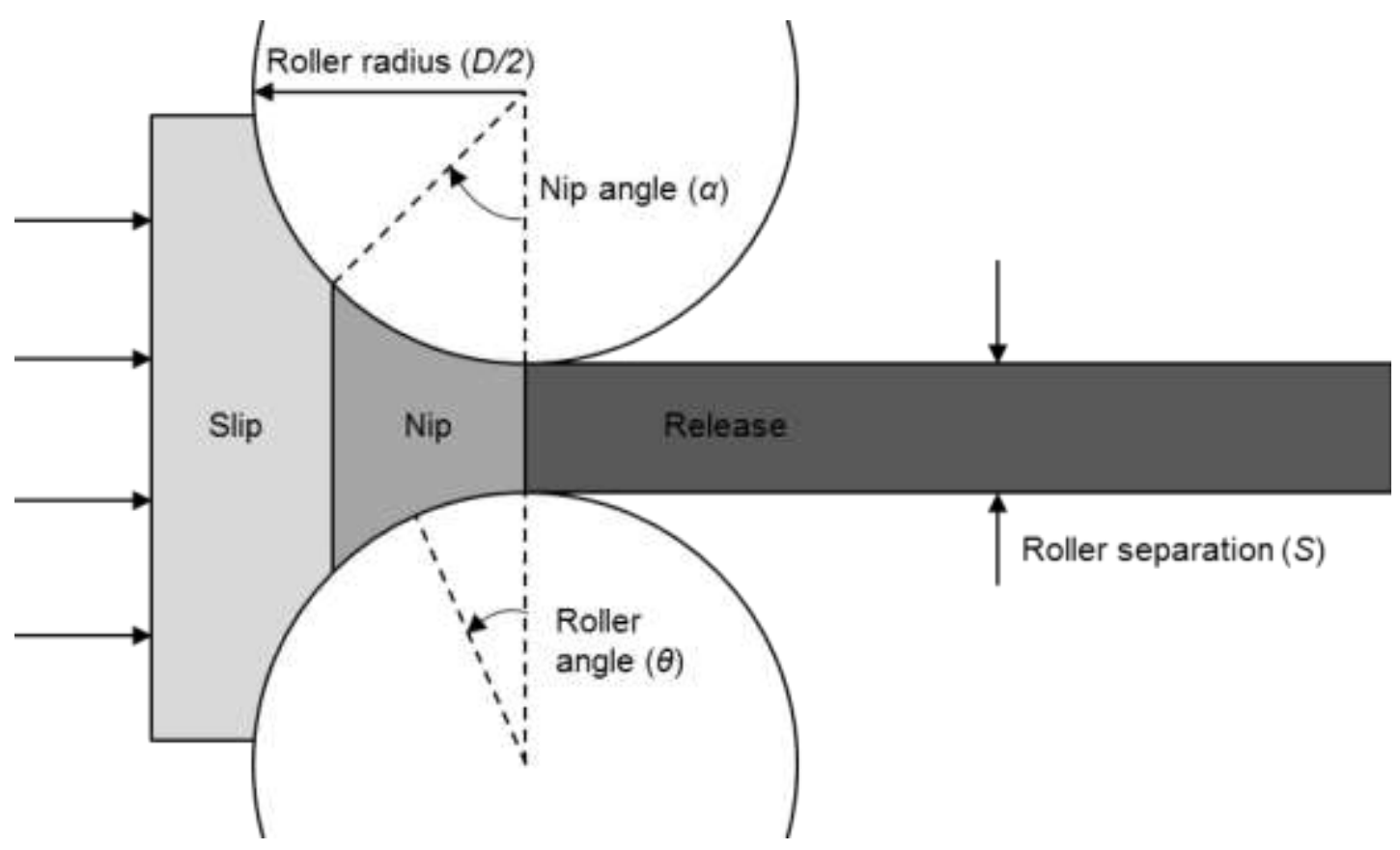

Figure 3.4 Schematic diagram showing the roller compaction process.

To relate the hydraulic pressure $\left(\mathrm{R}_{\mathrm{f}}\right)$ applied to the material to the peak pressure $\left(\sigma_{R C}\right)$ Equation 3.5 and Equation 3.6 were used which represent the relationship between the processing parameters used. By determining the compressibility $(\mathrm{K})$ and the nip angle $(\alpha)$ as mentioned previously, Equation 3.5 and 
Equation 3.6 can be solved to relate the applied hydraulic pressure $\left(R_{f}\right)$ to the peak pressure (бRC) as stress.

$$
\begin{gathered}
\mathbf{R}_{\mathrm{f}}=\frac{\sigma_{\mathrm{RC}} \mathrm{WDF}}{2} \\
F=\int_{\theta=0}^{\theta=\alpha\left(\delta_{E}, \emptyset_{E}, K\right.}\left[\frac{S / D}{\left(1+S / D^{-\cos \theta) \cos \theta}\right]^{K} \cos \theta d \theta}\right.
\end{gathered}
$$

Equation 3.6

where $\mathrm{W}$ is the roller width, $\mathrm{D}$ is the roller diameter, $\mathrm{S}$ is the roller separation and $\mathrm{K}$ is the compressibility. In this study the values used were as follows; $\mathrm{W}=0.04$ $\mathrm{m}, \mathrm{D}=0.12 \mathrm{~m}$ and $\mathrm{S}=0.003 \mathrm{~m}$.

The compressibility factor $(\mathrm{K})$ in terms of the tablet density was determined for the Man100:MCC0 and Man0:MCC100 formulations by using Equation 3.7.

$$
\log \sigma_{\mathrm{tab}}=\frac{1}{\mathrm{~K}} \log \rho_{\mathrm{tab}}+\log \gamma_{0}
$$

Equation 3.7

where $\sigma_{\mathrm{TS}}$ is the tableting stress, $\mathrm{K}$ is the compressibility, Qtab is the tablet density and $\gamma_{0}$ is the preconsolidation relative density. The $\delta_{\mathrm{E}}, \varphi_{\mathrm{w}}$ and $\mathrm{K}$ values used in the calculations are stated in Table 3.3.

Table 3.3 The effective angle of internal friction $\left(\delta_{E}\right)$, the angle of wall friction $\left(\varphi_{\mathrm{w}}\right)$ and compressibility (K) values.

\begin{tabular}{|cccc|} 
& $\delta_{\mathrm{E}}\left({ }^{\circ}\right)$ & $\boldsymbol{\phi}_{\mathrm{w}}\left({ }^{\circ}\right)$ & $\mathrm{K}$ \\
\hline Mannitol & 36.1 & 8.9 & 7.2 \\
\hline MCC & 44.5 & 6.8 & 4.8 \\
\hline
\end{tabular}


Table 3.4 shows the values obtained for the RC stress ( $\left.\sigma_{R C}\right)$; these values can then be related with the tableting stress $(\sigma \mathrm{TS})$ to determine the different degrees of stress applied during the production of a tablet, i.e. during roller compaction and tableting.

Table 3.4 The values for the stress applied when roller compaction pressures of 20 and $\mathbf{1 0 0}$ bar are used during granulation for Man100:MCC0 and Man0:MCC100 formulations.

\begin{tabular}{|c|cc|}
\hline Abbreviations & $\begin{array}{c}\text { RC hydraulic pressure } \\
\text { used (bar) }\end{array}$ & $\sigma_{\mathrm{RC}}$ RC stress (MPa) \\
\hline Man100:MCC0 & 20 & 40 \\
\hline Man100:MCC0 & 100 & 199 \\
\hline Man0:MCC100 & 20 & 34 \\
\hline Man0:MCC100 & 100 & 171 \\
\hline
\end{tabular}

\subsubsection{Particle size distribution}

The size distributions of the granules were determined using a Sympatec QICPIC (Clausthal-Zellerfeld, Germany) particle size analysis apparatus, fitted with the VIBRI vibratory feeding unit and GRADIS dispersion unit. The QICPIC utilises a high speed camera with a short exposure time to obtain images of the particles. The images are then processes using WINDOX software. 5 measurements $(5.0 \mathrm{~g}$ samples) were carried out for each granule batch. The QICPIC system is capable of measuring particles in the size range of $1-34000 \mu \mathrm{m}$. The volume-weighted particle size distributions and the $\mathrm{D}_{10}, \mathrm{D}_{50}$ and $\mathrm{D}_{90}$ values were obtained. 


\subsubsection{Tableting}

The second processing parameter that was varied and examined in this research was the tableting stress. This is the second stress which is applied to a material during the manufacture of tablets.

Tablets were produced using an Instron 3367 (Buckinghamshire, UK) with a purpose-built flat face upper punch and stationary $12 \mathrm{~mm}$ die. Approximately 500 mg of granules were weighed and filled into the die manually (see Figure 3.5). The material was compressed using a loading rate of $1 \mathrm{~mm} / \mathrm{s}$, with no dwell time following compression. Magnesium stearate was used as an external lubricant during tableting to prevent sticking. Tablets were produced using 5 and $20 \mathrm{kN}$ to ensure that the tablets exhibited varying properties. This ensured that tablets with a range of tensile strengths and porosities were formed for analysis.

Upper punch
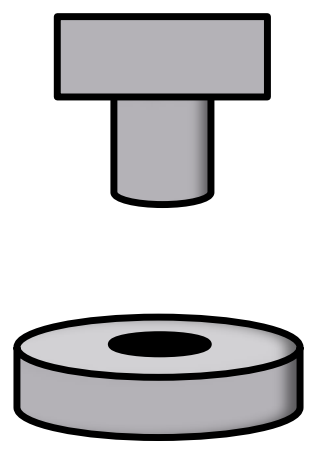

Tablet die
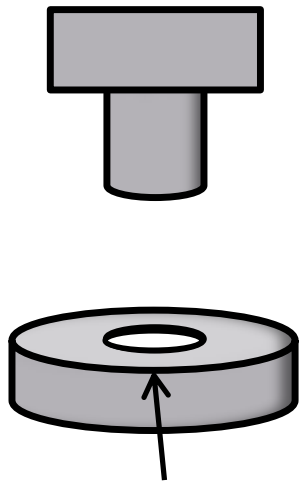

Granules
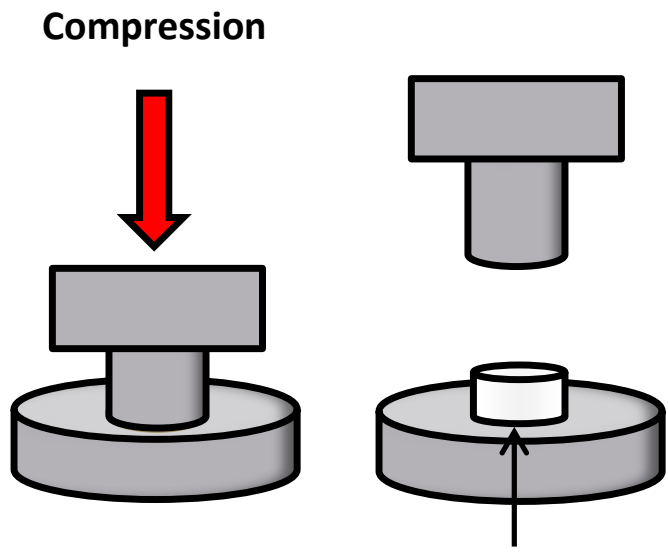

Tablet

Figure 3.5 Diagram showing compression of granules into a tablet.

The stress applied during tableting was also calculated by dividing the force by the area of the tablet. 
Table 3.5 The values for the force used during tableting, and the equivalent stress.

\begin{tabular}{|cc|}
\hline Tableting force $(\mathrm{kN})$ & Tableting stress $(\mathrm{MPa})$ \\
\hline $\mathbf{5}$ & 44.2 \\
\hline $\mathbf{1 0}$ & 88.4 \\
\hline $\mathbf{2 0}$ & 176.8 \\
\hline
\end{tabular}

\subsubsection{Tablet tensile strength}

Tablet tensile strength $\left(\sigma_{\mathrm{T}}\right)$ was calculated using Equation 3.8 (Fell and Newton, 1968), by measuring the force required to break the tablet ( $\left.F_{\max }\right)$. The $F_{\operatorname{maz}}$ was determined using the Zwick-Roell (Ulm, Germany) instrument. The tablet dimensions were measured using a precision slider calliper.

$$
\sigma_{\mathrm{T}}=\frac{2 \mathrm{~F}_{\mathrm{max}}}{\pi \mathrm{DT}}
$$

\section{Equation 3.8}

where $\sigma_{\mathrm{T}}$ is the tablet tensile strength, $\mathrm{D}$ is the tablet diameter and $\mathrm{T}$ is the tablet thickness.

\subsubsection{X-ray tomography}

X-ray tomography was utilised to determine ribbon and tablet porosity. A SCANCO Medical MicroCT35 X-ray (SCANCO, Switzerland) was used to obtain Xray images. For ribbons a section of ribbon of $10 \mathrm{~mm}$ length was obtained and X-ray measurement was carried out at the centre of the ribbon. For tablets the middle of a $12 \mathrm{~mm}$ tablet was scanned. To determine the porosity the images obtained were analysed using ImageJ software. By using ImageJ the grey value histograms for blank X-rays (which contained only air) and the sample X-rays were extracted, 
where 0 is the darkest possible shade (i.e. black). For the blank air measurement the histogram for the grey level was calculated to reflect the percentage of the total counts. It was assumed that at $99.9 \%$ of the total, the grey level is air and anything above is particulate. By using the grey level value obtained as being air, the porosity was calculated.

\subsection{Results and Discussion}

\subsubsection{Ribbon porosity}

X-ray tomography was utilised to measure ribbon porosity. Porosity is an indication of the percentage of air present in a solid system. Ribbon porosity was examined to determine the effect of compaction stress at the granulation stages on ribbon porosity.

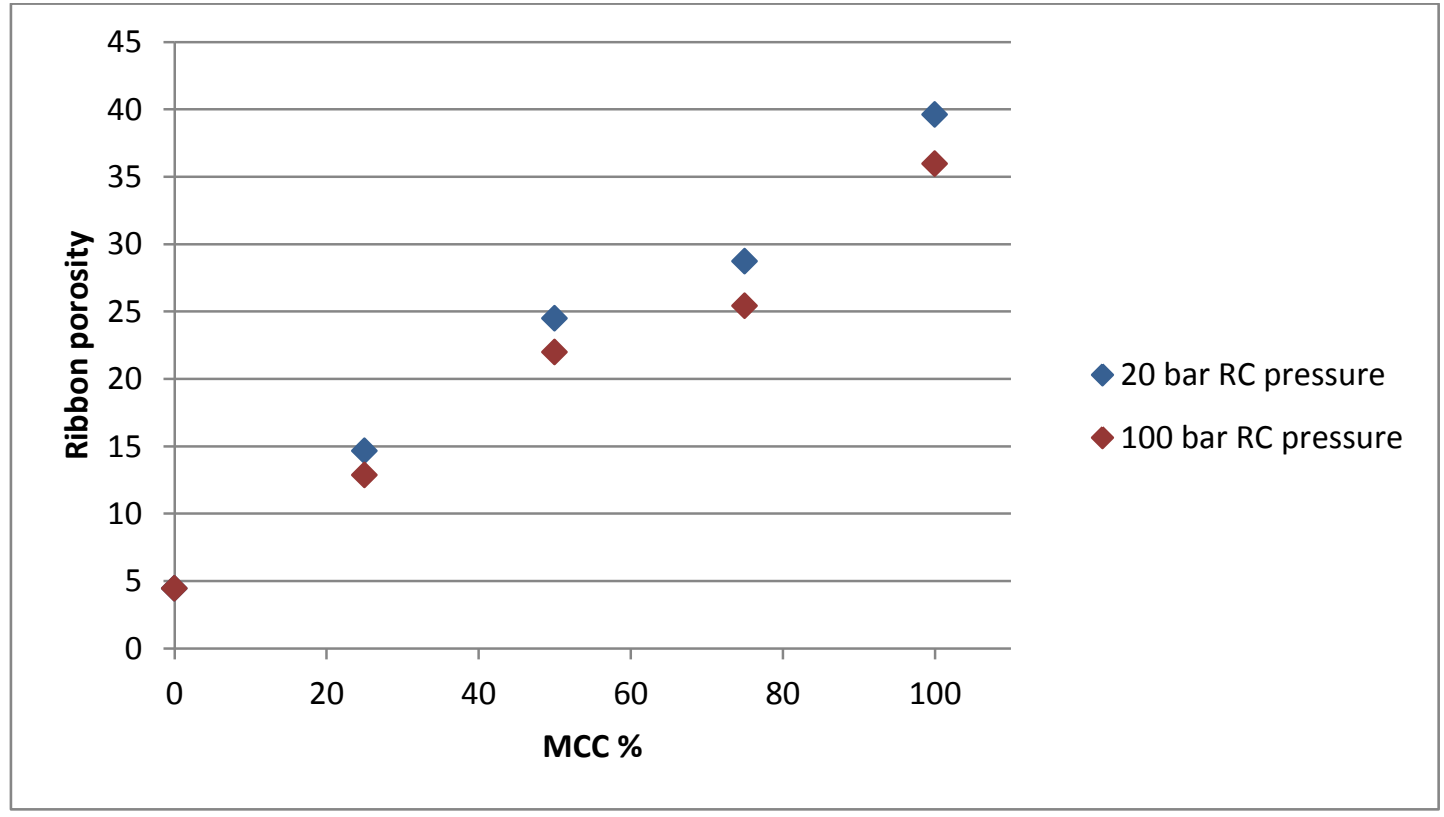

Figure 3.6 Ribbon porosities calculated for Man100:MCC0, Man75:MCC25, Man50:MCC50, Man25:MCC75 and Man0:MCC100 ribbons which were produced using 20 or 100 bar roller compaction pressure. 
The porosities for ribbons produced using Man100:MCC0, Man75:MCC25, Man50:MCC50, Man25:MCC75 and Man0:MCC100 formulations were determined. Figure 3.6 shows the ribbon porosities when RC pressures of 20 or 100 bar are used during granulation. Man100:MCC0 ribbons are the least porous in terms of the material used. When a higher RC pressure is used the ribbons were found to be less porous. This is due to the higher stress being applied to the material causing higher densification of the material.

\subsubsection{Granule size distribution}

The size distributions for mannitol granules and MCC granules, produced using varying roller compaction $(\mathrm{RC})$ pressures, are shown in Figure 3.7 and Figure 3.8, respectively. Firstly, it can be seen that in every case a bimodal particle size distribution was attained. For both MCC and mannitol granules a low RC pressure of 20 bar led to a higher percentage of smaller granules than a when a high RC pressure (100 bar) is used. Additionally, a higher RC pressure produced a higher percentage of larger granules than when a lower RC pressure is used. In the case of mannitol granules, the increase of RC pressure led to the size distribution shifting towards a unimodal distribution as the percentage of smaller particles decrease significantly with the addition of a higher RC pressure and consequently better compaction of the powder. 


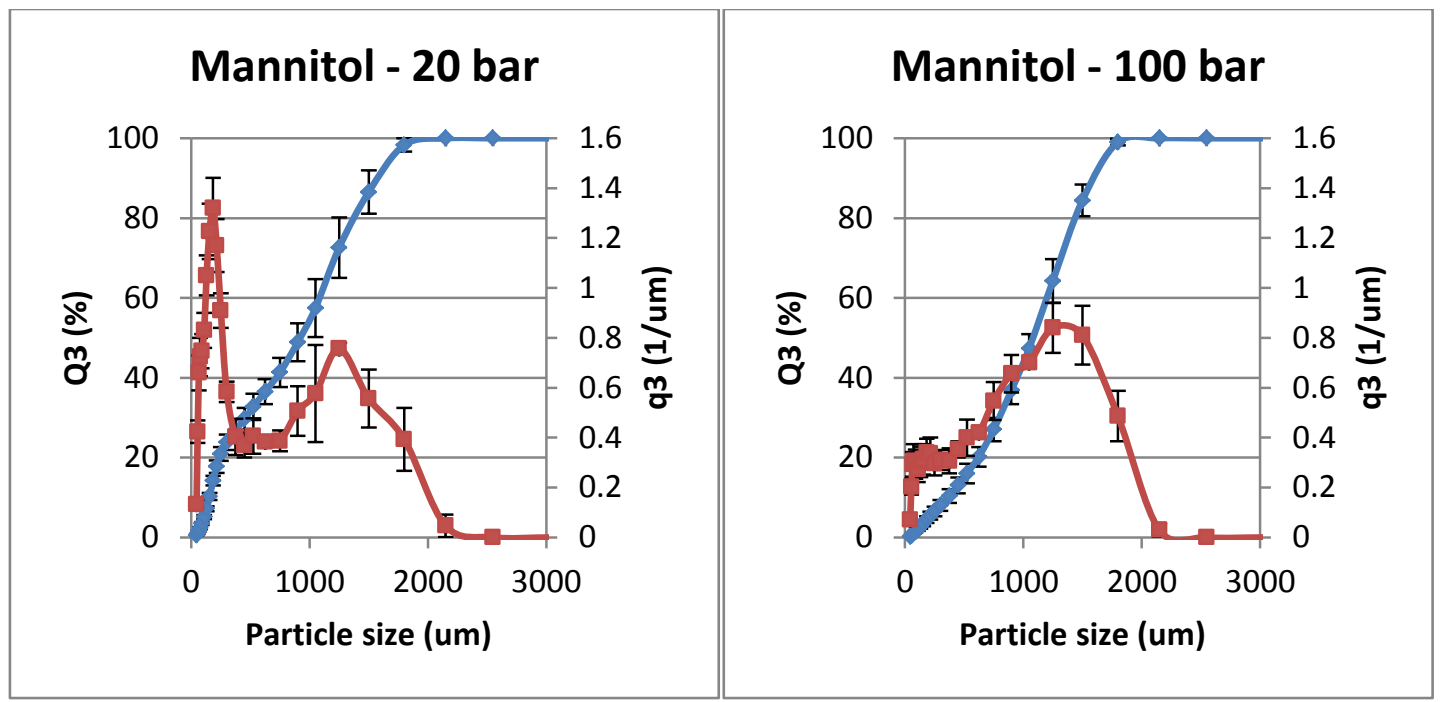

Figure 3.7 Granule size distribution data for mannitol granules produced using roller compaction at various pressures.

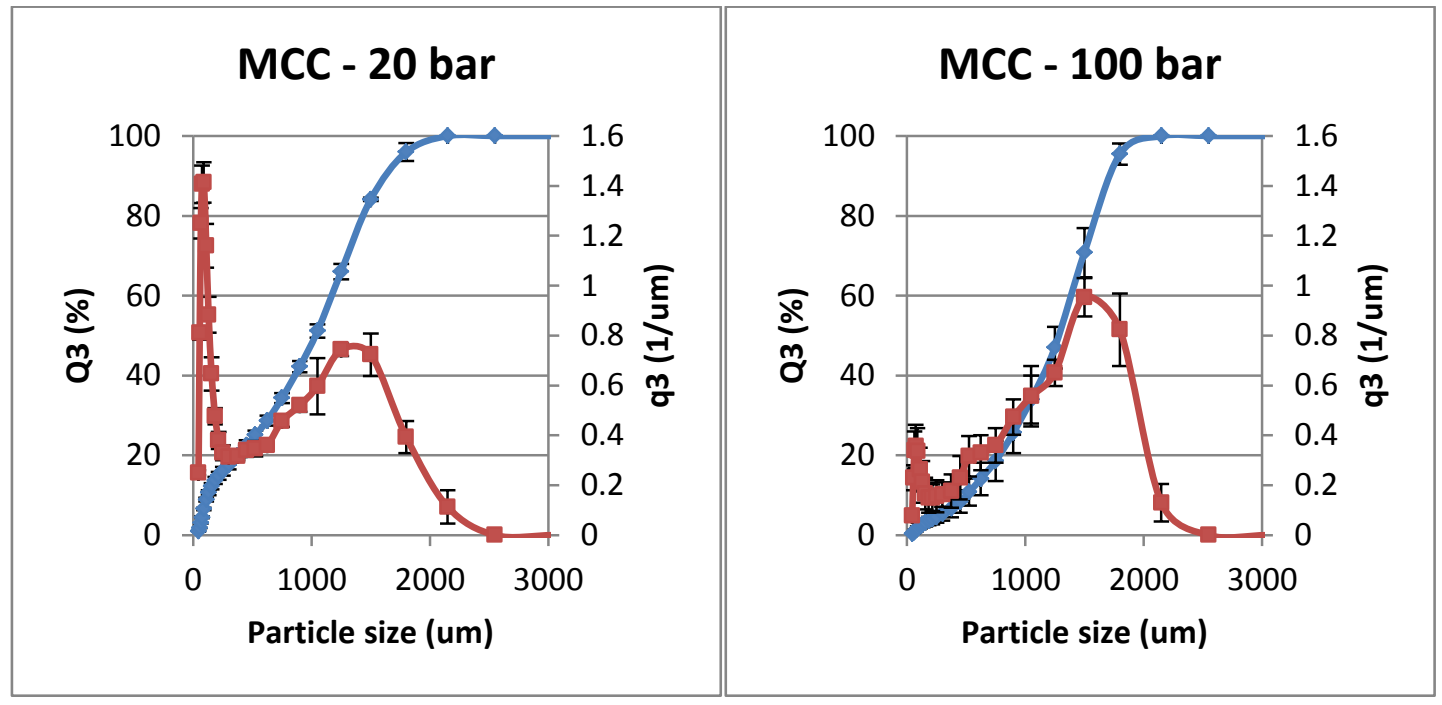

Figure 3.8 Granule size distribution data for MCC granules produced using roller compaction at various pressures.

The $\mathrm{D}_{10}, \mathrm{D}_{50}$ and $\mathrm{D}_{90}$ values for mannitol granules and MCC granules are shown in Figure 3.9 and Figure 3.10, respectively. MCC granules produced using roller compaction are generally larger than mannitol granules produced using the same conditions. With increasing $\mathrm{RC}$ pressure the $\mathrm{D}_{10}$ particle size increases 
significantly for both MCC and mannitol granules. The $\mathrm{D}_{50}$ and $\mathrm{D}_{90}$ values also increase with increasing RC pressure, however not as drastically as the $\mathrm{D}_{10}$ values.

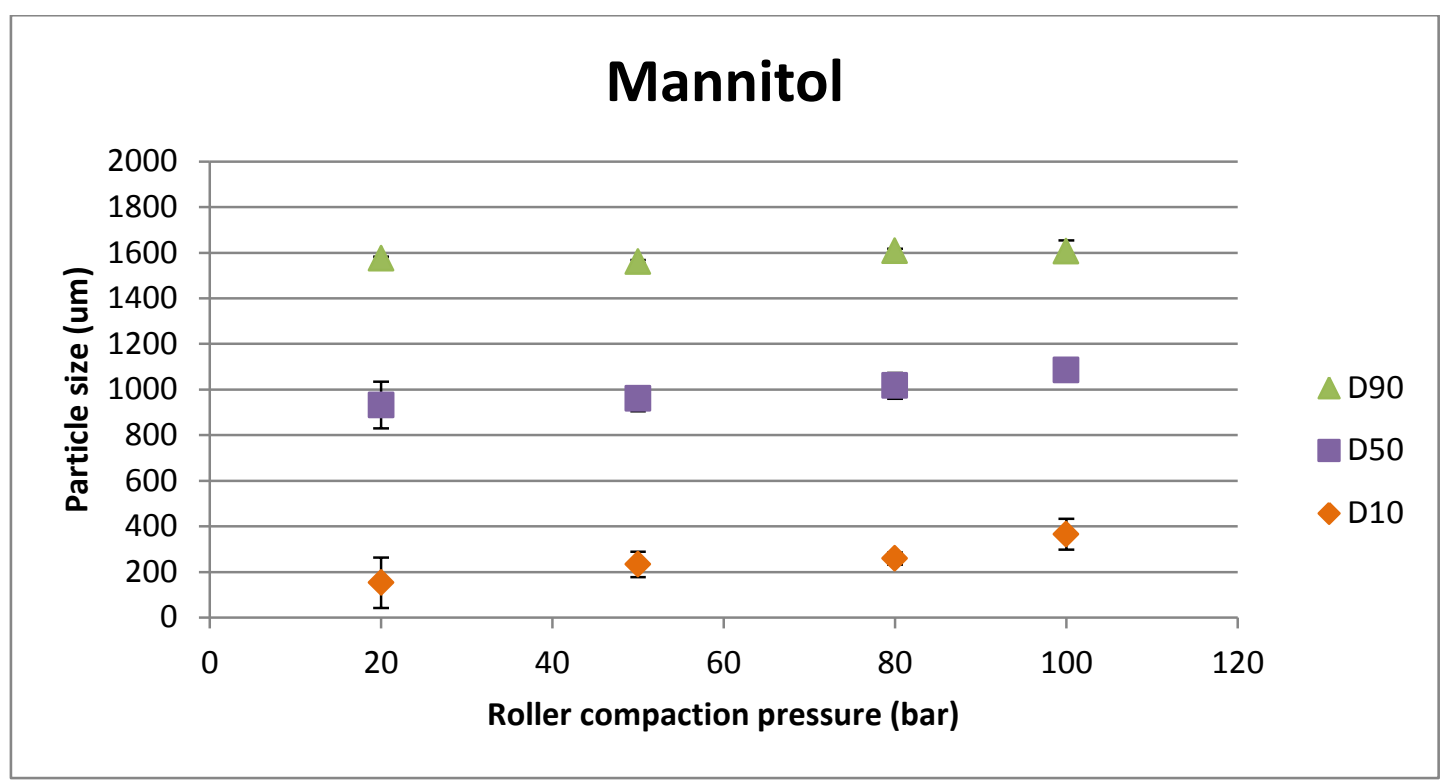

Figure $3.9 D_{10}, D_{50}$ and $D_{90}$ values for mannitol granules produced using roller compaction at various pressures.

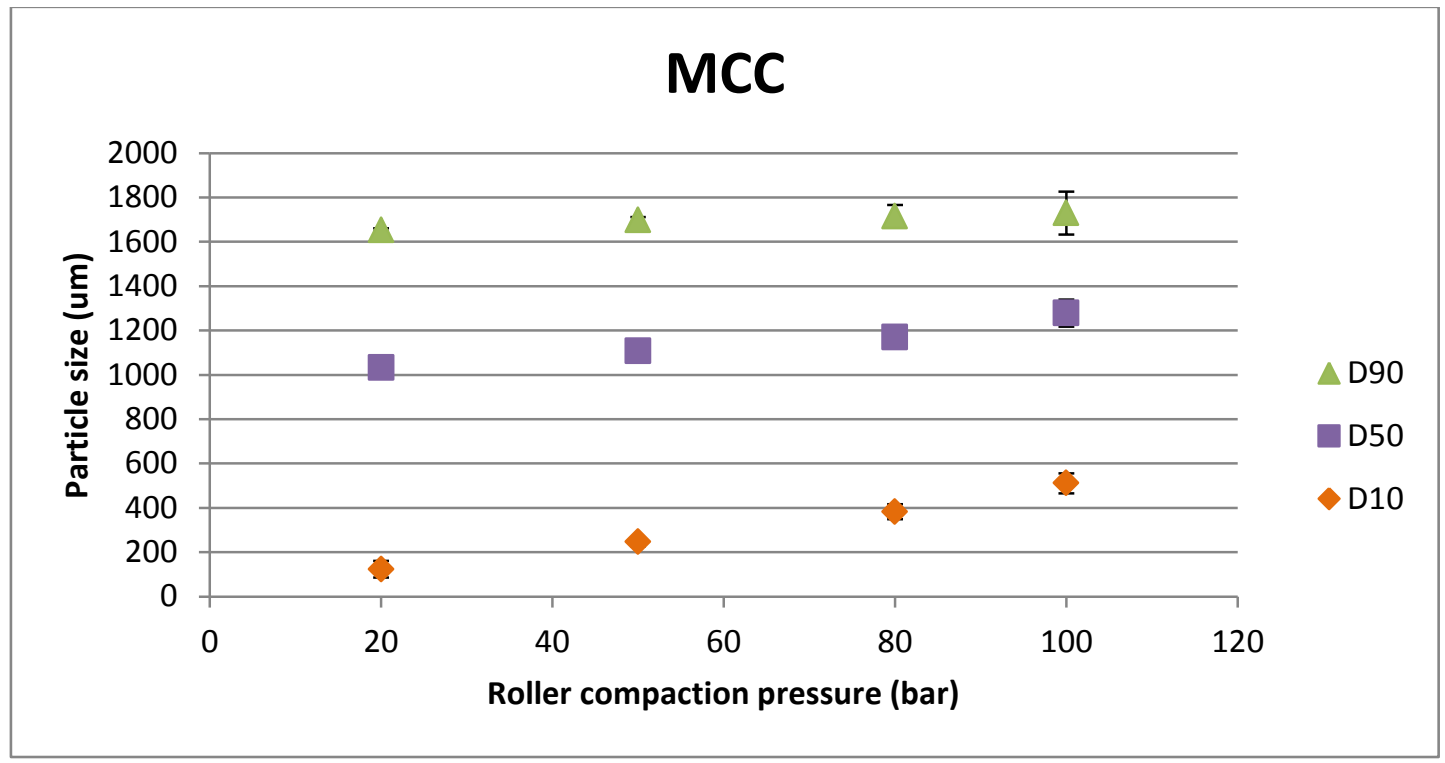

Figure $3.10 \mathrm{D}_{10}, \mathrm{D}_{50}$ and $\mathrm{D}_{90}$ values for $\mathrm{MCC}$ granules produced using roller compaction at various pressures. 
A decrease in the percentage of smaller particles with increasing granulation pressure is fundamentally due to the application of stress to the primary powder leading to the formation of bonds. With the application of higher stress stronger bonds are formed and the material is more densified during the production of ribbons (Khorasani et al., 2016). When the ribbons are then crushed to form granules, ribbons produced with higher RC pressure form larger granules and less fines due to the strong bond formation. Ribbons produced with less RC pressure are weaker and produce more fines during the compaction process and then additionally more fines during the crushing process.

\subsubsection{Tablet compactibility}

Compactibility graphs are shown (Figure 3.11) for the tablets produced from granules roller compacted using 20 and 100 bar, and tableted using force of 5 and 20 $\mathrm{kN}$. At a low tableting force $(5 \mathrm{kN})$ the effect of $\mathrm{RC}$ pressure is more pronounced in terms of the tablet porosity, whereas the tensile strength of the tablets is not changing as significantly. Higher RC pressures led to tablets with a lower porosity and lower tensile strength.

To explain these trends the properties of the granular material must be considered. A higher RC pressure used during granulation means the material undergoes higher stress and therefore forms stronger intra-granular bonding (compared to material granulated using a low RC pressure). Therefore, the resultant granular material is dense (see Figure 3.6). The application of low stress during tableting means that the tablet is unable to form strong particle-particle bonding as the granular material is not able to be crushed; the stress is insufficient (see Table 
3.4). The lower porosity of tablets containing high $\mathrm{RC}$ granules may be an indication of the granular porosity. This will be examined further in the following Section.

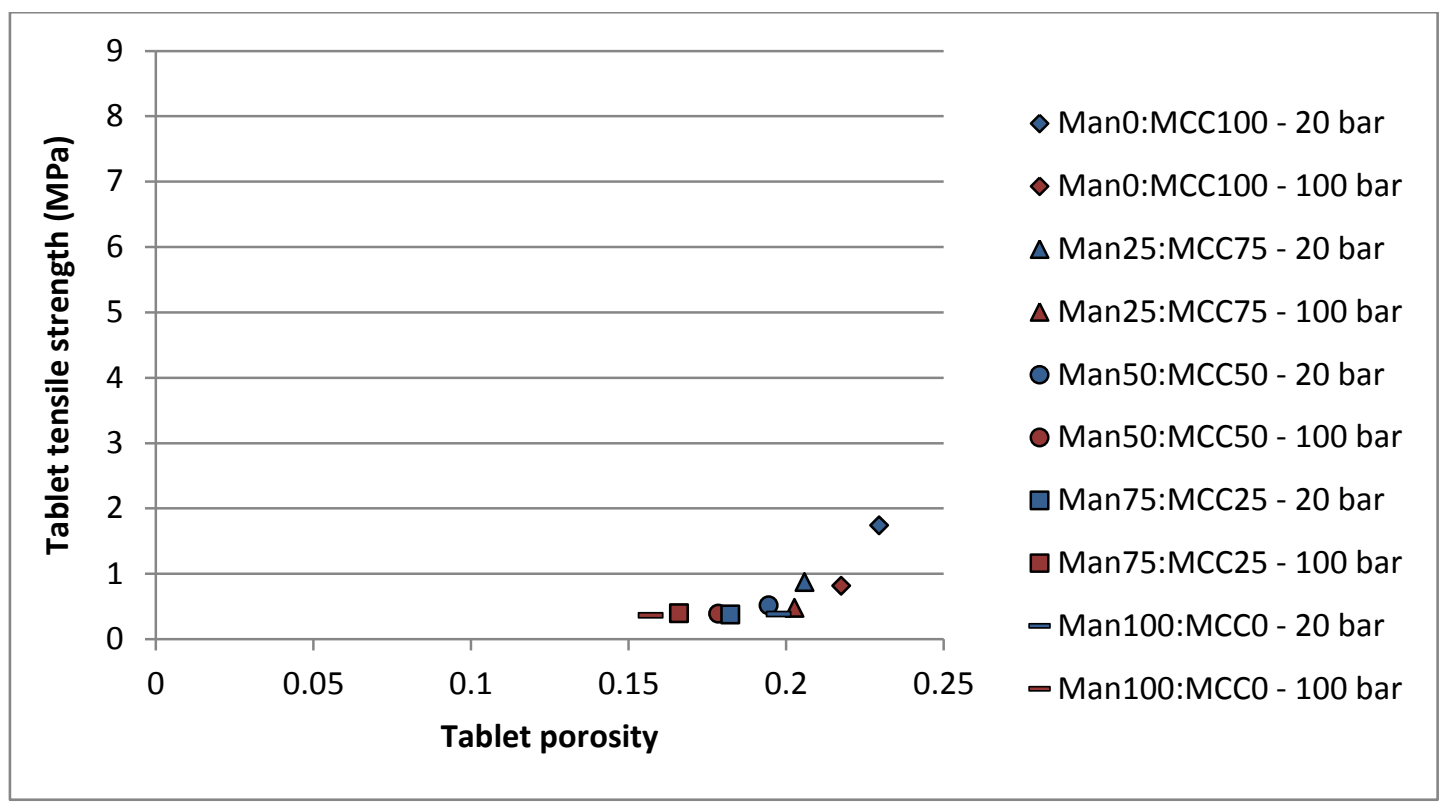

Figure 3.11 Compactibility graph for tablets produced using varying ratios of mannitol and MCC, using either 20 or 100 bar RC pressures and using $5 \mathrm{kN}$ tableting force.

At a $20 \mathrm{kN}$ tableting force the porosity and tensile strength also decreased when increasing the RC pressure (see Figure 3.12). Granules produced using higher RC stress can withstand even higher tableting stresses due to the granular material itself being stronger. This may be why the higher RC granules produce weaker tablets even at higher tableting forces. Previously in literature this phenomenon has been attributed to the "loss in compactibility" of a material, i.e. due to the work hardening of a material during granulation.

The effect of tableting force is evident (see Figure 3.11 and Figure 3.12); a higher tableting stress leads to a lower tablet porosity and a higher tablet tensile strength, as seen in the literature (Razavi et al., 2016, Schmidtke et al., 2017, Hadžović et al., 2011). This is fundamentally due to the further densification of the 
granular material during the application of the tableting stress, which leads to a lower tablet porosity and stronger particle-particle bonding.

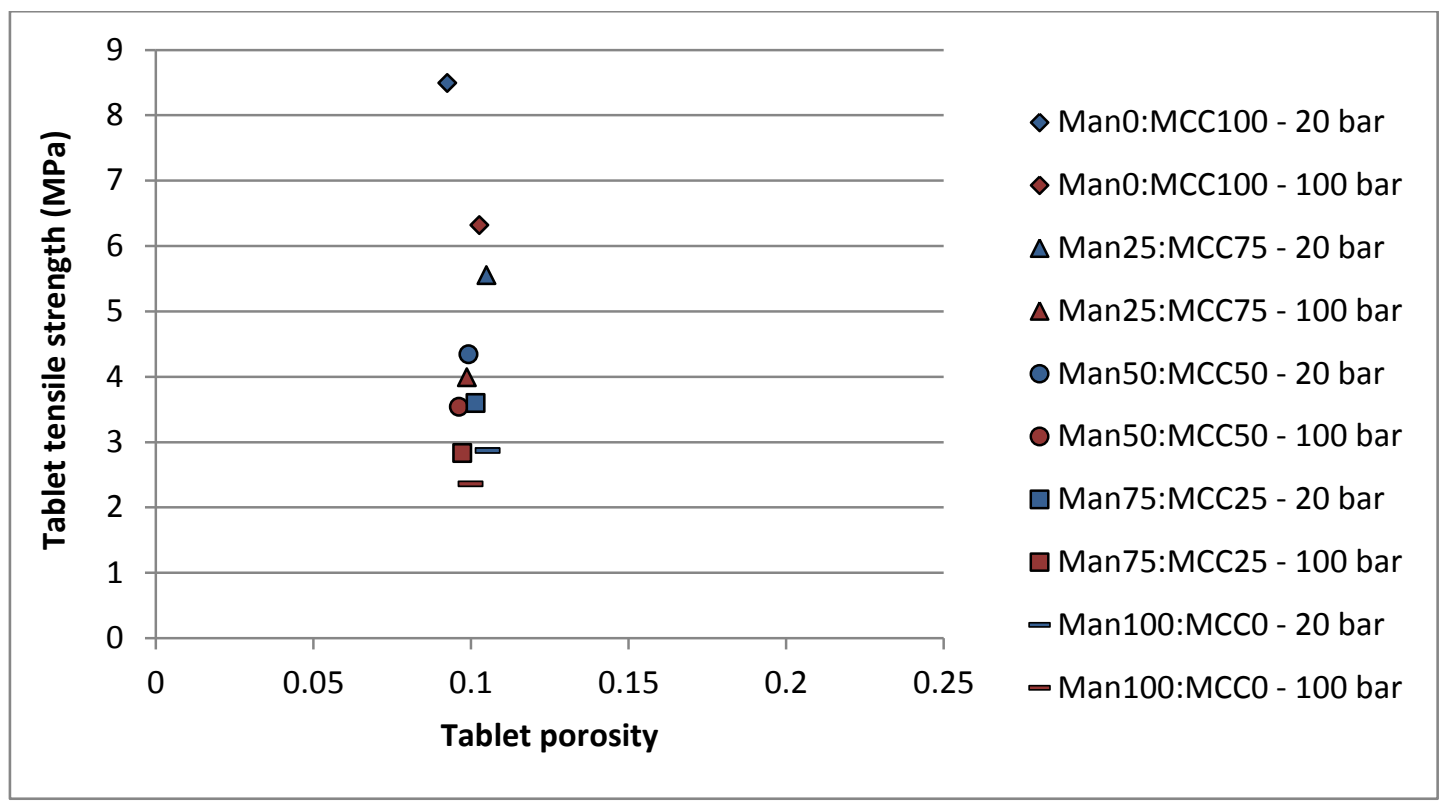

Figure 3.12 Compactibility graph for tablets produced using varying ratios of mannitol and MCC, using either 20 or 100 bar RC pressures and using $20 \mathrm{kN}$ tableting force.

In the case of the MCC percentage, the tablet tensile strength increases with increasing MCC in all cases (see Figure 3.11 and Figure 3.12). The increase in tablet tensile strength is more prominent with increasing tableting force, for a given RC pressure with increasing MCC in the formulation. This may be due to MCC ribbons being significantly more porous than mannitol ribbons (see Figure 3.6), therefore the stress applied during tableting is able to crush more of the granular material which contains a higher percentage of MCC and form stronger inter-particle bonding. At low tableting load, increasing MCC percentage causes the tablet porosity to increase. This will be examined further in the next Section. 


\subsubsection{Tablet internal structure}

The compactibility graphs established that the amount of stress applied to the material during roller compaction affects the granular properties. The granular properties in turn affect the tablet properties, depending on whether the tableting stress applied is higher or lower than the granulation stress. The internal structure within the tablet is a key feature which will validate the findings previously outlined. The internal structure gives indication of the bonding occurring within a tablet, depending on the material and stressed applied at each stage. By using X-ray tomography it was possible to visualise the granular material within the tablets and determine to what extent the granular structure is maintained during the tableting process. The black colour within the X-ray images signifies the presence of air and a stronger white colour signifies the presence of material.

From the images (Figure 3.13) it can be observed that at low tableting force (5 $\mathrm{kN}$ ) the Man100:MCC0 granular material is still visible within the tablets, to varying degrees depending on the RC pressure used to produce the granule. A higher RC pressure during granulation leads to the formation of less porous granule (see Figure 3.6); from the X-ray it can be observed that the high RC stress granular material integrity is still maintained after the tableting stress is applied. This is due to the tableting stress applied to the material being insufficient to crush the granular structure (see Table 3.4 and Table 3.5). On the other hand, a low tableting force applied to granules produced using a low RC pressure led to a high percentage of the relatively weak granular material within the tablet being crushed. The equivalent tablet stress was higher than the stress applied during RC, therefore more of the granular material is likely to be crushed. 
In Figure 3.11 the effect of RC pressure was evident in only the tablet porosity, with higher RC pressure producing tablets with lower porosity. The X-ray evidence suggests that the granular structure within a tablet dominates the overall measured tablet porosity. When a high RC stress granule is present and the tableting stress is low then the granular structure is prevalent within the tablet and the bonding within the tablet is weak. Therefore, the tablet contains low porosity granules and pores between the granules (see Figure 3.13). But due to the nature of the X-ray measurement, which is carried out on the whole tablet, the average of the granular porosity and the inter-granular porosity within the tablet is considered as the total tablet porosity. So tablets visibly containing low porosity granules are also calculated as having a low tablet porosity.

The compactibility graphs (see Figure 3.11) also showed that when higher RC stress granules are tableted using a low stress, the consequent tablets are more porous and also weaker than when low RC stress granules are used. The X-ray suggests that this is due to the low RC stress granular material being more readily crushed, therefore providing a higher surface area for bonding. Whereas the low tableting stress is not sufficient to crush the higher RC stress granules or form strong inter-granular bonding.

With increasing MCC percentage in the tablet, there appears to be an increase in the pores which exist in the tablet (see Figure 3.13). This may explain the higher tablet porosity witnessed in the previous Section (see Figure 3.11). The porosity was measured using X-ray; therefore an average of the tablet granules and pores which exist in the tablet were observed. 


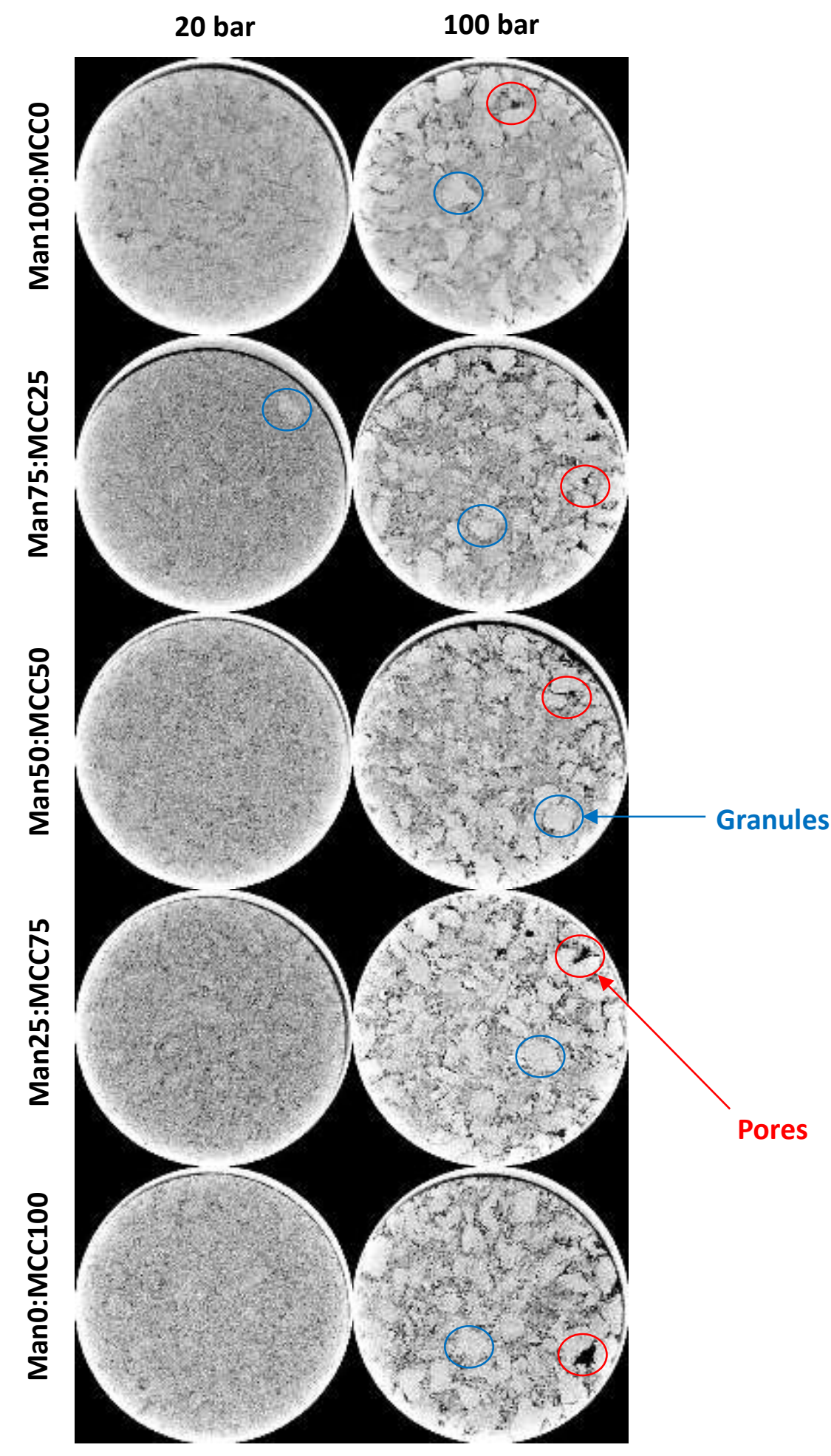

Figure 3.13 X-ray tomography images of tablets with varying ratios of mannitol and MCC, which were compacted using $5 \mathrm{kN}$ tableting force and 20 and $100 \mathrm{bar}$ RC pressure granules. The images presented were obtained from the centre of the tablet and the colour altered for the purpose of presentation to highlight any variation in the internal densities. Examples of the granular material and pores present within the tablet are highlighted. 
When the tableting force is increased to $20 \mathrm{kN}$ (see Figure 3.14), the X-ray images for tablets produced using granular material of a low RC pressure (20 bar) show that no granular material is present after tableting. This suggests that the tableting stress is not only high enough to crush the relatively weak granular material, but also high enough to promote strong bonding within the tablet (see Table 3.4 and Table 3.5). From the compactibility graphs (see Figure 3.12) it was observed that the tablet tensile strength was dependent on the RC pressure used to produce the granules, whereas the tablet porosity was largely unaffected. The X-ray imaging suggests that at higher tableting force the granular material is predominantly crushed, meaning a larger surface area is available for particleparticle bonding. The compactibility graphs confirm that the low RC pressure in addition to a high tableting force leads to the production of the densest and strongest tablets.

In the case where a high RC pressure of 100 bar is used during granulation, a high tableting force of $20 \mathrm{kN}$ is still insufficient to crush all granular material (see Figure 3.14); in some cases granular material is still visible. At higher tableting force, granules produced using a higher RC stress form tablets which are weaker than tablets containing low RC stress granules (Figure 3.12), possibly due to the lower surface area available for strong inter-granular bonding to occur. 


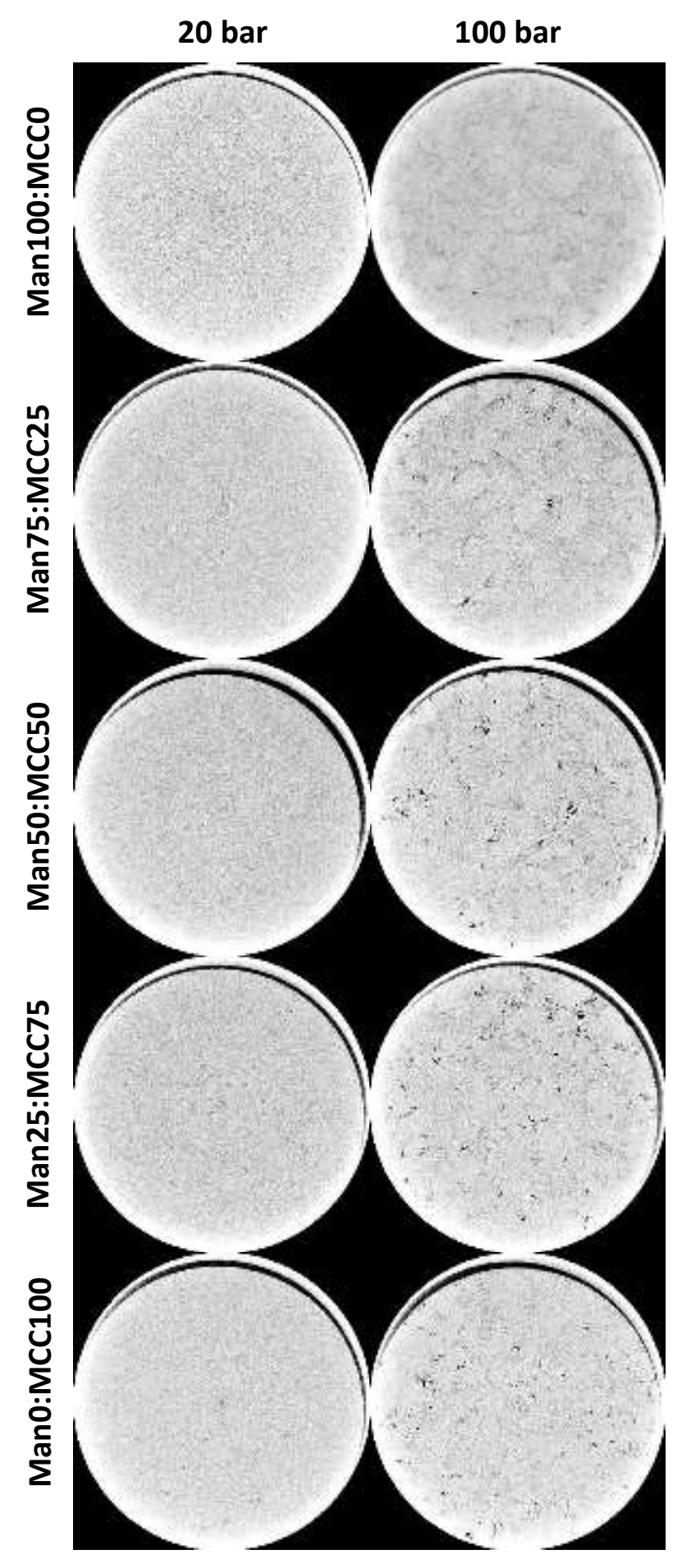

Figure 3.14 X-ray tomography images of tablets with varying ratios of mannitol and MCC, which were compacted using $20 \mathrm{kN}$ tableting force and 20 and 100 bar RC pressure granules. The images presented were obtained from the centre of the tablet and the colour altered for the purpose of presentation to highlight any variation in the internal densities. 
From the X-ray imaging it is clear that the granular porosity greatly influences the tablet porosity when low tableting stresses are used. The presence of dense granular material after tableting leads to denser tablets, and inversely the presence of porous granular material leads to more porous tablets (see Figure 3.11 and Figure 3.12). It should be noted that tablets containing 100 bar granules were measured to be less porous due to the granular material being denser. However, the pores between the 100 bar granules are significantly larger than for 20 bar granules. On contact with water these pores will allow for rapid uptake of water deep within the tablet, even though the calculated porosity is lower.

The findings in this Chapter are a divergence from what is reported in literature which state that the reduction in tensile strength, or "loss in compressibility", when a higher RC pressure is used is due to "work hardening". The literature suggests that the work hardening increases with increasing particle size from the same ribbon, i.e. the same density (Herting and Kleinebudde, 2008, Mosig and Kleinebudde, 2015, Perez-Gandarillas et al., 2016). The X-ray images suggest that the tablet tensile strength is based on whether the tableting stress is sufficient enough to crush the granular material, and then form strong particleparticle bonding (see Figure 3.15). This is dependent on the porosity of the granular material. 


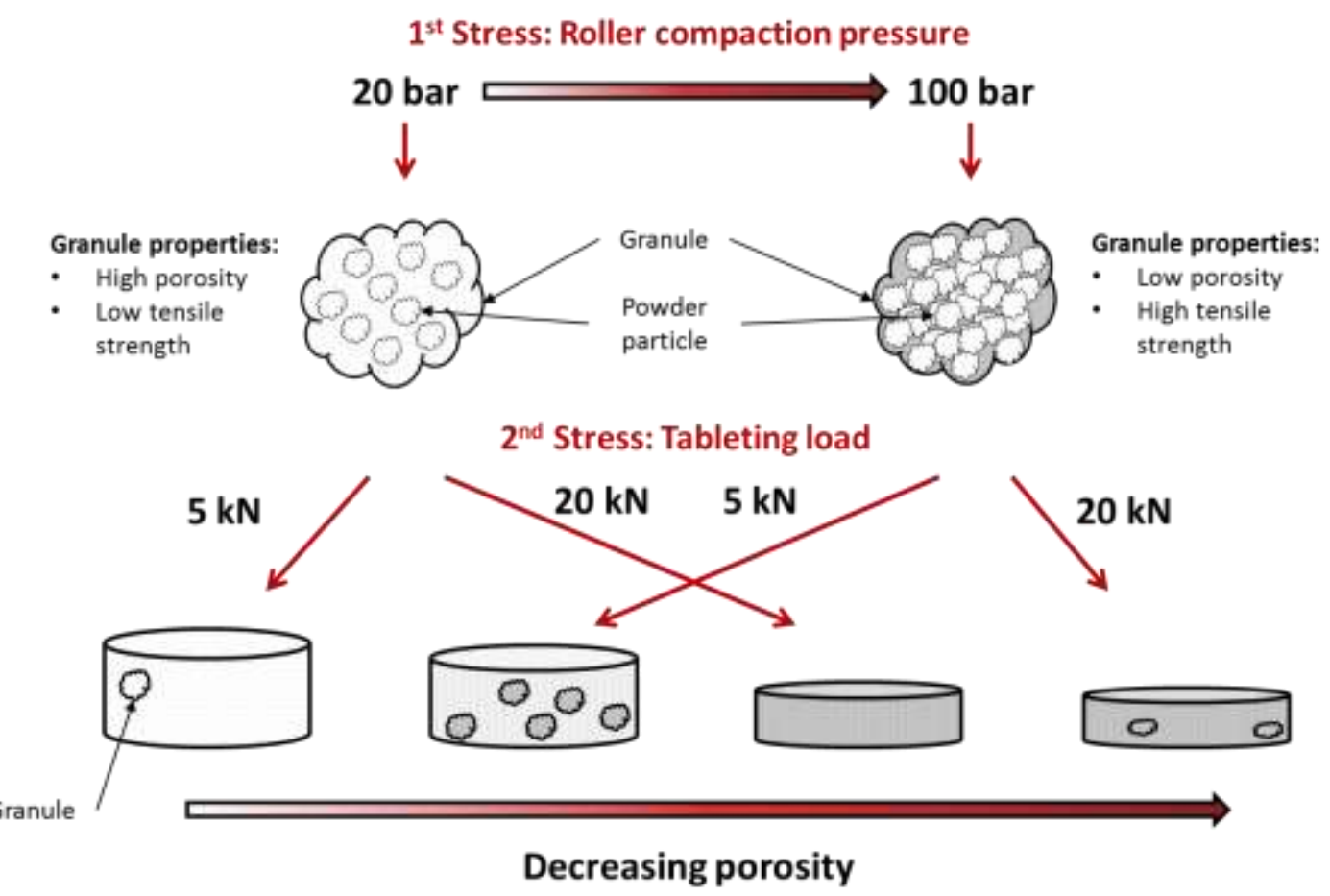

Figure 3.15 Schematic of granule packing after tableting depending on the roller compactor pressure.

\subsection{Conclusions}

From this research it was found that tablet compactibility is dependent on the RC stress to varying degrees, depending on the tableting stress used to compact the granules (see Figure 3.15). At low tableting stress the tablet properties are dominated by the granular properties in terms of strength and porosity. This is because the tableting stress is insufficient to crush the granular material, which then still exists within the tablet and influences the tablet porosity.

When a higher tableting stress is used the 20 bar granular structure within a tablet was in most cases eliminated. Generally, a significant increase in the tablet tensile strength was observed, and the tablet porosity was found to be largely unaffected by the RC pressure used. A decrease in tablet tensile strength was 
observed when higher RC pressure granules were used. In literature this is termed as "loss in compressibility" of a material due to compaction of the material during the granulation stage. In this research it was proposed that the reduction in tensile strength is related to whether the tableting stress is sufficient enough to firstly break the granular material, and secondly form strong particle-particle bonding.

Generally, the addition of MCC caused the tablets to increase in tensile strength, to varying degrees depending on the RC stress and tableting stresses used. At low tableting stress the porosity increased with increasing MCC percentage in the mixture. 


\section{Chapter 4. LIXELL Particle Imaging and ${ }^{1} \mathbf{H}$ NMR}

\subsection{Introduction}

This chapter will explore modified methods to monitor tablet disintegration and dissolution. The standard USP disintegration analysis method (as described in Section 1.3) is only able to determine the disintegration time as a singular time point at the end of the process. No additional information is obtained regarding the processes which occur during the break-up of the tablet into smaller particles, which lead to the fully disintegrated tablet.

Wilson et al. established a particle imaging analysis technique to monitor particle release during disintegration (Wilson et al., 2012). A standard USP dissolution bath was modified so that the disintegration media could be analysed during the disintegration process, with the addition of a commercially available particle characterisation equipment. The set-up is based on the disintegration medium being pumped through the QICPIC LIXELL particle sizing equipment in a closed system. By doing this the particle size and number can be obtained for the released particles in the disintegration medium.

The QICPIC LIXELL method will be used in this Chapter to monitor tablet disintegration as it is capable of extracting further information regarding the mechanisms occurring during tablet disintegration. Previously, the method was 
used to analyse tablets containing an unspecified pharmaceutical formulation (Wilson et al., 2012). The formulation was wet granulated and tableted to produce tablets with varying properties. In this research dry granulation will be used to granulate binary mixtures of a brittle and a ductile material.

In industry, USP tablet dissolution analysis is determined on-line by the utilisation of conductivity or UV spectroscopy, depending on the compound being analysed. While these measurements are able to determine the release with time, problems arise when multiple soluble and detectable compounds exist. In the case of tablet dissolution measurements via conductivity, it is not possible to guarantee that the conductivity is caused by a single component within a tablet. There may be two or more soluble conductive components or some impurities within the tablet which may cause inaccurate readings to be obtained regarding the dissolution of a specific compound. UV measurements are able to produce spectra which can be used to identify specific compounds within the tablet and determine the concentration in solution at a given time. However, if more than one compound is present within the tablet with similar spectra, then some overlap may occur, meaning that the identification of one compound becomes more difficult.

In this research ${ }^{1} \mathrm{H}$ NMR will be employed, in addition to the LIXELL particle analysis system, as an off-line dissolution analysis method. ${ }^{1} \mathrm{H}$ NMR is commonly used as a characterisation method to determine the structure of a compound. The technique relies on the protons in a compound imparting peaks that are specific to the positioning and the number of protons present. By analysing the chemical shifts of the peaks and the spin-spin couplings it is possible to determine the exact structure of a compound. This method is more accurate than the commonly used 
methods to measure tablet dissolution, as soluble compounds are able to be independently identified with confidence (Coombes et al., 2014, Wren et al., 2017).

By observing the particle release during disintegration with time in addition to the off-line dissolution analysis, it will be possible to monitor disintegration and dissolution simultaneously. It will be determined whether it is possible to detect the effect of variation in formulation and processing parameters on tablet disintegration and dissolution by using these methods.

\subsection{Materials}

The details of the placebo binary mixtures of mannitol and MCC which will be analysed in this Chapter are outlined and characterised in Section 3.2.

\subsection{Methods}

\subsubsection{Granulation and tableting}

The granulation and tableting methods which were used are outlined in Sections 3.3.2 and 3.3.6, respectively. Table 4.1 shows the processing parameters used to produce the tablets which were analysed in this Chapter. The roller compaction (RC) pressure used to granulate the material was either 20 or 100 bar. The tableting force used to compact the granular material into tablets was either 5 or $20 \mathrm{kN}$. 
Table 4.1 Formulations and processing conditions used to produce binary mixture granules with abbreviations.

\begin{tabular}{|c|cccc|}
\hline Abbreviations & $\begin{array}{c}\text { Mannitol } \\
(\%)\end{array}$ & MCC (\%) & $\begin{array}{c}\text { Roller compaction } \\
\text { pressure (bar) }\end{array}$ & $\begin{array}{c}\text { Tableting } \\
\text { force (kN) }\end{array}$ \\
\hline Man100:MCC0 & 100 & 0 & 20,100 & 5,20 \\
\hline Man75:MCC25 & 75 & 25 & 20,100 & 5,20 \\
\hline Man25:MCC75 & 25 & 75 & 20,100 & 5,20 \\
\hline Man0:MCC100 & 0 & 100 & 20,100 & 5,20 \\
\hline
\end{tabular}

\subsubsection{LIXELL particle imaging disintegration analysis}

A conventional USP dissolution apparatus was linked in a circular loop system to a Sympatec QICPIC (Clausthal-Zellerfeld, Germany) particle size analysis apparatus (as shown in Figure 4.1). The QICPIC was fitted with the LIXELL liquid dispersion unit and a $1 \mathrm{~mm}$ width cuvette which the liquid medium flows through during analysis. The QICPIC LIXELL system is able to monitor the particle release during tablet disintegration. The images were analysed using Windox 5 software, and the data were analysed in terms of the cumulative number of released particles and mass weighted particle size distributions, including the $\mathrm{D}_{10}$ and $\mathrm{D}_{50}$ values, as functions of time.

$900 \mathrm{ml}$ of deionised water was used per experiment, which was maintained at $37{ }^{\circ} \mathrm{C}$ using a Clifton NE4-D thermostirrer (Weston-Super-Mare, UK) and water bath. A Heidolph RZR 2020 paddle stirrer (Schwabach, Germany) was used to agitate the liquid media using a $75 \mathrm{~mm}$ paddle at a speed of $56 \mathrm{rpm}$. Water was pumped around the disintegration set-up at a rate of approximately $10.6 \mathrm{ml} / \mathrm{s}$, using a Watson-Marlow 505Di peristaltic pump (Cornwall, UK). The particle number and 
sizes were measured for $30 \mathrm{sec}$ and the average of those values was recorded. The total run time was $10 \mathrm{~min}$ for each experiment. 2 tablets were tested for each

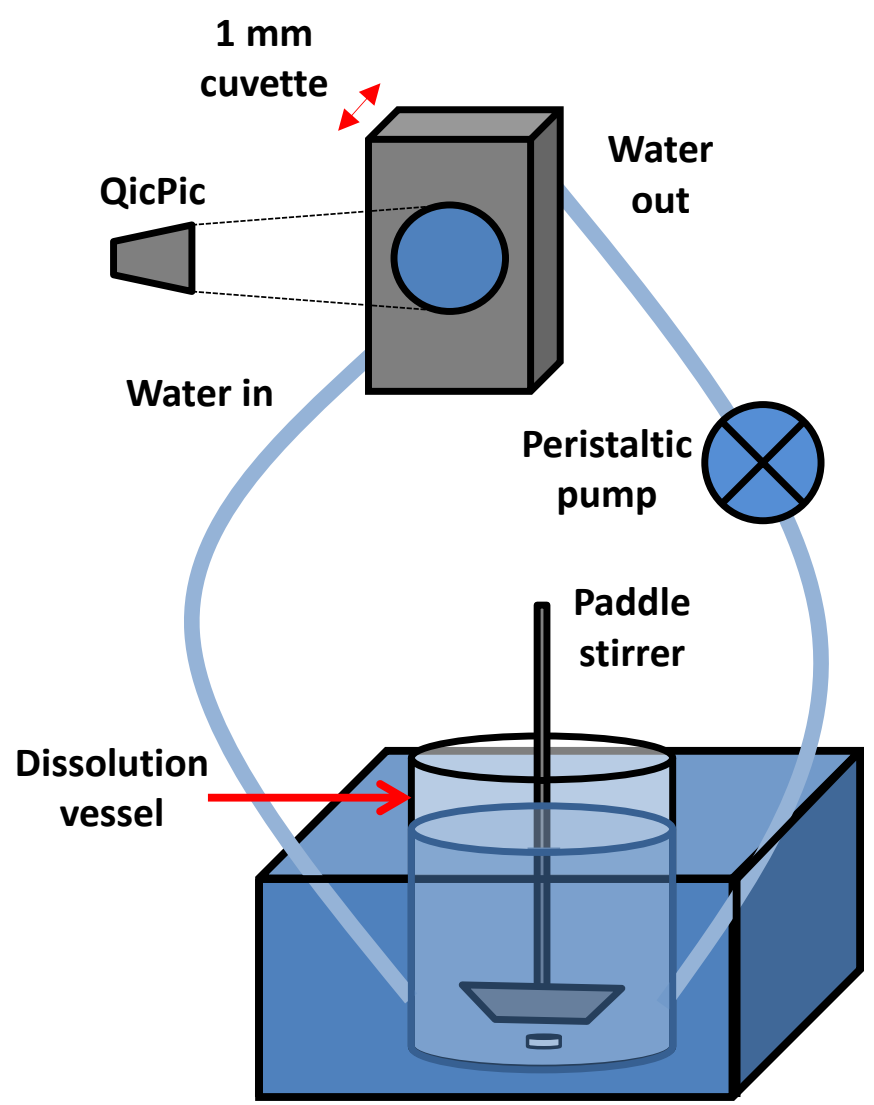

Heated water bath

condition, and the average values are presented.

Figure 4.1 Schematic of USP LIXELL setup.

It should be noted that the tablet undergoes two significant mechanical stresses during LIXELL disintegration analysis. Firstly, the paddle stirrer agitates the liquid and may cause breakage of the tablet. Secondly, the pump which circulates the liquid may cause further breakage of the tablet and released particles. 


\subsection{3 ${ }^{1} \mathrm{H}$ NMR dissolution analysis}

Tablet dissolution was determined by simultaneously removing samples during the LIXELL analysis outlined in Section 4.3.2. These samples were analysed off-line through ${ }^{1} \mathrm{H}$ NMR analysis on the liquid media. ${ }^{1} \mathrm{H}$ NMR was used at it is a characterisation method which is able to distinguish individual compound concentrations within a solution (Coombes et al., 2014, Wren et al., 2017). Existing methods for dissolution analysis, such as conductivity and UV spectroscopy are not as precise in determining individual components when multiple soluble components are present, i.e. in a pharmaceutical tablet formulation. In this research the dissolution percentage of mannitol will be determined for the various formulations.

Samples of $700 \mu \mathrm{l}$ for the analysis were removed at varying intervals and placed into a $5 \mathrm{~mm}$ NMR tube. An initial sample was taken at $\mathrm{t}=0 \mathrm{sec}$ which was used as a baseline reading. NMR analysis was carried out using a Bruker Biospin $600 \mathrm{MHz}$ spectrometer (Rheinstetten, Germany) equipped with a $5 \mathrm{~mm}$ TCI cryoprobe. ${ }^{1} \mathrm{H}$ NMR spectra were acquired at $26.85{ }^{\circ} \mathrm{C}(300 \mathrm{~K})$ without sample rotation and with two prior dummy scans. Twenty thousand points were acquired with a spectral width of $10.6 \mathrm{kHz}$, constant receiver gain, an acquisition time of $1 \mathrm{~s}$, and a recycle delay of $1 \mathrm{~s}$. To ensure high sensitivity 32 scans were run for a total experiment time of $70 \mathrm{~s}$. Water suppression was achieved using the NOESYpresaturation pulse sequence (Bruker noesygpprld pulse sequence) with irradiation at the water frequency during the recycle and missing time delays. Data were processed in Bruker Topspin 2.1 software. Exponential line broadening was applied with the LB parameter set to 0.19 , baseline correction was applied using the 
command "ABS" and integrals were directly compared across multiple spectra using the "Use lastscale for calibration" functionality.

\subsection{Results and Discussion}

\subsubsection{LIXELL}

The LIXELL-QICPIC system is a liquid particle imaging technique which is able to determine the size and number of particles in solution. In this case the disintegration medium was circulated into the LIXELL equipment and the size and number of particles in solution was determined with time. This was done in an attempt to better understand the disintegration process depending on the formulation used and the stresses applied during RC and tableting.

\section{Released particle size during disintegration}

The average volume weighted $\mathrm{D}_{10}$ and $\mathrm{D}_{50}$ values for the various binary tablets were obtained from the QICPIC LIXELL measurement and are shown in Figure 4.2. It should be noted that the margin of error is relatively high for these data points, so this trend cannot be confirmed with confidence.

Firstly, it was observed that Man100:MCC0 tablets released the largest particles during tablet disintegration (see Figure 4.2). Mannitol is a soluble material which was easily dispersed into the disintegration media. Additionally, MCC is a disintegrant, meaning that the material draws water into the tablet which causes it to break into smaller fragments. Therefore, the Man100:MCC0 tablets in the absence of MCC do not disintegrate as efficiently into smaller particles as the tablets which contain MCC. This phenomenon may also be influenced by mannitol primary powder being larger in size (see Table 3.1). The particle release depending on the RC pressure or tableting force used is not clear due to the high error. 


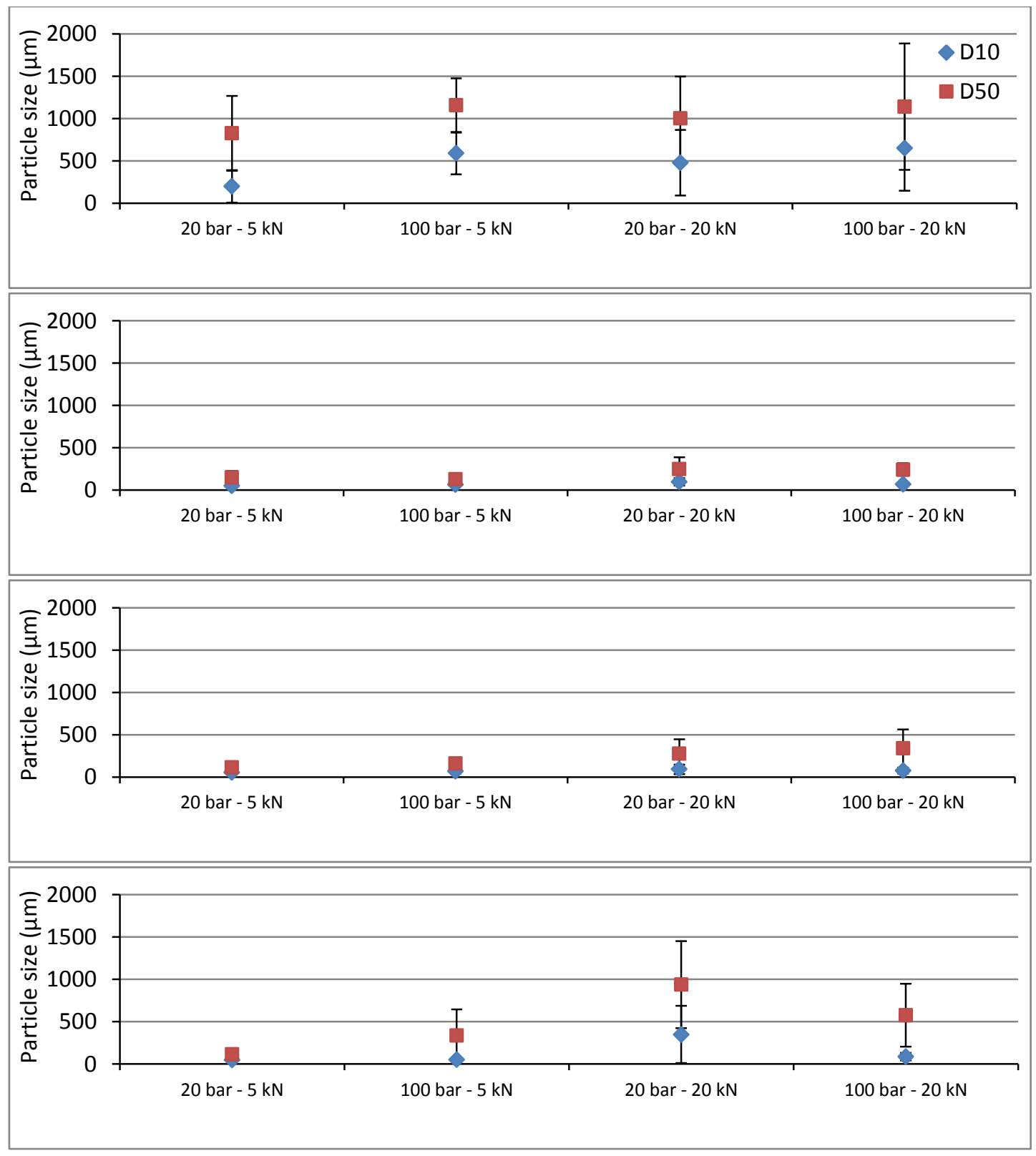

Figure 4.2 Average $D_{10}$ and $D_{50}$ values for tablets produced using varying formulations and processing parameters. Observed using the LIXELL-QICPIC system.

Tablets which contain a mixture of mannitol and MCC tended to release much smaller particles than Man100:MCC0 tablets (see Figure 4.2). The particle release did not vary significantly depending on the processing parameters; again the error is too high. 
For Man0:MCC100 tablets, the size of the released particles were higher for tablets which were compressed using $20 \mathrm{kN}$ tableting force. Visually, during the measurements it was observed that these particular Man0:MCC100 tablets absorbed water and in the case of 20 bar RC pressure the tablet swelled and disintegrated into very large fragments (see Figure 4.3). In the case of 100 bar RC pressure the tablet disintegrated into relatively smaller fragments. In both cases the tablets disintegrated into larger fragments, and tended to settle at the bottom of the dissolution vessel (see Figure 4.3 and Figure 4.4).

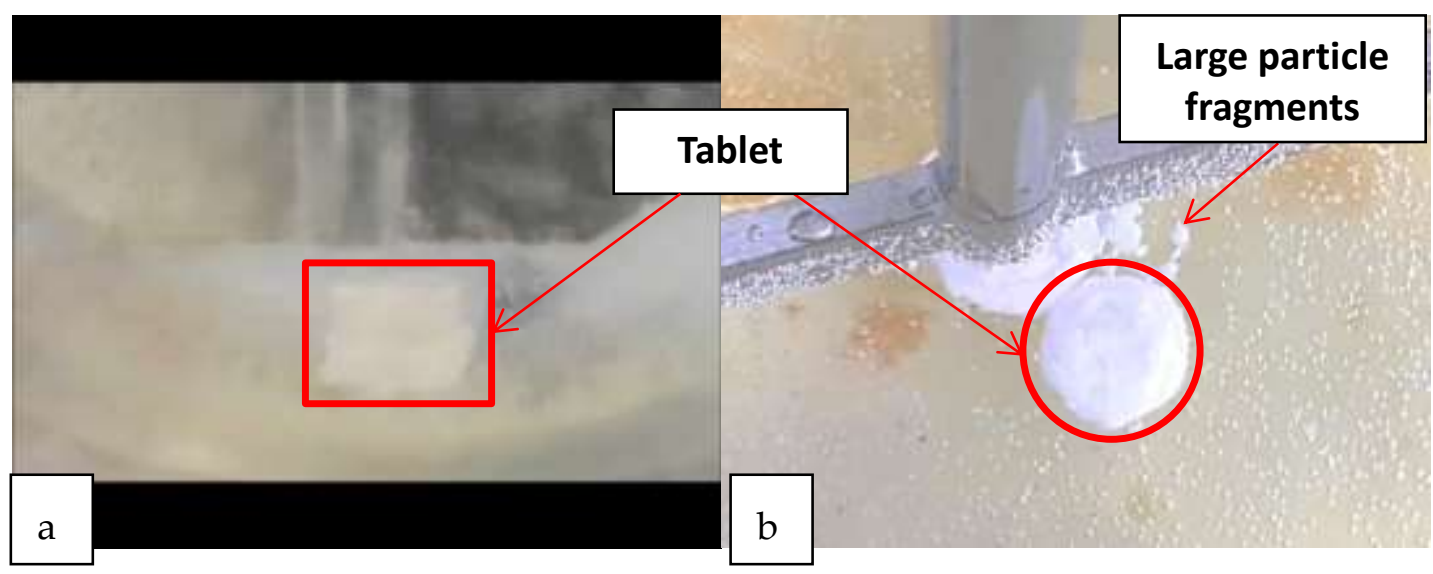

Figure 4.3 Man0:MCC100 tablet produced using 20 bar granules compacted using $20 \mathrm{kN}$ tableting force a) during disintegration at the maximum swelled size and b) after the disintegration measurement ended. 


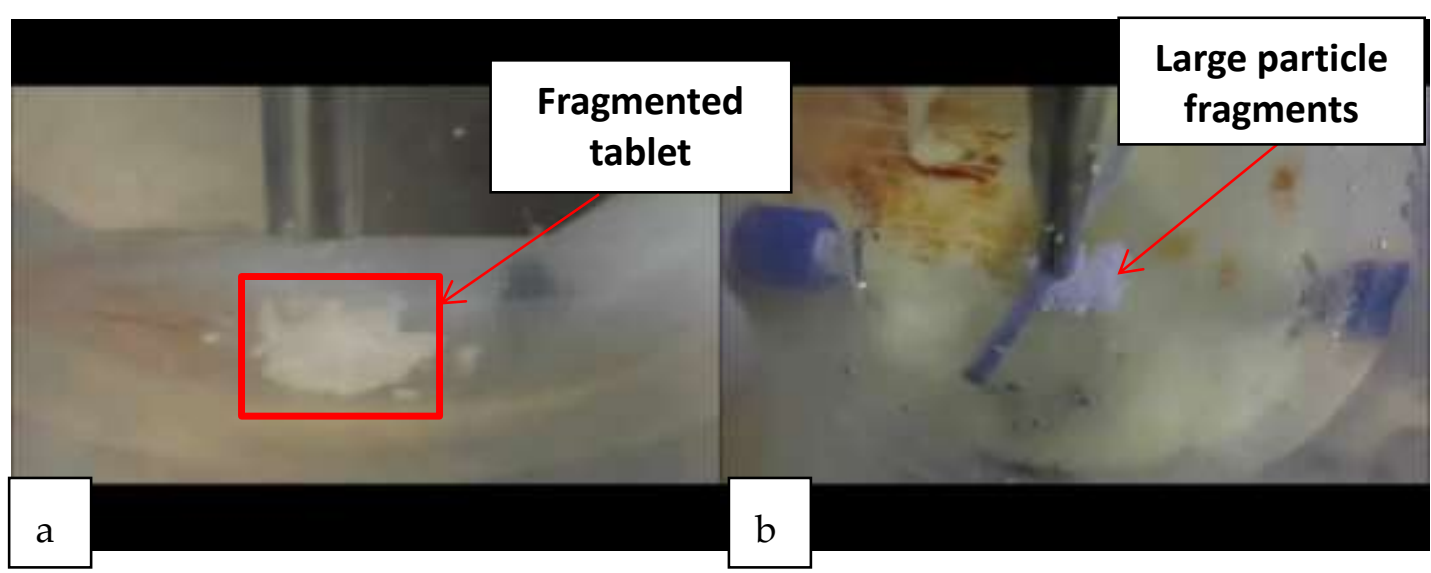

Figure 4.4 Man0:MCC100 tablet produced using 100 bar granules compacted using $20 \mathrm{kN}$ tableting force a) during disintegration at the maximum swelled size and b) after the disintegration measurement ended.

Figure 4.5 shows the $\mathrm{D}_{10}$ and $\mathrm{D}_{50}$ values with time for particles that were released from Man100:MCC0 tablets, which were obtained using the LIXELL system. The tablets were produced using 20 and 100 bar RC pressure and 5 and 20 $\mathrm{kN}$ tableting force. It should be noted that the $\mathrm{D}_{10}$ and $\mathrm{D}_{50}$ values are significantly scattered and the error is substantial. Therefore, the trends which may exist cannot be accepted with high certainty.

Overall, the particle sizes for Man100:MCC0 fluctuated drastically throughout the disintegration and the error was high. The number of entrained particles, which will be examined in the next Section, may provide additional information as to what is occurring. The particle size distributions with time are shown in Appendix 1 Appendix 16. 


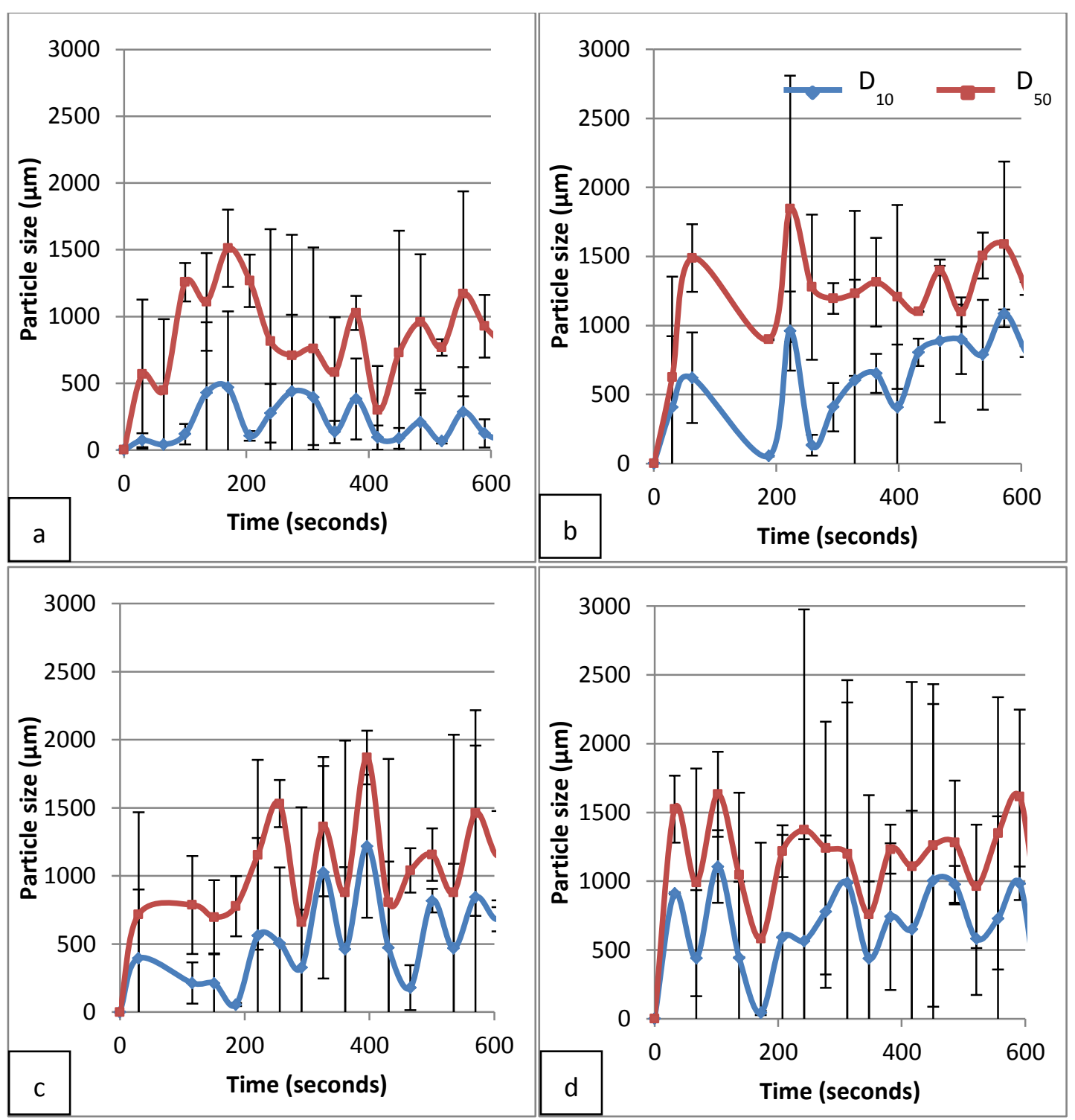

Figure 4.5 The change in $\mathrm{D}_{10}$ and $\mathrm{D}_{50}$ values with time for Man100:MCC0 tablets produced using a) 20 bar RC pressure, $5 \mathrm{kN}$ tableting force; b) 100 bar RC pressure, $5 \mathrm{kN}$ tableting force; c) 20 bar RC pressure, $20 \mathrm{kN}$ tableting force; d) $100 \mathrm{bar}$ RC pressure, $20 \mathrm{kN}$ tableting force.

Figure 4.6 shows the $\mathrm{D}_{10}$ and $\mathrm{D}_{50}$ particle size data for Man75:MCC25 tablets produced using varying RC pressure and tableting stresses. Much smaller sized particles were released from this formulation compared to Man100:MCC0 tablets. It 
is possible that larger particles were released initially for $20 \mathrm{kN}$ tablets (Figure 4.6c and Figure 4.6d), however the error is very high to validate this.

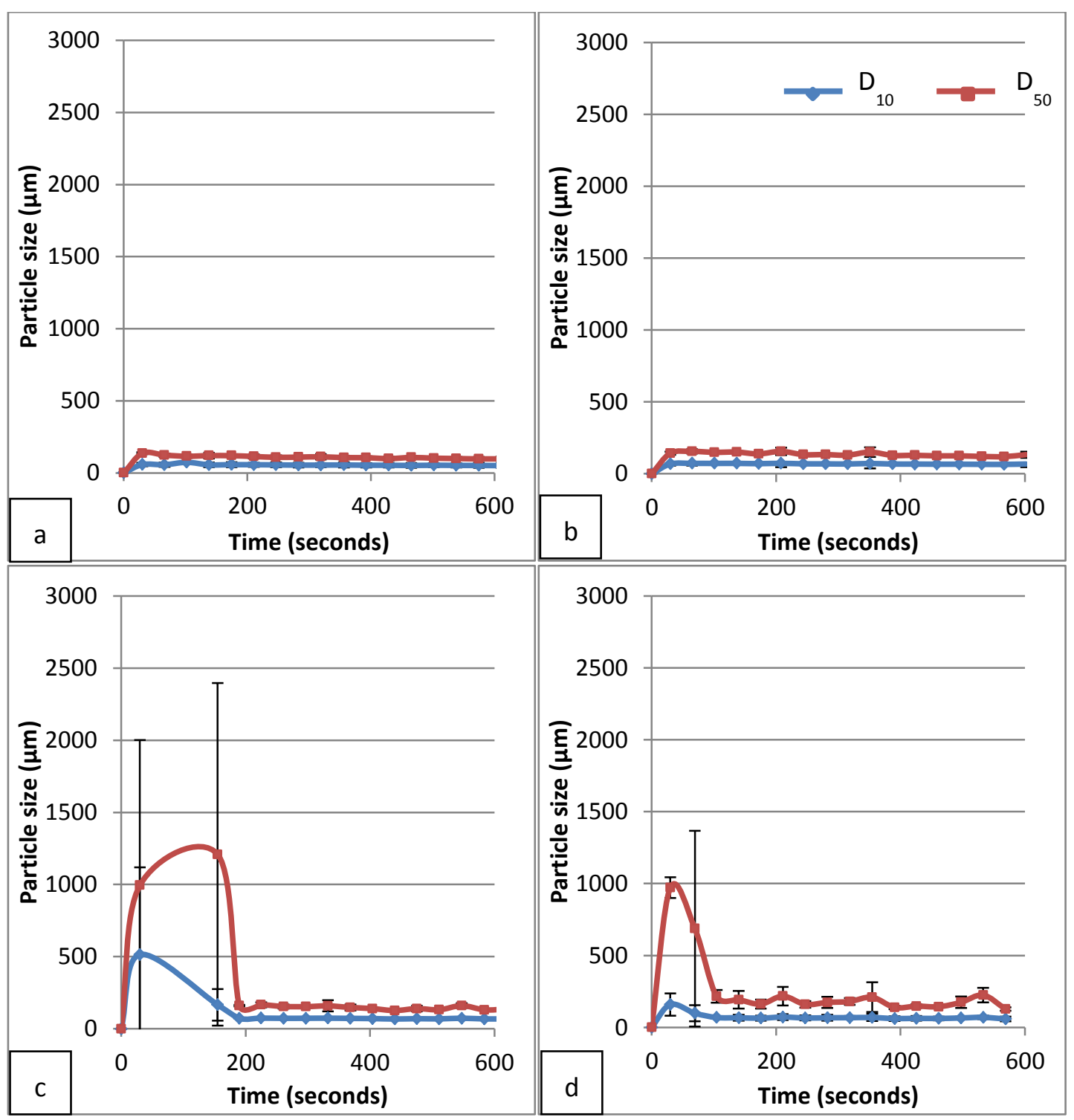

Figure 4.6 The change in $D_{10}$ and $D_{50}$ values with time for Man75:MCC25 tablets produced using a) 20 bar RC pressure, $5 \mathrm{kN}$ tableting force; b) 100 bar RC pressure, $5 \mathrm{kN}$ tableting force; c) $20 \mathrm{bar}$ RC pressure, $20 \mathrm{kN}$ tableting force; d) $100 \mathrm{bar}$ RC pressure, $20 \mathrm{kN}$ tableting force.

Figure 4.7 shows the released particle sizes for Man25:MCC75 tablets. The tablets formed using low tableting force released the small particles (see Figure 4.7a 
and Figure 4.7b). A higher tableting force released slightly larger particles throughout the disintegration process (see Figure 4.7c and Figure 4.7d). However, the error is significant for this data.

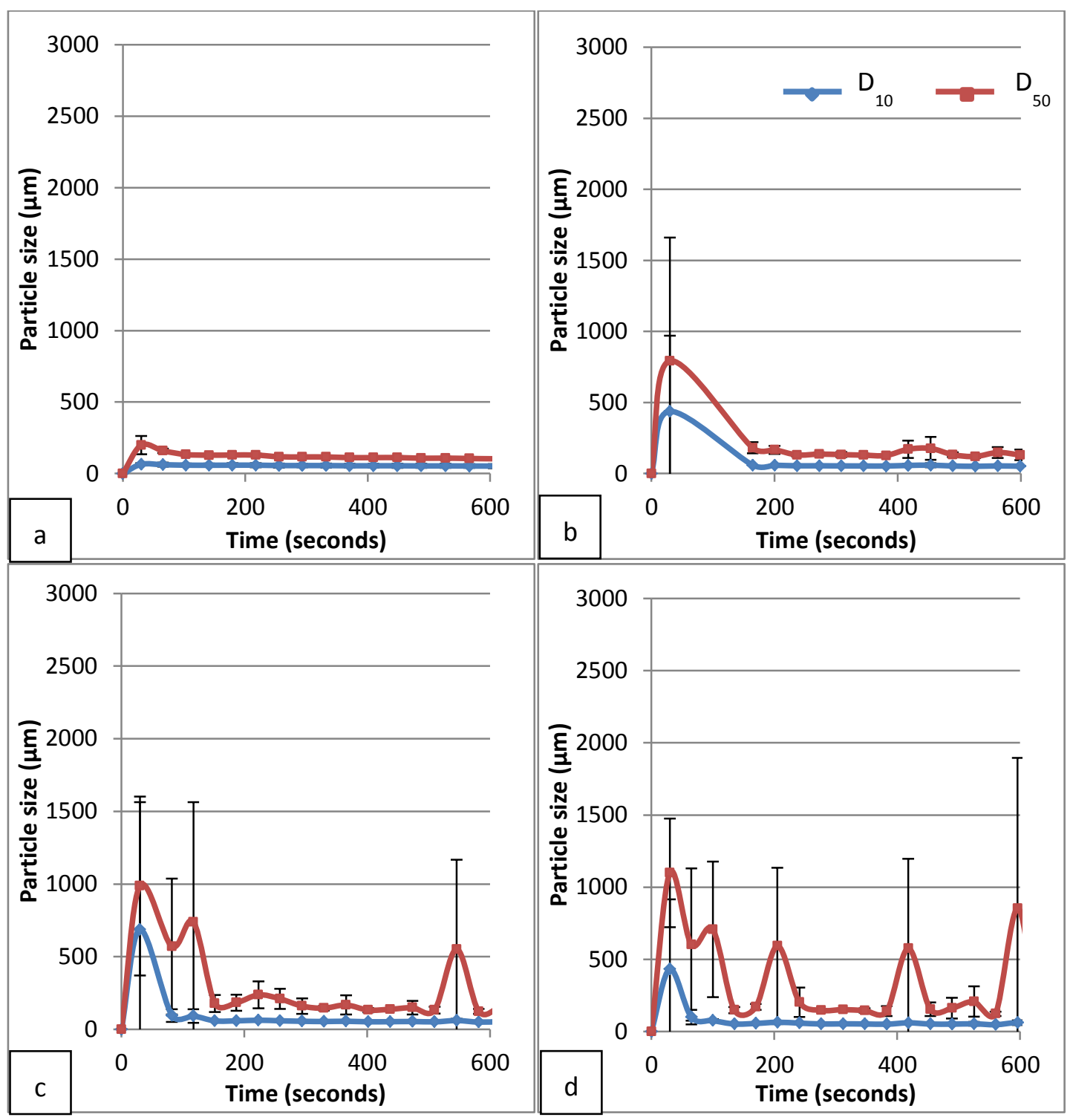

Figure 4.7 The change in $D_{10}$ and $D_{50}$ values with time for Man25:MCC75 tablets produced using a) 20 bar RC pressure, $5 \mathrm{kN}$ tableting force; b) 100 bar RC pressure, $5 \mathrm{kN}$ tableting force; c) $20 \mathrm{bar}$ RC pressure, $20 \mathrm{kN}$ tableting force; d) $100 \mathrm{bar}$ RC pressure, $20 \mathrm{kN}$ tableting force. 
Figure 4.8 shows particle release data for Man0:MCC100 tablets. It should be noted that a significant percentage of the MCC fragments were unable to be pumped into the LIXELL cuvette for analysis. The biggest tablet fragments settled at the bottom of the USP vessel which is a phenomenon that is termed "coning" (see Figure 4.3 and Figure 4.4). Therefore, it should be taken in to consideration that not all $100 \%$ of the released particles were able to be analysed by the LIXELL method.

Application of low RC pressure and low tableting force leads to the tablet disintegrating into the smallest size particles (Figure 4.8a). A high RC pressure and a low tableting force led to the release of larger particles which fluctuated in size throughout disintegration (Figure 4.8b). When a higher tableting stress was used the tablets released particles of a much larger size (Figure $4.8 \mathrm{c}$ and Figure $4.8 \mathrm{~d}$ ) than when a low stress was applied. The tablets produced using low RC pressure granules and high tableting stress released the largest particles. But the significant error in these results must be considered.

It was observed that the released particles were large in size throughout the disintegration process for Man0:MCC100 tablets. This is likely due to MCC being insoluble in water. Overall, Man100:MCC0 tablets were found to release much larger particles for every processing parameter. The next Section will explore the number of particles released which may offer some explanation for this phenomenon. 


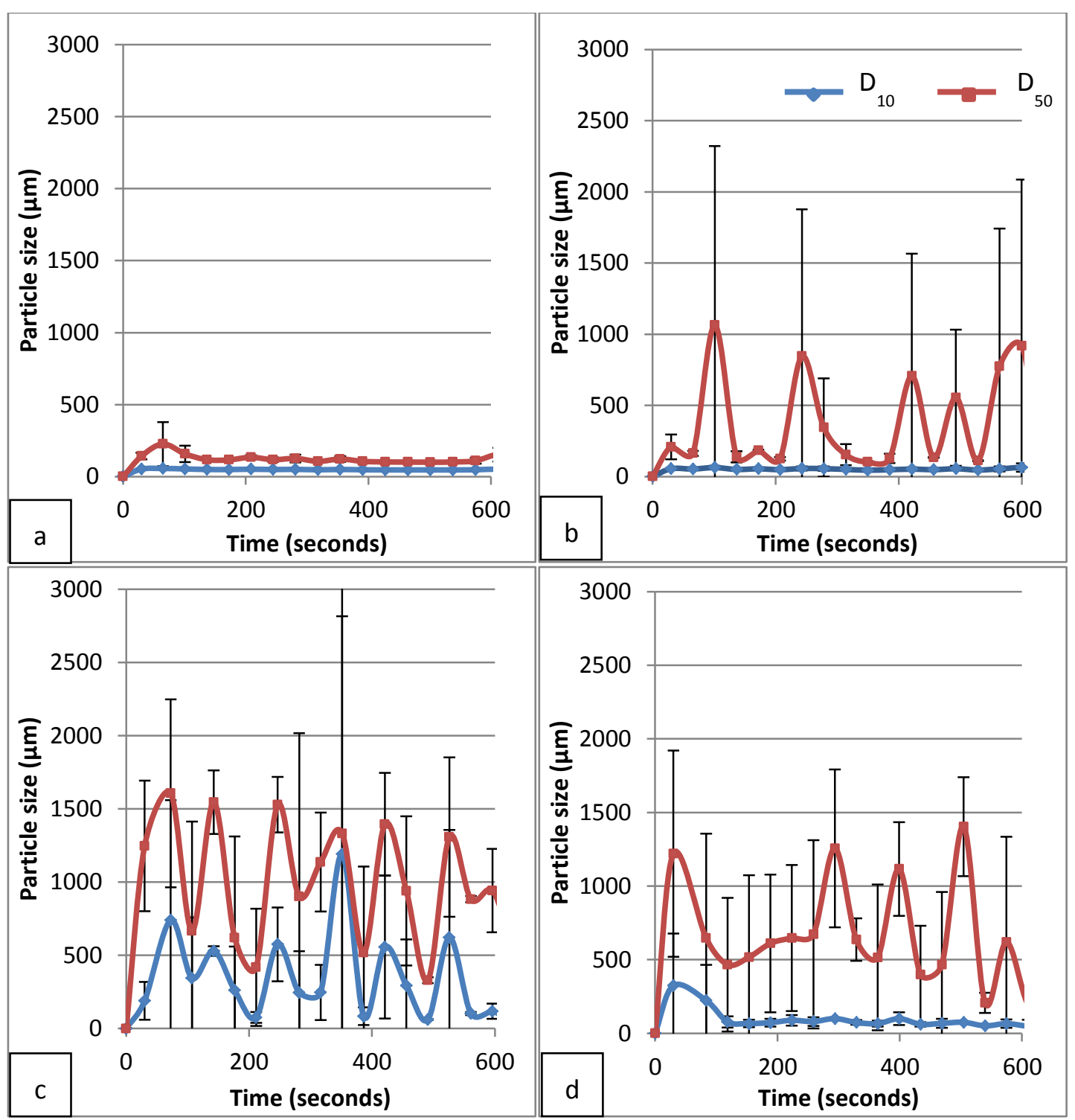

Figure 4.8 The change in $D_{10}$ and $D_{50}$ values with time for Man0:MCC100 tablets produced using a) 20 bar RC pressure, $5 \mathrm{kN}$ tableting force; b) 100 bar RC pressure, $5 \mathrm{kN}$ tableting force; c) 20 bar RC pressure, $20 \mathrm{kN}$ tableting force; d) $100 \mathrm{bar}$ RC pressure, $20 \mathrm{kN}$ tableting force.

\section{Number of entrained particles}

The numbers of entrained particles, i.e. the number of particles released from the tablet which exist in the disintegration medium at a given time, were examined. 
The numbers of entrained particles were detected for each tablet condition by pumping the disintegration media into the QICPIC particle imaging system.

Figure 4.9 shows the number of entrained particles with time for Man100:MCC0 tablets produced using 20 and 100 bar RC pressure, with 5 and 20 $\mathrm{kN}$ tableting forces. From Figure 4.5 it was observed that generally larger particles were released throughout the disintegration process. By looking at the number of particles in Figure 4.9 it can be seen that while the size of the particles were larger, the number is significantly lower than the other tablet formulations. This is undoubtedly due to mannitol being a soluble material therefore the number of particles observed is considerably low, as the material quickly dissolves into solution.

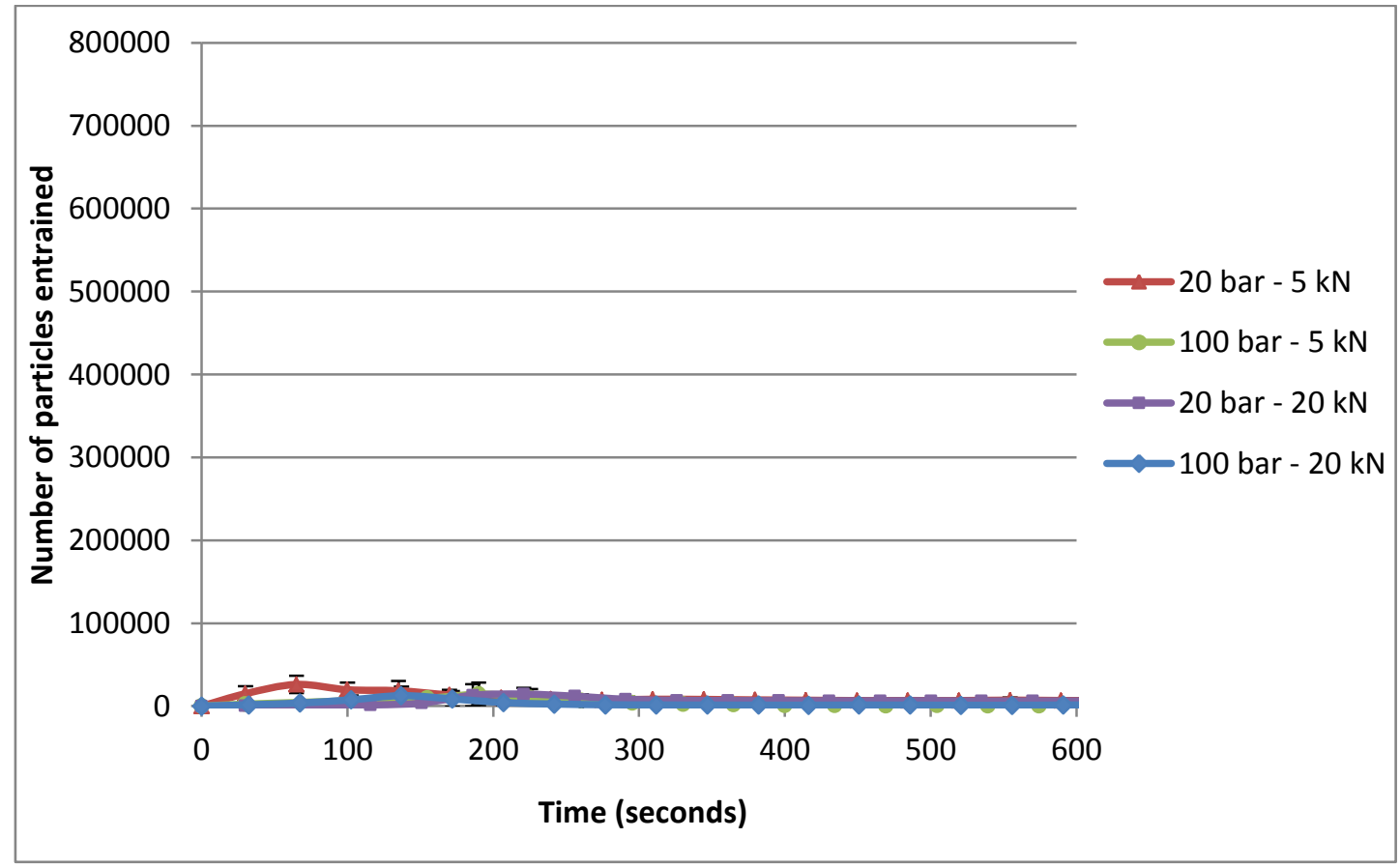

Figure 4.9 Number of entrained particles for Man100:MCC0 tablets produced using varying processing parameters. 
Figure 4.10 shows the number of entrained particles with time for tablets the containing Man75:Man25 formulation. Firstly, it should be noted that tablets which contain any percentage of MCC release much a much higher total number of particles than tablets which do not contain MCC. For tablets which contain MCC the number of entrained particles increases initially at a fast rate, then a secondary stage of particle release is observed where the number of entrained particles continues to increase, albeit at a slower rate than exhibited at the initial stage.

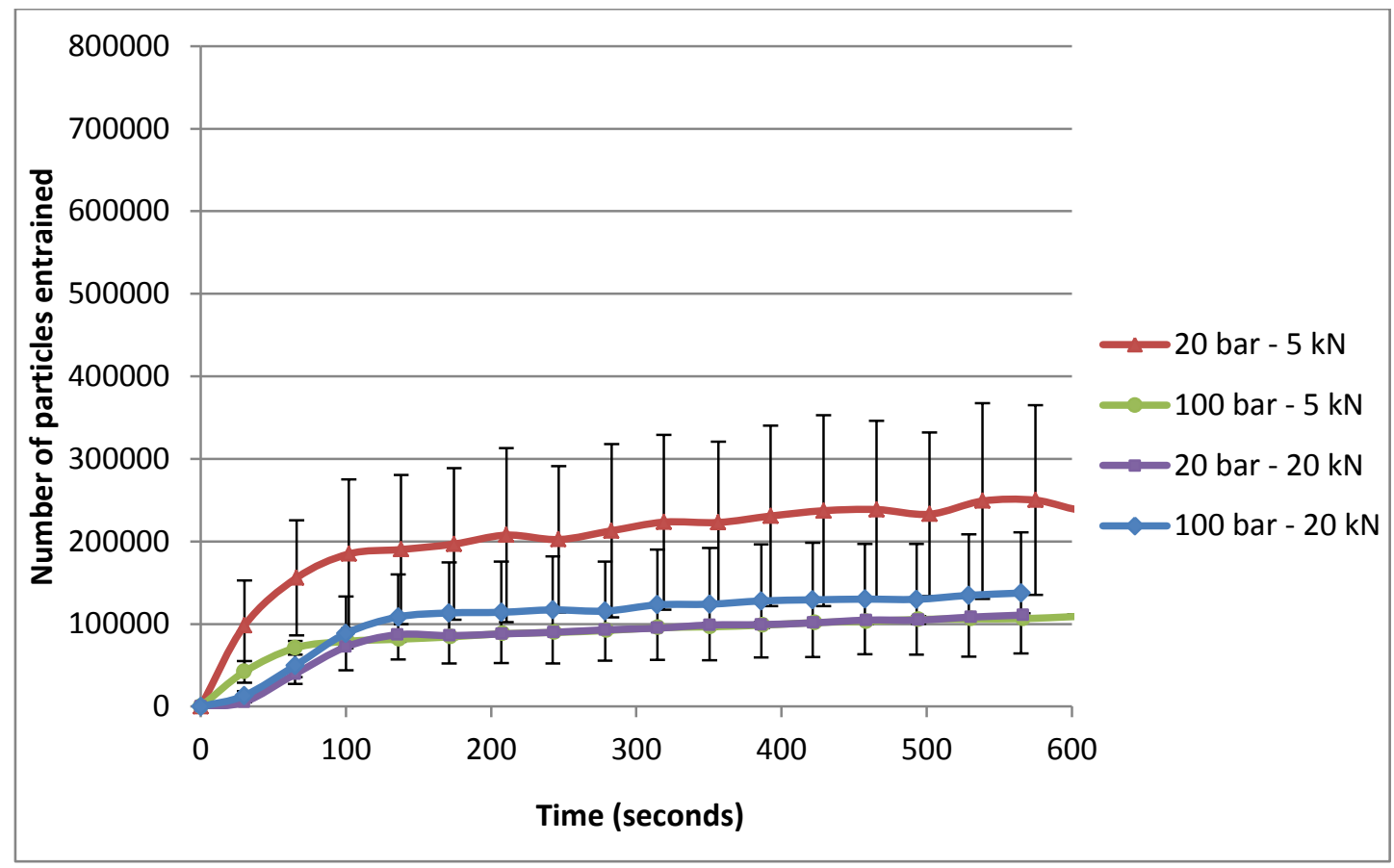

Figure 4.10 Number of entrained particles for Man75:MCC25 tablets produced using varying processing parameters.

The 20 bar RC pressure granules and a low tableting force led to the rapid release of a high number of particles during the initial stage. From the compactibility analysis (see Figure 3.11) it was determined that these tablets were the most porous therefore the rapid disintegration is expected as the water penetration into the tablets is also rapid. Subsequently, during the secondary stage 
after approx. $100 \mathrm{~s}$ the number of entrained particles continues to increase, however, at a slower rate than the initial stage. The number of entrained particles continues to increase, to the highest total value of all of the tablets and at the highest rate, until the experiment was terminated. From the compactibility data it was found that the tablets are porous and weak (see Section 3.4.3). And from X-ray analysis it was determined that the granular material was crushed into smaller particles (see Section 3.4.4). Therefore, it can be assumed that weakly compressed small particles exist within the tablet, which supports the particle release behaviour observed. For the other tablets, the error is too high to comment on any particle release trends.

Figure 4.11 shows the number of entrained particles with time for Man25:MCC75 tablets, which were produced using varying RC and tableting stresses. Following the same trend as the Man75:MCC25 formulation, tablets produced using 20 bar RC pressure and $5 \mathrm{kN}$ tableting force initially had a rapid increase in the number of entrained. Following this at approximately $100 \mathrm{~s}$ the number of entrained particles continues to increase, to the highest value for this formulation of approximately 500000 particles, but at a slightly slower rate than at the initial stage. For all other tablets in this formulation group, the number of entrained particles increased steadily throughout the measurement.

20 bar tablets produced using a low tableting force release a higher number of smaller particles (see Figure 4.7a and Figure 4.11). Section 3.4.4 established that low $\mathrm{RC}$ pressure tends to be crushed into smaller particles during the tableting process, as the granular material is weaker than when a high RC pressure is used. Therefore, during tableting the low force applied was sufficient to break the granular material. However from the compactibility graphs (see Section 3.4.3) it can be seen that the tableting force is inadequate to produce strong bonds, and therefore the tablet is 
weaker. Consequently, during the LIXELL analysis the tablet disintegrates rapidly due to the tablet being weak and porous. And in conclusion the tablet breaks up into a higher number of smaller particles.

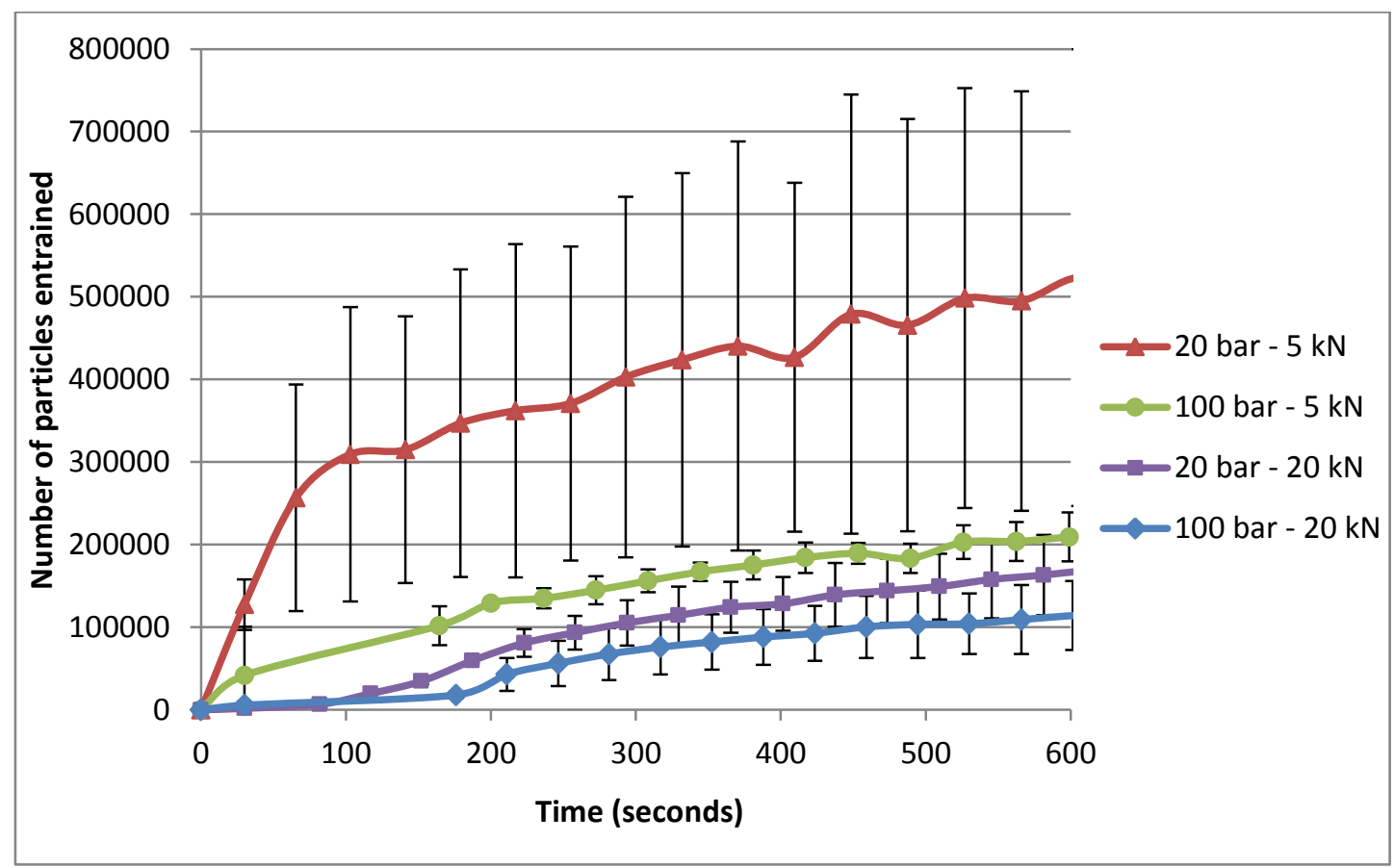

Figure 4.11 Number of entrained particles for Man25:MCC75 tablets produced using varying processing parameters.

Tablets produced using 100 bar RC pressure and a low tableting force exhibited a slower increase in the total number of entrained particles compared to the 20 bar tablets. From Figure 4.7 it was observed that 100 bar tablets release slightly larger particles. From Section 3.4.4 it was found that a low tableting force was insufficient to crush the granular material. Therefore, it can be concluded that the tablet releases a smaller number of larger particles, i.e. granular material, than compared with 20 bar tablets.

Figure 4.12 shows the entrained particles with time for Man0:MCC100 tablets. Overall, a lower number of particles than expected were observed. For tablets 
containing a mixture of mannitol and MCC, a higher percentage of MCC led to a higher number of particles. This is due to the percentage of insoluble material increasing, therefore, more particles were observed. However, for tablets containing only MCC, the number of particles observed was relatively lower. This is due to the MCC particles becoming saturated with water and settling at the bottom of the disintegration vessel, meaning that a significant percentage of the tablet material is unable to be detected. This was especially the case for tablets produced using a high tableting force (see Figure 4.3 and Figure 4.4).

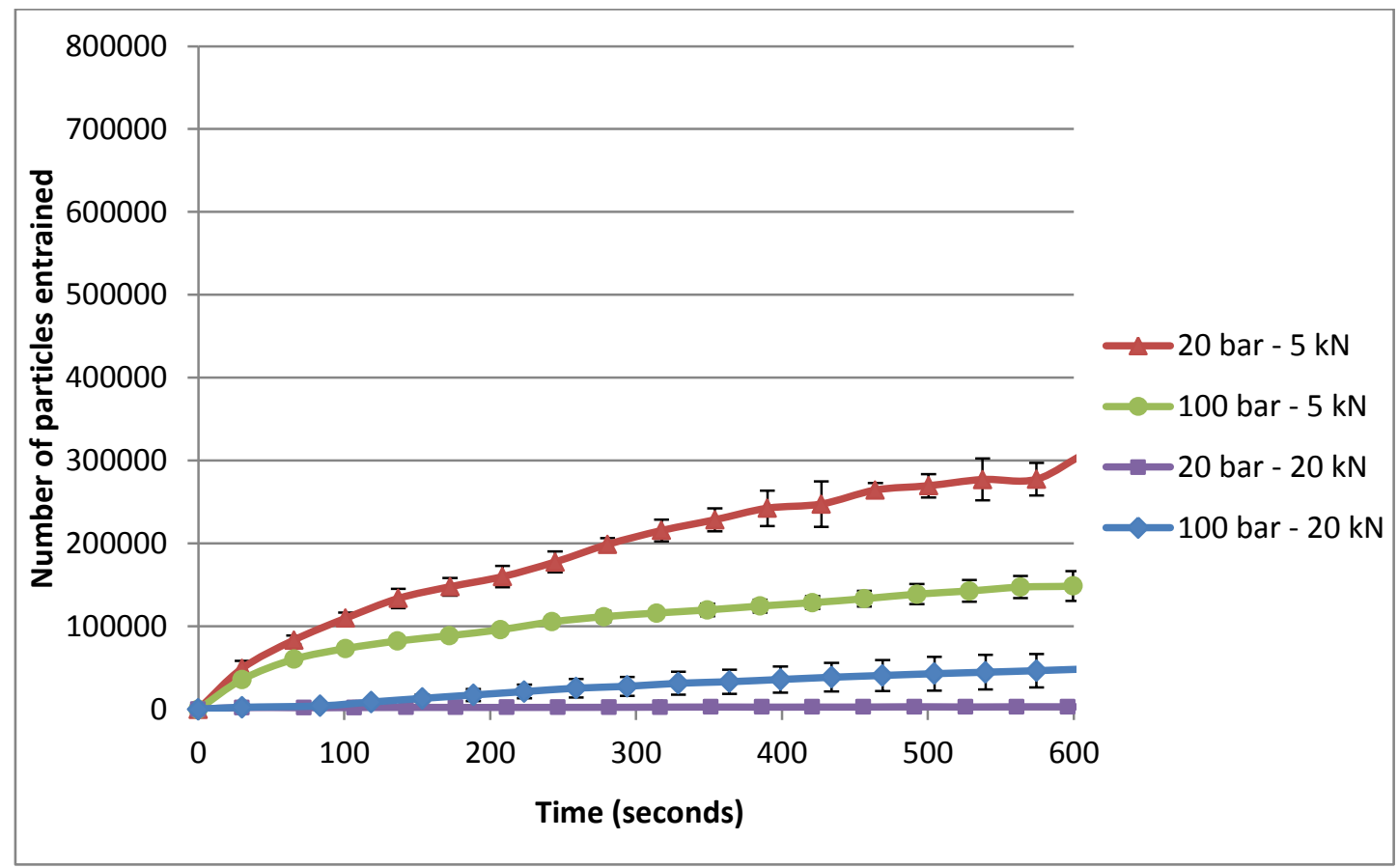

Figure 4.12 Number of entrained particles for Man0:MCC100 tablets produced using varying processing parameters.

Visually, 20 bar granules compacted using a high force produced tablets which behaved differently during the disintegration process. The tablets appeared to absorb water quickly and expanded vertically. The tablet swelled to its maximum size by approximately $1 \mathrm{~min}$ (see Figure 4.3). However the majority of the material 
did not readily break into smaller fragments with time and coning was prevalent (see Figure 4.3). This explains why such a low number of entrained particles were observed for these tablets. Tablets produced using 100 bar granules and a high tableting force also swelled vertically with time; however they also readily broke in to large fragments (see Figure 4.4). But due to the fragments settling in the vessel, a low number of entrained particles were detected.

The tablets produced using a lower tableting force exhibited similar behaviour to the previous formulations; a lower RC pressure led to the rapid release of a high number of particles. Tablets containing 100 bar granules released a lower number of particles than 20 bar tablets. The 20 bar particles are possibly smaller in size than the 100 bar tablets (see Figure 4.8a and Figure 4.8b) due to the granule particles being weakly bonded together.

\subsection{2 ${ }^{1} \mathrm{H}$ NMR dissolution analysis}

Samples were removed from the USP dissolution beaker and analysed using ${ }^{1} \mathrm{H}$ NMR to determine the relative concentration of mannitol in the solution. Man100:MCC0, Man75:MCC25 and Man25:MCC75 tablets produced using varying granulation and tableting stresses were analysed. Samples were taken for the NMR measurements at varying time intervals and analysed off-line. It must be taken into account that as this is an off-line measurement, the time from when the sample was taken from the disintegration measurement to the time when the NMR measurement is carried out allows for the dissolution of any particles which may exist in the solution. So for a measurement taken at a given time, the relative mannitol percentage determined by the NMR is an indication of the particles of mannitol which have already dissolved at that time, as well as any undissolved 
particles which would be detected by the QICPIC LIXELL system at the same time during the disintegration measurement.

Figure 4.13 shows the mannitol release profile for Man100:MCC0 tablets. The NMR dissolution profile shows that in most cases the mannitol has fully dissolved by $300 \mathrm{~s}$. When comparing the NMR dissolution profile and the number of particles released (see Figure 4.9), such low overall numbers were released by Man100:MCC0 tablets that this cannot be related back to the dissolution trends.

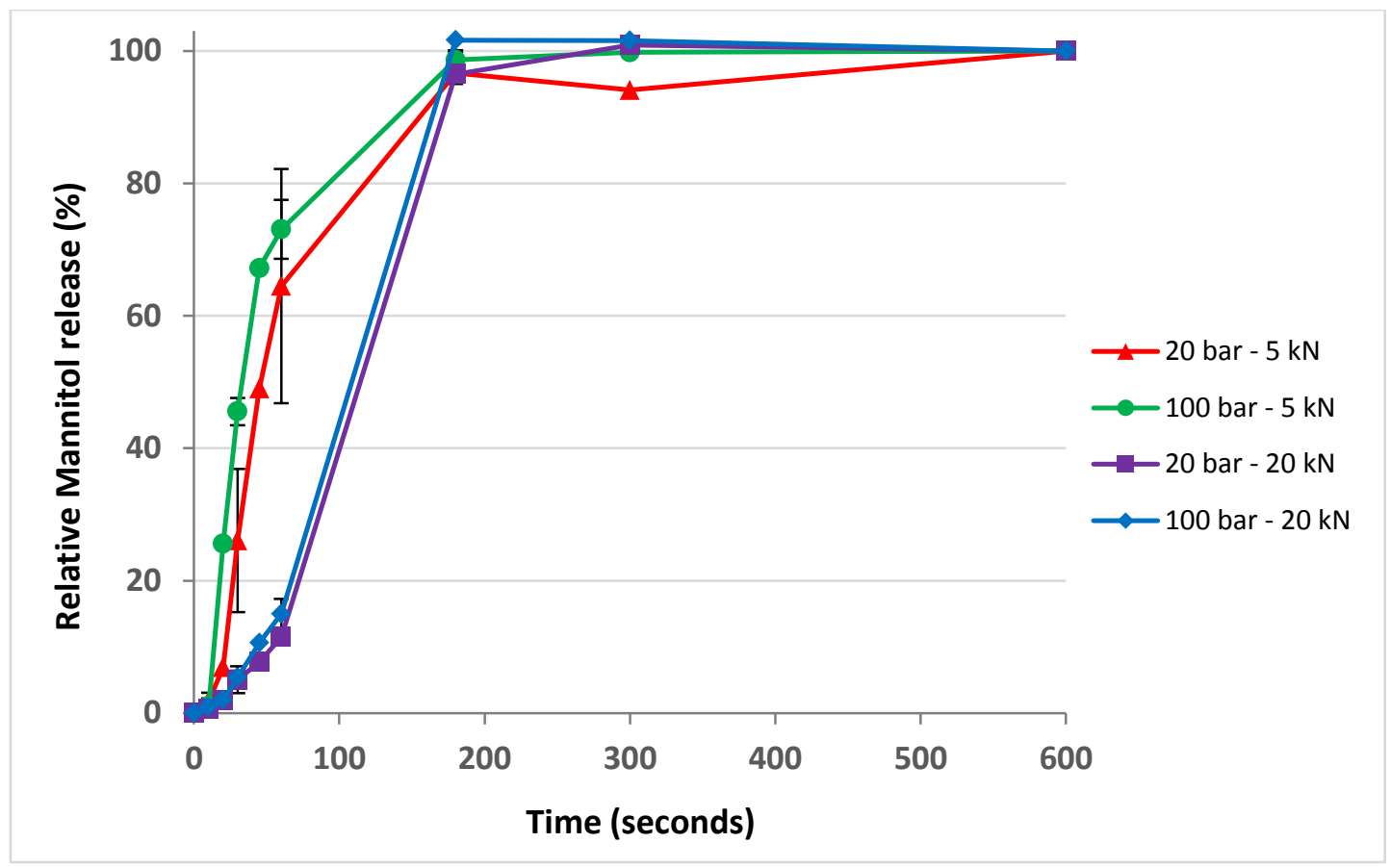

Figure 4.13 Relative mannitol release profile for Man100:MCC0 tablets, produced using various processing parameters, measured using ${ }^{1} \mathrm{H}$ NMR spectroscopy.

It can be clearly observed that when a higher tableting force was used, the rate of mannitol release was much slower than when a low tableting force was used. This is due to tablets produced using a low tableting force having a lower tensile strength and higher porosity (see Figure 3.11). This means that the water uptake is rapid and a higher number of particles are released during disintegration. A higher 
number of released particles present a higher surface area for the dissolution of mannitol into solution; therefore the dissolution is quicker for weaker and more porous tablets. The variation in the mannitol release depending on the roller compaction pressure used for a given tableting force is unclear; the error is too high.

Figure 4.14 shows the mannitol release profile for Man75:MCC25 tablets. The trend in the rate of release depending on the tableting force used is the same for Man100:MCC0 and Man75:MCC25 tablets. Tablets produced using a low tableting force released mannitol at a faster rate than when a high tableting force was used.

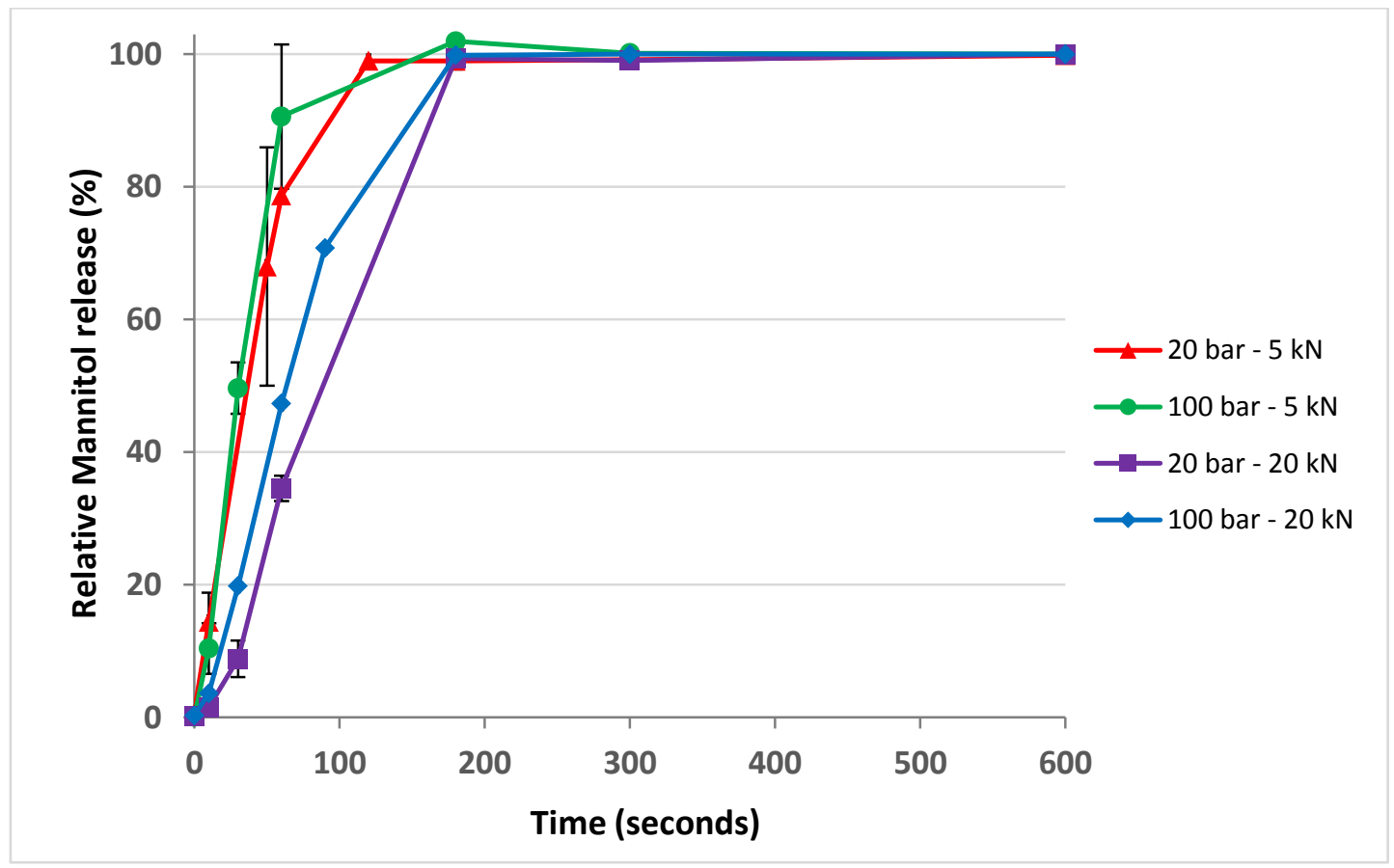

Figure 4.14 Relative mannitol release profile for Man75:MCC25 tablets, produced using various processing parameters, measured using ${ }^{1} \mathrm{H}$ NMR spectroscopy.

Figure 4.15 shows the release profiles for Man25:MCC75 tablets. For this formulation the mannitol release varied depending on the $\mathrm{RC}$ pressure and tableting force used. In this case the tablets containing 20 bar granules released mannitol at the fastest rate. From Figure 3.11 and Figure 3.12 it was determined that 
for Man25:MCC75 tablets the porosity as well as the tensile strength increases with increasing RC pressure used. The 20 bar granules tableted using $5 \mathrm{kN}$ force are stronger but also more porous than 100 bar granules. Therefore, it can be assumed that the influence of the low tablet porosity on the faster tablet disintegration is greater than tablet tensile strength. Tablets produced using 20 bar RC pressure and $20 \mathrm{kN}$ tableting force released mannitol at the slowest rate, due to the tablet being the strongest and the least porous.

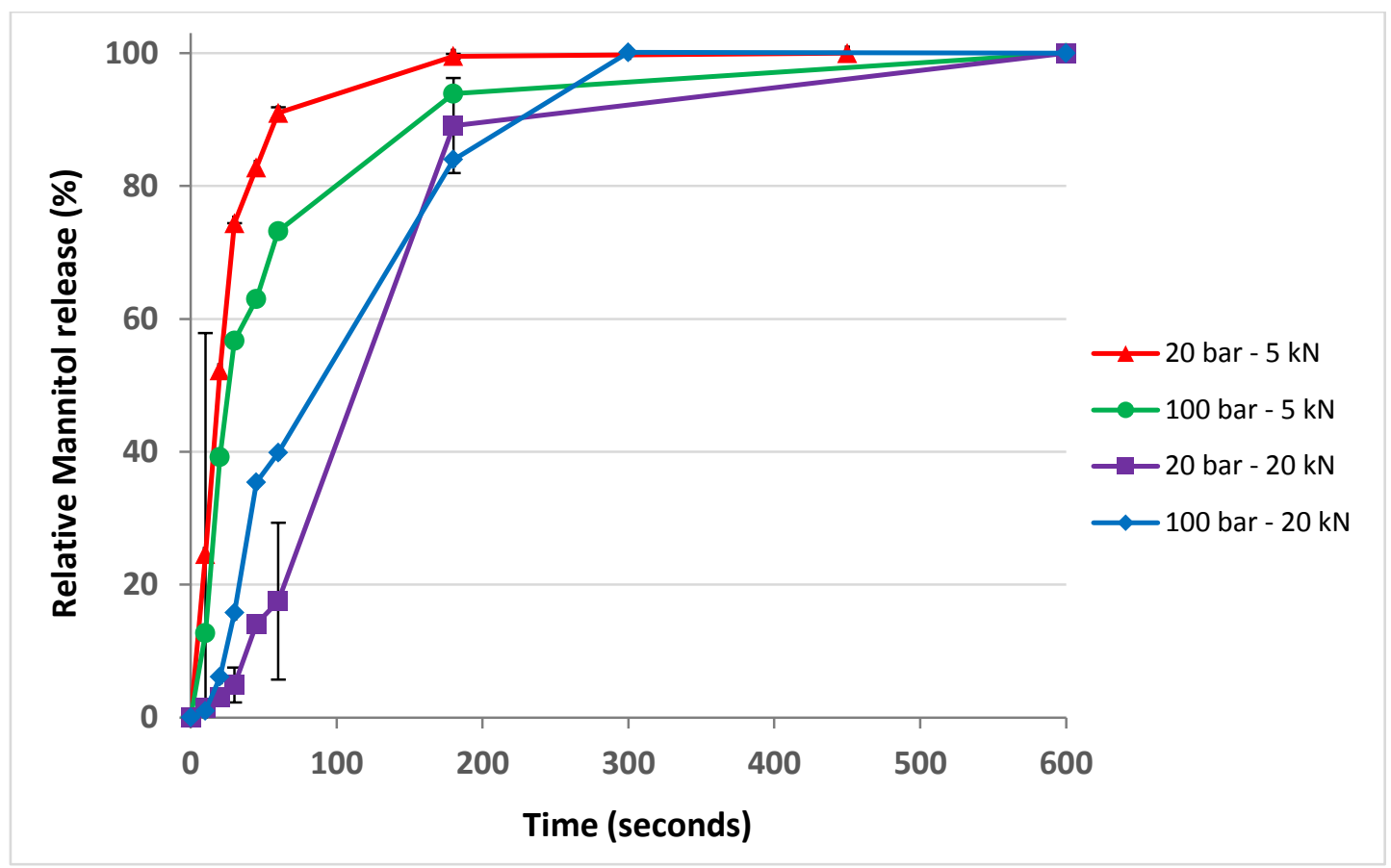

Figure 4.15 Relative mannitol release profile for Man25:MCC75 tablets, produced using various processing parameters, measured using ${ }^{1} \mathrm{H}$ NMR spectroscopy.

Figure 4.16 shows the relative mannitol release for Man100:MCC0, Man75:MCC25 and Man25:MCC75 tablets at $60 \mathrm{~s}$. The tablets were produced using 20 and $100 \mathrm{RC}$ pressure and 5 and $20 \mathrm{kN}$ tableting force. The relative percentage of mannitol in solution at $60 \mathrm{~s}$ has been plotted to determine the concentration of mannitol in solution depending on the formulation and processing conditions used. 
It was observed that tablets produced using a lower tableting load produced particles at a faster rate. This is likely due to these tablets being weaker and more porous. Therefore, water influx into the tablet is more rapid which encourages the dissolution process.

It was observed that for Man100:MCC0 tablets mannitol did not dissolve into solution at the fastest rate in any case, which is counter-intuitive. Appendix 17 indicates that the volume of Man100:MCC0 particles in solution is much higher than tablets containing MCC. This suggests that the mannitol is not readily dissolving, as the tablet consisting entirely of mannitol released a higher volume of solid particles. The LIXELL system observed a much lower volume of particles released from tablets containing MCC which suggests more of the soluble material dissolved into solution, as confirmed by the NMR dissolution data (see Figure 4.16).

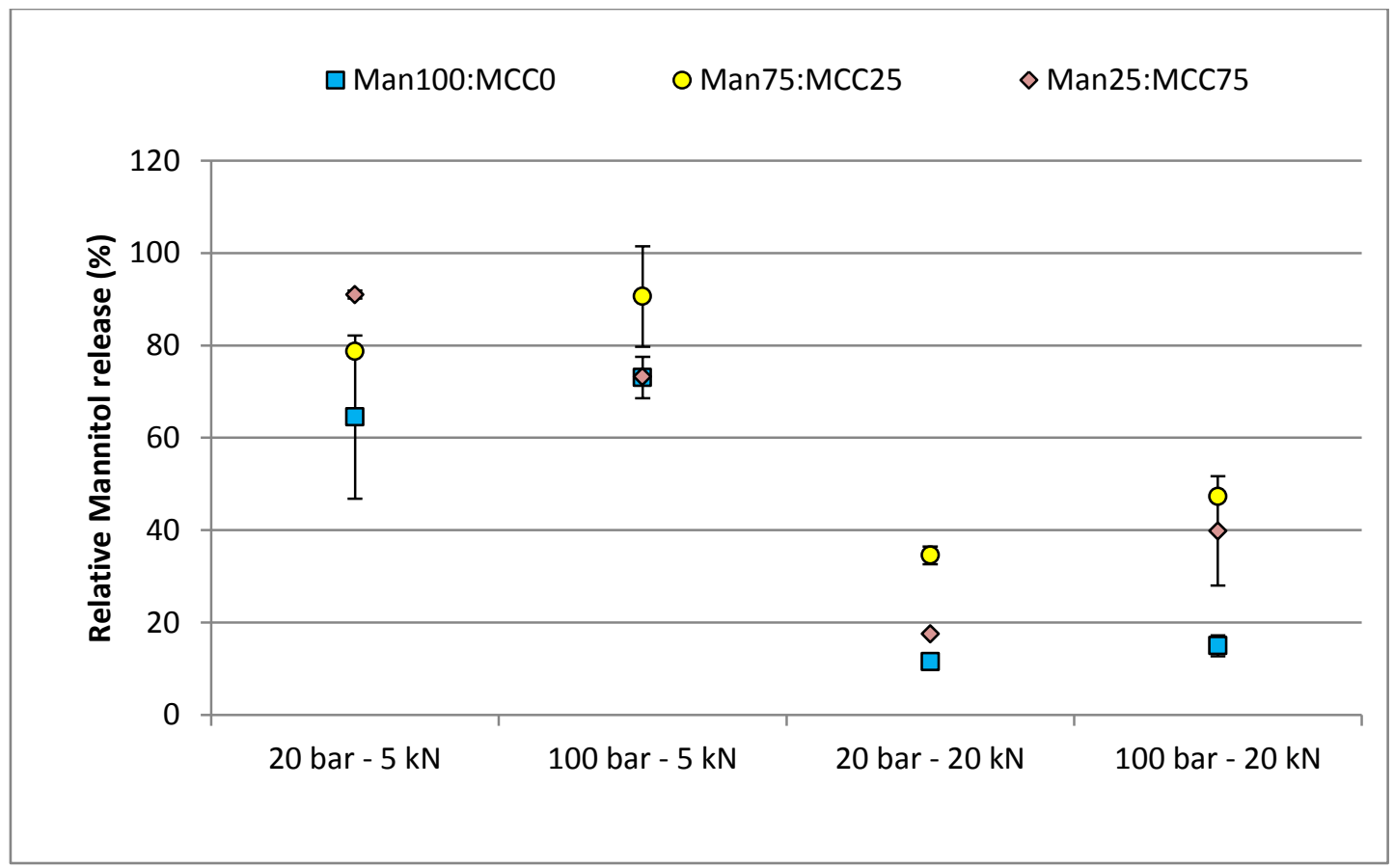

Figure 4.16 The relative mannitol release at $60 \mathrm{~s}$ for Man100:MCC0, Man75:MCC25 and Man25:MCC75 tablets produced using 20 and $100 \mathrm{RC}$ pressure and 5 and $20 \mathrm{kN}$ tableting force. 


\subsection{Conclusions}

In this chapter the effects of tableting force and RC pressure on tablet disintegration were examined using a modified USP apparatus which was connected to a particle imaging size measurement equipment. This was done to determine whether the method is capable of distinguishing the changes in formulation and processing parameters. This analysis method has the capabilities to determine the size of the released particles and the number of particles entrained with time. In conjunction with this, ${ }^{1} \mathrm{H}$ NMR was used to simultaneously measure the dissolution of mannitol.

By using the LIXELL it was possible to observe a difference in the cumulative particle release depending on the formulation used, as a higher percentage of MCC in the tablet formation was observed to release a higher number of particles. However, it was found that for tablets containing varying ratios of mannitol and MCC the LIXELL system was unable to precisely determine the effect of the stresses used during roller compaction and tableting on the number of particles released. This may be due to the mechanical stresses that the method subjects on the tablet due to it re-circulating the disintegration medium and due the agitation caused by the paddle stirrer.

Tablets containing MCC released smaller particles than Man100:MCC0 tablets. This is due to MCC being a disintegrant. The LIXELL particle imaging method presented high error when detecting the released particle sizes, probably due to the method being off-line. Therefore, the particles must travel and undergo various mechanical stress before detection occurs, meaning that the measurement is imprecise and prone to high error. 
${ }^{1} \mathrm{H}$ NMR was used to determine the dissolution rate of mannitol for the tablets which were analysed. Generally, the tablets produced using a higher tableting force presented a slower mannitol dissolution rate than when a low tableting force was used. It was also found that Man100:MCC0 tablets exhibited the slowest mannitol dissolution. This may be due to the volume of the released particles being higher, which could suggest that less mannitol is in solution.

There are numerous factors which influence the rate at which particles are released from a tablet and the rate at which mannitol dissolves into solution. This is dependent on the formulation and processing parameters used. These factors include the tablet porosity, the tablet tensile strength, the number of particles released and the size of the particles which can all influence the rate of release. An on-line method used to analyse disintegration would be more favourable as it would reduce the error which was observed during the LIXELL method. 


\section{Chapter 5. Flow Cell Imaging}

\subsection{Introduction}

Previously, in Chapter 4 an "on-line" particle imaging method was utilised for tablet disintegration analysis, however it was found to be unsuitable. This was due to the mechanical stress the tablet undergoes during the measurement from agitation by the paddle stirrer. Additionally, the particles which are released from the tablet must travel a significant distance before being detected by the particle analysis equipment. So some information is lost in transition and the particle may experience further breakage before being detected. Significant error was observed in the data obtained from this measurement, therefore a more on-line approach must be explored.

The aim of this Chapter is to gain a better understanding of tablet disintegration using an on-line particle imaging approach. A purpose-built Flow Cell was employed which is capable of on-line observation of tablet disintegration as it occurs, and which is able to visualise and measure the change in tablet area and rate of particle release (Mesnier et al., 2013). This additional information, which is obtained as disintegration is occurring, could facilitate a better understanding of tablet behaviour, and consequently led to more proficient tablet design. 


\subsection{Materials}

The details of the placebo binary mixtures of mannitol and MCC which will be analysed in this Chapter are outlined and characterised in Section 3.2.

\subsection{Methods}

\subsubsection{Granulation and Tableting}

The granulation and tableting parameters used to produce the tablets which were analysed in this Chapter are outlined in Sections 3.3.2 and 3.3.6, respectively. Table 5.1 shows the formulations and processing parameters used in this Chapter. Man0:MCC100 was not able to be analysed using the Flow Cell due to the tablet swelling extensively and not releasing particles with time, therefore a measurement could not be taken (see Appendix 19)

Table 5.1 The formulations analysed and their abbreviations. RC pressure and tableting force is also specified.

\begin{tabular}{|l|cccc|}
\hline Abbreviations & $\begin{array}{c}\text { Mannitol } \\
(\%)\end{array}$ & MCC (\%) & $\begin{array}{c}\text { Roller compaction } \\
\text { pressure (bar) }\end{array}$ & $\begin{array}{c}\text { Tableting } \\
\text { force (kN) }\end{array}$ \\
\hline Man100:MCC0 & 100 & 0 & 20,100 & 5,20 \\
\hline Man75:MCC25 & 75 & 25 & 20,100 & 5,20 \\
\hline Man50:MCC50 & 25 & 75 & 20,100 & 5,20 \\
\hline Man25:MCC75 & 25 & 75 & 20,100 & 5,20 \\
\hline
\end{tabular}

\subsubsection{Flow Cell disintegration analysis}

A real-time imaging method was used to monitor tablet disintegration of various tablets. A purpose-built Flow Cell (shown in Figure 5.1) was used for tablet disintegration analysis. The Flow Cell has an inner diameter of $5 \mathrm{~cm}$ and a length of 
$15 \mathrm{~cm}$. The Flow Cell contains a mesh platform for the placement of the tablet and a viewing window to enable direct imaging of the tablet and the particles which are released from the tablet. Tablets were held in place by using Scotch $8 \mathrm{~mm}$ circular adhesive dots. A mixture of $1 \mathrm{~mm}$ and $2 \mathrm{~mm}$ glass beads were placed in the lower compartment to ensure a more uniform water flow through the cross-sectional area of the cell where the tablet is placed. An RS Centrifugal Pump (Northants, UK), capable of pumping at $2.8 \mathrm{l} / \mathrm{min}$, was used to pump water using $6 \mathrm{~mm}$ tubing from a Clifton NE1-8 unstirred water bath (Weston-Super-Mare, UK) which maintained the water temperature at $25^{\circ} \mathrm{C}$. The water was introduced into the Flow Cell through the lower inlet into the cell and exited through the upper outlet into a basin. This ensured that released particles from the tablet were carried away in the same direction as the water flow, and could be imaged without being counted twice. A Canon (Tokyo, Japan) EOS 1D Mark IV 16 MP camera, fitted with a Canon (Tokyo, Japan) MP-E $65 \mathrm{~mm}$ macro lens, was used to obtain images at a rate of $1 \mathrm{fps}$. Uniform lighting within the Flow Cell was achieved using an external Zeiss (Oberkochen, Germany) 1800 LCD light source to ensure that the objects could be viewed clearly. 


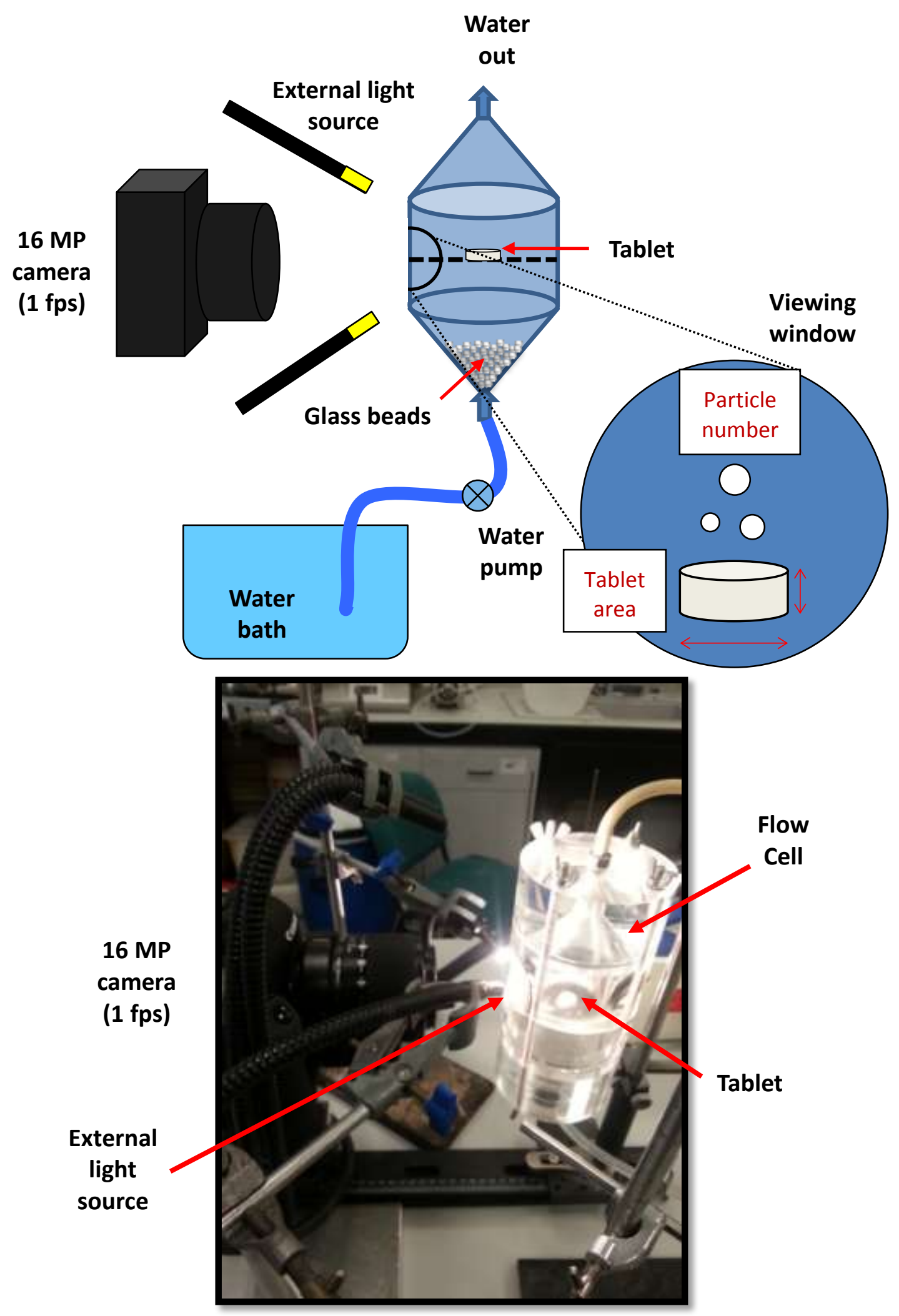

Figure 5.1 Schematic and image of the Flow Cell set-up. 
A MATLAB (Mathworks Inc., USA) program (see Appendix 18) was utilised to process and analyse the images obtained during measurement. Firstly, during image processing the images are thresholded to produce a binary image, with objects (tablet and particles) having a value of 1 and background pixels a value of 0 . Objects within the binary images are identified by detecting collections of pixels with a value of 1 ; with the largest collection being identified as the tablet and the remaining being identified as released particles. The $2 \mathrm{D}$ tablet area is calculated by counting the number of pixels within this area and relating it to the image scale calibration. The number of particles released with time is obtained by totalling the number of pixel clusters.

With this method 3 tablets were tested per processing condition, and an average of the 3 measurements was analysed. One data point per second was obtained during the measurements; to smooth the data a Savitzky Golay Filter (Savitzky and Golay, 1964) was applied. A Savitzky Golay Filter applies a moving polynomial fit, much like a moving average, to smooth noisy data. In this case a subsample distance of 10 and a polynomial order of 2 was used to smooth the Flow Cell data. The camera placement (distance from the Flow Cell), the tablet placement and the camera focus were established so that the full tablet was clearly visible and focused within the camera viewing window during the entirety of the measurement, taking in to account tablet swelling. The water flow rate which was used during analysis $(2.8 \mathrm{l} / \mathrm{min})$ was selected due to the material MCC requiring a high flow rate to effectively carry particles out of the Flow Cell once they become saturated with water. With lower flow rates saturated MCC particles were found to sink back to the mesh platform. This caused some inconsistencies in the data obtained whereby the particles are observed as being part of the tablet structure. 
It was attempted to connect a conductivity meter to the outgoing water from the Flow Cell during a disintegration measurement to determine the dissolution of mannitol with time. It was unsuccessful due to the high volume of water used during each Flow Cell measurement. The concentration of conductive material was unable to build up to a detectable level, therefore conductivity measurements failed.

\section{Fluid Reynolds number}

The Fluid Reynolds number (Re) was calculated using Equation 5.1 (Rott, 1990).

$$
\operatorname{Re}=\frac{\rho u D}{\mu}
$$

where $\rho$ is the density of the fluid $\left(1000 \mathrm{~kg} / \mathrm{m}^{3}\right)$, $\mathrm{u}$ is the velocity of the fluid at the point where the tablet is placed in the Flow Cell $(0.023 \mathrm{~m} / \mathrm{s}), \mathrm{D}$ is the diameter of the Flow Cell $(0.05 \mathrm{~m})$ and $\mu$ is the viscosity of the fluid $\left(8.9 \times 10^{-4}\right)$.

The Reynolds number was calculated to be $\operatorname{Re}=1292$, which ensures laminar flow within the Flow Cell.

\subsection{Results and Discussion}

\subsubsection{Imaging}

The tablet disintegration was monitored using a purpose-built Flow Cell which allows for the imaging of tablet disintegration in real-time. Figure 5.2 and Figure 5.3 show the images that were captured during the analysis taken at varying intervals. The images obtained showed that on contact with water tablets tended to swell in size then erode, i.e. decrease in size due to particle release. The extent to 
which the swelling and erosion behaviour occurs during the tablet disintegration process is dependent on the formulation and the processing parameter used.

Figure 5.2 shows example images for tablets compacted using a tableting force of $5 \mathrm{kN}$. Tablets containing a higher percentage of mannitol (e.g. Figure 5.2a and Figure 5.2b) expand slightly and then disintegrate relatively quickly. This is due to the mannitol being a soluble material meaning that tablet material is lost through particle release as well as through dissolution.

With the addition of MCC into the tablet formulation the tablet swelling is more pronounced at the $1 \mathrm{~s}$ time point; this is characteristic of MCC which tends to expand on contact with water. The decrease in the tablet area for tablets containing higher percentages of MCC is also slower than tableting containing higher percentages of mannitol.

Additionally, due to the insoluble nature of MCC, it was found that MCC becomes saturated with water and dwells on the Flow Cell platform rather than being transported and removed with the flow of the water. This means that material is remaining in the field of view of the measurement even after tablet disintegration may have reached completion.

When looking at the effect of RC pressure, there is no clear visual difference in the swelling or erosion behaviour for tablets produced using a low tableting force depending on the RC pressure. 


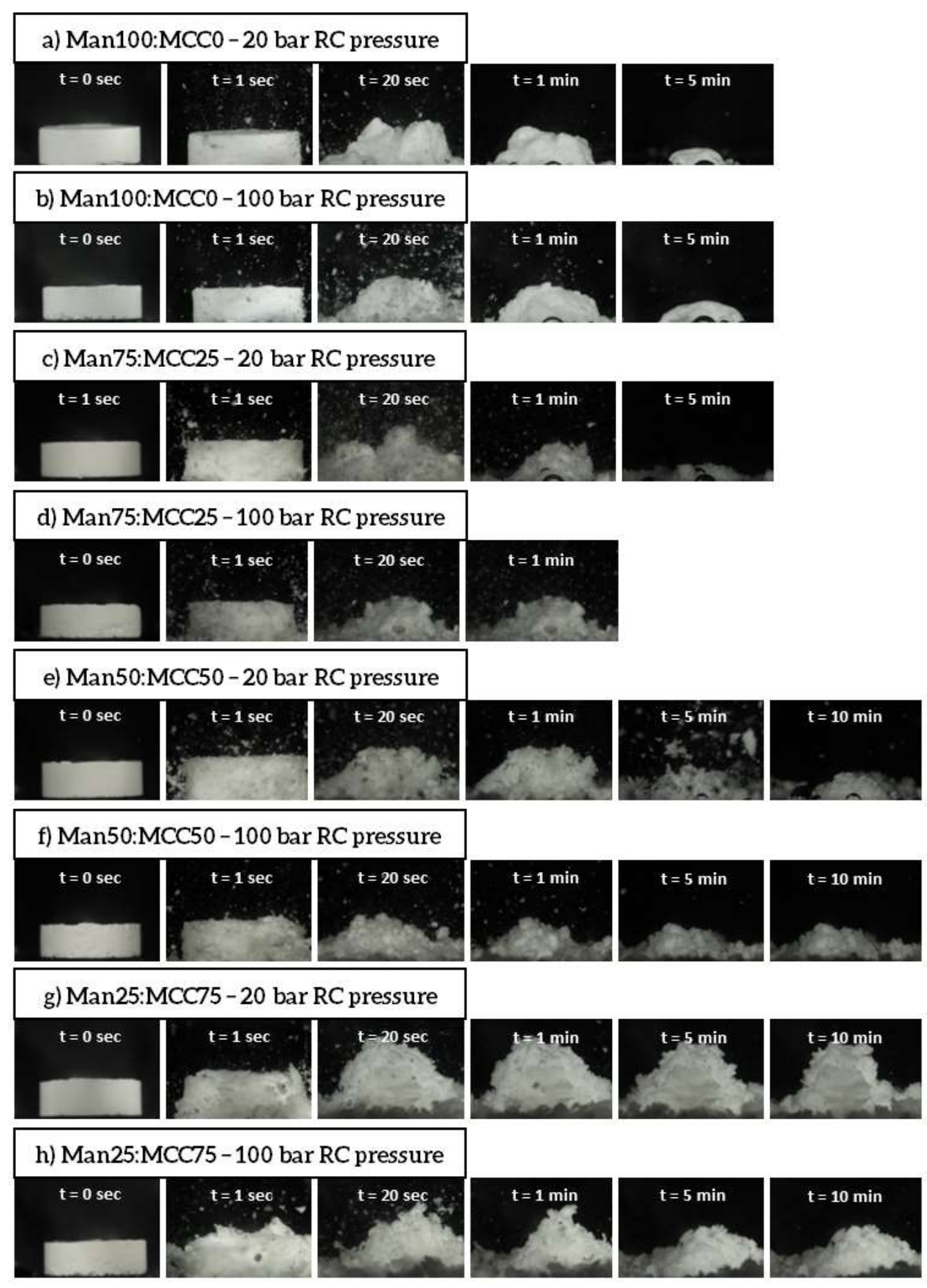

Figure 5.2 Images taken during Flow Cell analysis of tablet disintegration for tablets produced using $5 \mathrm{kN}$ tableting force for a) Man100:MCC0, RC pressure $20 \mathrm{bar}$; b) Man100:MCC0, RC pressure 100 bar; c) Man75:MCC25, RC pressure 20 bar; d) Man75:MCC25, RC pressure 100 bar; e) Man50:MCC50, RC pressure 20 bar; f) Man50:MCC50, RC pressure 100 bar; g) Man25:MCC75, RC pressure 20 bar; h) Man25:MCC75, RC pressure 100 bar. 
Example images for tablets compressed using a tableting force of $20 \mathrm{kN}$ are shown in Figure 5.3. Generally with a higher tableting force the erosion time was observed to be prolonged. In other words, the tablet area did not decrease after swelling occurred in some cases. It was also observed that the disintegration of the tablet was much gradual for tablets compacted using $20 \mathrm{kN}$; the time taken to reach the maximum tablet area after swelling was much longer than the comparable tablets produced using $5 \mathrm{kN}$.

Notably, tablets produced using 20 bar RC pressure and high tableting force were found to disintegrate via a different mechanism to what was seen for tablets produced using other parameters. Rather than eroding with time while releasing particles, the tablet swelled and separated in the centre region and layers of compacted material were visible. For Man100:MCC0 tablets a more curved semicircle shape was observed (Figure 5.3a). Following the separation of the tablet into 2 sections the tablet then dissolves rather than eroding into small particles. This suggests that shear planes and/or density distributions exist within the tablet which manifests during tableting. 


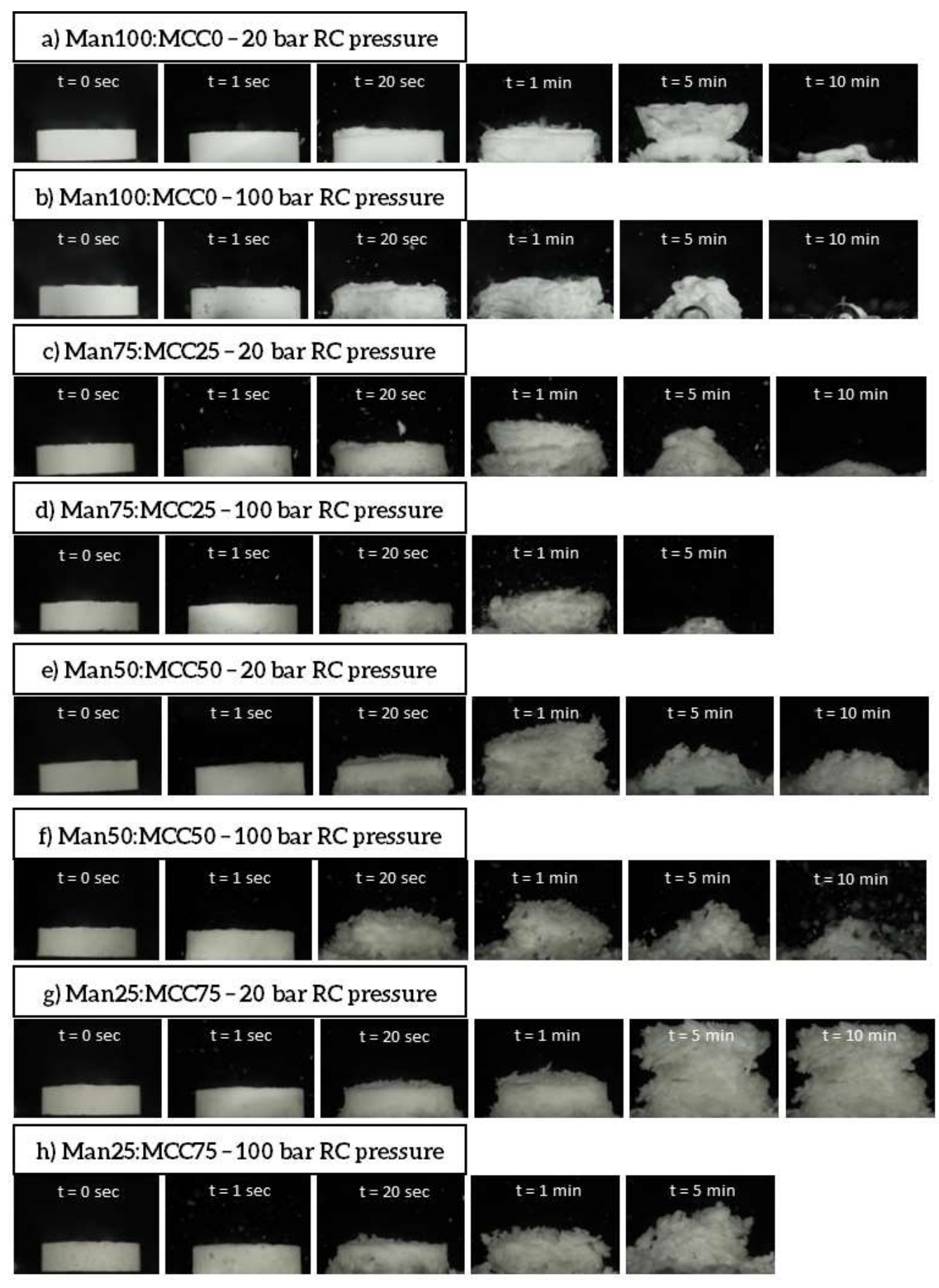

Figure 5.3 Images taken during Flow Cell analysis of tablet disintegration for tablets produced using $20 \mathrm{kN}$ tableting force for a) Man100:MCC0, RC pressure $20 \mathrm{bar}$; b) Man100:MCC0, RC pressure 100 bar; c) Man75:MCC25, RC pressure 20 bar; d) Man75:MCC25, RC pressure 100 bar; e) Man50:MCC50, RC pressure 20 bar; f) Man50:MCC50, RC pressure 100 bar; g) Man25:MCC75, RC pressure 20 bar; h) Man25:MCC75, RC pressure 100 bar. 
It can also be observed that tablets produced using granules compacted at 20 bar RC pressure swelled more significantly than when 100 bar RC pressure was used. From the compactibility graphs (Section 3.4.3) it was determined that higher stress applied during tableting produces stronger tablets. And from the Flow Cell images it was seen that the tablets that have had more stress applied tend to swell in size more in size. This is due to the application of higher stress leading to stronger particle-particle bonding occurring. So when water intake is much slower and it causes significant swelling to occur before the bonding is weakened. When the bonding becomes too weakened the bonding begins to break and particles are released.

A common trend which was observed is that the top corners of the tablet disintegrated before the centre of the tablet in most cases. This tablet erosion behaviour could be due to the water velocity variations at different points on the tablet. The edges of the tablet are subjected to a higher velocity of water therefore, the edge erode more rapidly than the centre of the tablet (see Figure 5.4). This leads to the tablet area shape observed in the images obtained through the Flow Cell. 


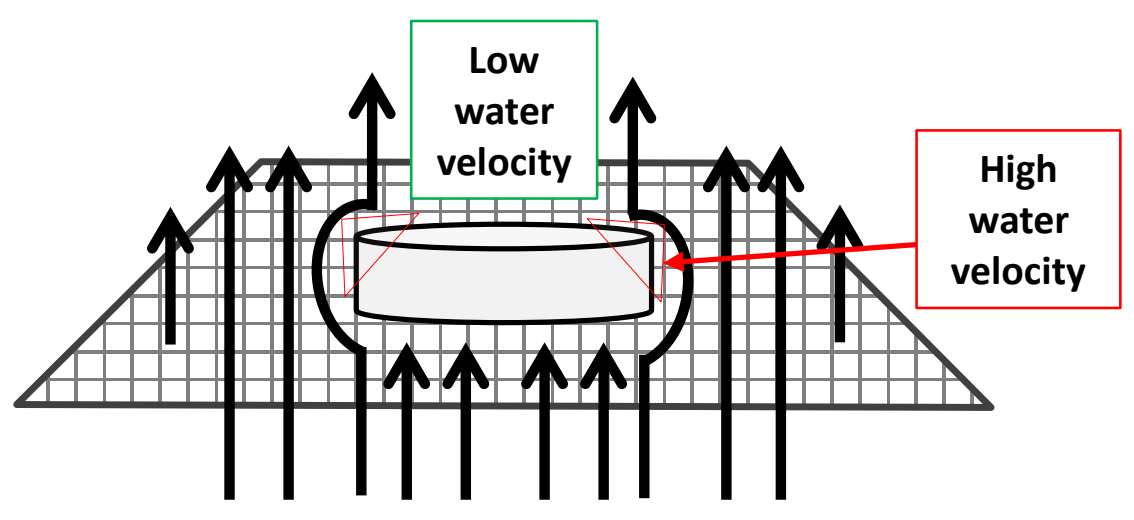

Figure 5.4 Schematic of the water flow and velocity acting on the tablet inside the Flow Cell.

\subsubsection{Tablet area and particle release}

The change in tablet area with time (see Figure 5.2 and Figure 5.3) is a visual indication of tablet disintegration. The tablet disintegration as observed in the imaging method shows that the tablet swells in size, before undergoing a secondary stage where the tablet begins to erode and decrease in size due to particle release and dissolution. From the images it was possible to detect these changes in tablet area and the particle release rate in real-time using a MATLAB program (see Appendix 18). The areas were calculated by counting the pixels in the images, then they were normalised by dividing the changing tablets areas by the initial tablet area.

Figure 5.5 shows the evolution of the normalised tablet area tablets with time. Tablets which were compacted using a low tableting force of $5 \mathrm{kN}$ for varying ratios of mannitol and MCC granulated at 20 and 100 bar RC pressure are shown. As previously observed in the images which were presented in Figure 5.2, the tablet first swells to a maximum area. Then the tablet begins to erode due to particle 
release and dissolution. For tablets produced using $5 \mathrm{kN}$ tableting force, the effect of variation of the RC pressure was not able to be observed. Tablets containing both 20 and 100 bar granules swelled and disintegrated in a similar manner, despite the fact the tablet porosities varied significantly (see Figure 3.11).

Figure 5.5a shows that disintegration is rapid for Man100:MCC0 tablets. Swelling of the tablets reached a maximum at approximately $20 \mathrm{sec}$. Erosion after swelling also occurs relatively rapidly, with tablet area reaching near 0 . When the percentage of MCC within the formulation is increased the tablet swelling was comparable to Man100:MCC0 tablets.

Generally the addition of MCC was found to prolong the disintegration time. In other words, the tablet does not erode meaning that the tablet area does not decrease as readily. With the addition of MCC into the formulation the disintegration stage after swelling is less pronounced. For tablets containing any amount of MCC the tablet area does not decrease as quickly as tablets containing only mannitol. Tablets which contain $50 \%$ or more MCC do not exhibit a decrease in tablet area below the original area; this is due to the MCC becoming saturated with water and resisting being carried away with the water flow.

It should be noted that for Man0:MCC100 tablets was it was not possible to carry out the Flow Cell analysis as the tablet swells significantly but does not change in area following the swelling stage (see Appendix 19). Particles are also not released and therefore Man0:MCC100 tablets were deemed to be not viable for measurement using the Flow Cell. 


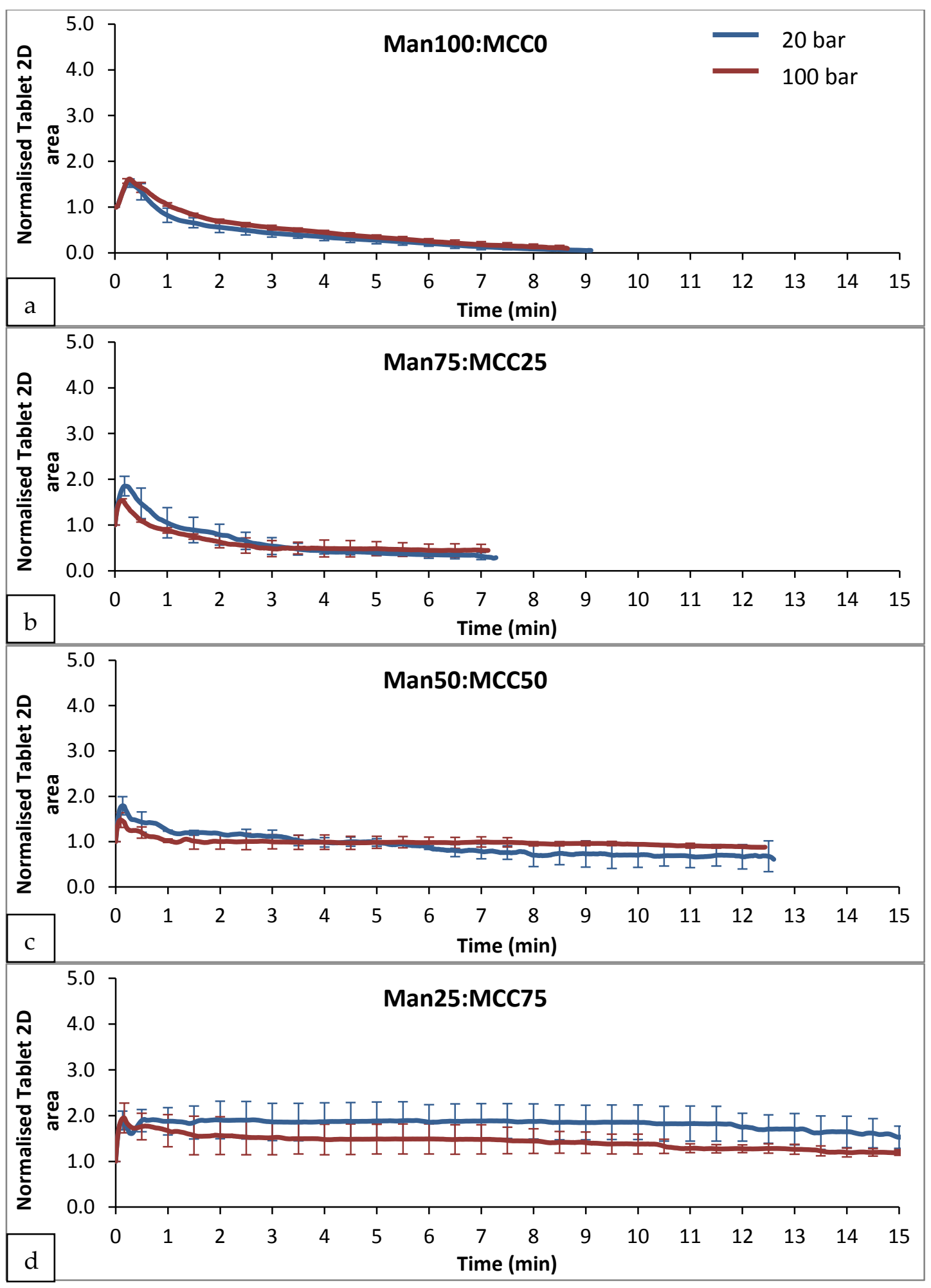

Figure 5.5 The change in the normalised tablet area with time for tablets with varying ratios of mannitol and MCC granules which were produced using RC pressure of 20 and 100 bar and a tableting force of $5 \mathrm{kN}$. 
Figure 5.6a shows the cumulative number of released particles with time for tablets produced using 20 and 100 bar RC pressure granules which were compacted at $5 \mathrm{kN}$ tableting force. Man100:MCC0 tablets (Figure 5.6a) released particles in a similar manner independent of the RC pressure used; initially there is a period of rapid particle release, followed by a period of slower particle release. The effect of RC pressure is not apparent for Man100:MCC0 tablets, which was also the case for the change in tablet area with time.

Man75:MCC25 tablets produced particles at a similar rate to Man100:MCC25 tablets. A slight difference was observed in the rate of release of particles depending on the RC pressure used. 20 bar granules appeared to release particles at a faster rate; however the error is high in this data to confirm this with certainty.

Tablets containing a high percentage of MCC (Figure 5.6c and Figure 5.6d) which were produced using 20 bar RC pressure granules also appeared to release particles at a faster rate than the equivalent 100 bar granule tablets. This difference in release rate is more pronounced for tablets containing MCC as it is an insoluble material; therefore observation of a higher number of overall particles is more likely. However, it must be taken into consideration that the error in some cases is significant, therefore there is some uncertainty in the data.

From the X-ray measurements (see Section 3.4.4) it was observed that the tableting stress being applied in this case sufficient to crush a significant amount of the 20 bar granules, causing a larger number of weakly bonded smaller particles to be present within the tablet. The Flow Cell data supports this as the tablets produced using 20 bar granules appear to release a greater total number of particles. 


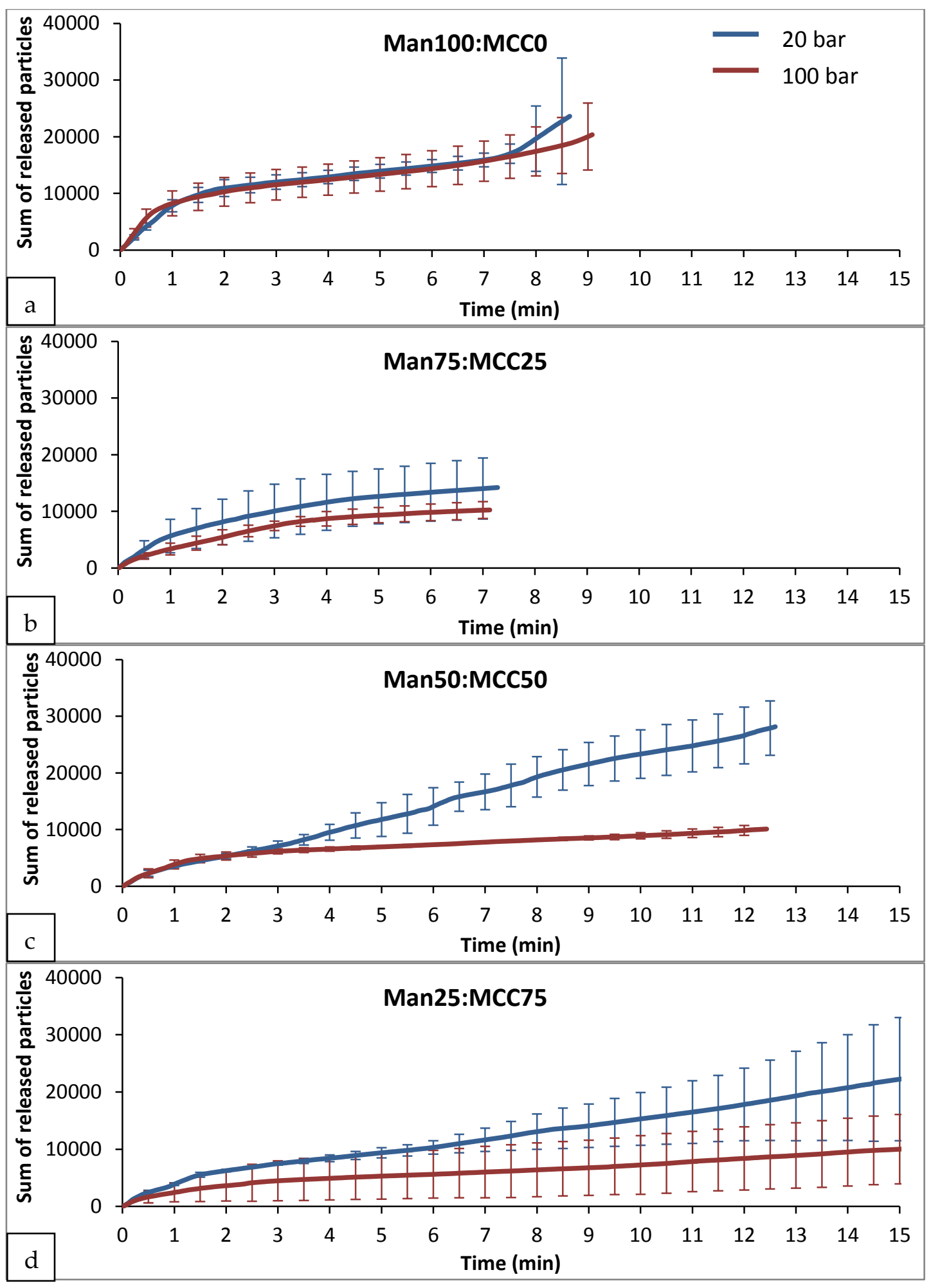

Figure 5.6 The total number of released particles with time for tablets with varying ratios of mannitol and MCC granules which were produced using RC pressure of 20 and 100 bar and a tableting force of $5 \mathrm{kN}$. 
The change in tablet area with time for tablets compacted using a higher tableting force of $20 \mathrm{kN}$ are shown in Figure 5.7. At $20 \mathrm{kN}$ tableting force, the Man100:MCC0 tablets disintegration was highly dependent on the RC pressure used (see Figure 5.7). With 100 bar RC pressure, the tablets did not swell as highly as the 20 bar tablets. The maximum tablet area was also reached at a quicker time (2.5 min). Whereas a low RC pressure of 20 bar, the tablets swelled to around 2 times the maximum tablet area, and reached this area at the slowest time of $5.1 \mathrm{~min}$. The overall disintegration time was prolonged due to the higher tableting force used.

In all cases, tablets containing granules produced using $20 \mathrm{kN}$ tableting for and 20 bar RC pressure swelled to a higher tablet area than 100 bar. Additionally, the overall time taken for the tablet to erode, i.e. decrease in size, to an equivalent tablet area following erosion was longer for 20 bar tablets.

With increasing MCC percentage in the formulation a significantly higher tablet swelling was observed. Furthermore, the addition of MCC in the formulation led to a prolonged erosion time, which is due to the uptake of water into the MCC causing it to become saturated and unable to fully and completely disintegrate (see Figure 5.7c and Figure 5.7d). This caused the tablet particles to remain on the platform rather than eroding away and decreasing in area. This was especially the case for Man25:MCC75 tablets for which no change in tablet area was observed between $6-15$ mins. 


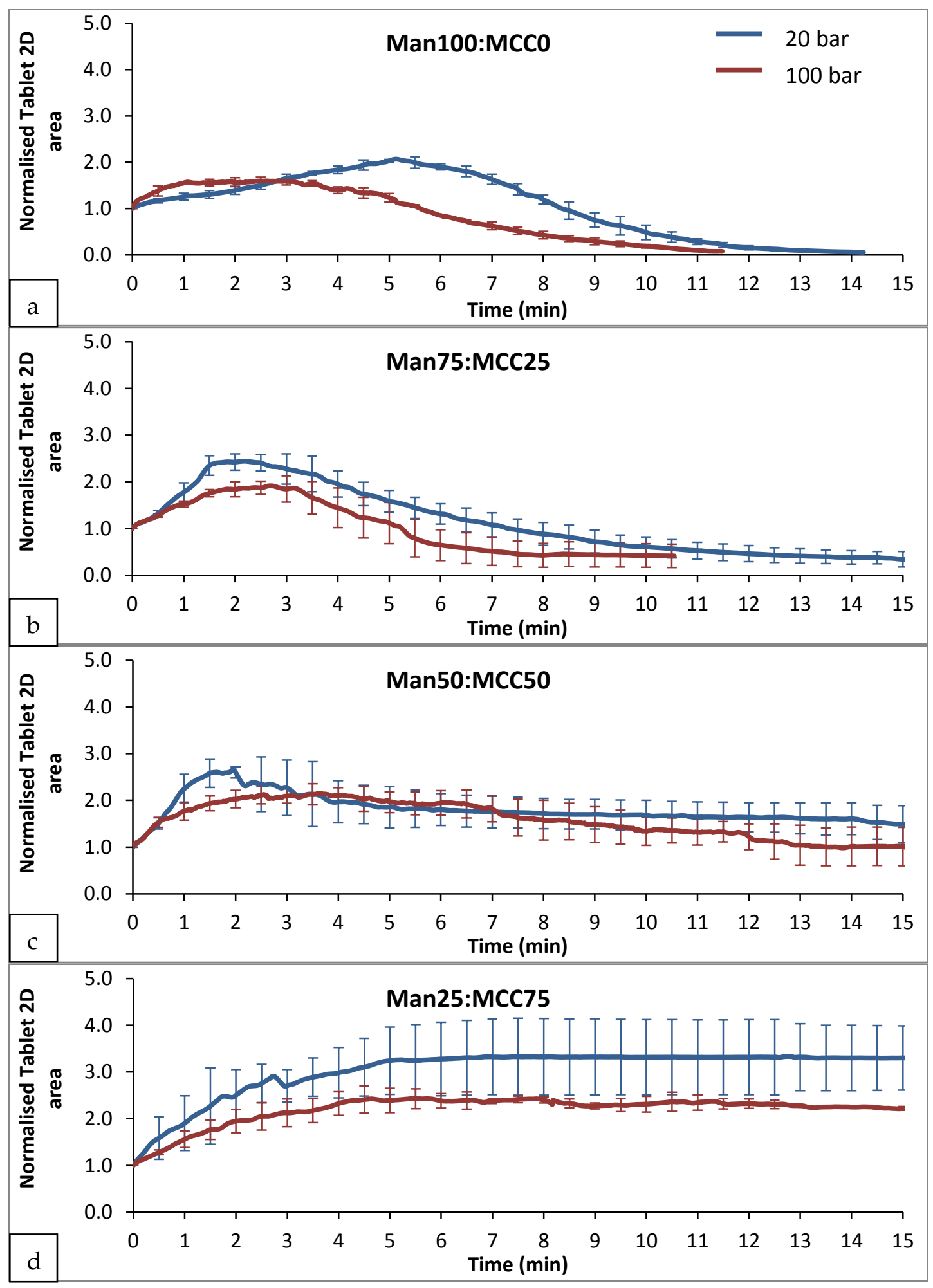

Figure 5.7 The change in the normalised tablet area with time for tablets with varying ratios of mannitol and MCC, produced using a tableting force of $20 \mathrm{kN}$. 
The effect of tableting force on tablet disintegration is quite clear; a higher tableting force led to higher tablet swelling and a prolonged erosion time. This is due to the material becoming more compacted therefore water intake is slower. And due to the higher tableting stress applied the bonding within the tablet is much stronger and consequently disintegration is delayed.

When a higher force of $20 \mathrm{kN}$ was applied to produce the tablets, the effect of $\mathrm{RC}$ pressure was less apparent in terms of the particle release (Figure 5.8). Generally tablets containing granules produced using 20 and 100 bar RC pressure both released particles at a similar rate (Figure 5.8a - Figure 5.8c). With the exception of Man25:MCC75 tablets (Figure 5.8d) for which 20 bar granules led to a faster particle release rate. However, the error in all cases is high and a conclusive difference in the particle release depending on the RC pressure used cannot be elucidated from the data.

In terms of the release rate, all tablet formulations released particles at a comparable rate. From X-ray analysis of the tablet internal structure it was found that when a higher tableting force is used, the granular material largely becomes crushed. Tablets containing 100 bar granules showed that the granular structure is partially visible (see Figure 3.14), however the granular material is strongly and densely bonded. The granular structure no longer has any influence. This supports the particle release data as we see similar particle release numbers for each of the formulations (see Figure 5.8). 


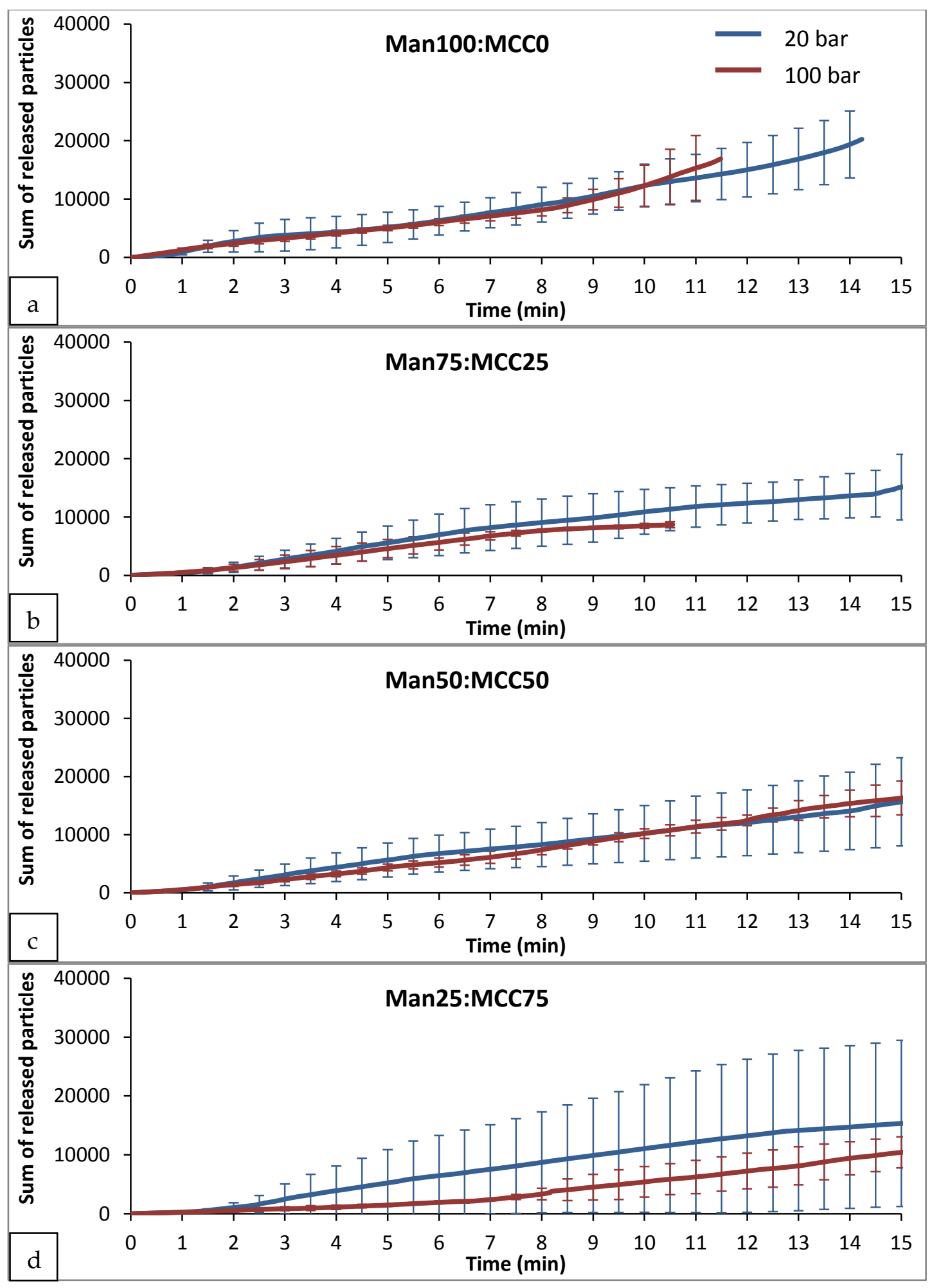

Figure 5.8 The total number of released particles with time for tablets with varying ratios of mannitol and MCC, produced using a tableting force of $20 \mathrm{kN}$. 


\subsection{Conclusions}

The Flow Cell analysis of tablet disintegration found that at low tableting force the Man100:MCC0 tablets disintegrated in a similar manner regardless of the RC pressure used. For tablets containing MCC, a lower RC pressure led to slightly more swelling during disintegration. At high tableting force the disintegration behaviour for all tablets tested varied depending on the RC pressure used. In all cases a lower RC pressure led to more tablet swelling and a slower disintegration time. However, the error in the data was relatively high so the trends observed cannot be confirmed.

From the Flow Cell images it was observed that the addition of MCC in the tablet formulation leads to higher tablet swelling and a longer disintegration time. Similarly, when a higher tableting force was applied to the tablet, the time taken for the tablet to swell to the maximum area and then to erode was delayed due to stronger bonding within the tablet, therefore water uptake was slower.

In terms of the particle release, the data suggested that at low tableting force the particle release rate was in some cases dependent on the RC pressure used. If a low RC pressure was used during granulation, a faster particle release rate was observed from the consequent tablets. From the X-ray imaging it was found that the weaker granular material is being crushed into a smaller granules and primary powder during tableting. This, in addition to the weakness of the tablet, may lead to

a faster release rate of a higher number of particles for these tablets containing weaker granular material.

At higher tableting force it was found that the particle release is similar for both high and low RC pressures. This is due to the stress being high enough to 
crush the granular material and bond it strongly, in most cases, during tableting. Therefore, the particle release rate is slow and the number is almost identical and independent of the RC pressure used previously.

Overall, it was determined that many factors affect the tablet swelling and erosion of tablets. The differences observed due to the material properties were clear; MCC swelled and prolonged the erosion time, whereas mannitol dissolved relatively quickly. The stress applied during tableting and RC also played a part. The porosity and bonding within the tablet (i.e. the tensile strength) affected the disintegration time how the tablet swelled. 


\section{Chapter 6. USP vs Flow Cell Imaging}

\subsection{Introduction}

In the previous Chapters it was determined that the effect of different filler excipients on the tablet disintegration was apparent and significant. The aim of this Chapter is to compare the Flow Cell, a particle imaging approach, and the conventional USP disintegration analysis technique to monitor tablet disintegration of a placebo formulation. A placebo formulation contains a mixture of excipients, each with a function, but no active pharmaceutical ingredient (API).

A purpose-built Flow Cell, which was previously explored in Chapter 5, was employed which is capable of on-line observation of tablet disintegration. This technique can provide information about the changing tablet dimensions and the particles released with time. This additional information can improve the understanding of how different materials and process parameters affect tablet disintegration. Standard USP analysis was also carried out which is able to determine the disintegration time (see Section 1.3). It will be evaluated whether the USP method can differentiate the disintegration behaviour of tablets produced using different formulations and processing parameters.

In this Chapter a placebo pharmaceutical formulation was analysed to investigate which disintegration analysis method is more informative when a tablet contains many components, as it would in a pharmaceutical solid unit dosage. It 
will also be determined which, if any, of the analysis techniques is able to detect variation in the formulation, $\mathrm{RC}$ stress and tableting stress used.

\subsection{Materials}

In this Chapter placebo pharmaceutical formulations will be examined. The excipient materials were used as received, unless otherwise stated.

\subsubsection{Fillers}

Microcrystalline cellulose Avicel PH101 from Sigma Aldrich (St. Louis, USA) and Mannitol Pearlitol 200SD from Roquette (Lestrem, France) were used as fillers. Further information is provided in Section 3.2.1 and Section 3.2.2.

\subsubsection{Binder}

Povidone Kollindon 30 from BASF (Geismar, Germany) was used as a binder. Povidone is a fine, white, hygroscopic powder. Commonly used as a binder with both wet and dry granulation to produce solid dosage forms (Rowe et al., 2009).

\subsubsection{Disintegrant}

Sodium starch glycolate from Roquette (Lestrem, France) was used as a disintegrant. It is structurally described as a crosslinked carboxymethyl ether of starch which disintegrates due to rapid uptake of water and consequent swelling (Rowe et al., 2009). It is commonly used in the pharmaceutical industry in various oral dosage systems.

\subsubsection{Lubricant}

Magnesium stearate from Mallinkrodt (St. Louis, USA) was used as a lubricant. 


\subsubsection{Placebo formulation}

Table 6.1 shows the two placebo formulation analysed in this Chapter, containing varying ratios of fillers MCC and mannitol, sodium starch glycolate as a rapid disintegrant, povidone as a binder and magnesium stearate as a lubricant. The formulations contained either an excess of mannitol or an excess of MCC, hence the abbreviated names.

Table 6.1 The abbreviations for the placebo formulations.

\begin{tabular}{|cccc|}
\hline Materials & \multicolumn{2}{c|}{ Abbreviations } & $\begin{array}{c}\text { Solubility } \\
\text { in water }\end{array}$ \\
\hline Mannitol (\%) & ExMCC & ExMan & $\checkmark$ \\
\hline MCC (\%) & 15 & 75 & $\times$ \\
\hline Sodium starch glycolate (\%) & 35 & 15 & $\times$ \\
\hline Povidone (\%) & 5 & 3 & $\times$ \\
\hline Magnesium stearate* (\%) & 2 & 5 & 2 \\
\hline
\end{tabular}

*The full amount of lubricant was added prior to granulation

\subsection{Methods}

\subsubsection{Granulation and tableting}

The granulation and tableting parameters used to produce the tablets which were analysed in this Chapter are outlined in Sections 3.3.2 and 3.3.6, respectively. The three ExMan formulations are outlined in Table 6.2; this is due to the variation in the processing parameters. RC pressures of 20 or 50 bar and tableting forces of 15 or $25 \mathrm{kN}$ were used to produced tablets. 
Table 6.2 The RC pressures and tableting forces used to produce tablets.

\begin{tabular}{|cccccc|}
\hline & ExMCC-A & ExMan-A & ExMan-B & ExMan-C \\
\hline RC pressure (bar) & 20 & 20 & 20 & 50 \\
\hline Tableting force (kN) & 25 & 25 & 15 & 15 \\
\hline
\end{tabular}

In this study a comparison of the variation in formulation (ExMCC-A vs ExMan-A), variation of the RC pressure (ExMan-B vs ExMan-C) and a variation in the tableting force (ExMan-A vs ExMan-B) will be examined.

\subsubsection{Granule size distribution}

The granule size distribution was determined by using the method specified in Section 3.3.5.

\subsubsection{Tablet tensile strength and porosity}

Tablet tensile strength was calculated by using the method outlined in Section 3.3.7. The tablet porosity $(\varepsilon т)$ was calculated using Equation 6.1 and Equation 6.2 (Gad, 2008). The true densities of the powders were obtained from the manufacturer. The tablet dimensions were measured using a precision slider calliper. Tablets were weighed using a Mettler Toledo AE 160 (Greifensee, Switzerland). 10 tablets were tested for each batch where either the formulation or processing parameter was varied. The average of the 10 values was calculated.

$$
\text { Apparent density }=\frac{\text { Tablet weight }}{\text { Tablet volume }} \quad \text { Equation } 6.1
$$$$
\varepsilon_{\mathrm{T}}=\left(1-\frac{\text { Apparent density }}{\text { True density }}\right) \times 100
$$ 


\subsubsection{USP disintegration analysis}

The disintegration time of the tablets was measured using a Copley ZT74 6 tablet tester (Nottingham, UK). A mesh size of $2 \mathrm{~mm}$ square was used and $800 \mathrm{ml}$ water heated to $37^{\circ} \mathrm{C}$ was used as the disintegration medium. At least 8 tablets were tested for each condition. The mean disintegration time of all of the tested tablets, and the time taken for the last (slowest) tablet to disintegrate were both recorded.

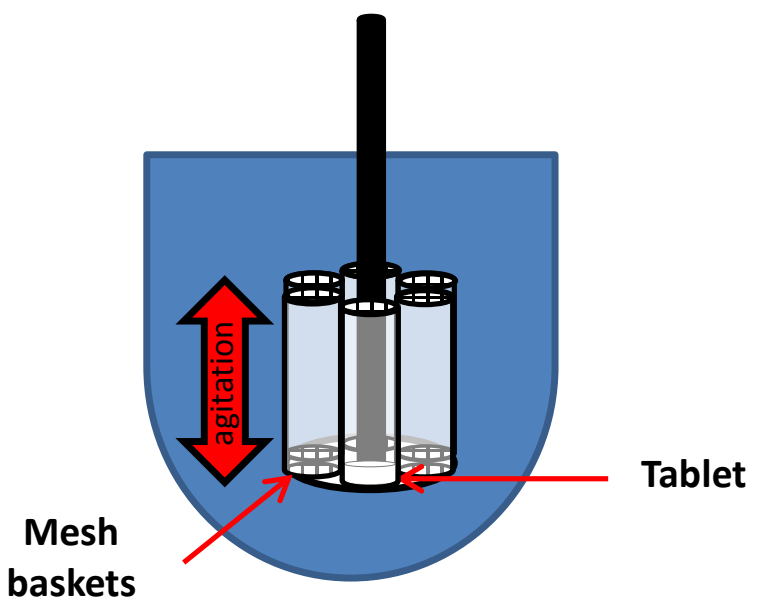

Figure 6.1 USP Basket-Rack assembly.

\subsubsection{Flow Cell disintegration analysis}

The Flow Cell apparatus used to monitor tablet disintegration is outlined in Section 5.3.2. The MATLAB code used to analyse the obtained images is outlined in Appendix 18. At least 5 tablets were tested per condition. In addition to the change ion tablet area and the number of particles, the particle size was also calculated by using the MATLAB code in Appendix 20. 


\subsection{Results and Discussion}

\subsubsection{Granule size distribution}

Figure 6.2 shows the $\mathrm{D}_{10}, \mathrm{D}_{50}$ and $\mathrm{D}_{90}$ granule size values for the excess MCC formulation and compressed at 20 bar roller compaction pressure (ExMCC-A), and the granules produced using the excess mannitol formulation which was compressed using 20 and 50 bar roller compaction pressure (ExMan-A and ExManC, respectively). It was found that all of the granules exhibited similar $\mathrm{D}_{10}$ and $\mathrm{D}_{90}$ values, however the $\mathrm{D}_{50}$ did vary. Firstly, when comparing the effect of formulation, ExMan-A granules had a higher D50 value than ExMCC-A granules.

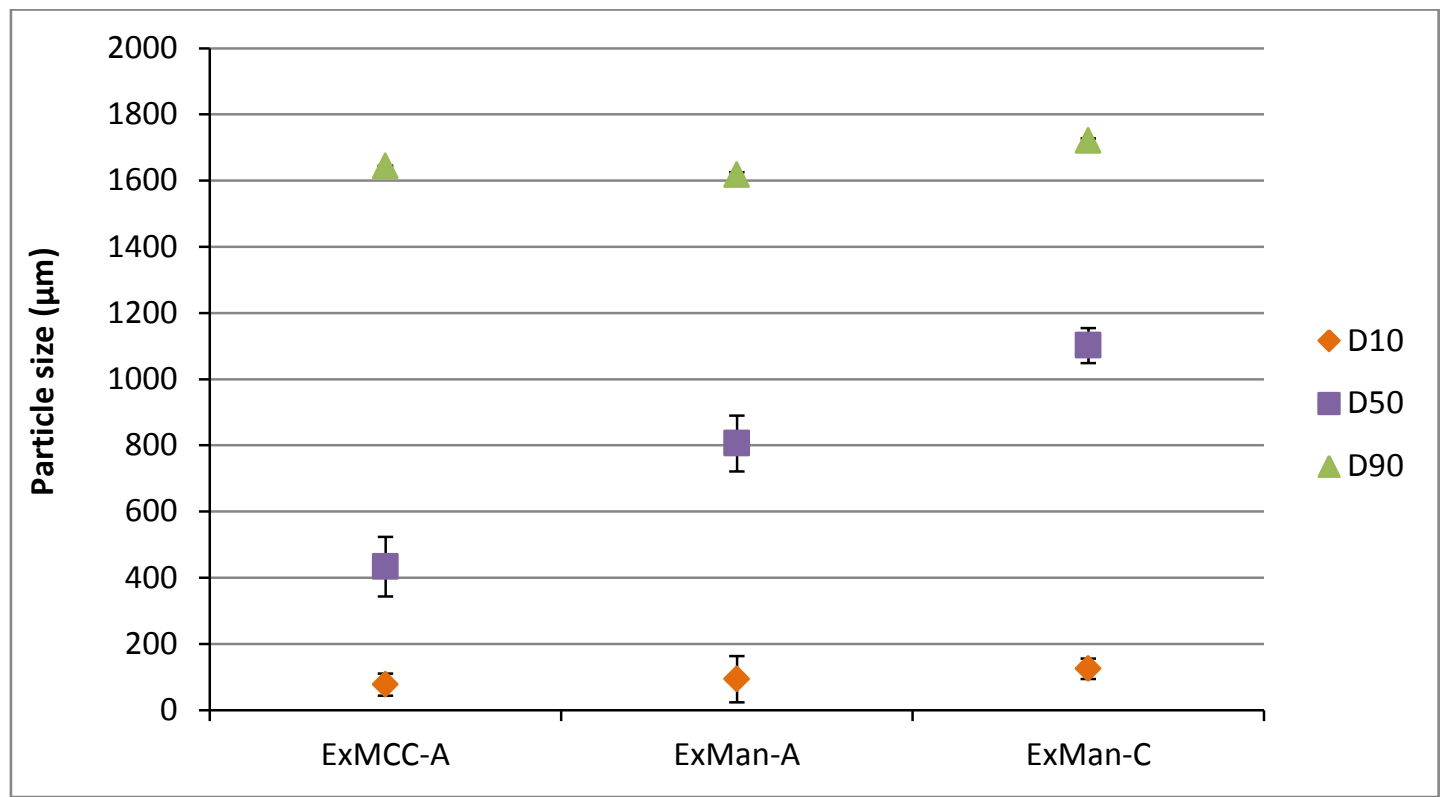

Figure 6.2 Volume-weighted granule $\mathrm{D}_{10}, \mathrm{D}_{50}$ and $\mathrm{D}_{90}$ data for the various formulations.

Secondly, when examining the effect of RC pressure it was found that a higher RC pressure led to an even higher $D_{50}$ value. A higher stress applied during RC causes higher densification of the primary powder into stronger granules (Patel et 
al., 2011). Therefore, ExMan-C granular material is larger in size following granulation.

\subsubsection{Tablet tensile strength and porosity}

Figure 6.3 shows the variation in tensile strength and porosity in tablets which contain varying ratios of fillers (MCC and mannitol) which have been produced by varying the processing parameters (granulation and tableting stress). Firstly, the effect of varying the formulation, namely the filler material, will be examined by comparing ExMCC-A and ExMan-A tablets. ExMCC-A tablets displayed lower tensile strength and porosity than ExMan-A tablets. MCC is a ductile material which is highly deformable (Mitra et al., 2016), whereas mannitol is brittle and does not deform as easily ( $\mathrm{Wu}$ and Sun, 2007). Therefore, a lower porosity in tablets containing an excess of MCC could occur due to MCC becoming highly deformed during application of stress, firstly during roller compaction and then during tableting, leading to a decreased in the void space within the tablet. On the other hand, mannitol may undergo less deformation during the applications of stress, therefore more void space within the tablet remains and consequently higher tablet porosity is observed.

Secondly, the effect of tableting stress on tablet tensile strength and porosity was investigated by compacting tablets at higher and lower forces of 25 or $15 \mathrm{kN}$ (ExMan-A and ExMan-B, respectively) using the excess mannitol formulation granules roller compacted at 20 bar pressure. ExMan-B tablets exhibited a lower tensile strength and higher porosity when compared to ExMan-A tablets. It has been reported in literature that, for a given material, a tablet with a low porosity can be expected to display high tensile strength (Sun, 2005). When a higher stress is applied 
during tableting, the granular material is more likely to be crushed into smaller fragments. Additionally, stronger inter-granular bonding occurs and consequently strong tablets are formed. Additionally less void space remains within the tablet due to the increased stress leading to greater reduction in the bulk material volume, and therefore a lower tablet porosity is observed (Mitra et al., 2016, Wu et al., 2006).

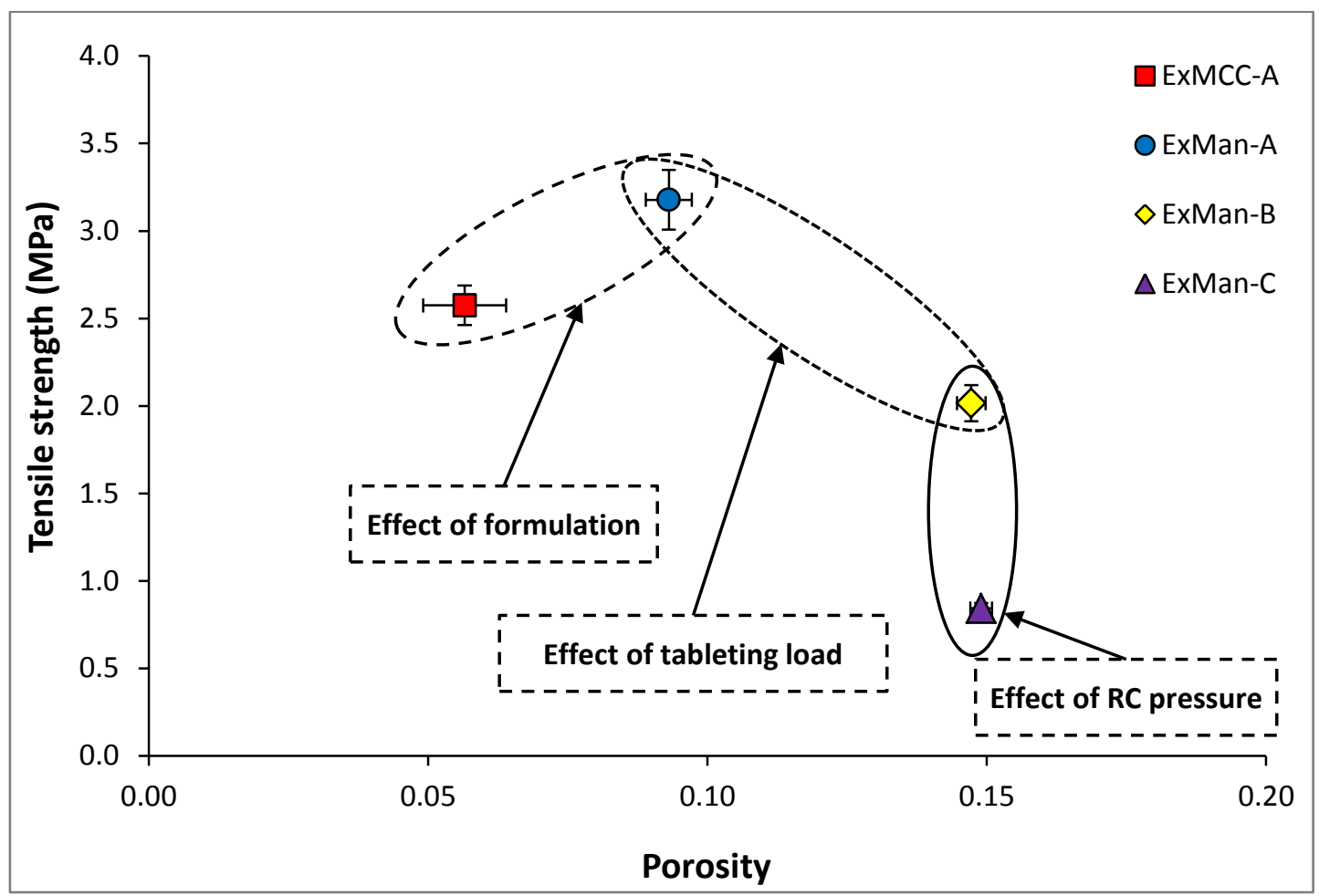

Figure 6.3 The tensile strength $\sigma_{\mathrm{T}}( \pm \mathrm{SD})$ and porosity $\varepsilon_{\mathrm{T}}( \pm \mathrm{SD})$ of tablets. Granules were produced using 20 or 50 bar roller compactor hydraulic pressure; the granules were then compressed using a tableting force of 15 or $25 \mathrm{kN}$.

Lastly, the effect of stress during granulation on tablet tensile strength and porosity was determined. Tablets were produced using the excess mannitol formulation granules produced using 20 and 50 bar ribbon pressure (ExMan-B and ExMan-C, respectively) during roller compaction and tableted using a constant force of $15 \mathrm{kN}$. Variation of ribbon pressure has a greater effect on tablet tensile strength than tablet porosity. Figure 6.3 shows that ExMan-B tablets and ExMan-C tablets 
displayed almost identical porosities, however they varied greatly in tensile strength. Weaker tablets were produced when a higher pressure was used during roller compactor granulation (50 bar). In literature it has been reported that when a high stress is applied the deformability of a material decreases which weakens the overall structure (Bultmann, 2002). However, from Section 3.4.4 it was determined that for binary mixtures the tableting stress applied to the material, and the extent of that stress, affects the consequent tablet properties differently depending on the RC stress previously applied to the material. So when a higher stress of 50 bar was applied during roller compaction, the primary powder is more strongly bonded within the granular material than the 20 bar granules. Therefore, a tableting force of $15 \mathrm{kN}$ is more likely to crush granular material roller compacted using 20 bar pressure into smaller fragments with a higher surface area. The tableting force is then more likely to cause stronger bonding of those smaller fragments to occur, leading to the 20 bar granule tablets being stronger.

\subsubsection{Standard USP analysis}

The disintegration times, which were recorded using the standard USP invitro testing equipment, are given in Table 6.3. From the USP analysis the average disintegration time ( $\mathrm{tavg}_{\mathrm{g}}$ ) and the time taken for the slowest tablet to disintegrate (tslow) is able to be determined. In terms of the effect of formulation, the USP analysis showed that the ExMCC-A tablets exhibit the longest disintegration time in the case of both the mean and slowest tablet disintegration time.

For the ExMan formulation, when looking at the effect of RC stress the ExMan-B and ExMan-C tablets disintegrated at almost identical times, which suggests that for this given formulation tablets of the same porosity, regardless of 
the RC stress applied to the material, will exhibit the same disintegration behaviour. When looking at the effect of tableting stress ExMan-A tablets displayed a marginally longer average disintegration time than the higher porosity ExMan-B tablets; but the tslow for the ExMan-A tablet was faster than the ExMan-B formulation tablet.

It can be observed that for the ExMan formulation the USP apparatus indicates that variation in the RC and tableting stresses leads to comparable disintegration times in the cases which are examined in this Chapter. There is hardly any variation in the disintegration times to distinguish the effect of the two stresses.

Table 6.3 Disintegration data obtained via USP analysis.

\begin{tabular}{|ccc|} 
& $\mathrm{t}_{\text {avg }}(\mathrm{min})$ & $\mathrm{t}_{\text {slow }}(\mathrm{min})$ \\
\hline ExMCC-A & $11.9 \pm 0.5$ & 12.9 \\
\hline ExMan-A & $5.2 \pm 0.8$ & 6.1 \\
\hline ExMan-B & $5.1 \pm 2.0$ & 8.5 \\
\hline ExMan-C & $5.1 \pm 1.6$ & 8.9 \\
\hline
\end{tabular}

\subsubsection{Flow Cell analysis}

\section{Imaging}

Examples of the binary images obtained with time during the disintegration of a tablet are shown at 10 min intervals in Figure 6.4. By conversion to a black and white image, the tablet and particles released from the tablet are more easily 
detected and quantified. The variation in tablet area, the number of particles released from the tablet and the average sizes of these particles were observed and quantified using a MATLAB code (see Appendix 18). The code recognises white pixels as the tablet and particles and uses these values to calculate the tablet $2 \mathrm{D}$ area and particle release number. The area was then normalised by dividing the tablet area at a given time by the initial tablet area. In the first image at $\mathrm{t}=0 \mathrm{~min}$ the initial tablet can be viewed with no evidence of disintegration. As time proceeds the tablet swelling and particle release can be observed. With time erosion of the tablet occurs, i.e. particle release and the tablet dissolves, a decrease in the tablet area is visible.

For ExMCC-A (Figure 6.4) the tablet swells significantly by $20 \mathrm{~min}$, and then slowly erodes from the edges (a possible explanation is given in Figure 5.4). This is due to the nature of MCC which tends to swell when it absorbs water. In the case of the ExMan-A and the ExMan-B tablets (Figure 6.4a and Figure 6.4b) after 20 min the tablet size appears to be equivalent. However, a difference in the remaining tablet area is more visible in the $20 \mathrm{~min}$ image where the ExMan-B tablet has eroded further than the ExMan-A. The fastest tablet erosion was observed in the ExMan-C tablet (Figure $6.4 \mathrm{~d}$ ) as the image at 20 min shows only a small area of remaining tablet after erosion has already occurred. 

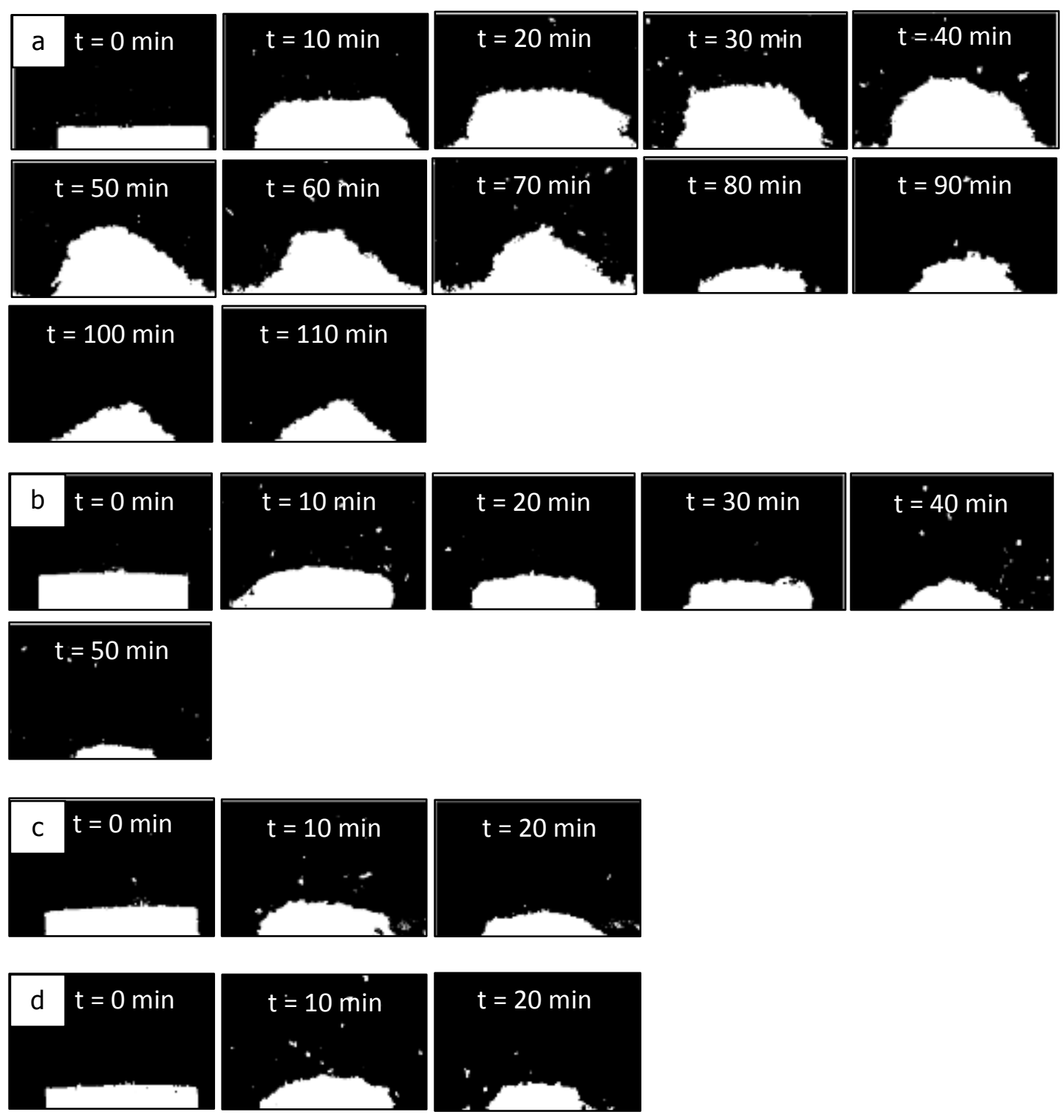

Figure 6.4 Binary images obtained during tablet disintegration at $\mathbf{1 0} \mathbf{~ m i n}$ intervals for tablets a) ExMCC-A; b) ExMan-A; c) ExMan-B; d) ExMan-C.

\section{Effect of filler content}

The effect of varying the filler content was examined in terms of the change in tablet area and the number of particles released with time (see Figure 6.5). Both batches of granules were produced by using 20 bar hydraulic pressure to compress the powder material via roller compactor granulation. A tableting force of $25 \mathrm{kN}$ 
was used to compact granules from the ExMCC and ExMan formulations respectively into tablets.

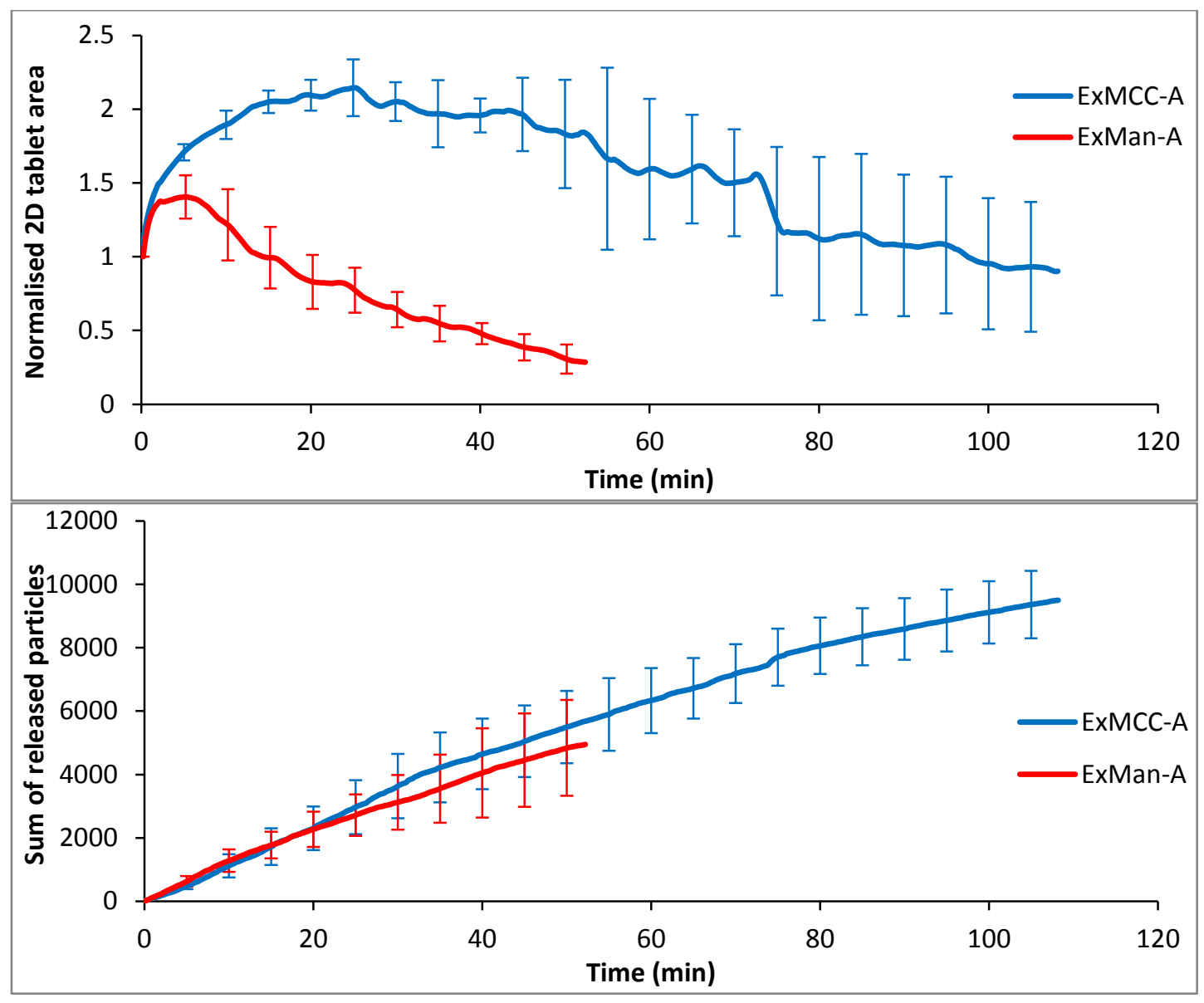

Figure 6.5 a) 2D tablet area vs time during the disintegration of tablets produced using granules compressed at 20 bar hydraulic pressure in a roller compactor. A tableting force of $25 \mathrm{kN}$ was used to compact the excess MCC and excess mannitol granules, respectively; b) the number of particles released for the aforementioned tablets.

Tablets containing an excess of MCC (ExMCC-A) swelled to the maximum tablet area after $24 \mathrm{~min}$. This is considerably delayed when compared to the tablet containing excess mannitol (ExMan-A) which swelled to the maximum tablet area at 5 min. ExMCC-A tablets also swelled to a higher maximum tablet area than ExManA tablets. This is attributable to MCC having an inherent affinity for absorbing more 
water than the available void space, causing the material to swell significantly (Ferrari et al., 1996).

ExMCC-A tablets also exhibited slower overall erosion time, i.e. reduction in tablet area, and released a greater number of particles than the ExMan-A tablets. ExMCC-A tablets were less porous than ExMan-A tablets which may prolong the disintegration timescale. It may also be suggested that higher tablet expansion could be due to slow water uptake, which leads to slow tablet breakage and therefore slow particle release. If the particles and granule within the tablet are strongly bonded together then, following the water uptake, the time taken for the bonding between the tablet materials to break may be slow. This in turn allows for more extensive tablet swelling to occur before particles begin to break away from the tablet. Additionally, mannitol is a highly soluble material, this factor may have influenced the faster disintegration observed in tablets containing an excess of mannitol. This is also supported by the standard USP analysis data (Table 6.3) which determined that the ExMan-A tablets disintegrated twice as fast as the ExMCC-A tablets. However, by using the Flow Cell method it was possible to determine that the ExMCC-A tablets swelled extensively, but in this case this did not mean that the tablet disintegrated more quickly. In actuality the tablet took longer to decrease in tablet size, and the particle release was comparable for both formulations.

The rate of particle release for both of the formulations appears to be almost identical. It should be taken into account that ExMan-A tablets contain a higher percentage of soluble material, so a lower number of particles were expected to be released. The size of the particles released may instead be larger in size for ExMCCA tablets. 


\section{Effect of ribbon pressure}

Tablets were produced using $15 \mathrm{kN}$ tableting force, containing excess mannitol granules which were roller compacted (RC) at either higher pressure (50 bar) or lower pressure (20 bar). The tablets were examined to determine the effect of RC pressure on tablet disintegration (see Figure 6.6). Tablets made using granules compressed at 50 bar roller compaction pressure (ExMan-C) swelled to a higher maximum area than tablet produced using 20 bar pressure (ExMan-B). Both ExMan$\mathrm{B}$ and ExMan-C tablets reached the maximum swelling area in approximately 2 min. Following the maximum area, the tablets erode at approximately the same rate.

Previously it was established that the two batches of tablets in question had almost identical porosity values, and only varied in tensile strength (see Figure 6.3). It can be suggested that the tablet swelling is related to how strongly the tablet is bonded, that tablet erosion is dependent on tablet porosity since the tablets produced by compressing the 20 and 50 bar granules had similar porosities. The results from the USP testing are in agreement with this as it also determined that the disintegration times for these tablets were almost identical (Table 6.3).

The tablets did vary in the particle release. The particle release rate was found to be slightly slower for the ExMan-C tablets than the ExMan-B tablets. However, the error was a little high so the trend is not clear. The size of the released particles may again play a role. The particles released from the tablets made using 20 bar are perhaps smaller in size than in the 50 bar, but more particles are observed overall. 


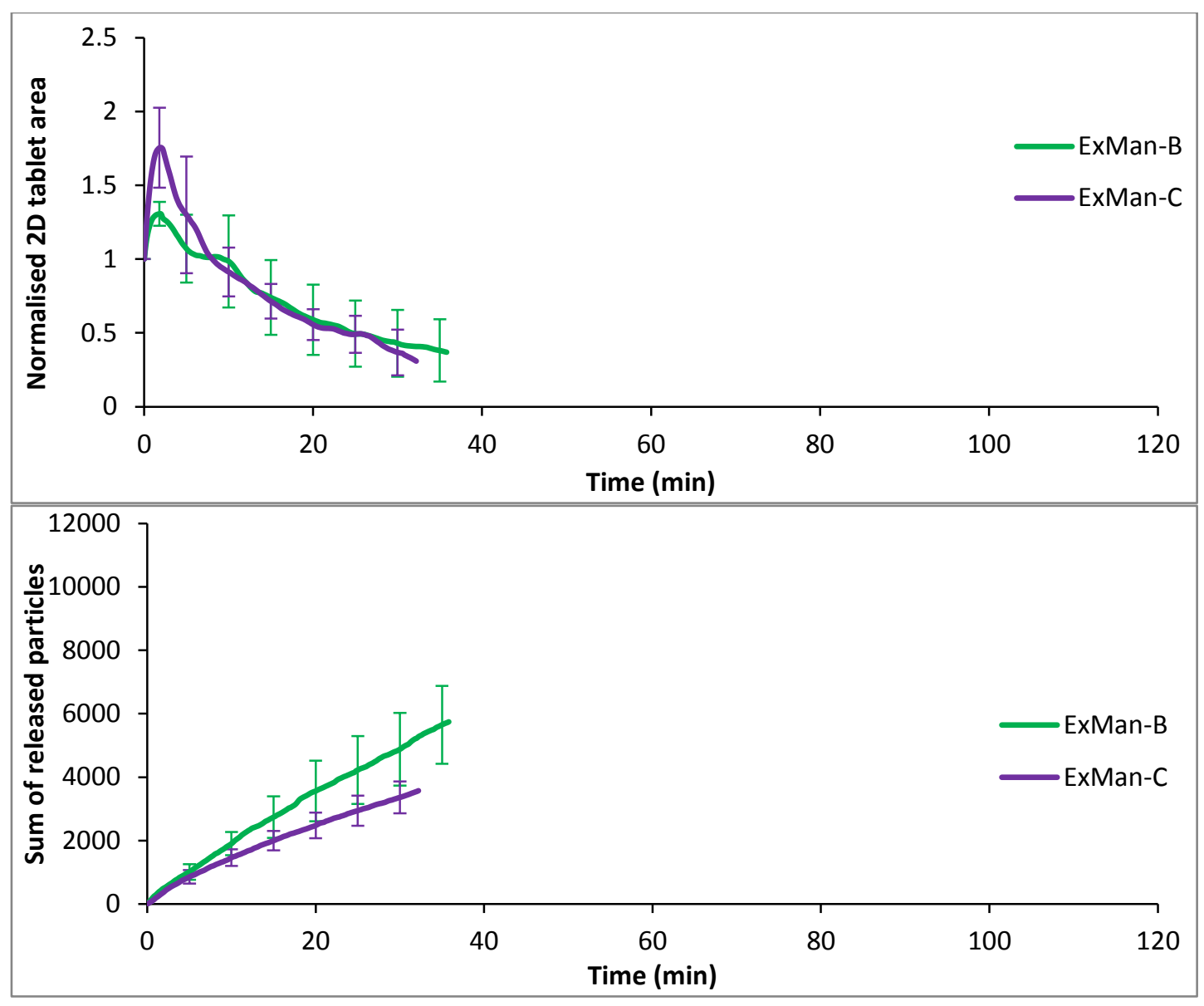

Figure 6.6 a) 2D tablet area vs time during the disintegration of tablets produced using granules compressed using 20 and 50 bar roller compactor pressure, compacted at a tableting force of $15 \mathrm{kN}$. b) The number of particles released for the aforementioned tablets.

\section{Effect of tableting force}

Tablets were produced using the excess mannitol formulation by compacting 20 bar granular material using either 15 or $25 \mathrm{kN}$ tableting force to determine the effect of varying tableting force. A significant difference was not observed using the USP standard disintegration testing equipment (Table 6.3). But by using the Flow Cell method the change in the 2D tablet area with time (see Figure 6.7) showed that ExMan-A tablets swelled slightly more and at a slower rate than the ExMan-B tablets. The same granular material was used for tableting; therefore, it can be 
assumed that the variation in the extent of swelling is due to the compaction force used during tableting and the consequent tablet tensile strength and porosity values. The ExMan-A tablets swelled in size to a greater extent, but the time take to reach the maximum swelling area was also greater. The erosion of the tablet, i.e. the reduction in size of the tablet, following swelling was also delayed for ExMan-A tablets. This may be due to the bonding within the tablet being stronger and therefore the time taken for these bonds to break following water uptake may be delayed. This leads to significant tablet swelling, due to the MCC expanding following water absorption, before the tablet begins to decrease in area. This may also be a relaxation phenomenon where a greater change in the tablet area is seen following a greater stress applied during tableting.

Faster disintegration times were observed with ExMan-B tablets, and correspondingly a faster particle release rate. A slower particle release was seen in the case of the less porous ExMan-A tablets. In addition, the overall number of particles released at a given time was lower for the ExMan-A tablets. A possible reasoning for this phenomenon could be due to the slower tablet disintegration and release of the particles in these tablets; the soluble materials within the particles may dissolve into solution before being detected by the imaging method and therefore fewer particles are observed. 


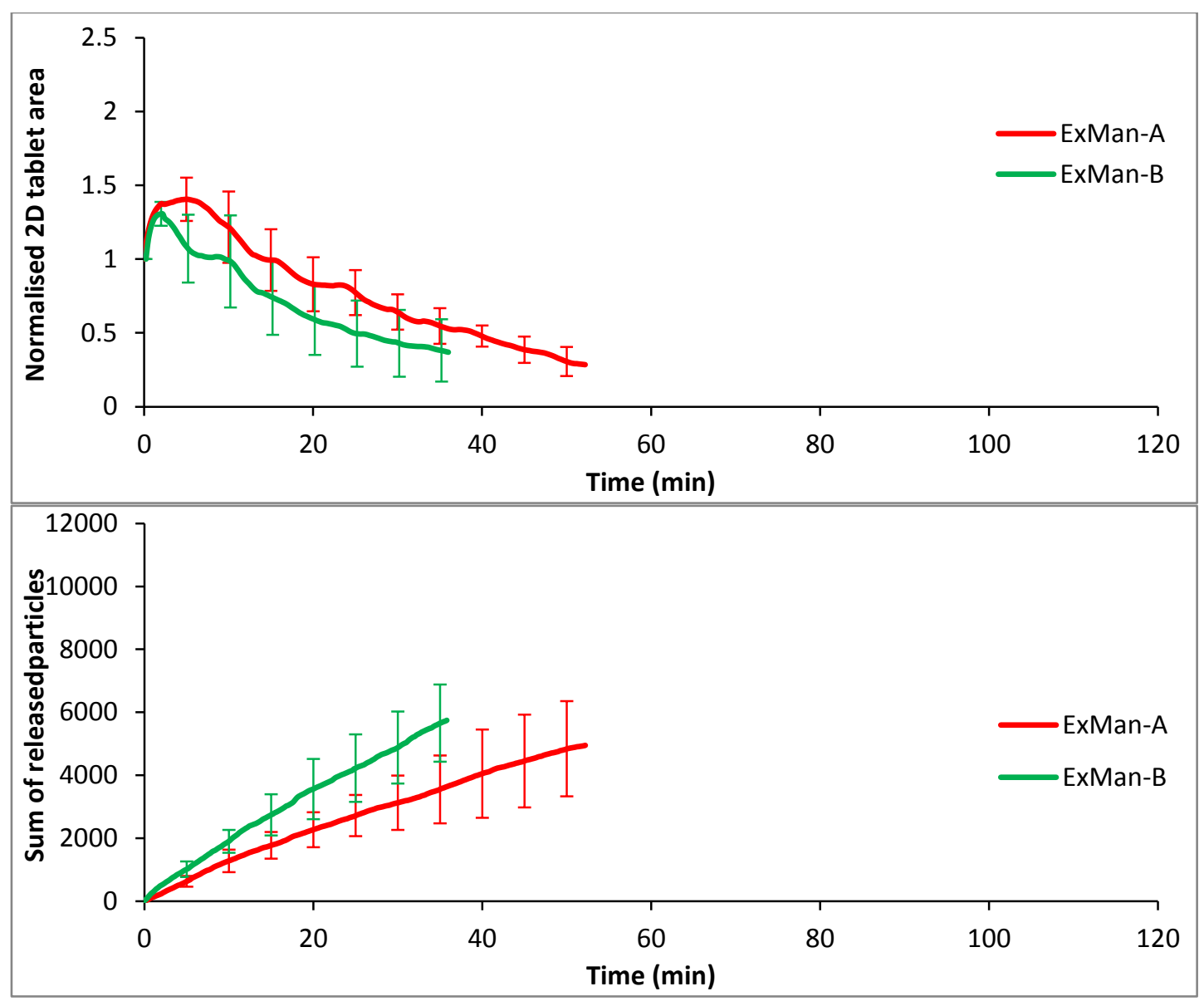

Figure 6.7 a) 2D tablet area vs time during the disintegration of tablets produced using granules compressed using 20 bar roller compactor pressure, compacted at a tableting force of 25 or $15 \mathrm{kN} \mathrm{b}$ ) The number of particles released for the aforementioned tablets.

\section{Size of released particles}

In addition to being able to detect the tablet area and the number of particles released with time, the Flow Cell is also capable of monitoring the size of the released particles. However, the error for these data points was also quite high, as also seen with the LIXELL measurement (see Section 4.4.1).

The $\mathrm{D}_{10}, \mathrm{D}_{50}$ and $\mathrm{D}_{90}$ values for ExMCC-A tablets with time are shown in Figure 6.8. Overall, the ExMCC-A tablets released the largest particles throughout the disintegration. This is due to the high percentage of MCC. MCC swells on 
contact with water and is insoluble in water; therefore the Flow Cell method detected the release of the swollen MCC particulate.

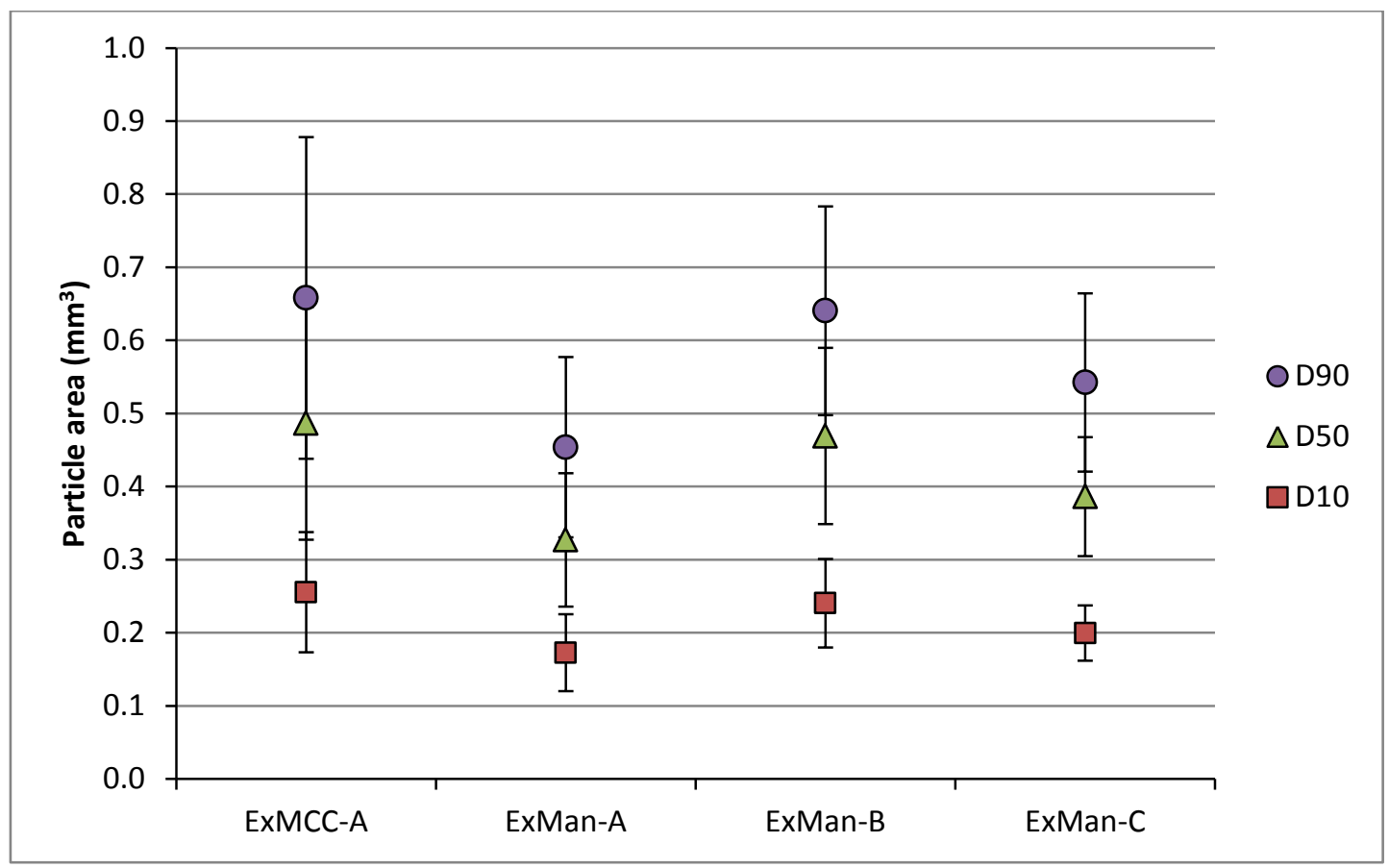

Figure 6.8 Average $D_{10}, D_{50}$ and $D_{90}$ values for the particles released from the tablets with time.

When considering the effect of variation in the formulation ExMan-A tablets released smaller particles than the ExMCC-A tablets. This may be due to the lower percentage of $\mathrm{MCC}$ in the formulation, meaning that the percentage of larger swollen MCC particles being detected is lower. From Figure 6.5 it was observed that the number of particles released was almost identical for ExMCC-A and ExMan-A tablets therefore the size of the particles is likely due to the increased MCC content in ExMCC-A tablets.

When comparing the effect of RC pressure, ExMan-C tablets released slightly smaller particles than ExMan-B. From Figure 6.6 it was determined that the number of particles releases was also slightly higher for ExMan-B tablets which was not 
expected. For these conditions it was expected ExMan-C tablets would release a lower number of larger particles due to the granules being larger in size and the tableting stress being insufficient to crush and bond them. However a lower number of slightly smaller particle were release, therefore this may be experimental error or there is another factor at play.

When considering the effect of tableting force on the size of the particles released, the ExMan-B tablets released larger particles than the ExMan-A tablets. This may be due to the tableting force of $25 \mathrm{kN}$ in the case of ExMan-A tablets being sufficiently high enough so that the granular material is crushed and forms strong bonds. Additionally, the porosity is also lower, meaning that fewer pores also exist. Water influx into the tablet occurs only in the limited weaker points in the tablet so the tablet disintegration leads to the release of larger fragments.

\subsection{Conclusion}

By using a particle imaging method more quantifiable information regarding the mechanisms of disintegration, including tablet swelling and particle release, was gained. The excipients of a tablet, particularly the filler which constitutes the bulk of the formulation, drastically affect the disintegration behaviour. Swelling is observed with the presence of $\mathrm{MCC}$ in the tablet formulation; the degree of swelling is directly dependent on the percentage of MCC. The ExMCC-A tablet formulation reached a higher maximum tablet area, whereas the ExMan-A tablets swelled but to a lower extent. The particle release rates were found to be almost identical, but ExMCC-A tablets released larger particles overall.

The processing parameters used to produce tablets also play a part in how a tablet will erode. It is important to consider the first stress applied during RC and 
the second stress which occurs during tableting. It was found that the swelling and disintegration trends were comparable for the tablets made using granules produced at 20 and 50 bar using both the USP method and the Flow Cell method, due to the tablets having almost equivalent porosities. However, by using the Flow Cell method it was possible to differentiate between the particle release rates for the two conditions. In brief, it was determined that the granule size distribution influences the particle release rate and it was reinforced that the porosity of a tablet influences the disintegration behaviour in terms of tablet swelling and erosion, however there are many other factors which also have an influence.

In the case of the tablets made using 15 or $25 \mathrm{kN}$ tableting force, a greater increase in tablet area and slower tablet erosion was observed for the latter tablet, which indicates a relaxation phenomenon occurrence. A slower rate of particle release was also detected for tablets compressed at $25 \mathrm{kN}$, and overall a lower number of total particles released was observed. This suggests that the slower rate of release combined with the slower disintegration of the tablet leads to the soluble material in the tablet, mainly mannitol, dissolving into solution before being detected by the imaging method.

Overall it was established that the particle release is highly dependent on the processing conditions used to produce the tablet. The particle release is dependent on whether the granular material is crushed during tableting, and to what extent. Additionally, whether or not the stress applied during tableting causes strong bonding within the tablet also affects the size of the particles released. 


\section{Chapter 7. Conclusions and Future Work}

\subsection{Conclusions}

This research set out to determine the effect of variation in the formulation and processing parameters on the tablet properties, namely the tablet compactibility and the tablet disintegration/dissolution behaviour.

This study showed that the compactibility of a tablet is dependent on the two main stresses used during the production of a tablet; the roller compaction stress and the tableting stress. The roller compaction stress used during granulation determines the granular porosity. If the tableting stress applied to the granules is higher than the stress applied during granulation, then the granular material has the potential to be partially crushed. If the tableting stress is significantly higher than the RC stress, then the granular material more of the granule is crushed and also forms strong particle-particle bonds within the tablet. In literature this is termed as a "loss in compressibility", which suggests that the material loses the ability to form strong tablets following high granulation stress. However, this is not the case; a more highly roller compacted granule requires a higher tableting stress to produce tablets of the same strength as a less compacted granule. By using X-ray tomography and compactibility studies it was confirmed that the granular integrity within the tablet varied depending on the RC and tableting stresses used. In terms of the material, MCC was found to produce the strongest tablets in every case. 
Three different analysis methods were employed to determine the tablet disintegration behaviour. The LIXELL, Flow Cell and USP analysis methods were used to compare the disintegration behaviour for binary mixtures and placebo formulations, which were produced using varying granulation and tableting stresses. Two imaging techniques were compared to the standard USP method. This was done to determine which disintegration analysis method is able to provide more useful, quantifiable information to better understand the disintegration process. Additionally, ${ }^{1} \mathrm{H}$ NMR was used in conjunction with the LIXELL analysis as a method of monitoring mannitol dissolution.

The LIXELL method was able to detect differences in the cumulative particle release depending on the tablet formulation. But due to the nature of the LIXELL measurement being semi on-line, i.e. the detection occurs away from the disintegrating tablet, the data was too scattered to confirm with any certainty the validity of the results which were obtained. Additionally, it must be considered that the LIXELL technique subjects the tablet to mechanical stress due to the paddle stirrer. This may affect the results as a level of inaccuracy is introduced.

The ${ }^{1} \mathrm{H}$ NMR analysis was successfully able to determine the mannitol concentration in solution with time. It was found that a higher tableting stress used led to slower mannitol dissolution. However, the technique was found to be inherently inaccurate due to the method being off-line. It has to be considered that the concentration which was calculated included both the dissolved and undissolved mannitol as the undissolved mannitol had time to enter into solution.

The USP method was able to distinguish different disintegration times when the formulation was varied, where tablets containing a higher percentage of MCC disintegrated more slowly. However, when the formulation containing an excess of 
mannitol was analysed, the change in granulation and tableting parameters did not affect the disintegration times as distinctly.

It was found that the Flow Cell method was able to detect changes in the disintegration behaviour when the processing parameters and formulation were altered, whereas the USP method was not. As the Flow Cell is an on-line imaging approach no information is lost, and it is possible to detect the change in tablet area as well as the particle release. Whereas the USP method was not able to detect these variations and determined that changes in the processing parameters had no effect on the disintegration time. Therefore it was confirmed that the Flow Cell is a more viable method in tablet disintegration analysis.

To conclude, it was possible to visualise tablet disintegration using two different imagining analysis methods, but a more on-line method was found to be more precise. This meant that additional information was available which adds to the understanding of the processes occurring during disintegration, in terms of the particle release and tablet area, are affected by changes in the tablet formulation and processing parameters. This knowledge will allow for better and more efficient tablet design.

\subsection{Future Work}

From this research it was found that the Flow Cell is a viable on-line method for the analysis of pharmaceutical tablet disintegration, which is capable of distinguishing the effect of changes in processing parameters. The addition of a Refractive Index probe to the Flow Cell method will allow for the simultaneous examination of tablet disintegration as well as dissolution. Changes in the refractive index occur due to the dissolution of a soluble component, which in turn changes 
the density of the dissolution medium. Unlike conductivity meters which can only detect ionic components, the refractive index is able to detect any soluble materials as they dissolve in real-time.

This study has found that the stresses applied to a material affect the tablet disintegration in various ways. It would be useful to link the mechanical properties of the materials to the tablet compactibility and disintegration behaviour observed using the imaging methods.

Higher resolution X-ray, such as synchrotron, measurements also need to be carried out to quantify the particle size distribution which exists within a tablet depending on the processing parameters used. A higher stress applied during tableting is expected to cause smaller particles to be present within the tablet if a low RC pressure granule is present. Additionally, the granular structure needs to be examined to monitor the granular porosity and the intra-granular tablet porosity with increasing tableting stress, with the tableting stress significantly surpassing the stress applied to the material during roller compaction. This will provide insight into the granule breakage which occurs during tableting, and it can also be determined whether the "loss in compressibility" is able to be overcome with sufficient tableting stress. 


\section{References}

ABDELbARY, G., EOUANI, C., PRINDERRE, P., JOACHIM, J., REYNIER, J. \& PICCERELLE, P. 2005. Determination of the in vitro disintegration profile of rapidly disintegrating tablets and correlation with oral disintegration. International Journal of Pharmaceutics, 292, 29-41.

ADOLFSSON, Å. \& NYSTRÖM, C. 1996. Tablet strength, porosity, elasticity and solid state structure of tablets compressed at high loads. International Journal of Pharmaceutics, 132, 95-106.

ALLESØ, M., HOLM, R. \& HOLM, P. 2016. Roller compaction scale-up using roll width as scale factor and laser-based determined ribbon porosity as critical material attribute. European Journal of Pharmaceutical Sciences, 87, 69-78.

AZARMI, S., ROA, W. \& LÖBENBERG, R. 2007. Current perspectives in dissolution testing of conventional and novel dosage forms. International Journal of Pharmaceutics, 328, 12-21.

BAI, G., WANG, Y. \& ARMENANTE, P. M. 2011. Velocity profiles and shear strain rate variability in the USP Dissolution Testing Apparatus 2 at different impeller agitation speeds. International Journal of Pharmaceutics, 403, 1-14.

BUGGINS, T. R., DICKINSON, P. A. \& TAYLOR, G. 2007. The effects of pharmaceutical excipients on drug disposition. Advanced Drug Delivery Reviews, 59, 1482-1503.

BULTMANN, J. M. 2002. Multiple compaction of microcrystalline cellulose in a roller compactor. European Journal of Pharmaceutics and Biopharmaceutics, 54, 5964.

BUSIGNIES, V., LECLERC, B., PORION, P., EVESQUE, P., COUARRAZE, G. \& TCHORELOFF, P. 2006. Quantitative measurements of localized density variations in cylindrical tablets using X-ray microtomography. European Journal of Pharmaceutics and Biopharmaceutics, 64, 38-50. 
CARAMELlA, C., COLOMBO, P., CONTE, U., FERRARI, F., GAZZANIGA, A., LAMANNA, A. \& PEPPAS, N. A. 1988. A physical analysis of the phenomenon of tablet disintegration. International Journal of Pharmaceutics, 44, 177-186.

CHANG, C. K., ALVAREZ-NUNEZ, F. A., RINELLA JR, J. V., MAGNUSSON, L.-E. \& SUEDA, K. 2008. Roller Compaction, Granulation and Capsule Product Dissolution of Drug Formulations Containing a Lactose or Mannitol Filler, Starch, and Talc. AAPS PharmSciTech, 9, 597-604.

COLOMBO, P., CONTE, U., CARAMELLA, C., LA MANNA, A. \& GEDDO, M. 1984. Disintegrating force as a new formulation parameter. Journal of Pharmaceutical Sciences, 73, 701-705.

COLOMBO, P., CONTE, U., CARAMELLA, C., LA MANNA, A., GUYOTHERMANN, A. M. \& RINGARD, J. 1980. Disintegrating power of tablets. Farmaco Prat, 35, 391-402.

COOMBES, S. R., HUGHES, L. P., PHILLIPS, A. R. \& WREN, S. A. C. 2014. Proton NMR: A New Tool for Understanding Dissolution. Analytical Chemistry, 86, 2474-2480.

COOPER, J. \& REES, J. E. 1972. Tableting research and technology. Journal of Pharmaceutical Sciences, 61, 1511-1555.

DELALONDE, M. \& RUIZ, T. 2008. Dissolution of pharmaceutical tablets: The influence of penetration and drainage of interstitial fluids. Chemical Engineering and Processing: Process Intensification, 47, 370-376.

DESAI, D. S., RANADIVE, S. A., LOZANO, R. \& VARIA, S. A. 1996. Dissolution instability of encapsulated marketed tablets. International Journal of Pharmaceutics, 144, 153-158.

DESAI, P. M., LIEW, C. V. \& HENG, P. W. S. 2016. Review of Disintegrants and the Disintegration Phenomena. Journal of Pharmaceutical Sciences, 105, 2545-2555.

DONAUER, N. \& LÖBENBERG, R. 2007. A mini review of scientific and pharmacopeial requirements for the disintegration test. International Journal of Pharmaceutics, 345, 2-8. 
FARBER, L., HAPGOOD, K. P., MICHAELS, J. N., FU, X.-Y., MEYER, R., JOHNSON, M.-A. \& LI, F. 2008. Unified compaction curve model for tensile strength of tablets made by roller compaction and direct compression. International Journal of Pharmaceutics, 346, 17-24.

FASSIHI, A. R. 1986. Mechanisms of disintegration and compactibility of disintegrants in a direct compression system. International Journal of Pharmaceutics, 32, 93-96.

FELL, J. T. \& NEWTON, J. M. 1968. The tensile strength of lactose tablets. Journal of Pharmacy and Pharmacology, 20, 657-659.

FERRARI, F., BERTONI, M., BONFERONI, M. C., ROSSI, S., CARAMELLA, C. \& NYSTRÖM, C. 1996. Investigation on bonding and disintegration properties of pharmaceutical materials. International Journal of Pharmaceutics, 136, 71-79.

GAD, S. C. 2008. Pharmaceutical Manufacturing Handbook: Production and Processes, Wiley.

GUIGON, P., SIMON, O., SALEH, K., BINDHUMADHAVAN, G., J. ADAMS, M. \& SEVILLE, J. P. K. 2007. Chapter 5 Roll pressing. In: A.D. SALMAN, M. J. H. \& SEVILLE, J. P. K. (eds.) Handbook of Powder Technology. Elsevier Science B.V.

HADŽOVIĆ, E., BETZ, G., HADŽIDEDIĆ, Š., EL-ARINI, S. K. \& LEUENBERGER, H. 2011. Investigation of compressibility and compactibility parameters of roller compacted Theophylline and its binary mixtures. International Journal of Pharmaceutics, 416, 97-103.

HATTORI, Y. \& OTSUKA, M. 2011. NIR spectroscopic study of the dissolution process in pharmaceutical tablets. Vibrational Spectroscopy, 57, 275-281.

HE, X., SECREAST, P. J. \& AMIDON, G. E. 2007. Mechanistic Study of the Effect of Roller Compaction and Lubricant on Tablet Mechanical Strength. Journal of Pharmaceutical Sciences, 96, 1342-1355.

HERTING, M. G. \& KLEINEBUDDE, P. 2007. Roll compaction/dry granulation: Effect of raw material particle size on granule and tablet properties. International Journal of Pharmaceutics, 338, 110-118. 
HERTING, M. G. \& KLEINEBUDDE, P. 2008. Studies on the reduction of tensile strength of tablets after roll compaction/dry granulation. European Journal of Pharmaceutics and Biopharmaceutics, 70, 372-379.

IBRAHIM, H. G. \& SALLAM, E.-S. 1993. Dissolution from disintegrating tablet: separate contributions from granules and primary drug particles considered. International Journal of Pharmaceutics, 93, 111-120.

INGHELBRECHT, S., REMON, J.-P., FERNANDES DE AGUIAR, P., WALCZAK, B., MASSART, D., VAN DE VELDE, F., DE BAETS, P., VERMEERSCH, H. \& DE BACKER, P. 1997. Instrumentation of a roll compactor and the evaluation of the parameter settings by neural networks. International Journal of Pharmaceutics, 148, 103-115.

INGHELBRECHT, S. \& REMON, J. P. 1998a. Reducing dust and improving granule and tablet quality in the roller compaction process. International Journal of Pharmaceutics, 171, 195-206.

INGHELBRECHT, S. \& REMON, J. P. 1998b. Roller compaction and tableting of microcrystalline cellulose/drug mixtures. International Journal of Pharmaceutics, $161,215-224$.

JIVRAJ, M., MARTINI, L. G. \& THOMSON, C. M. 2000. An overview of the different excipients useful for the direct compression of tablets. Pharmaceutical Science $\mathcal{E}$ Technology Today, 3, 58-63.

JOHANSON, J. R. 1965. A Rolling Theory for Granular Solids. Journal of Applied Mechanics, 32, 842-848.

JOHANSSON, B., WIKBERG, M., EK, R. \& ALDERBORN, G. 1995. Compression behaviour and compactability of microcrystalline cellulose pellets in relationship to their pore structure and mechanical properties. International Journal of Pharmaceutics, 117, 57-73.

KATZHENDLER, I., HOFFMAN, A., GOLDBERGER, A. \& FRIEDMAN, M. 1997. Modeling of drug release from erodible tablets. Journal of Pharmaceutical Sciences, 86, 110-115.

KAZEMI, P., KHALID, M. H., PÉREZ GAGO, A., KLEINEBUDDE, P., JACHOWICZ, R., SZLĘK, J. \& MENDYK, A. 2017. Effect of roll compaction on 
granule size distribution of microcrystalline cellulose-mannitol mixtures: computational intelligence modeling and parametric analysis. Drug Design, Development and Therapy, 11, 241-251.

KHORASANI, M., AMIGO, J. M., BERTELSEN, P., SUN, C. C. \& RANTANEN, J. 2016. Process optimization of dry granulation based tableting line: Extracting physical material characteristics from granules, ribbons and tablets using near-IR (NIR) spectroscopic measurement. Powder Technology, 300, 120-125.

KLEINEBUDDE, P. 2004. Roll compaction/dry granulation: pharmaceutical applications. European Journal of Pharmaceutics and Biopharmaceutics, 58, 317326.

LEUENBERGER, H. 1982. The compressibility and compactibility of powder systems. International Journal of Pharmaceutics, 12, 41-55.

LI, Q., RUDOLPH, V., WEIGL, B. \& EARL, A. 2004. Interparticle van der Waals force in powder flowability and compactibility. International Journal of Pharmaceutics, 280, 77-93.

LOFTSSON, T. 2015. Excipient pharmacokinetics and profiling. International Journal of Pharmaceutics, 480, 48-54.

LOWENTHAL, W. 1972. Disintegration of tablets. Journal of Pharmaceutical Sciences, 61, 1695-1711.

LUGINBÜHL, R. \& LEUENBERGER, H. 1994. Use of percolation theory to interpret water uptake, disintegration time and intrinsic dissolution rate of tablets consisting of binary mixtures. Pharmaceutica Acta Helvetiae, 69, 127-134.

MERKKU, P., LINDQVIST, A.-S., LEIVISKA, K. \& YLIRUUSI, J. 1994. Influence of granulation and compression process variables on flow rate of granules and on tablet properties, with special reference to weight variation. International Journal of Pharmaceutics, 102, 117-125.

MESNIER, X., ALTHAUS, T. O., FORNY, L., NIEDERREITER, G., PALZER, S., HOUNSLOW, M. J. \& SALMAN, A. D. 2013. A novel method to quantify tablet disintegration. Powder Technology, 238, 27-34. 
MIGUÉLEZ-MORÁN, A. M., WU, C. Y. \& SEVILLE, J. P. K. 2008. The effect of lubrication on density distributions of roller compacted ribbons. International Journal of Pharmaceutics, 362, 52-59.

MILLER, T. A. \& YORK, P. 1988. Pharmaceutical tablet lubrication. International Journal of Pharmaceutics, 41, 1-19.

MITRA, B., HILDEN, J. \& LITSTER, J. D. 2015. Novel use of monodisperse granules to deconvolute impacts of granule size versus granule solid fraction on tablet tensile strength. Advanced Powder Technology, 26, 553-562.

MITRA, B., HILDEN, J. \& LITSTER, J. D. 2016. Compaction mechanics of plastically deformable dry granules. Powder Technology, 291, 328-336.

MOSIG, J. \& KLEINEBUDDE, P. 2015. Critical Evaluation of Root Causes of the Reduced Compactability after Roll Compaction/Dry Granulation. Journal of Pharmaceutical Sciences, 104, 1108-1118.

NOTT, K. P. 2010. Magnetic resonance imaging of tablet dissolution. Eur J Pharm Biopharm, 74, 78-83.

OHREM, H. L., SCHORNICK, E., KALIVODA, A. \& OGNIBENE, R. 2014. Why is mannitol becoming more and more popular as a pharmaceutical excipient in solid dosage forms? Pharmaceutical Development and Technology, 19, 257-262.

OTSUKA, M., YAMANE, I. \& MATSUDA, Y. 2004. Effects of lubricant mixing on compression properties of various kinds of direct compression excipients and physical properties of the tablets. Advanced Powder Technology, 15, 477-493.

PABARI, R. M. \& RAMTOOLA, Z. 2012. Effect of a Disintegration Mechanism on Wetting, Water Absorption, and Disintegration Time of Orodispersible Tablets. Journal of Young Pharmacists, 4, 157-163.

PATEL, S., DAHIYA, S., CALVIN SUN, C. \& BANSAL, A. K. 2011. Understanding Size Enlargement and Hardening of Granules on Tabletability of Unlubricated Granules Prepared by Dry Granulation. Journal of Pharmaceutical Sciences, 100, 758-766. 
PEPPAS, N. A. \& COLOMBO, P. 1989. Development of disintegration forces during water penetration in porous pharmaceutical systems. Journal of Controlled Release, 10, 245-250.

PEPPAS, N. A., COLOMBO, P., CONTE, U., GAZZANIGA, A., CARAMELLA, C. \& FERRARI, F. 1989. Energetics of tablet disintegration. International Journal of Pharmaceutics, 51, 77-83.

PEREZ-GANDARILLAS, L., MAZOR, A., SOURIOU, D., LECOQ, O. \& MICHRAFY, A. 2015. Compaction behaviour of dry granulated binary mixtures. Powder Technology, 285, 62-67.

PEREZ-GANDARILLAS, L., PEREZ-GAGO, A., MAZOR, A., KLEINEBUDDE, P., LECOQ, O. \& MICHRAFY, A. 2016. Effect of roll-compaction and milling conditions on granules and tablet properties. European Journal of Pharmaceutics and Biopharmaceutics, 106, 38-49.

PÉREZ GAGO, A., REYNOLDS, G. \& KLEINEBUDDE, P. 2017. Impact of roll compactor scale on ribbon density. Powder Technology.

PIFFERI, G., SANTORO, P. \& PEDRANI, M. 1999. Quality and functionality of excipients. Il Farmaco, 54, 1-14.

PITT, K. \& SINKA, C. 2007. Chapter 16 Tabletting. In: A.D. SALMAN, M. J. H. \& SEVILLE, J. P. K. (eds.) Handbook of Powder Technology. Elsevier Science B.V.

QUODBACH, J. \& KLEINEBUDDE, P. 2014. A New Apparatus for Real-Time Assessment of the Particle Size Distribution of Disintegrating Tablets. Journal of Pharmaceutical Sciences, 103, 3657-3665.

QUODBACH, J., MOUSSAVI, A., TAMMER, R., FRAHM, J. \& KLEINEBUDDE, P. 2014a. Assessment of disintegrant efficacy with fractal dimensions from realtime MRI. International Journal of Pharmaceutics, 475, 605-612.

QUODBACH, J., MOUSSAVI, A., TAMMER, R., FRAHM, J. \& KLEINEBUDDE, P. 2014b. Tablet Disintegration Studied by High-Resolution Real-Time Magnetic Resonance Imaging. Journal of Pharmaceutical Sciences, 103, 249-255.

RAZAVI, S. M., CALLEGARI, G., DRAZER, G. \& CUITIÑO, A. M. 2016. Toward predicting tensile strength of pharmaceutical tablets by ultrasound 
measurement in continuous manufacturing. International Journal of Pharmaceutics, 507, 83-89.

REYNOLDS, G., INGALE, R., ROBERTS, R., KOTHARI, S. \& GURURAJAN, B. 2010. Practical application of roller compaction process modeling. Computers $\mathcal{E}$ Chemical Engineering, 34, 1049-1057.

RÖST, M. \& QUIST, P. O. 2003. Dissolution of USP prednisone calibrator tablets: Effects of stirring conditions and particle size distribution. Journal of Pharmaceutical and Biomedical Analysis, 31, 1129-1143.

ROTT, N. 1990. Note on the History of the Reynolds Number. Annual Review of Fluid Mechanics, 22, 1-12.

ROWE, R. C., SHESKEY, P. J., QUINN, M. E. \& ASSOCIATION, A. P. 2009. Handbook of Pharmaceutical Excipients, Pharmaceutical Press.

SAVITZKY, A. \& GOLAY, M. J. E. 1964. Smoothing and Differentiation of Data by Simplified Least Squares Procedures. Analytical Chemistry, 36, 1627-1639.

SCHMIDTKE, R., SCHRÖDER, D., MENTH, J., STAAB, A., BRAUN, M. \& WAGNER, K. G. 2017. Prediction of solid fraction from powder mixtures based on single component compression analysis. International Journal of Pharmaceutics, 523, 366-375.

SHIKO, G., GLADDEN, L. F., SEDERMAN, A. J., CONNOLLY, P. C. \& BUTLER, J. M. 2011. MRI Studies of the Hydrodynamics in a USP 4 Dissolution Testing Cell. Journal of Pharmaceutical Sciences, 100, 976-991.

SINKA, I. C., MOTAZEDIAN, F., COCKS, A. C. F. \& PITT, K. G. 2009. The effect of processing parameters on pharmaceutical tablet properties. Powder Technology, $189,276-284$.

SOUIHI, N., REYNOLDS, G., TAJAROBI, P., WIKSTRÖM, H., HAEFFLER, G., JOSEFSON, M. \& TRYGG, J. 2015. Roll compaction process modeling: Transfer between equipment and impact of process parameters. International Journal of Pharmaceutics, 484, 192-206. 
SUN, C. \& HIMMELSPACH, M. W. 2006. Reduced tabletability of roller compacted granules as a result of granule size enlargement. Journal of Pharmaceutical Sciences, 95, 200-206.

SUN, C. C. 2005. Quantifying errors in tableting data analysis using the Ryshkewitch equation due to inaccurate true density. Journal of Pharmaceutical Sciences, 94, 2061-2068.

SUNADA, H. \& BI, Y. 2002. Preparation, evaluation and optimization of rapidly disintegrating tablets. Powder Technology, 122, 188-198.

THOORENS, G., KRIER, F., LECLERCQ, B., CARLIN, B. \& EVRARD, B. 2014. Microcrystalline cellulose, a direct compression binder in a quality by design environment-A review. International Journal of Pharmaceutics, 473, 64-72.

TYE, C. K., SUN, C. \& AMIDON, G. E. 2005. Evaluation of the effects of tableting speed on the relationships between compaction pressure, tablet tensile strength, and tablet solid fraction. Journal of Pharmaceutical Sciences, 94, 465472.

VAN DER VOORT MAARSCHALK, K., ZUURMAN, K., VROMANS, H., BOLHUIS, G. K. \& LERK, C. F. 1996. Porosity expansion of tablets as a result of bonding and deformation of particulate solids. International Journal of Pharmaceutics, 140, 185-193.

WAGNER, C. M., PEIN, M. \& BREITKREUTZ, J. 2013. Roll compaction of mannitol: Compactability study of crystalline and spray-dried grades. International Journal of Pharmaceutics, 453, 416-422.

WAGNER, C. M., PEIN, M. \& BREITKREUTZ, J. 2015. Roll compaction of granulated mannitol grades and the unprocessed crystalline delta-polymorph. Powder Technology, 270, 470-475.

WAGNER, J. G. 1969. Interpretation of percent dissolved-time plots derived from in vitro testing of conventional tablets and capsules. Journal of Pharmaceutical Sciences, 58, 1253-1257.

WAN, L. S. C. \& PRASAD, K. P. P. 1988. Effect of microcrystalline cellulose and cross-linked sodium carboxymethylcellulose on the properties of tablets with methylcellulose as a binder. International Journal of Pharmaceutics, 41, 159-167. 
WANG, J., WEN, H. \& DESAI, D. 2010. Lubrication in tablet formulations. European Journal of Pharmaceutics and Biopharmaceutics, 75, 1-15.

WILLIAMS, R. L. 2006. Official USP Reference Standards: Metrology concepts, overview, and scientific issues and opportunities. Journal of Pharmaceutical and Biomedical Analysis, 40, 3-15.

WILSON, D., WREN, S. \& REYNOLDS, G. 2012. Linking dissolution to disintegration in immediate release tablets using image analysis and a population balance modelling approach. Pharm Res, 29, 198-208.

WREN, S. A. C., ALHUSBAN, F., BARRY, A. R. \& HUGHES, L. P. 2017. Mechanistic understanding of the link between Sodium Starch Glycolate properties and the performance of tablets made by wet granulation. International Journal of Pharmaceutics, 529, 319-328.

WU, C.-Y., BEST, S. M., BENTHAM, A. C., HANCOCK, B. C. \& BONFIELD, W. 2006. Predicting the Tensile Strength of Compacted Multi-Component Mixtures of Pharmaceutical Powders. Pharmaceutical Research, 23, 1898-1905.

WU, S.-J. \& SUN, C. 2007. Insensitivity of Compaction Properties of Brittle Granules to Size Enlargement by Roller Compaction. Journal of Pharmaceutical Sciences, $96,1445-1450$.

ZEA, L. P., YUSOF, Y. A., AZIZ, M. G., LING, C. N. \& AMIN, N. A. M. 2013. Compressibility and dissolution characteristics of mixed fruit tablets made from guava and pitaya fruit powders. Powder Technology, 247, 112-119. 


\section{Appendix}

Appendix 1 Particle size distributions with time for Man100:MCC0 tablets produced using 20 bar RC pressure and $5 \mathrm{kN}$ tableting force.

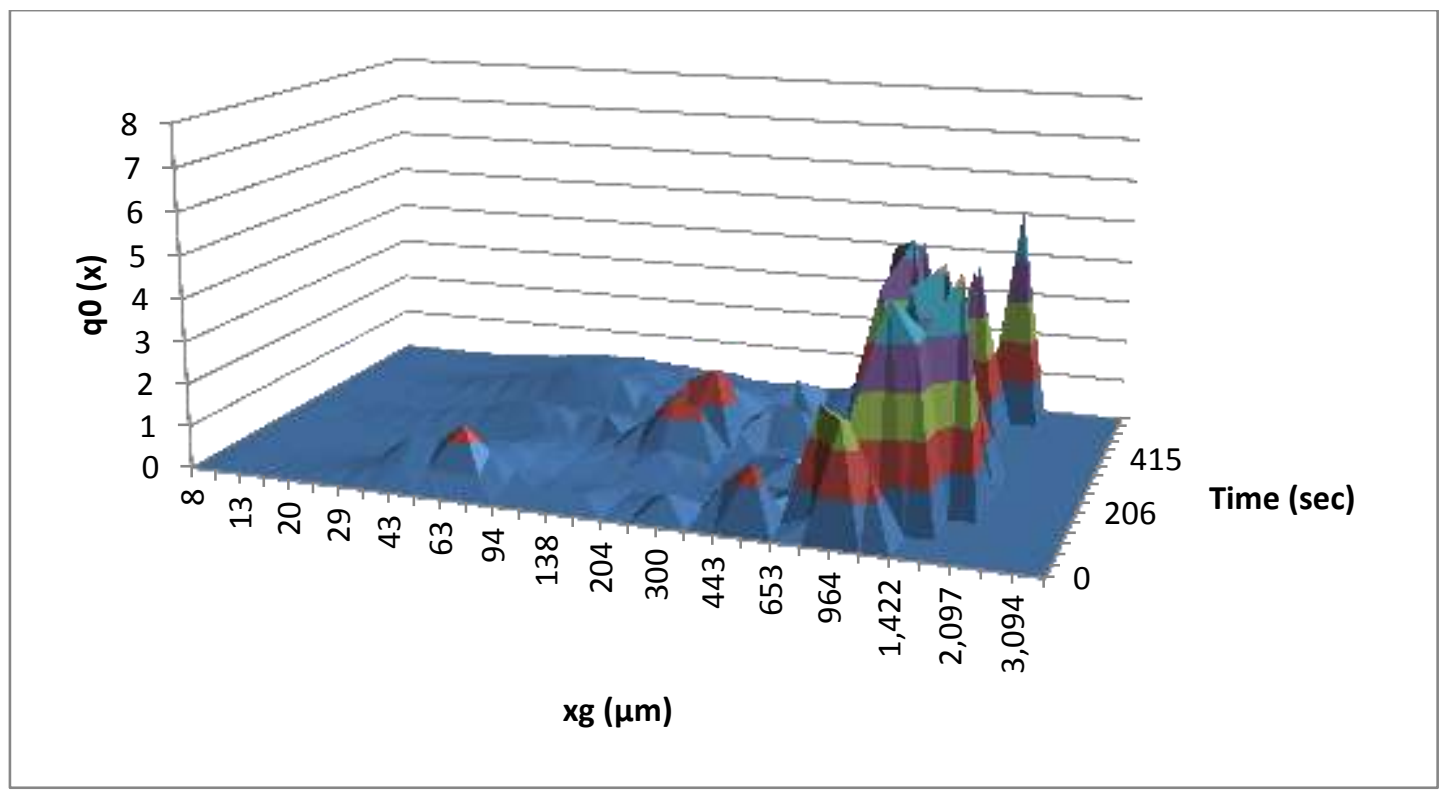

Appendix 2 Particle size distributions with time for Man100:MCC0 tablets produced using 100 bar RC pressure and $5 \mathrm{kN}$ tableting force.

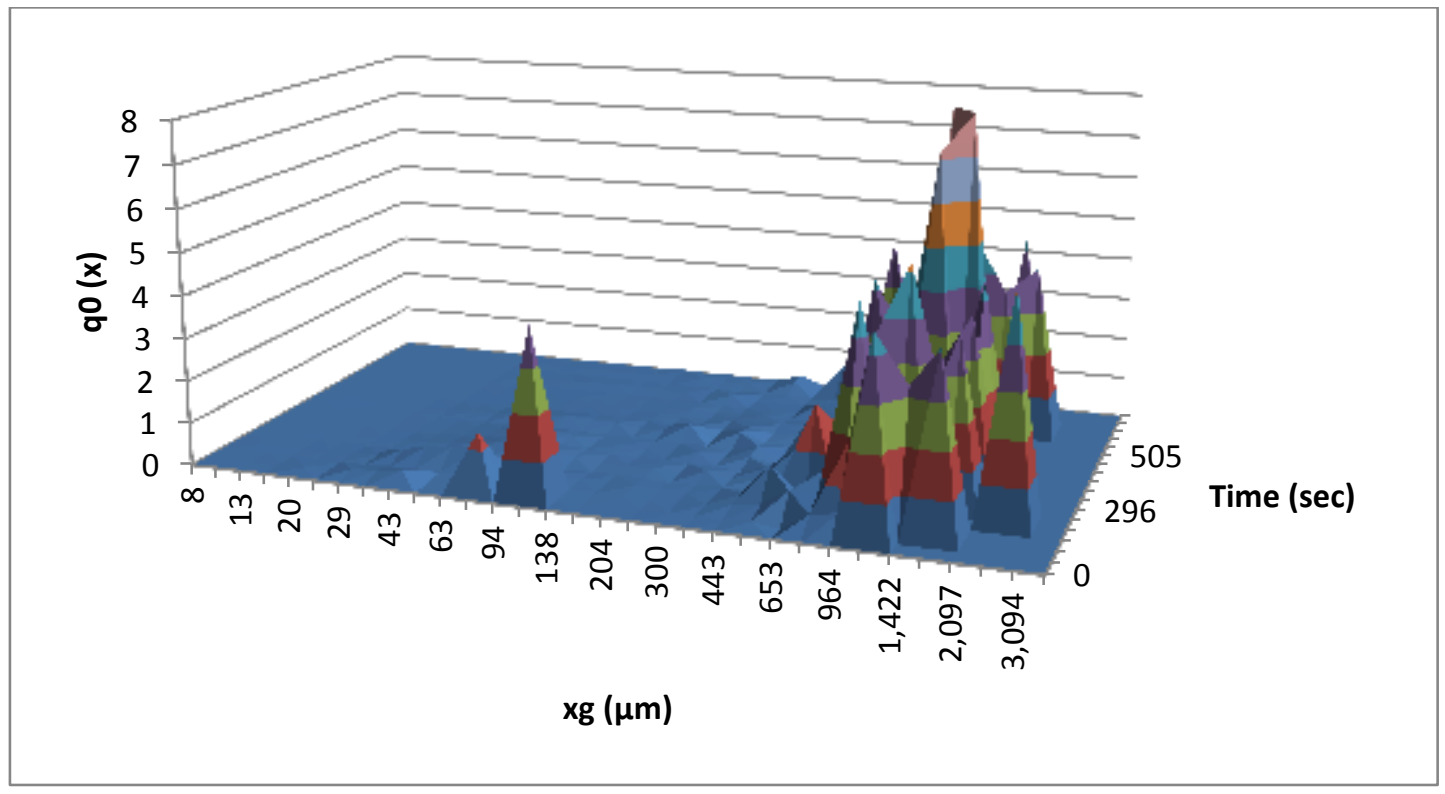


Appendix 3 Particle size distributions with time for Man100:MCC0 tablets produced using 20 bar RC pressure and $20 \mathrm{kN}$ tableting force.

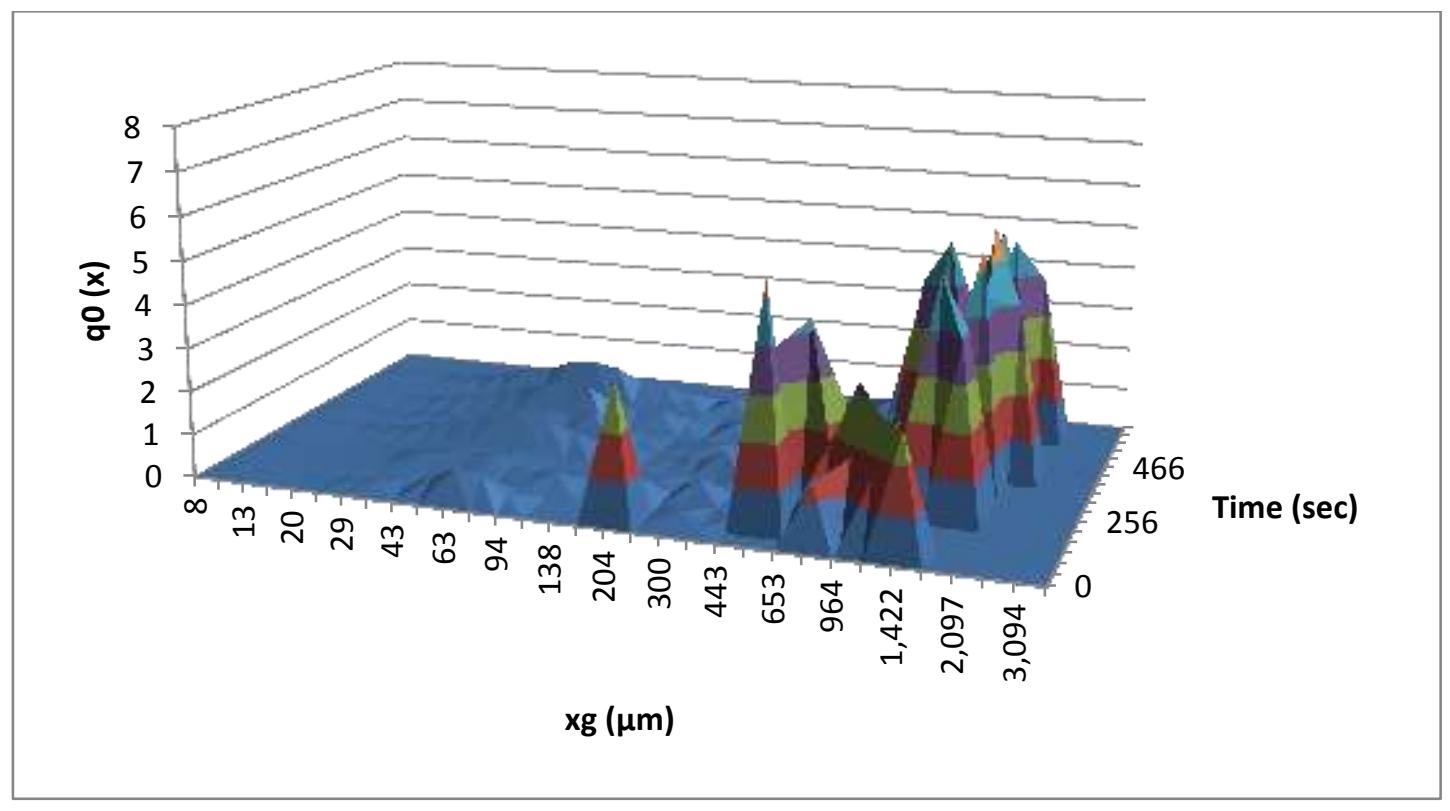

Appendix 4 Particle size distributions with time for Man100:MCC0 tablets produced using 100 bar RC pressure and $20 \mathrm{kN}$ tableting force.

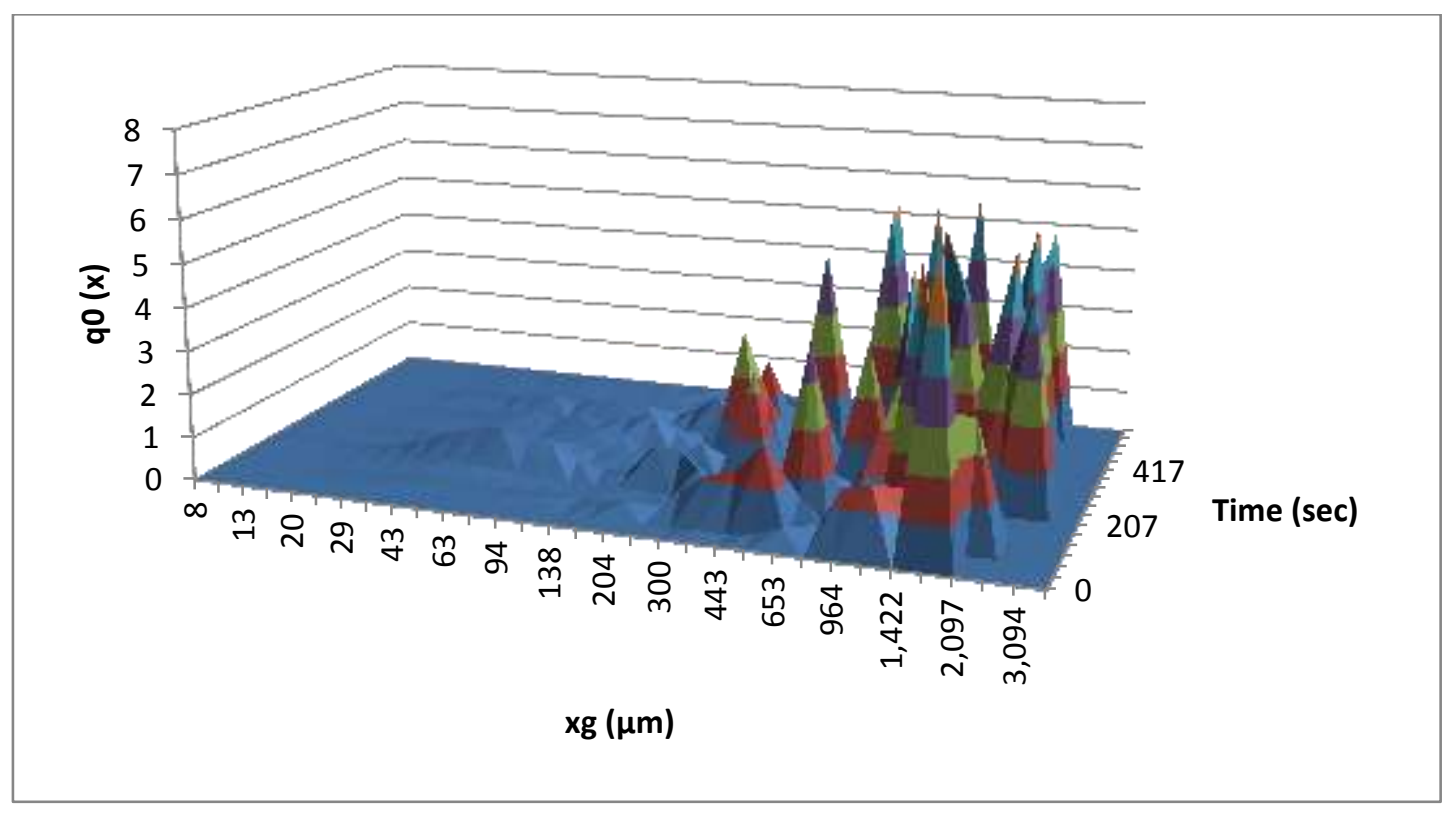


Appendix 5 Particle size distributions with time for Man75:MCC25 tablets produced using 20 bar RC pressure and $5 \mathrm{kN}$ tableting force.

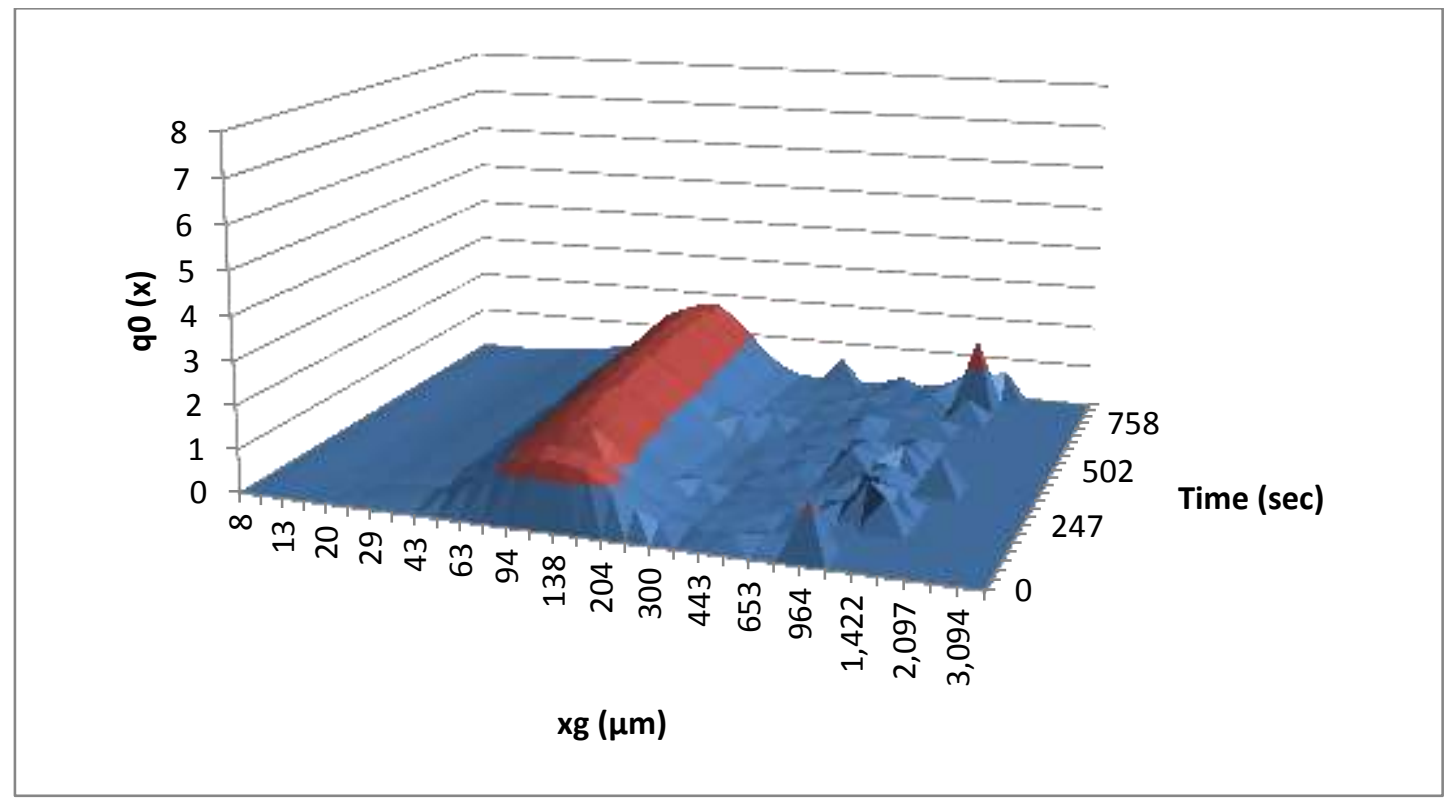

Appendix 6 Particle size distributions with time for Man75:MCC25 tablets produced using 100 bar RC pressure and $5 \mathrm{kN}$ tableting force.

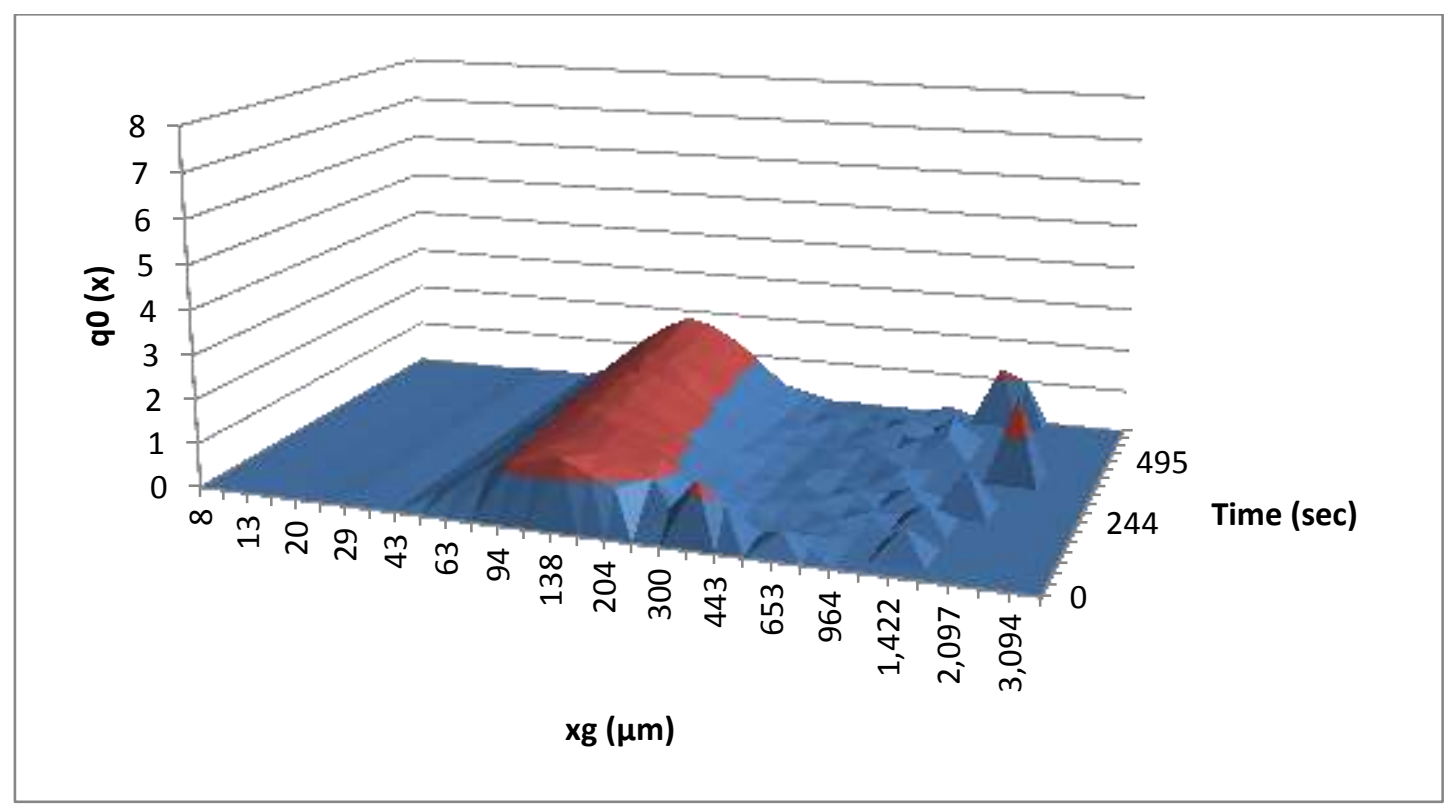


Appendix 7 Particle size distributions with time for Man75:MCC25 tablets produced using 20 bar RC pressure and $20 \mathrm{kN}$ tableting force.

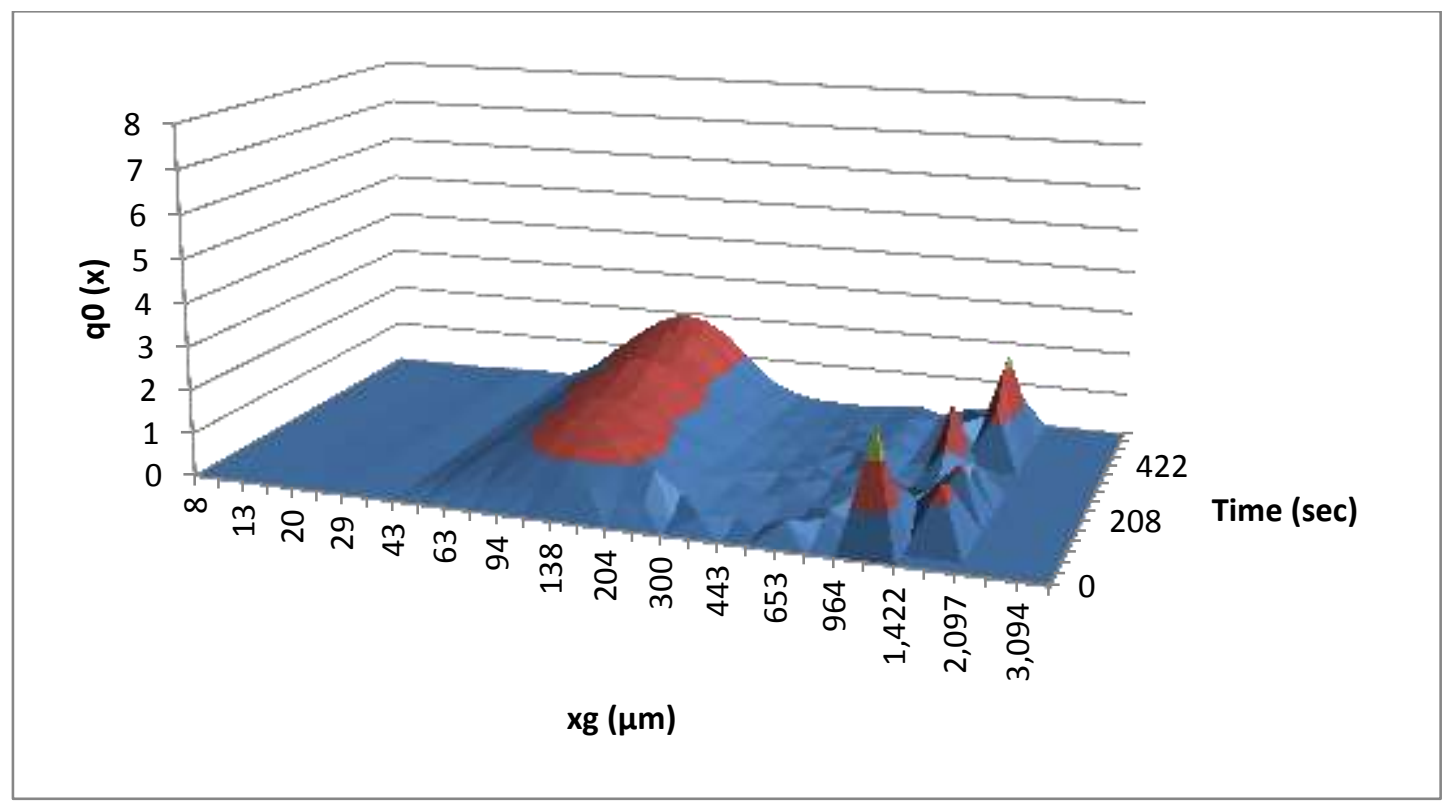

Appendix 8 Particle size distributions with time for Man75:MCC25 tablets produced using 100 bar RC pressure and $20 \mathrm{kN}$ tableting force.

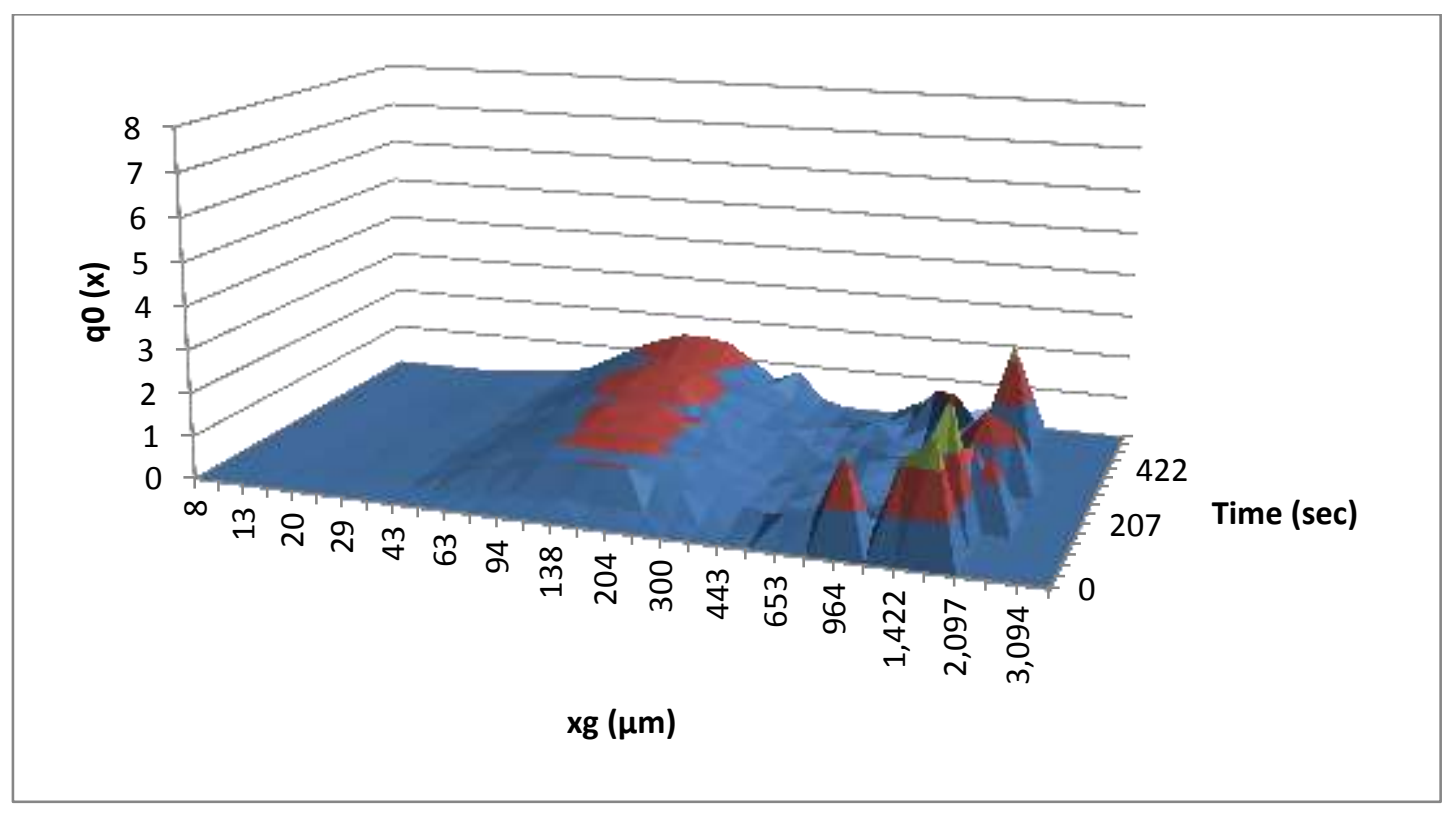


Appendix 9 Particle size distributions with time for Man25:MCC75 tablets produced using 20 bar RC pressure and $5 \mathrm{kN}$ tableting force.

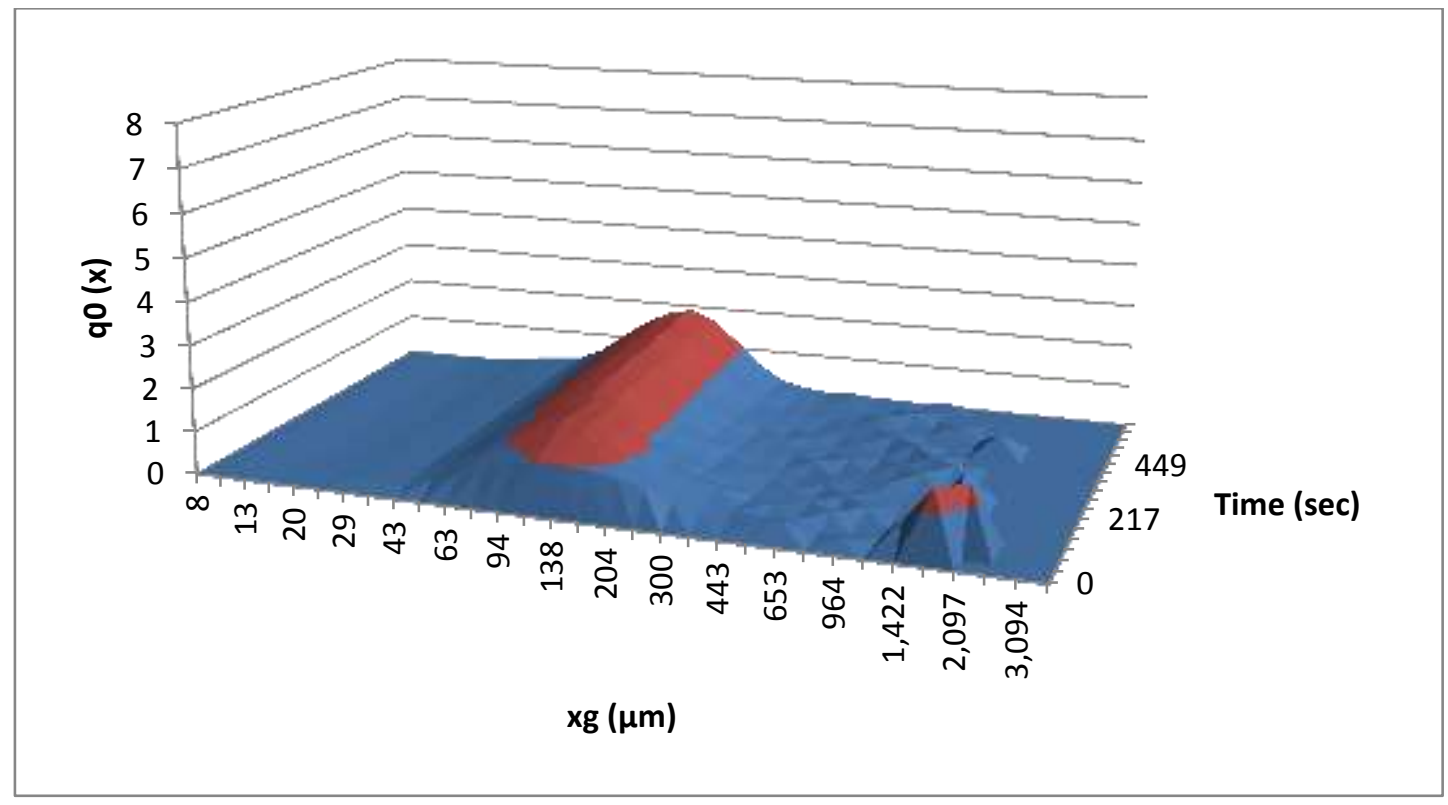

Appendix 10 Particle size distributions with time for Man25:MCC75 tablets produced using 100 bar RC pressure and $5 \mathrm{kN}$ tableting force.

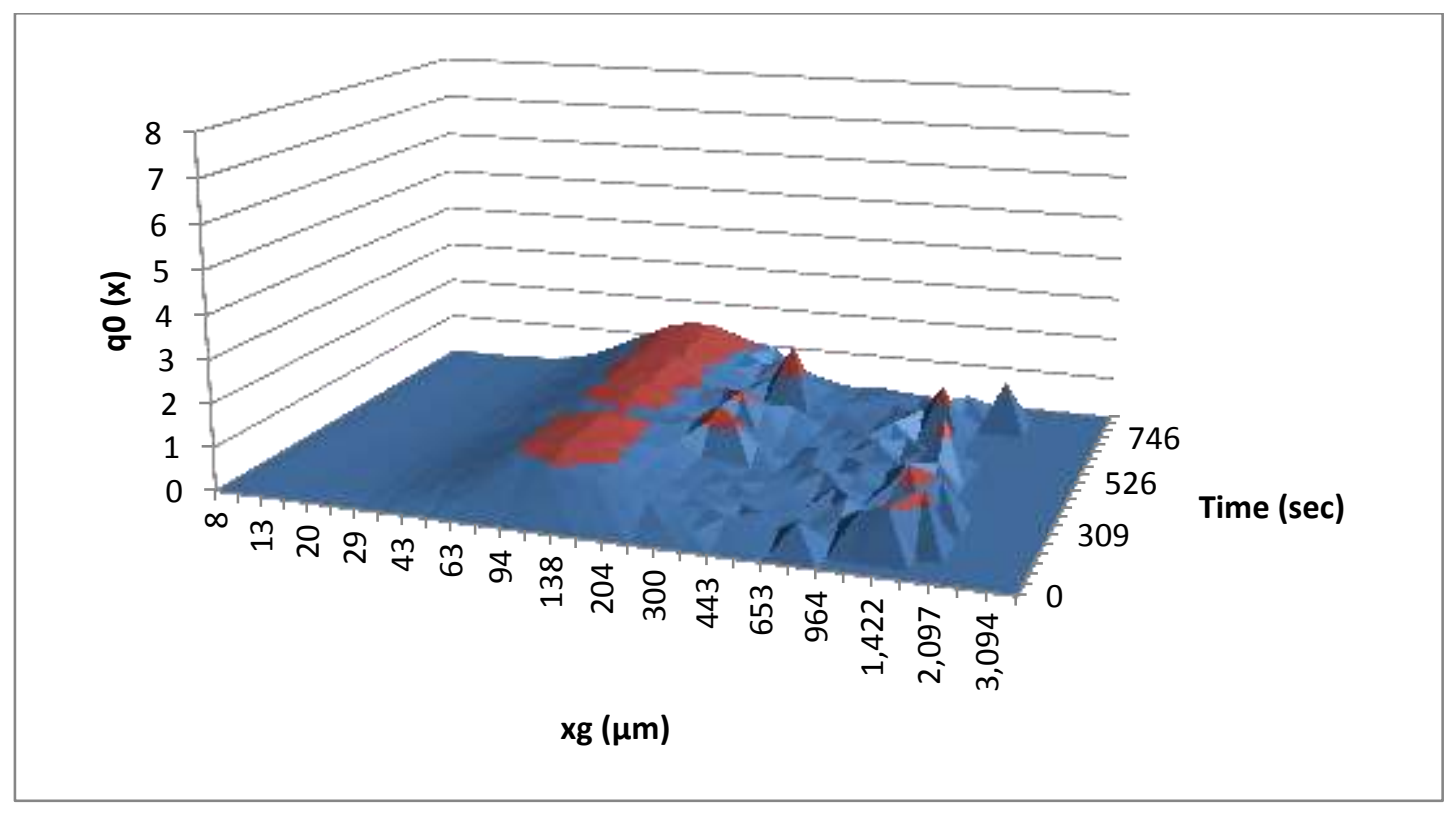


Appendix 11 Particle size distributions with time for Man25:MCC75 tablets produced using 20 bar RC pressure and $20 \mathrm{kN}$ tableting force.

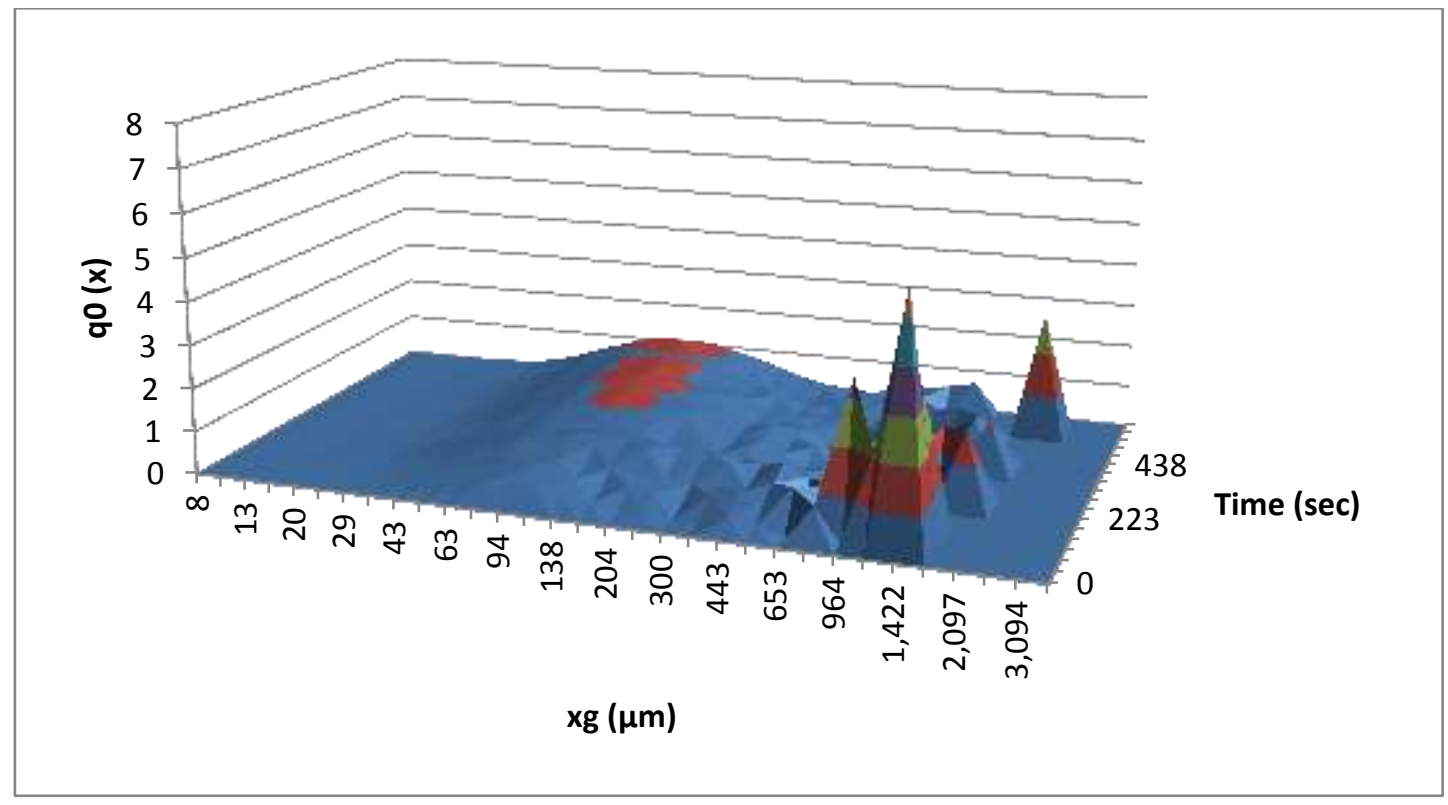

Appendix 12 Particle size distributions with time for Man25:MCC75 tablets produced using 100 bar RC pressure and $20 \mathrm{kN}$ tableting force.

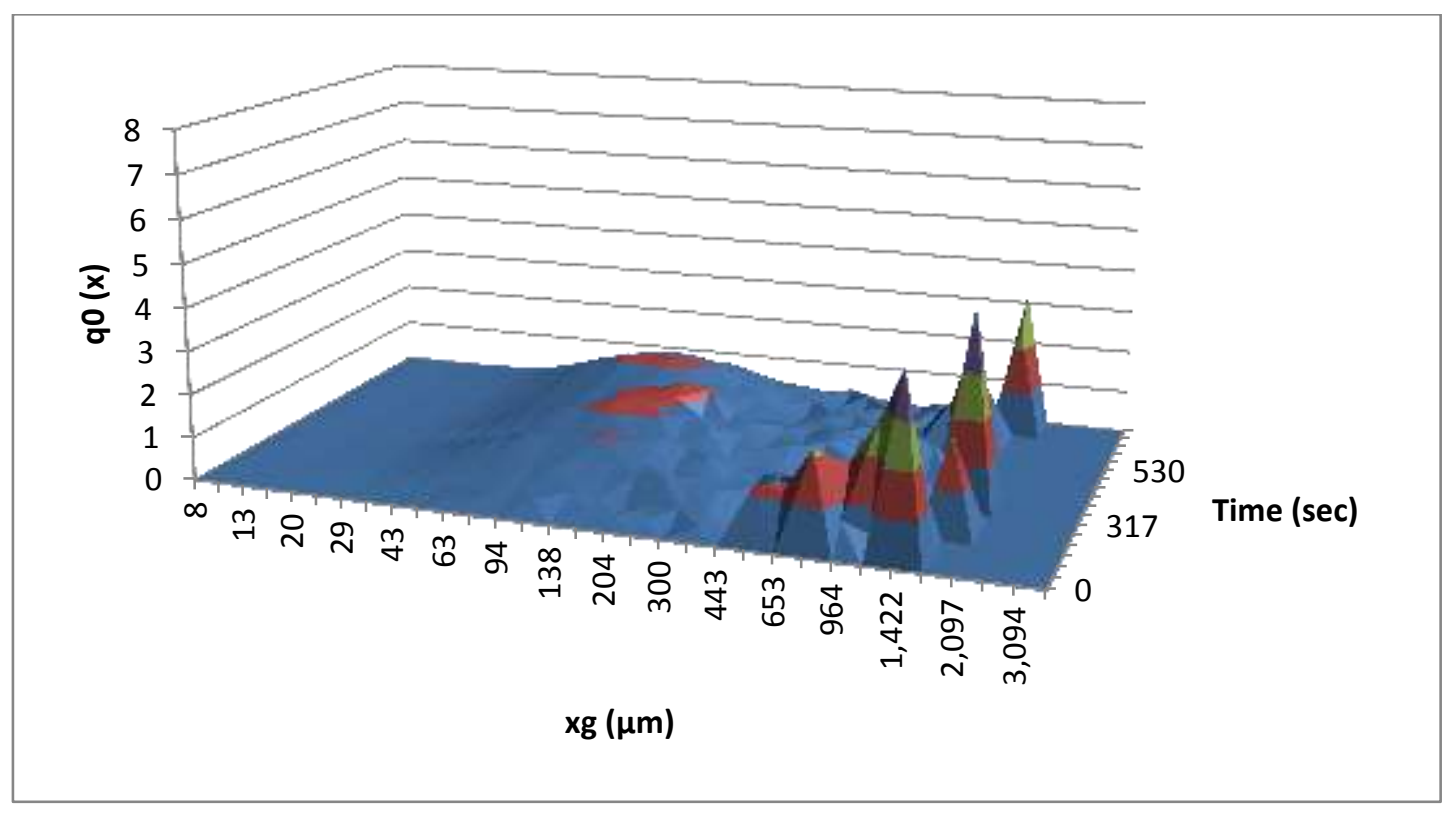


Appendix 13 Particle size distributions with time for Man0:MCC10 tablets produced using 20 bar RC pressure and $5 \mathrm{kN}$ tableting force.

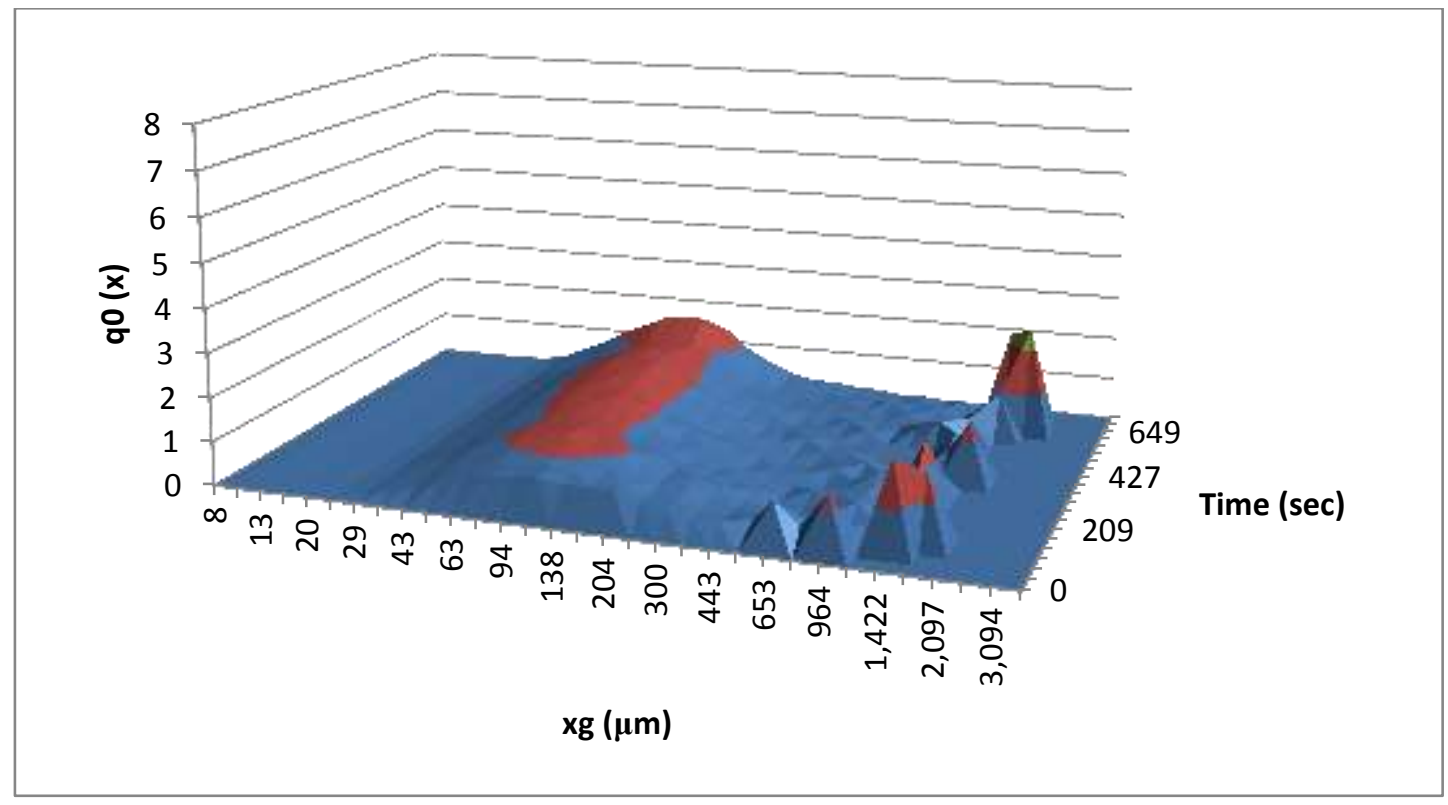

Appendix 14 Particle size distributions with time for Man0:MCC10 tablets produced using 100 bar RC pressure and $5 \mathrm{kN}$ tableting force.

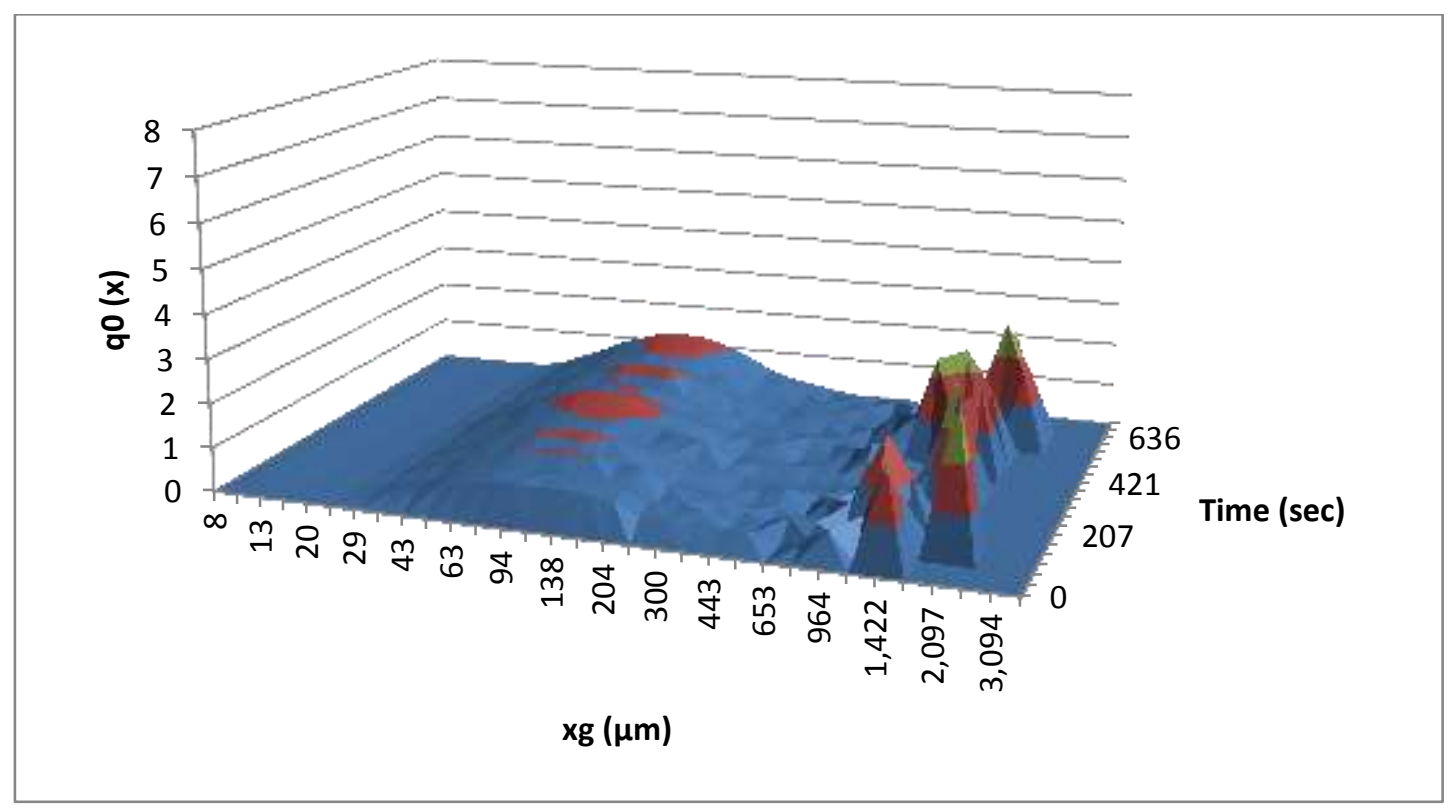


Appendix 15 Particle size distributions with time for Man0:MCC10 tablets produced using 20 bar RC pressure and $20 \mathrm{kN}$ tableting force.

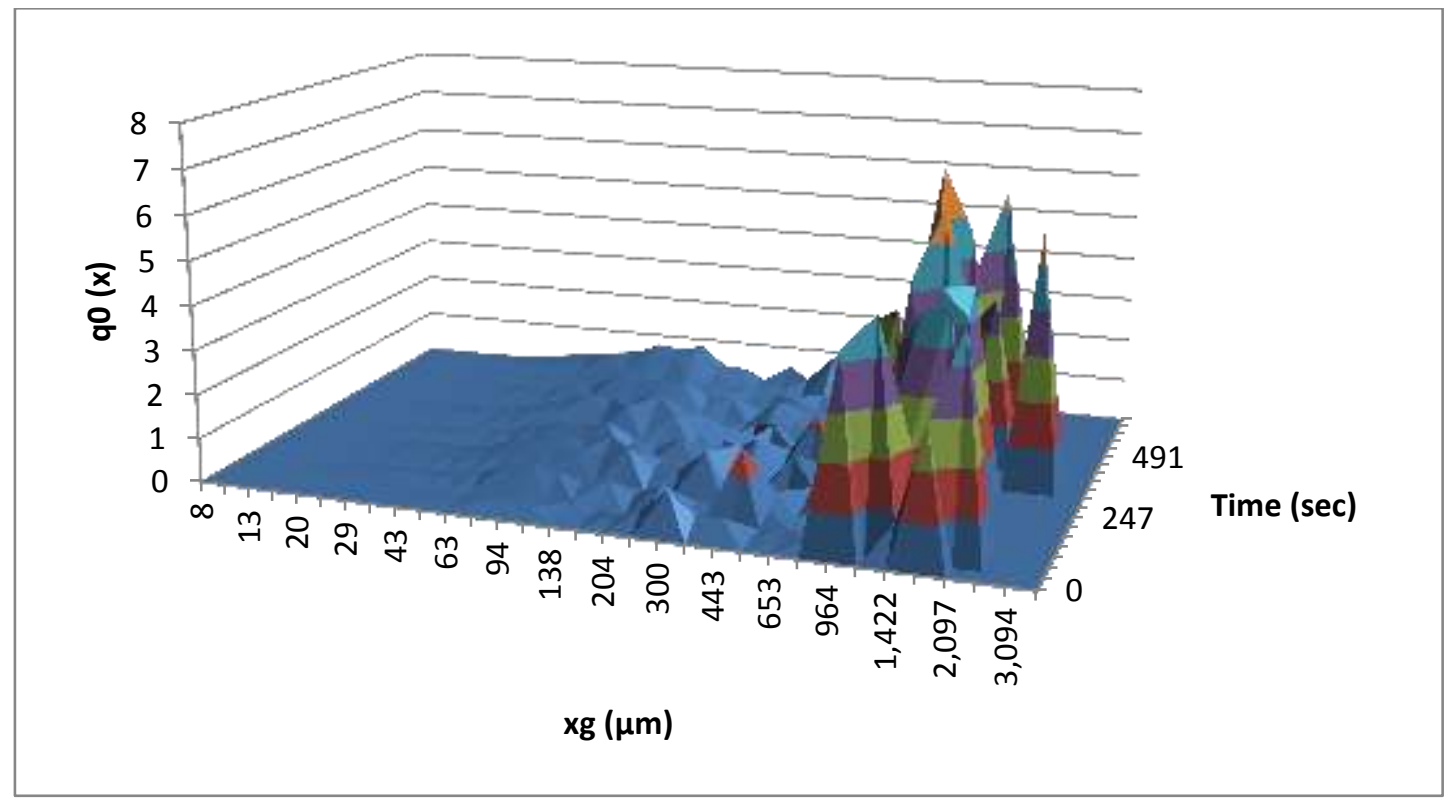

Appendix 16 Particle size distributions with time for Man0:MCC10 tablets produced using 100 bar RC pressure and $20 \mathrm{kN}$ tableting force.

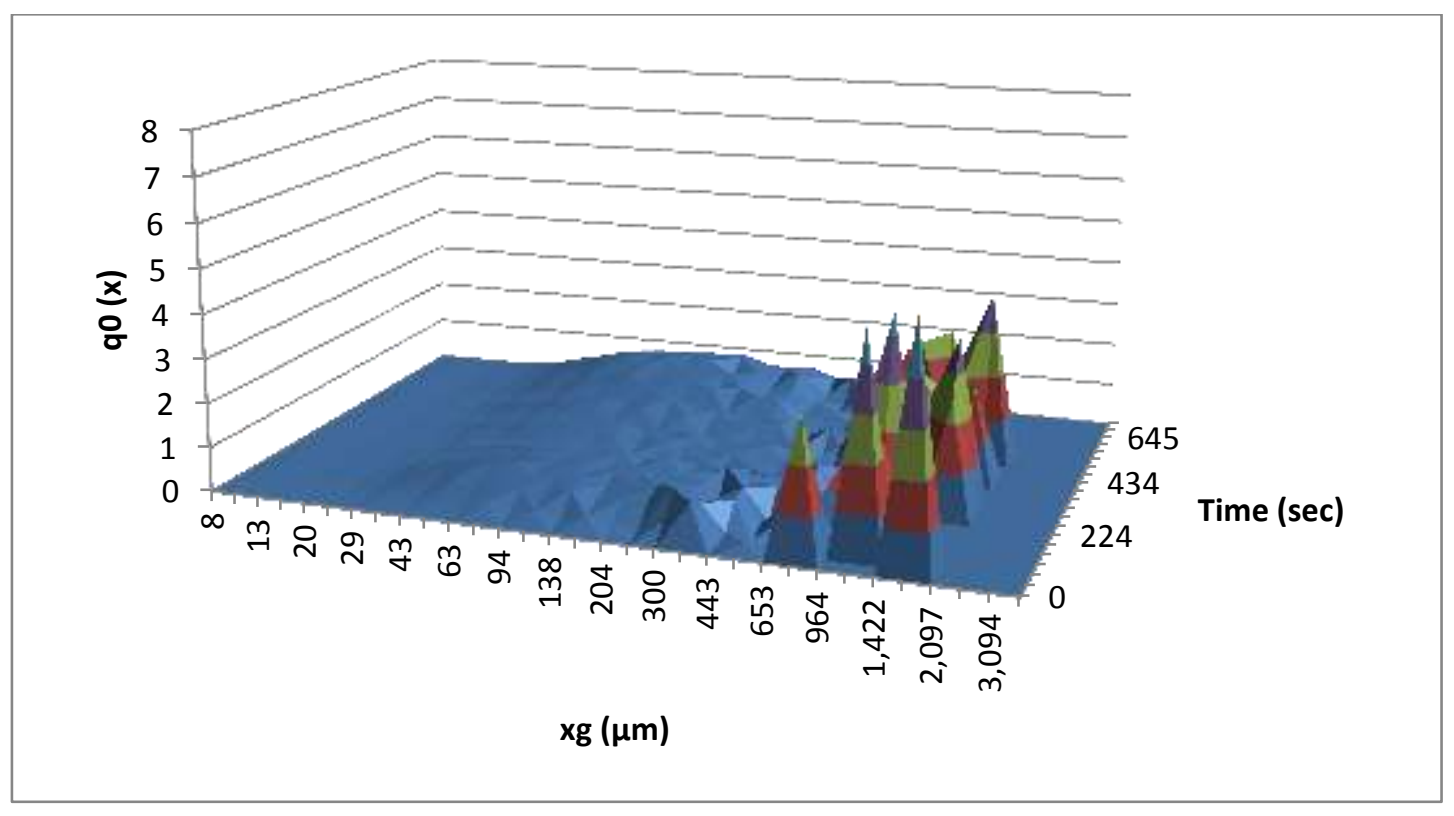


Appendix 17 Indication of the volume of solid in solution during the LIXELL measurement, determined using the $D_{50}$ values and the number of particles released.
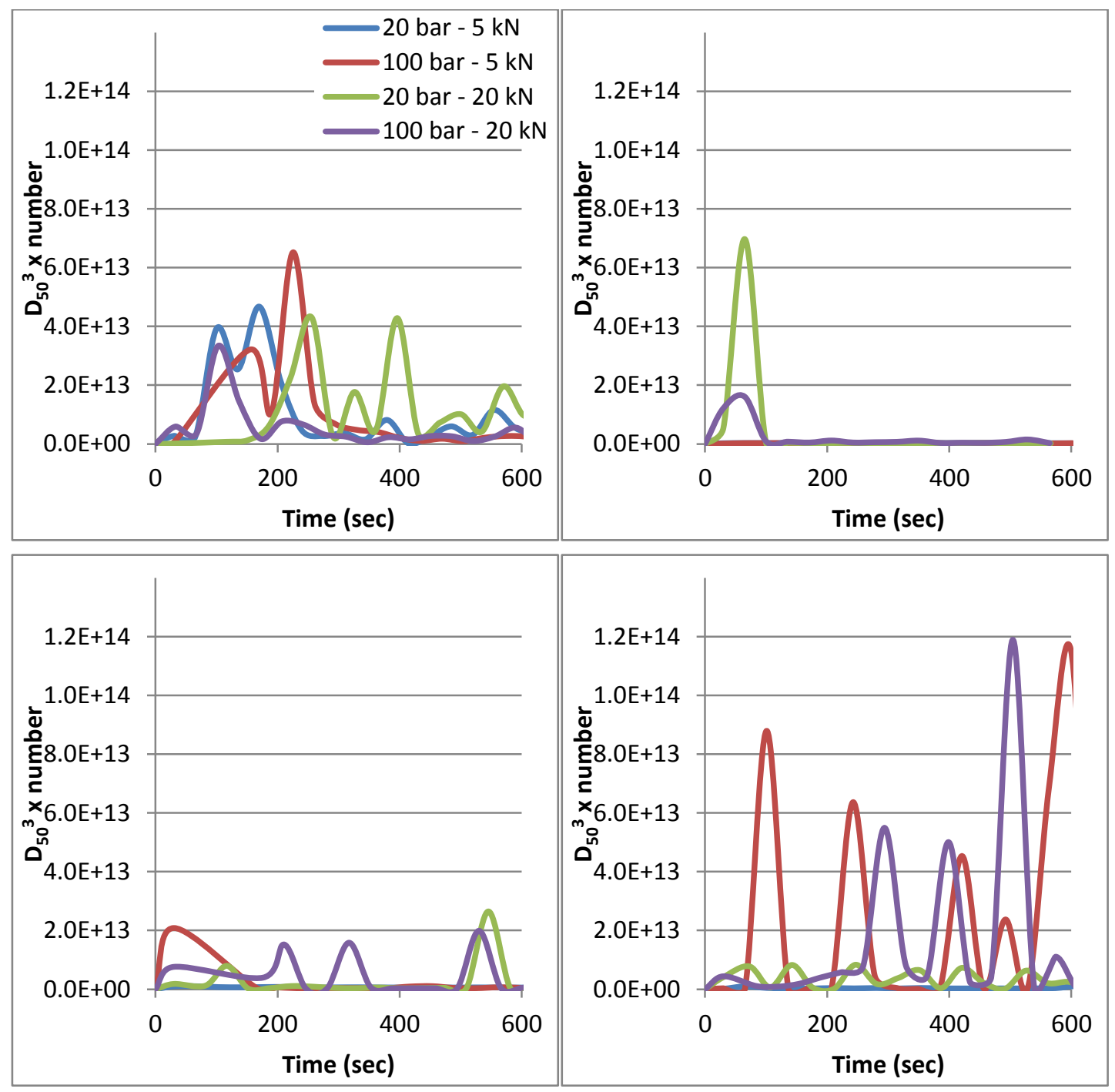
Appendix 18 MATLAB code developed by Xavier Mesnier, adapted for the project. (Mesnier et al., 2013)

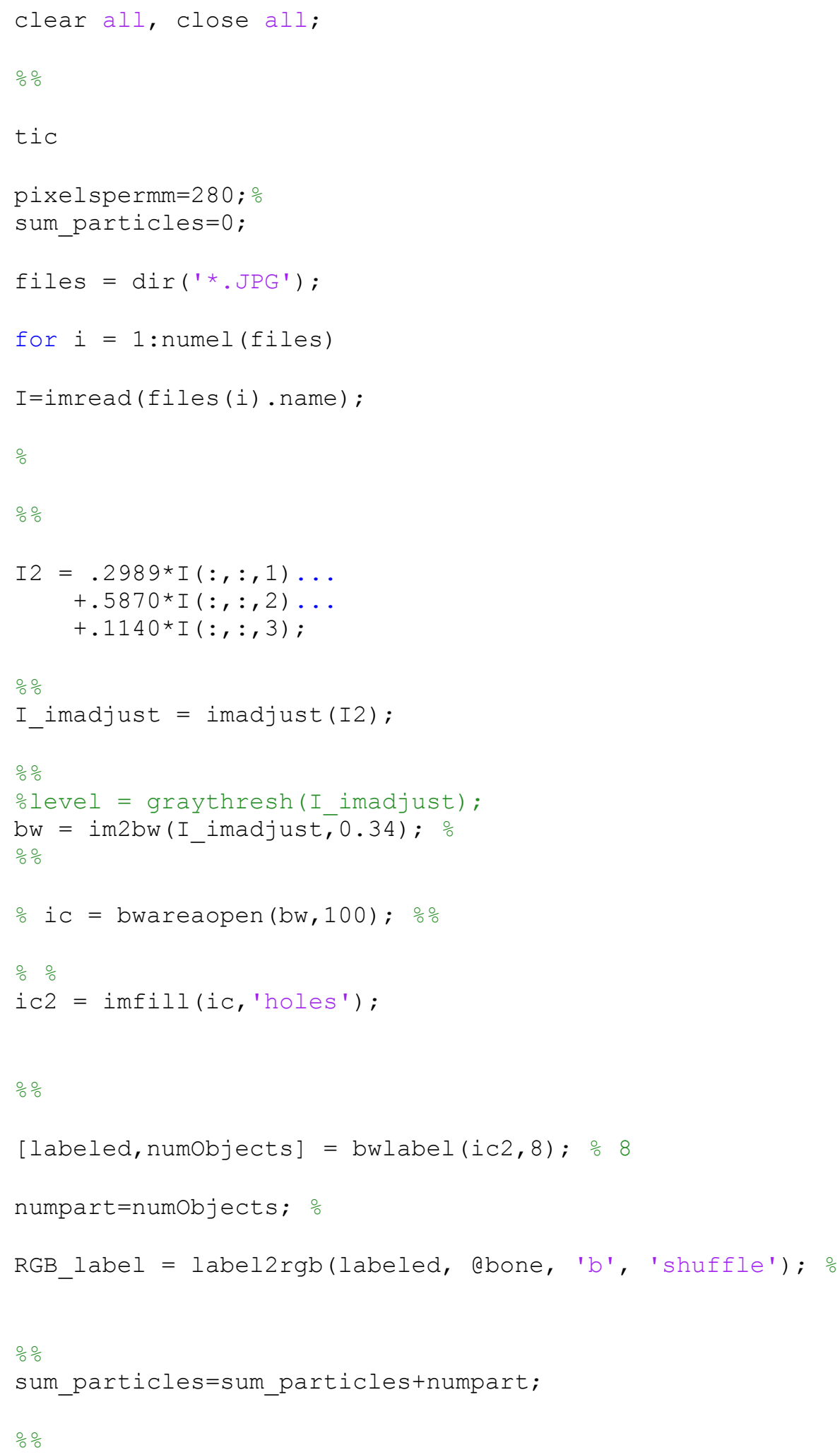




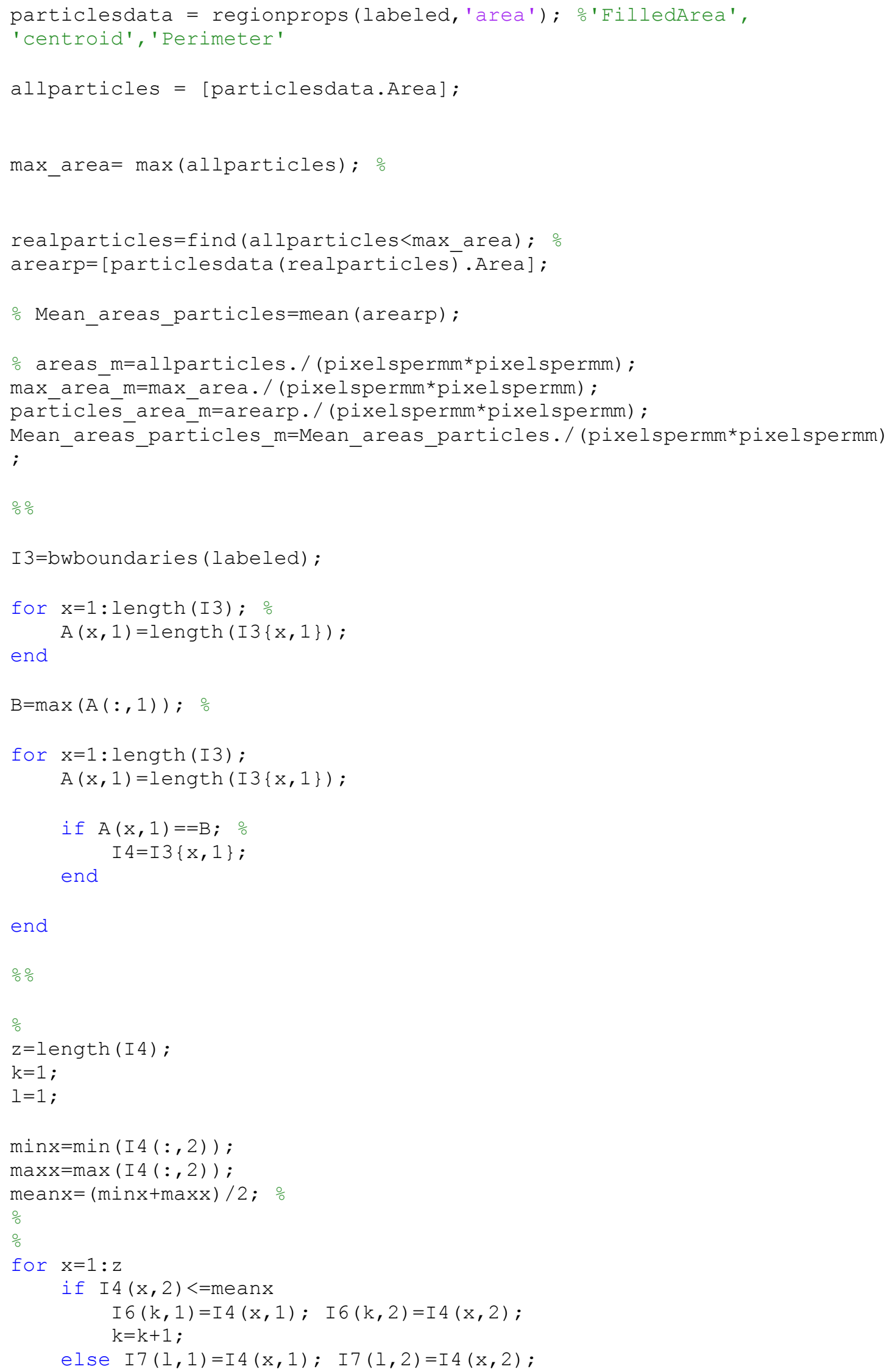




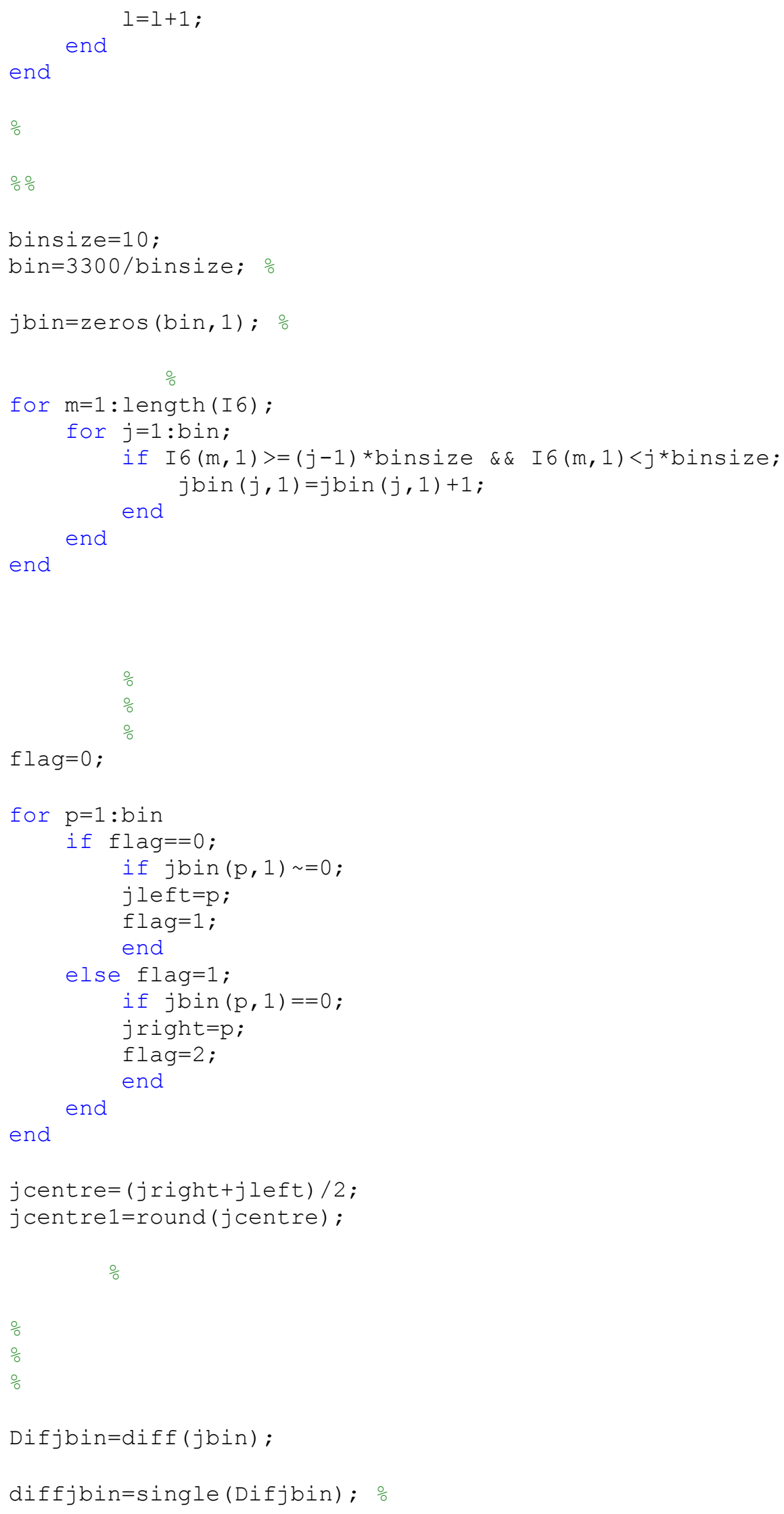




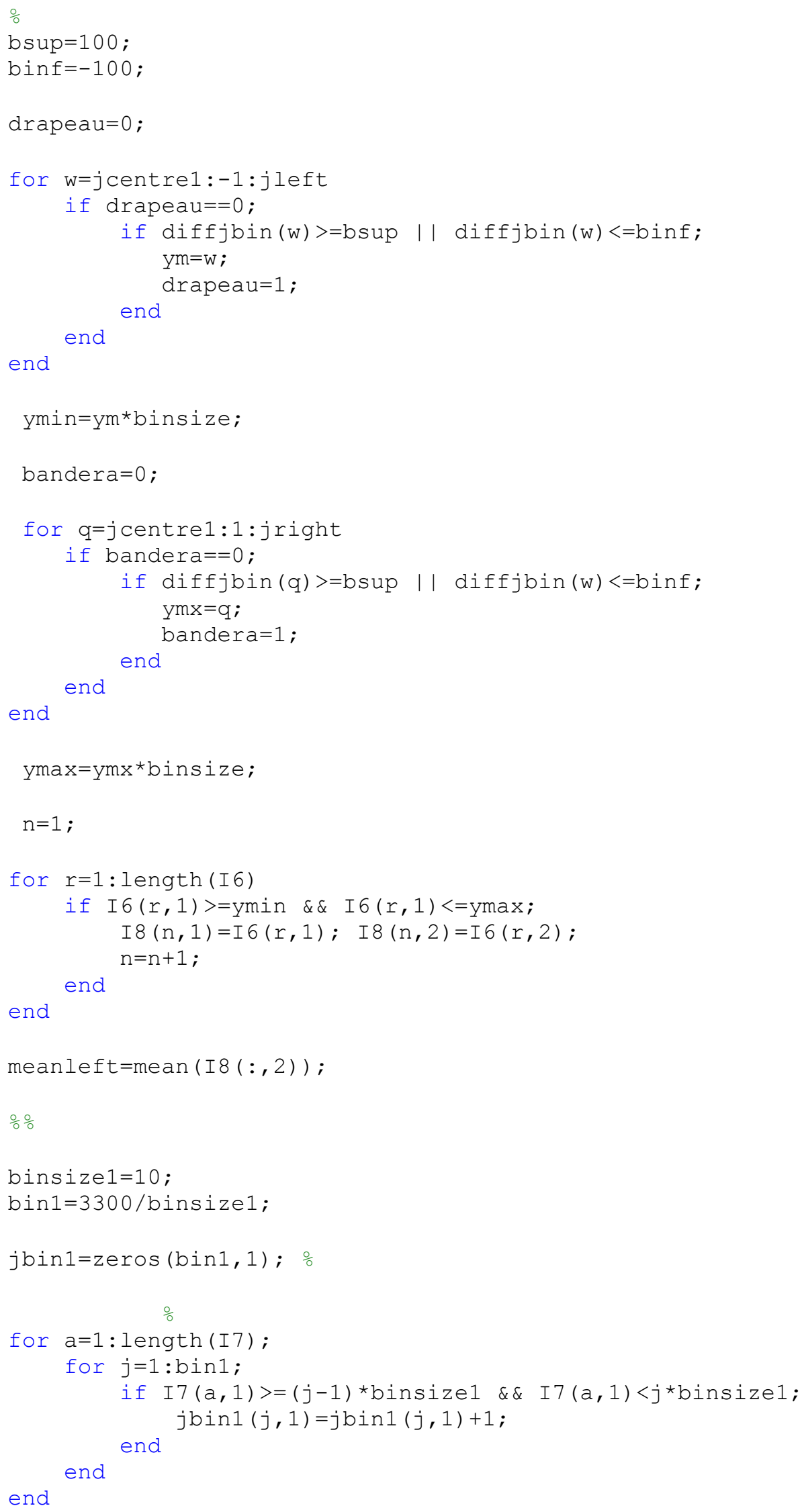




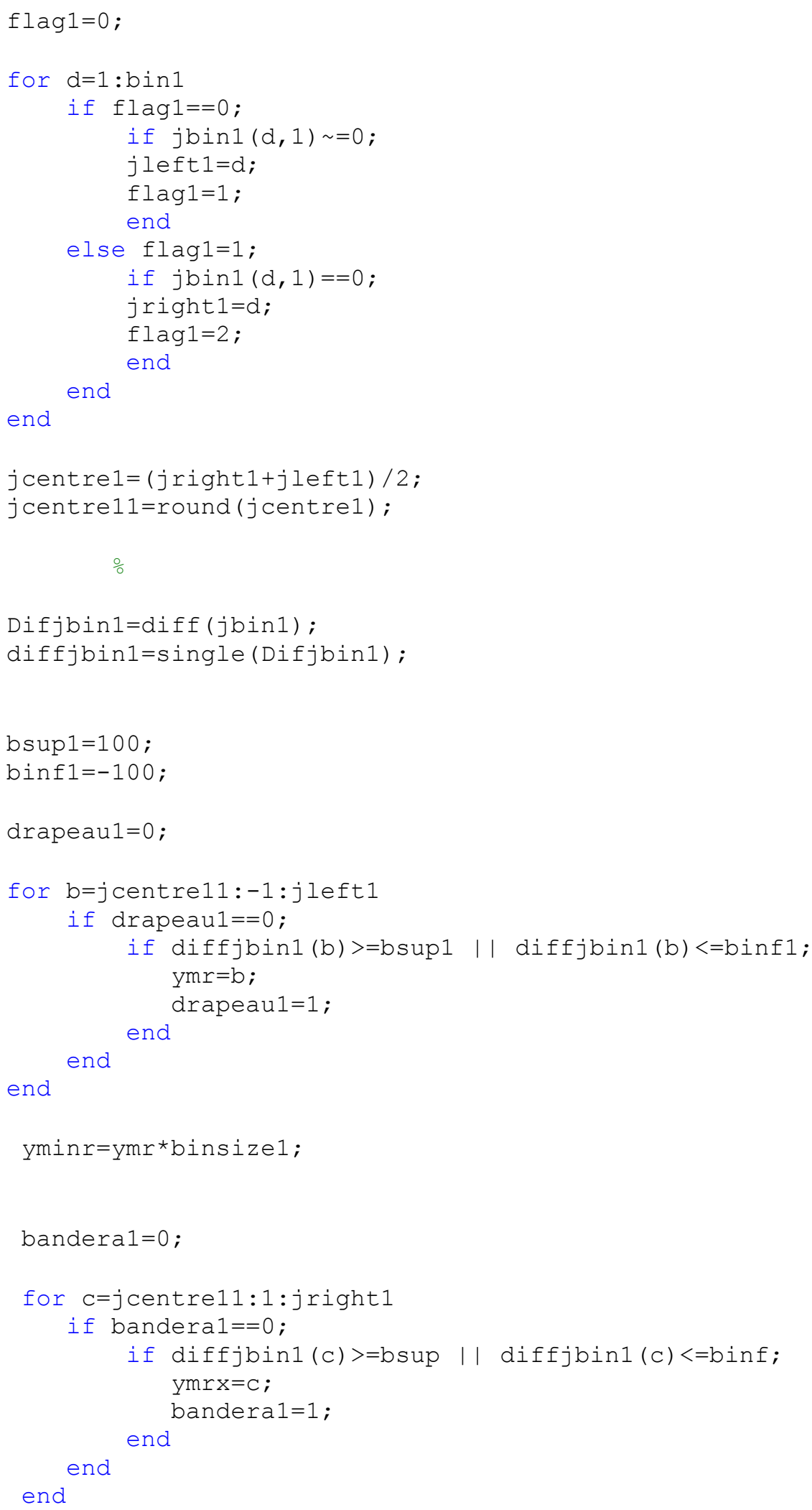




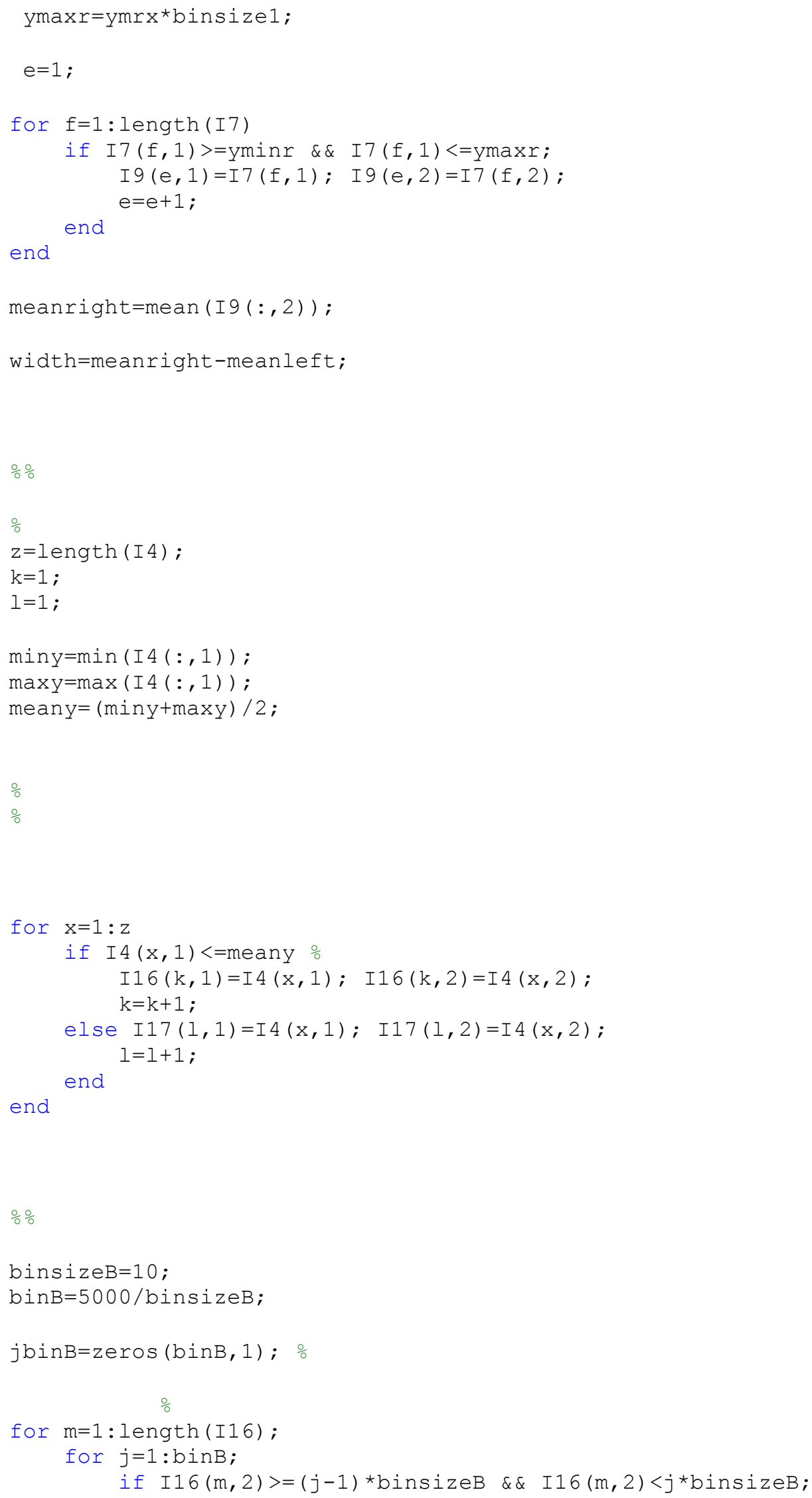




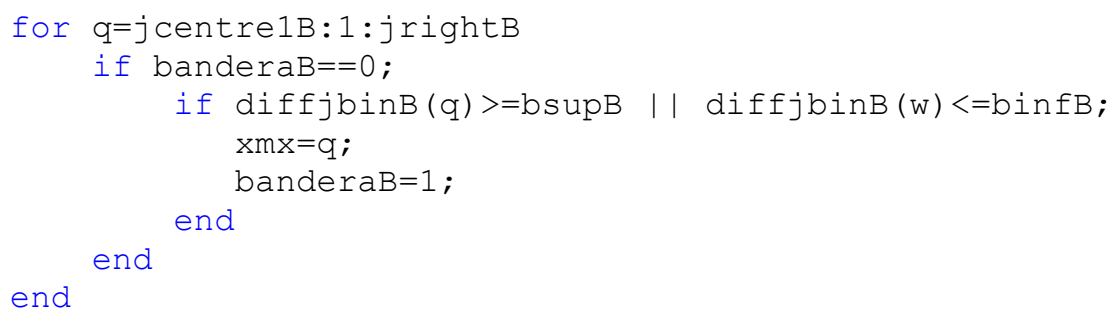




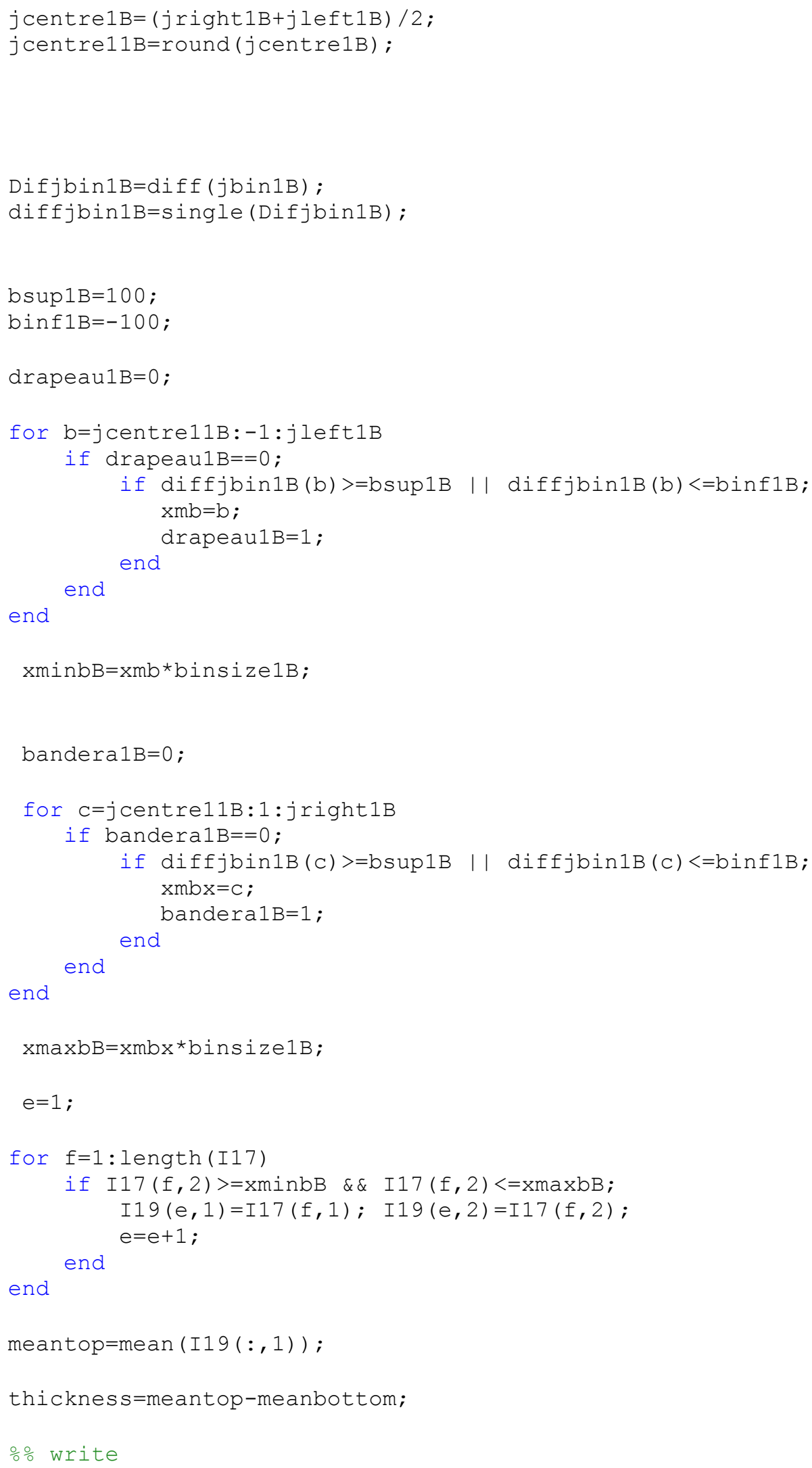


widthmm=width/pixelspermm;

thicknessmm=thickness/pixelspermm;

dlmwrite('output_area.csv', areas_m, '-append', 'newline', 'pc') ;

dlmwrite('output_maxarea.csv', max_area_m, '-append', 'newline', 'pc')

dlmwrite('output_partarea.csv',particles_area_m , '-

append', 'newline', 'pc')

dlmwrite('output_numparticles.csv', numpart, '-append', 'newline', 'pc')

dlmwrite('output_sumparticle.csv', sum_particles, 'append', 'newline', 'pc')

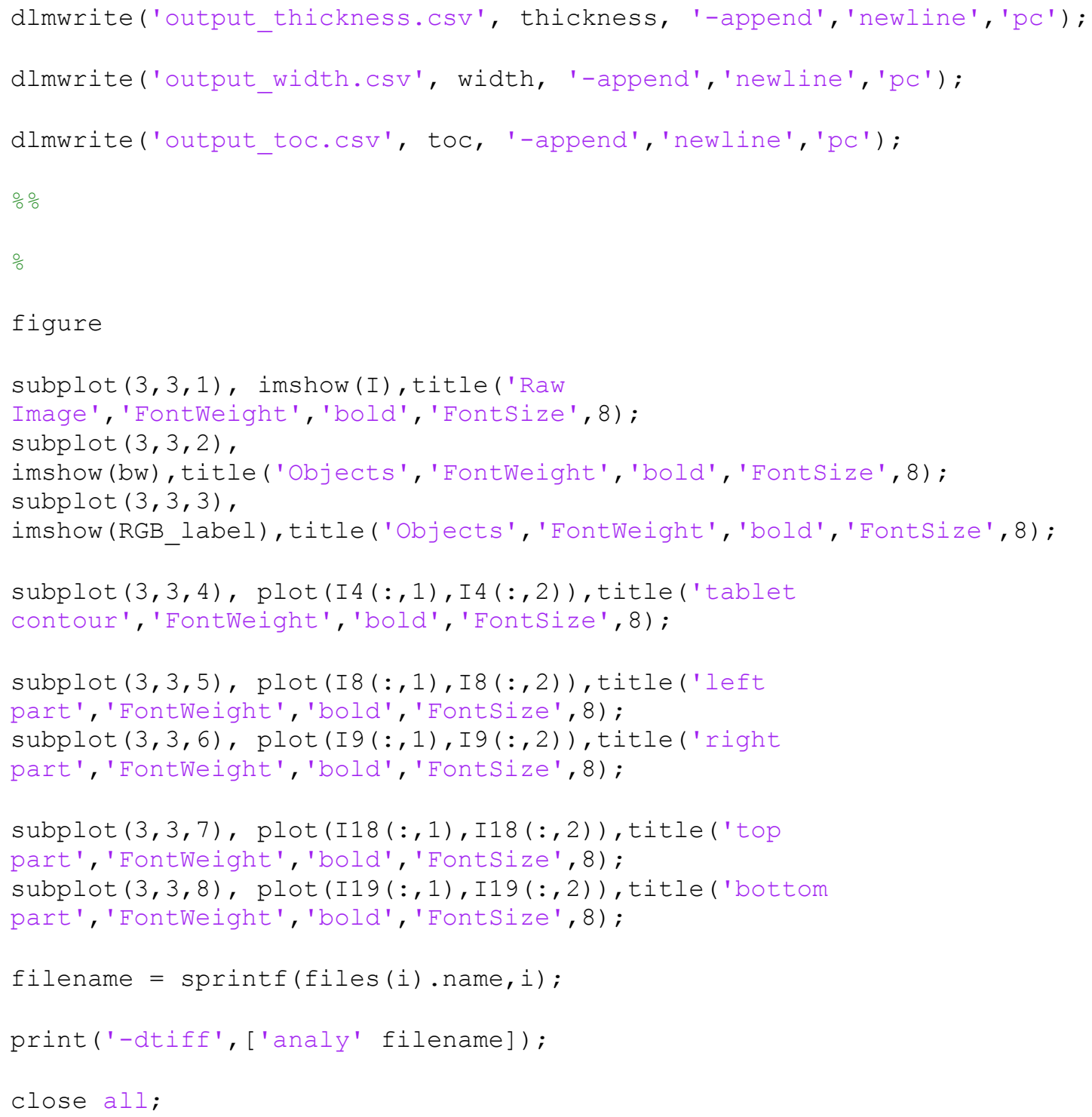


toc

end

Appendix 19 Image obtained during the Flow Cell analysis for Man0:MCC100 tablets produced using 20 bar RC pressure and $5 \mathrm{kN}$ tableting force.

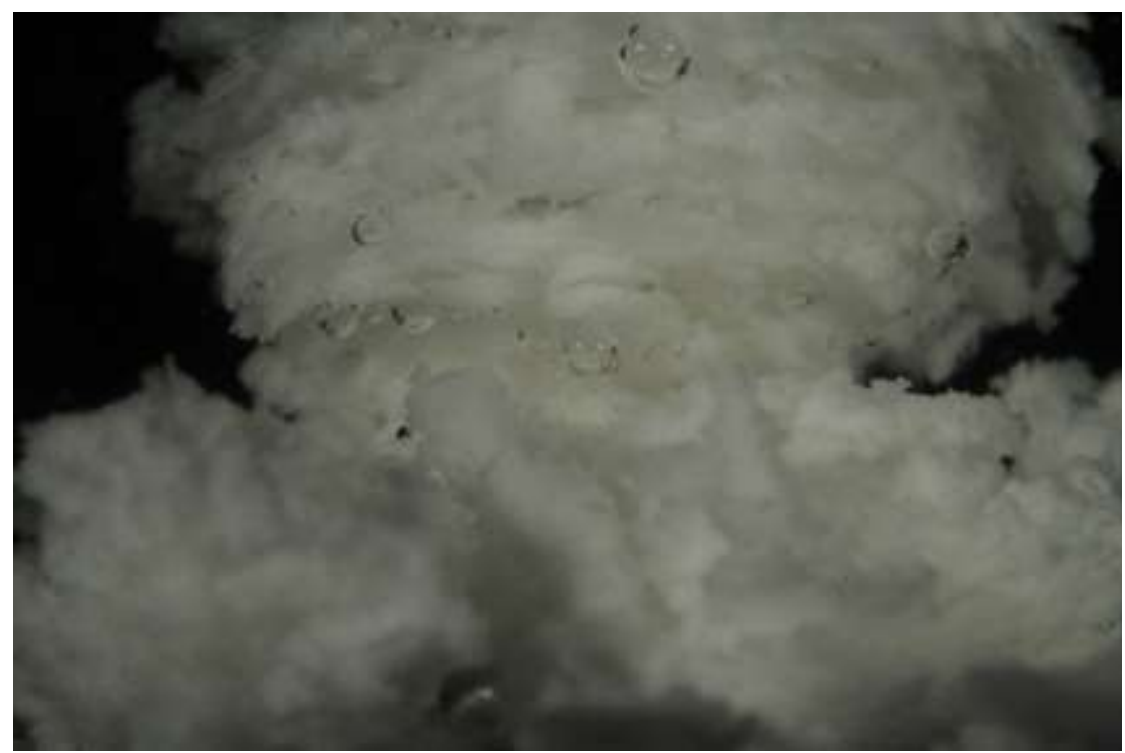




\section{Appendix 20 MATLAB code developed by Kimiaki Washino and Daniel Stevenson to determine the particle size distribution of the released particles during Flow Cell analysis.}

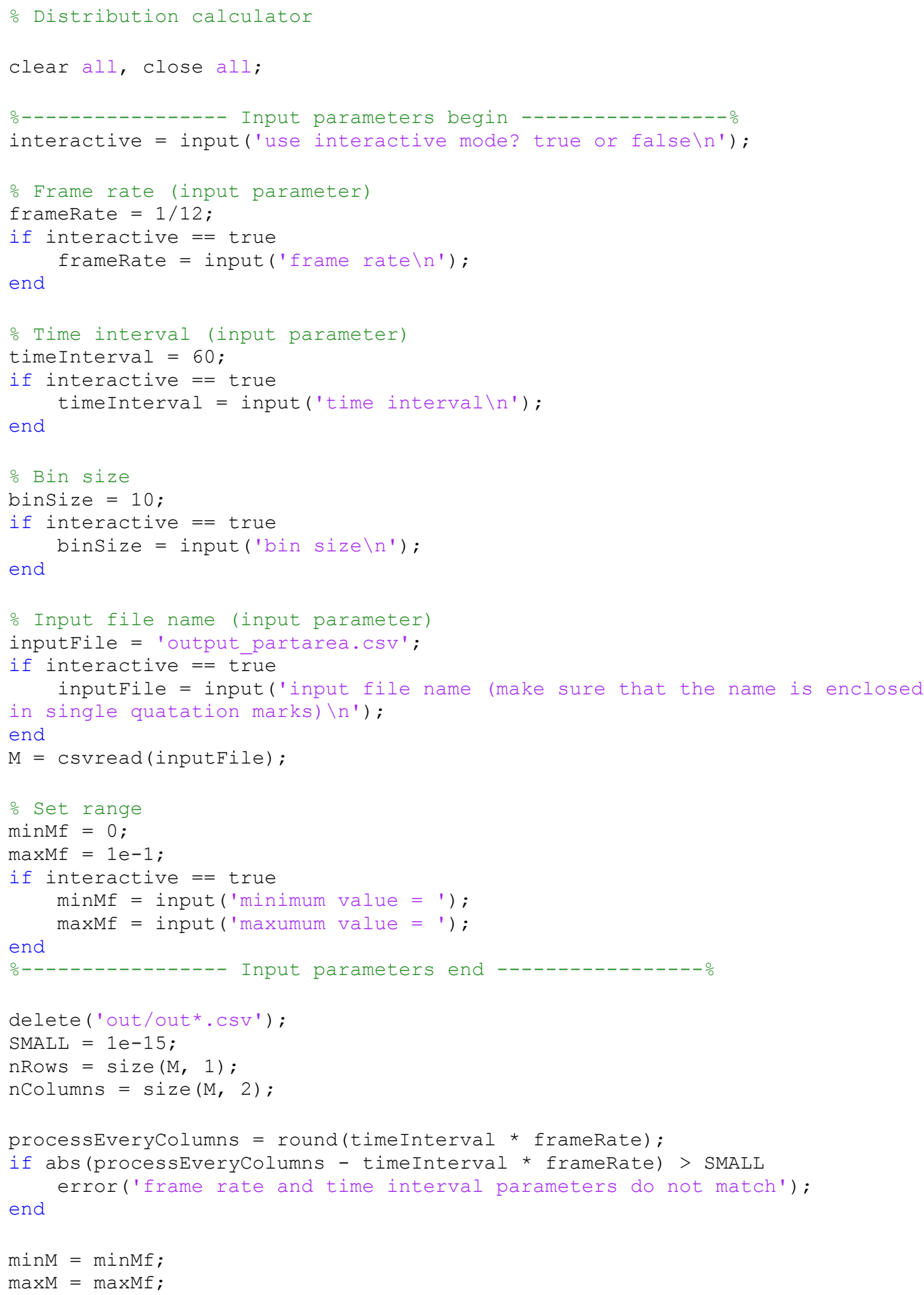




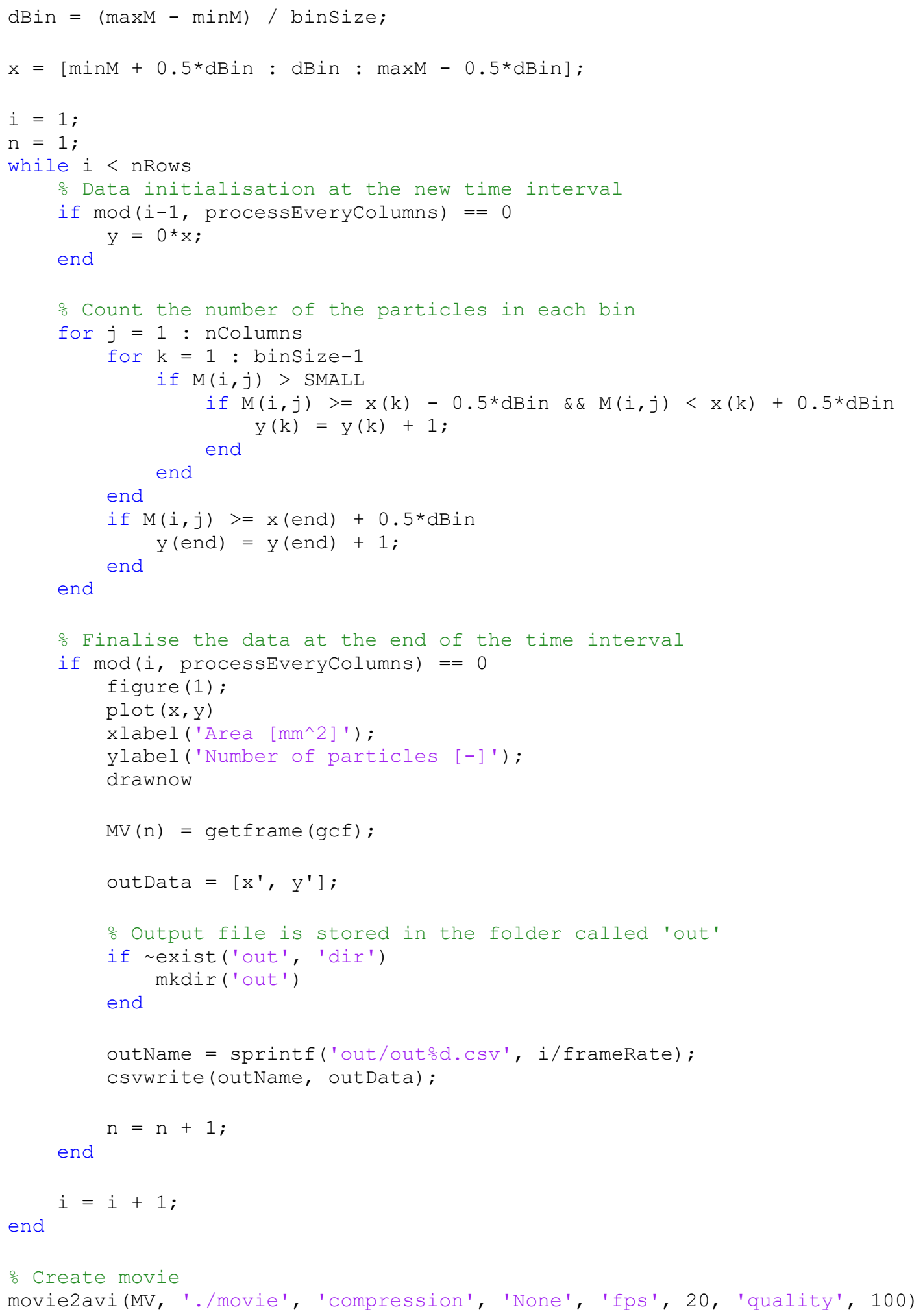

